\title{
A STUDY OF \\ THROMBOGENESIS IN ATRIAL FIBRILLATION \\ AND LEFT VENTRICULAR DYSFUNCTION
}

Thesis submitted to the University of Glasgow

for the Degree of

DOCTOR OF MEDICINE (M.D.)

BY

GREGORY - YOKE HONG LIP

1993

Department of Cardiology

Stobhill Hospital

Glasgow G21 3UW 
ProQuest Number: 13818543

All rights reserved

INFORMATION TO ALL USERS

The quality of this reproduction is dependent upon the quality of the copy submitted.

In the unlikely event that the author did not send a complete manuscript and there are missing pages, these will be noted. Also, if material had to be removed, a note will indicate the deletion.

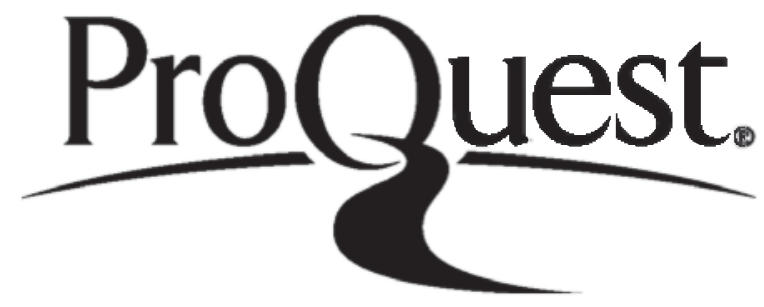

ProQuest 13818543

Published by ProQuest LLC (2018). Copyright of the Dissertation is held by the Author.

All rights reserved.

This work is protected against unauthorized copying under Title 17, United States Code Microform Edition (C) ProQuest LLC.

ProQuest LLC.

789 East Eisenhower Parkway

P.O. Box 1346

Ann Arbor, Ml $48106-1346$ 


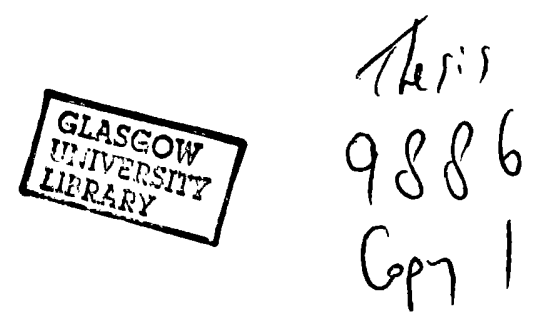




\section{TABLE OF CONTENTS}

PAGE NO

TITLE

CONTENTS 2

LIST OF TABLES 5

$\begin{array}{ll}\text { LIST OF FIGURES } & 7\end{array}$

$\begin{array}{lr}\text { DECLARATION } & 9\end{array}$

$\begin{array}{ll}\text { ACKNOWLEDGEMENTS } & 10\end{array}$

$\begin{array}{ll}\text { SUMMARY } & 11\end{array}$

LAYOUT OF THESIS 16

$\begin{array}{lll}\text { CHAPTER } 1 & 1 \text { INTRODUCTION } & 18\end{array}$

1.1 Thrombogenesis and prothrombotic factors

1.2 Fibrinogen, fibrin D-dimer, von Willebrand factor and lipoprotein(a)

in cardiovascular disease: A review of the literature

1.3 Aims of studies reported in this thesis

CHAPTER 2 A SURVEY OF ATRIAL FIBRILLATION 56

2.1 An overview of atrial fibrillation

2.2 Atrial fibrillation in a district general hospital

CHAPTER 3 THROMBOGENESIS IN CHRONIC ATRIAL

FIBRILLATION

3.1 Studies of plasma fibrinogen, von Willebrand factor and fibrin D-dimer in chronic atrial fibrillation

CHAPTER 4 THROMBOGENESIS AND CARDIOVERSION OF ATRIAL FIBRILLATION

4.1 Anticoagulation and cardioversion of atrial fibrillation

4.2 Studies of plasma fibrinogen and D-dimer in patients with atrial fibrillation: The effects of cardioversion to sinus rhythm 
CHAPTER 5 THROMBOGENESIS IN PAROXYSMAL ATRIAL

FIBRILLATION

5.1 A review of the management of paroxysmal atrial fibrillation

5.2 Studies of plasma fibrinogen and D-dimer in paroxysmal atrial fibrillation

\section{CHAPTER 6 THROMBOGENESIS IN PAROXYSMAL SUPRA-}

VENTRICULAR TACHYCARDIA

6.1 Studies of plasma fibrinogen and D-dimer in paroxysmal supraventricular tachycardia

\section{CHAPTER 7 THROMBOGENESIS IN VALVE DISEASE}

7.1 Influence of heart valve disease on thromboembolic risk

7.2 Studies of plasma fibrinogen and D-dimer in mitral regurgitation and aortic stenosis

CHAPTER 8 THROMBOGENESIS IN LEFT VENTRICULAR SYSTOLIC DYSFUNCTION

8.1 Is systolic dysfunction important in thrombogenesis?

8.2 Studies of fibrinogen, von Willebrand factor and D-dimer in left ventricular systolic dysfunction

\section{CHAPTER 9 THROMBOGENESIS IN LEFT VENTRICULAR}

DIASTOLIC DYSFUNCTION

9.1 Is diastolic dysfunction important?

9.2 Studies of fibrinogen, von Willebrand factor and D-dimer in left ventricular diastolic dysfunction

\section{CHAPTER 10 ANTICOAGULATION FOR ATRIAL FIBRILLATION}

\section{AND LEFT VENTRICULAR DYSFUNCTION}

10.1 Anticoagulation for chronic atrial fibrillation, left ventricular dysfunction and left ventricular aneurysms

10.2 Studies of plasma fibrinogen and D-dimer in patients with chronic atrial fibrillation, left ventricular dysfunction and left 
ventricular aneurysms: The effects of introducing

anticoagulant therapy

CHAPTER 11 LIPOPROTEIN (a) AND THROMBOGENESIS IN

ATRIAL FIBRILLATION AND LEFT VENTRICULAR

DYSFUNCTION

11.1 Lipoprotein (a) in chronic atrial fibrillation and left

ventricular dysfunction

CHAPTER 12 CONCLUDING REMARKS AND FUTURE

DIRECTIONS

APPENDIX 1 Publications and presentations arising from

work of this thesis

296

APPENDIX 2 References 


\section{LIST OF TABLES}

PAGE NO

CHAPTER 1

1.1 Plasma fibrinogen levels $(\mathrm{g} / \mathrm{l})$ in patients with coronary artery disease

30

CHAPTER 3

$\begin{array}{ll}\text { 3.1 Chronic atrial fibrillation - Patient characteristics } & 89\end{array}$

3.2 Plasma fibrinogen, von Willebrand factor and D-dimer in chronic atrial fibrillation $\quad 90$

$\begin{array}{ll}\text { 3.3 Echocardiography in chronic atrial fibrillation } & 91\end{array}$

3.4 Spearman correlations with age, cardiac dimensions and left ventricular function $\quad 92$

3.5 Intra- and between-observer repeatability for 2-dimensional and M-mode

$\begin{array}{ll}\text { echocardiography } & 93\end{array}$

\section{CHAPTER 4}

4.1 Factors affecting the ease of cardioversion of atrial fibrillation and maintenance of sinus rhythm

4.2 Cardioversion of atrial fibrillation - Patient characteristics

4.2 Plasma fibrinogen levels and cardioversion of atrial fibrillation

4.3 Plasma fibrin D-dimer levels and cardioversion of atrial fibrillation

CHAPTER 5

5.1 Paroxysmal atrial fibrillation and plasma fibrinogen levels (whole study group)

5.2 Plasma fibrinogen and D-dimer in paroxysmal atrial fibrillation (no warfarin)

5.3 Echocardiography and paroxysmal atrial fibrillation (no warfarin group)

5.4 Paroxysmal atrial fibrillation - Spearman Correlations

\section{CHAPTER 6}

6.1 Plasma fibrinogen and fibrin D-dimer in paroxysmal supraventricular tachycardia 


\section{CHAPTER 7}

7.1 Plasma fibrinogen and D-dimer in mitral regurgitation and aortic stenosis

\section{CHAPTER 8}

8.1 Echocardiographic indications of left ventricular thrombi 188

8.2 Indications for anticoagulant therapy for possible left ventricular thrombi $\quad 188$

8.3 Left ventricular systolic dysfunction - Patient characteristics 200

$\begin{array}{ll}\text { 8.4 Left ventricular function and prothrombotic factors } & 201\end{array}$

8.5 Aspirin and plasma fibrinogen \& D-dimer levels 202

8.6 Left ventricular systolic dysfunction - Echocardiography data 203

8.7 Left ventricular systolic dysfunction - Spearman correlations 205

\section{CHAPTER 9}

9.1 Left ventricular diastolic dysfunction-Patient characteristics and Doppler parameters 231

9.2 Diastolic dysfunction and thrombogenic factors 233

9.3 Spearman correlations with Doppler characteristics of diastolic dysfunction 235

9.4 Effects of aneurysm, left ventricular systolic dysfunction and diastolic dysfunction (and their interaction) on prothrombotic factors 241

9.5 Intra- and between-observer repeatability for Doppler echocardiography 242

CHAPTER 10

$\begin{array}{ll}10.1 \text { Trials of anticoagulation in atrial fibrillation } & 265\end{array}$

10.2 Anticoagulant therapy - Patient characteristics 266

$\begin{array}{ll}\text { 10.3 Plasma fibrin D-dimer and anticoagulation } & 267\end{array}$

$\begin{array}{ll}\text { 10.4 Plasma fibrinogen levels and anticoagulation } & 268\end{array}$

10.5 Spearman correlations between plasma D-dimer levels and anticoagulation $\quad 269$

CHAPTER 11

11.1 Chronic atrial fibrillation and lipoprotein (a) 282

11.2 Left ventricular dysfunction and lipoprotein (a) 283

11.3 Left ventricular diastolic dysfunction and lipoprotein (a) 284 


\section{LIST OF FIGURES}

PAGE NO

\section{CHAPTER 1}

1.1 Plasma fibrinogen and

(a) the coagulation and fibrinolytic systems; (b) atherogenesis

\section{CHAPTER 2}

2.1 Emergency admissions with atrial fibrillation to a district general hospital (6 months) 72

2.2 Underlying causes of atrial fibrillation 73

2.3 Presenting clinical features on admission 73

\section{CHAPTER 3}

3.1 2-dimensional Echocardiography: Parasternal long axis view, with anteroposterior dimension (D1) for left atrium (LA)

3.2 2-dimensional Echocardiography: Apical 4-chamber view; mediolateral (D2) and superoinferior (D3) dimensions for :

(a) left atrium (LA) and (b) right atrium (RA)

3.3 Ellipsoid formula used for calculation of atrial volumes

3.4 M-Mode Echocardiography of left ventricle (LV) with septum (A), LV end-diastolic dimension (B),LV posterior wall (C) and LV end-systolic dimension (D) respectively 99

3.5 Plasma fibrinogen and chronic atrial fibrillation

3.6 Plasma D-dimer and chronic atrial fibrillation

3.7 Plasma von Willebrand factor and chronic atrial fibrillation

\section{CHAPTER 4}

4.1 Cardioversion in atrial fibrillation (no warfarin group) and

(a) plasma fibrinogen; (b) D-dimer

4.2 Cardioversion in atrial fibrillation (warfarin group) and

(a) plasma fibrinogen; (b) D-dimer

\section{CHAPTER 5}

5.1 Plasma fibrinogen and paroxysmal atrial fibrillation

5.2 Plasma D-dimer and paroxysmal atrial fibrillation 


\section{CHAPTER 6}

6.1 Plasma fibrinogen and paroxysmal supraventricular tachycardia

6.2 Plasma D-dimer and paroxysmal supraventricular tachycardia

\section{CHAPTER 7}

7.1 An example of Colour flow Doppler echocardiography demonstrating mitral regurgitation

7.2 An example of Continuous wave Doppler echocardiography demonstrating mixed aortic valve disease

7.3 Plasma fibrinogen and valve disease

7.4 Plasma D-dimer and valve disease

\section{CHAPTER 8}

8.1 Plasma fibrinogen and left ventricular function

8.2 Plasma D-dimer and left ventricular function

8.3 Plasma von Willebrand factor and left ventricular function

\section{CHAPTER 9}

9.1 Determinants of left ventricular diastolic function

9.2 Relationship between systolic and diastolic dysfunction in ischaemic heart disease

9.3 An example of Pulsed wave Doppler echocardiography demonstrating mitral inflow velocity pattern

9.4 Echocardiographic patterns of diastolic dysfunction

CHAPTER 10

10.1 Anticoagulation in atrial fibrillation and

(a) plasma fibrinogen; (b) D-dimer

10.2 Anticoagulation in left ventricular aneurysms and

(a) plasma fibrinogen; (b) D-dimer

CHAPTER 11

11.1 Serum lipoprotein (a) and atrial fibrillation

11.2 Plasma lipoprotein (a) and left ventricular function 


\section{DECLARATION}

This thesis is a record of work conducted by the author at the Department of Cardiology, Stobhill General Hospital. This work has not previously been accepted for a higher degree. A list of publications and papers presented to learned societies arising from work contained in this thesis is given in Appendix 1.

I have been fortunate in having the co-operation of a number of colleagues who are formally acknowledged. The work of the thesis has been carried out by me, and the writing of it is entirely my own work.

Gregory - Yoke Hong LIP 


\section{ACKNOWLEDGEMENTS}

I am extremely grateful to the following people without whose unstinting help/patience this thesis would not have been possible:

Dr Francis G Dunn for his support and advice in conducting this research and for allowing me access to the facilities of at the Department of Cardiology, Stobhill General Hospital.

Professor Gordon D O Lowe for his support and advice in conducting this project and for allowing me access to the facilities at the Haemostasis and Thrombosis Unit, University Department of Medicine, Royal Infirmary, Glasgow. In addition, I wish to thank him for his continued help and encouragement, and for stimulating my interest in thrombogenesis.

Dr Malcolm J Metcalfe for his continued encouragement during my work.

I would also like to thank Dr Alan P Rae and Dr Calvin Tean, for their helpful comments.

Finally, I would also like to acknowledge the help of the following people: Ann Rumley, Dr Hiliary Rolton, Dr Elizabeth Farish and Duncan Smith (technical assistance); Dr M Malekianpour (echocardiography inter-observer variation); Dr James Curral (statistical advice).

This thesis is dedicated to Peck Lin whose love, patience and support have sustained me during its preparation. 


\section{SUMMARY}

Identification of patients at risk of thromboembolism poses a daunting

problem. Nevertheless the availability of measurable plasma or serum factors may help to identify those patients at particular risk of thrombogenesis and whom may benefit from prophylactic anticoagulant therapy. My thesis investigates the roles of fibrinogen, fibrin turnover, endothelial dysfunction and lipoprotein(a) in thrombogenesis associated with two cardiovascular conditions at high thromboembolic risk, that is, atrial fibrillation and left ventricular dysfunction.

Atrial fibrillation was found to be a common cardiac arrhythmia in a survey of emergency admissions over a six month period to my hospital. Heart failure and cerebrovascular events were the commonest presenting features, accounting for about half of all emergency admissions who were found to be in atrial fibrillation. This survey gave a comprehensive profile of how patients with atrial fibrillation were investigated and managed in a district general hospital. For example, a suboptimal application of standard investigations in patients with atrial fibrillation was identified together with a reluctance to perform cardioversion or to commence anticoagulant therapy. This being despite the increasingly important roles for these two therapeutic manoeurres in the current management of atrial fibrillation. One possible reason for the low rate of introducing anticoagulation may be the uncertainty of many physicians in identifying patients at high thromboembolic risk. The availability of a suitable plasma marker of thrombogenesis may assist decision-making in these patients.

Factors such as fibrinogen, von Willebrand factor and lipoprotein (a) have been associated with thrombosis, embolism and stroke. In addition, the fibrin D-dimer fragment is a marker of fibrin turnover and is indirectly a measure of intravascular thrombus formation. In my study, I have identified abnormalities of these plasma markers suggesting a prothrombotic state in patients with atrial fibrillation and left ventricular aneurysms.

Patients with chronic atrial fibrillation, for example, have significant elevations of plasma fibrinogen and von Willebrand factor when compared to normal population values; and this is irrespective of aspirin or warfarin therapy. Those who are on no antithrombotic 
therapy have the highest levels of plasma fibrin D-dimer, suggesting the highest levels of intravascular fibrin turnover in these patients. In contrast, patients whom are established on warfarin have the lowest fibrin D-dimer levels, consistent with the beneficial effect of warfarin in reducing thrombogenesis. No significant effect on these factors with respect to atrial size, ventricular function (as measured by echocardiography), or underlying valve or ischaemic heart disease was noted.

Cardioversion of atrial fibrillation to sinus rhythm replaces the irregular atrial activity of atrial fibrillation with the regular atrial systole of sinus rhythm. If thrombogenesis has a simple mechanical basis, cardioversion of atrial fibrillation should be expected to immediately normalise plasma fibrinogen and fibrin D-dimer levels. This does not appear to be the case, as in patients without warfarin therapy, plasma fibrin D-dimer falls sequentially over two weeks following cardioversion. This is partly due to the clearance of D-dimer from the circulation and that atrial systole may not resume immediately. In addition, there is little change in plasma fibrinogen levels, which may inpart be due to the individual variation in the return of atrial systole. Although clinical studies suggest a 'high risk' period for thromboembolism following cardioversion, no significant peak in plasma fibrinogen or fibrin D-dimer has been noted. This is in keeping with the hypothesis that any emboli are due to pre-formed thrombus rather than formation of new thrombus following cardioversion. Patients who are anticoagulated pre- and post-cardioversion have low plasma D-dimer levels, which do not alter over the two weeks' follow-up, consistent with the prophylactic effects of warfarin in reducing thromboembolic risks during cardioversion.

If fibrin D-dimer is a suitable indicator of intravascular thrombogenesis, further evidence may be obtained by a study of patients with paroxysmal atrial fibrillation. These patients are regarded as at intermediate thromboembolic risk when compared to chronic atrial fibrillation and those in sinus rhythm. Patients with paroxysmal atrial fibrillation (on no warfarin therapy) indeed do have intermediate levels of plasma fibrinogen and fibrin D-dimer, in keeping with clinical observations. This suggests that flow abnormalities may be important in thrombogenesis, as sustained irregular atrial activity (in chronic atrial fibrillation) causes greater abnormalities in markers of thrombogenesis when compared to patients with intermittent 
irregular atrial contractions (for example, paroxysmal atrial fibrillation) and those with normal, regular atrial systolic function (in sinus rhythm).

Patients with paroxysmal supraventricular tachycardia (whom have a paroxysmal tachycardia with normal regular atrial contractions) were also compared with matched patients manifesting paroxysmal atrial fibrillation. In this small study, patients with paroxysmal supraventricular tachycardia had lower levels (similar to population values) of plasma fibrinogen and fibrin D-dimer when compared with matched patients having paroxysmal atrial fibrillation. This again suggests a role for flow abnormalities associated with atrial fibrillation in influencing plasma fibrinogen and fibrin D-dimer levels.

Epidemiological evidence suggests that valvular heart abnormalities significantly add to the thromboembolic risk of patients with atrial fibrillation. All patients with valvular disease may not have similar risks of thromboembolism and this may in part be related to their inherent flow characteristics. I therefore studied a small series of patients with aortic stenosis and mitral regurgitation. Both groups had higher plasma fibrinogen levels when compared to population levels, in keeping with flow abnormalities in these patients. However, patients with mitral regurgitation have lower plasma D-dimer levels, indicating lower intravascular fibrin turnover. This finding is consistent with observations that the presence of mitral regurgitation reduces spontaneous echo contrast (associated with thrombus and embolism) and intraventricular thrombus formation (for example, in patients with dilated cardiomyopathy). This suggests that plasma D-dimer may be associated with stasis (allowing thrombus formation) within the atrial cavity which is reduced by the presence of mitral regurgitation. Patients with aortic stenosis, however, have similar plasma D-dimer levels to population values, in keeping with the different pathophysiology (calcific emboli and platelets) of emboli associated with aortic stenosis.

Epidemiological evidence also suggests that a further factor contributing to the risk of stroke in atrial fibrillation is the presence of heart failure. Patients with ischaemic heart disease have higher plasma fibrinogen and von Willebrand factor levels when compared with population controls, but those with left ventricular aneurysms (as defined by radionuclide ventriculography), however, have the highest levels of plasma fibrinogen, fibrin D-dimer and 
von Willebrand factor. This indicates the highest levels of intravascular thrombogenesis in patients with left ventricular aneurysms and may explain the association of heart failure (associated with left ventricular impairment) and thromboembolic events. As in patients with chronic atrial fibrillation, patients with left ventricular aneurysms who were established on warfarin therapy have low fibrin D-dimer levels, consistent with a reduction in thrombogenesis.

Clinical or epidemiological studies investigating the contribution of heart failure to thromboembolic risk do not, however, make a distinction between systolic and diastolic dysfunction. This is pertinent as $30 \%$ or more of admissions with clinical heart failure have normal systolic function. There were, however, no differences in plasma fibrinogen, fibrin D-dimer and von Willebrand factor between patients with and without diastolic dysfunction. This suggests that the flow abnormalities associated with systolic (rather than diastolic) impairment have the greatest influence upon fibrinogen and fibrin turnover (and thrombogenesis).

Recent prospective studies of patients with nonvalvular atrial fibrillation have shown that prophylactic anticoagulant therapy is beneficial in reducing thromboembolic risk. In patients with chronic atrial fibrillation and left ventricular aneurysms in whom anticoagulant therapy is introduced, there is a sequential reduction in plasma fibrin D-dimer (but not fibrinogen) levels with the introduction of warfarin therapy. In addition, in those with the highest initial D-dimer levels $(>100 \mathrm{ng} / \mathrm{ml})$ this reduction is highly significant. This is again consistent with the beneficial effect of warfarin in reducing stroke and thromboembolism, and patients at highest risk (e.g. D-dimer $>100 \mathrm{ng} / \mathrm{ml}$ ) may be selected by measurement of plasma fibrin D-dimer levels.

Lipoprotein (a) is another factor associated with thrombogenesis, especially in ischaemic heart disease. I therefore measured lipoprotein (a) levels in the two conditions in which the highest intravascular fibrin turnover was demonstrated, those being chronic atrial fibrillation and left ventricular aneurysms. There were, however, no significant differences between patients and healthy controls and no alteration even with warfarin therapy. This is consistent with observations that lipoprotein (a) is predominantly genetically determined, and varies little with environmental influences. 
In summary, plasma levels of fibrinogen, D-dimer and von Willebrand factor are elevated in patients with chronic atrial fibrillation (without antithrombotic therapy) and patients with left ventricular aneurysms. Intermediate levels are seen in patients with paroxysmal atrial fibrillation. My thesis therefore suggests possible roles for fibrinogen, increased fibrin turnover (as measured by plasma D-dimer), endothelial dysfunction (as reflected by von Willebrand factor) and rheological abnormalities in thrombogenesis in these patients. Lipoprotein (a) appears to be little changed in these patients, with no relationship to other plasma factors, in keeping with the strong genetic influence of this prothrombotic factor. in addition, levels of these markers in patients with atrial fibrillation were not significantly influenced by cardiac chamber size and structural abnormalities; whilst in patients with left ventricular dysfunction, the presence of left ventricular aneurysms and systolic (rather than diastolic) dysfunction was found to be important.

Therapeutic interventions may also alter thrombogenesis. For example, cardioversion and anticoagulation both reduce plasma D-dimer levels, suggestive of the beneficial effects of these interventions. As my survey of admissions with atrial fibrillation demonstrated however that both of these procedures are infrequently considered, the availability of a suitable plasma marker to select patients at high risk (who would benefit most from intervention) may aid a physician in considering these interventions more frequently. My thesis suggests that measurement of plasma fibrin D-dimer may provide such a marker of thrombogenesis. Measurement of other factors such as fibrinogen and von Willebrand factor will also contribute to the overall risk profile, allowing the risk of thrombogenesis in such patients to be predicted. 


\section{LAYOUT OF THESIS}

The aim of this study was to investigate the haemo-rheological mechanisms of thrombogenesis in two cardiac conditions at high risk of thromboembolism: atrial fibrillation and left ventricular dysfunction.

In the introduction in CHAPTER 1,1 review the current literature on the role of haemorheological factors and lipoprotein (a) in thrombogenesis. I also summarise the background and aims of my thesis. In CHAPTER 2, I undertake a survey of emergency admissions with atrial fibrillation to a district general hospital. This survey gave a comprehensive profile of admissions with atrial fibrillation and how they are investigated or managed, allowing the identification of suitable patients for further study. In CHAPTER 3 patients with chronic atrial fibrillation are studied. This is a group of 85 patients with chronic atrial fibrillation (divided into those on aspirin, warfarin and no antithrombotic therapy), whom are compared to population controls. Flow abnormalities in atrial fibrillation may contribute to thrombogenesis in these patients and conversion to sinus rhythm may alter this process. Therefore, patients whom were cardioverted from atrial fibrillation into sinus rhythm are studied in CHAPTER 4. The results of these studies stimulated an investigation into a group of patients with paroxysmal atrial fibrillation whom are at intermediate thromboembolic risk (CHAPTER 5). In CHAPTER 6 patients with paroxysmal supraventricular tachycardia are studied allowing comparison with patients with paroxysmal atrial fibrillation.

The presence of valve disease and left ventricular dysfunction are risk factors for thromboembolism and are additive to the risks of thrombogenesis in atrial fibrillation. In CHAPTER 7, the influence of heart valve disease is studied, whilst patients with left ventricular dysfunction are studied in CHAPTER 8. Current epidemiological studies do not differentiate between the effects of systolic and diastolic dysfunction in assessing thromboembolic risk. These patients with diastolic dysfunction are therefore studied in CHAPTER 9. The effects of introducing anticoagulation in patients with chronic atrial fibrillation and left ventricular aneurysm are studied in CHAPTER 10. CHAPTER 11 addresses the contribution of lipoprotein (a) to the mechanisms of thrombogenesis. 
CHAPTER 12 comprises a discussion of the overall results, an examination of the interrelationship of factors studied in this thesis and concludes with a summary of the main findings of my thesis and suggestions for further study.

In summary, my thesis will commence with a review of the literature on plasma markers of thrombogenesis. This will follow with clinical studies set out as individual chapters. Each of these will include methodology used, results obtained and a critical discussion including relevant literature review. 
CHAPTER 1

\section{INTRODUCTION}




\subsection{THROMBOGENESIS AND PROTHROMBOTIC FACTORS}

\section{Introduction}

Many cardiovascular conditions are associated with an increased thromboembolic risk: two notable ones being atrial fibrillation and poor left ventricular function. The mechanisms of thromboembolism in these two common conditions have not been fully elucidated. These mechanisms are likely to be complex, multifactorial and inter-related. For example, the presence of a dilated left atrium and poor left ventricular function are considered to be contributory risk factors to the thromboembolic risk in atrial fibrillation. In patients in sinus rhythm, however, the presence of poor left ventricular function (particularly in the presence of an associated left ventricular aneurysm) is also associated with an increased thromboembolic event rate. A possible mechanism common to both conditions is the presence of slow, sluggish or recirculating blood flow within a dilated left atrial cavity or a poorly contractile left ventricle (or aneurysm), which may result in an increased propensity toward clot or thrombus formation (thrombogenesis).

How does altered flow and rheology relate to this thromboembolic risk, and how can we quantify this risk?

The role of blood flow properties (rheology) in thrombogenesis has been long recognised. More than a century ago, Virchow (1856) postulated that a triad of abnormalities were key determinants of the process of thrombus formation: (i) slowing of blood flow; (ii) changes in the composition of the blood itself (that is, blood cells and clotting factors); and (iii) changes in (and blood interactions with) the blood vessel wall. A modern modification of this hypothesis suggests that turbulent blood flow, chronic damage to the endothelium (by injury from cigarette smoking and hypertension) and abnormalities in blood lipids result in functional abnormalities of the endothelium, leading to adhesion of monocytes and platelets, and the further accumulation of lipids. Continuation of this process leads to atheroma formation (atherogenesis). The process of arterial thrombogenesis is therefore very closely related to atherogenesis: it follows that specific thrombogenic factors may play key 
roles in the process of atherosclerotic lesion formation. Further evidence for this is the presence of platelets, fibrinogen, fibrin and fibrin degradation products in atheromatous plaques (especially at areas of thrombus formation) and the promotion of smooth muscle proliferation and lipid deposition by accumulated fibrinogen and fibrin (Hunt 1990).

The precise contributory factors to thrombogenesis and atherogenesis have, however, not been fully defined. Based on current clinical and epidemiological evidence, plasma factors, such as fibrinogen, fibrin D-dimer, von Willebrand factor and lipoprotein (a) may be suitable markers of thrombogenesis and atherogenesis; thus suggesting possible roles for increased fibrin formation, endothelial dysfunction and rheological disturbance in the mechanisms of promoting thrombus formation (thus contributing towards a prothrombotic or hypercoagulable state).

What then is the relative importance of these plasma prothrombotic factors in thrombogenesis and atherogenesis? Most evidence to date is from epidemiological studies. Increased plasma fibrinogen levels have been associated with an increase in risk of cardiovascular disease, including ischaemic heart disease, stroke and other thromboembolic events (Ernst et al 1992, Ernst and Resch 1993). Plasma fibrinogen is, however, also involved in blood coagulation and is an important determinant of blood viscosity and hence of blood flow (Lowe 1986). In the process of thrombus formation, fibrinogen is converted to fibrin by the action of thrombin, and the resulting fibrin monomers polymerise to form a soluble gel of non-crosslinked fibrin. The latter is then crosslinked by thrombin-activated Factor XIII to form an insoluble fibrin clot. Subsequent activation of the fibrinolytic system results in formation of the enzyme plasmin, which cleaves both fibrinogen and fibrin to yield fibrin degradation products (FDPs). Of these, only the degradation products from crosslinked fibrin (XL- FDP) contain the D-dimer fragment. The process of fibrin turnover can therefore be assessed by the measurement of plasma fibrin D-dimer levels, which are therefore an indirect marker of intravascular thrombus formation (thrombogenesis).

The blood vessel wall also contributes to thrombogenesis (Virchow's triad) and any endothelial disturbance can be assessed by measurement of plasma levels of von Willebrand factor (VWF), which is an increasingly recognised marker of endothelial dysfunction 
(Blann et al 1993, Jansson et al 1991). Another serum factor, lipoprotein (a) [Lp(a)], has been recognised for nearly 30 years as a partially genetically-determined lipoprotein which is associated with an increase in atherogenesis and thrombogenesis. Elevated $\mathrm{Lp}$ (a) levels have been associated with an increased risk for coronary artery disease, stroke and restenosis after coronary artery bypass surgery (Rees 1991, Scott 1991). The actual function of Lp(a), however, remains largely unknown. In vitro studies have shown that $L p(a)$ competes in equimolar concentrations with plasminogen for binding to plasminogen receptors on vascular endothelial cells, resulting in an inhibitory effect on endogenous fibrinolysis. $L p(a)$ may also deliver cholesterol to proliferating cells by binding fibrin at sites of vascular injury, therefore contributing to thrombus and atheroma formation (Lawn 1992).

The potential interactions between the plasma markers of thrombogenesis and atherogenesis are, therefore, complex. Their relative importance as useful markers remains to be demonstrated in prospective studies. Improved biochemical techniques have, however, allowed the easy measurement of these key factors in thrombogenesis. Study of the possible mechanisms of thrombogenesis in cardiac conditions with particular risk of thromboembolism is therefore feasible. Measuring and evaluating such markers of an apparent intravascular thrombogenic state may also be of value in clinical practice. For example, measurement of these plasma factors may allow screening of patients in order to identify those most at risk of thromboembolism and hence those whom might benefit most from antithrombotic therapy. Additionally, in the acute stage of a thrombotic occlusion (coronary arteries in the case of myocardial infarction, or in cerebrovascular events) these plasma factors may provide prognostic information and allow therapeutic monitoring. Such measurements also open the possibility of developing new strategies in antithrombotic therapy. 


\subsection{FIBRINOGEN, FIBRIN D-DIMER FRAGMENT, VON WILLEBRAND} FACTOR AND LIPOPROTEIN (a) IN CARDIOVASCULAR DISEASE:

\section{A REVIEW OF THE LITERATURE}

Introduction

The process of thrombus formation involves endothelial injury, platelet adherence, aggregation and release, thrombin generation and fibrin formation. In addition, thrombogenesis is closely associated with atheroma formation and both processes contribute to atherosclerotic cardiovascular disease. Interestingly, the hypothesis that thrombosis may contribute significantly to spontaneous atherogenesis was originally proposed by Rokitansky (1852), who postulated that atheroma formation results from intimal deposition of blood components, including thrombi and fibrin. There is now increasing pathological evidence that the progression of atherosclerosis is, probably to a significant extent, closely related to ongoing mural thrombus formation ('thrombogenesis') and organisation (Duguid 1946, Woolf and Davies 1992).

Plasma factors involved in the process of thrombogenesis which have been studied include plasma fibrinogen, factor VII, fibrin D-dimer fragments and plasma von Willebrand factor. In addition, plasma lipoprotein (a) has been shown to related to the intrinsic fibrinolytic tendency of the body, although the precise role of this lipoprotein and its relationship to other coagulation factors and thrombogenesis remains unclear (Figure 1.1).

\section{PLASMA FIBRINOGEN.}

Biochemistry and pathophysiology

Human fibrinogen is a large glycoprotein (340000 Daltons) composed of 3 pairs of nonidentical polypeptide chains (A alpha, B beta and gamma) which are joined together by disulphide bonds at their amino-acids. It is well recognised that fibrinogen is an important determinant of the rheological characteristics of blood flow, as well as platelet aggregability (Lowe 1986). Fibrinogen is also an essential component of the blood coagulation system, being the precursor of fibrin. However, at the 'usual' plasma levels of 1.5 
to $4.5 \mathrm{~g} / \mathrm{l}$ its concentration far exceeds the minimum concentration of $0.5-1 \mathrm{~g} / \mathrm{l}$ necessary for haemostasis.

It is postulated therefore that elevated plasma fibrinogen levels may reflect a prothrombotic or hypercoagulable state as it is a major determinant of fibrin formation, blood viscosity and platelet aggregation. This possibly enhanced thrombogenic tendency of fibrinogen may contribute to the development of the atheromatous lesion itself and the tendency of ruptured atheromatous plaques to occlude, for example, in coronary artery disease. In patients with an accelerated risk of atherosclerosis due to hyperlipidaemia, hypertension or diabetes, this is particularly important and raised plasma fibrinogen levels may be an independent and major additive risk factor to such patients (Ernst et al 1992). It is also proposed that elevated plasma fibrinogen may promote atherosclerotic vascular damage as high concentrations of fibrinogen and fibrin have been found in developing atherosclerotic lesions (Bini and Kudryk 1992, Smith et al 1990). The pathogenesis of such lesions is not solely due to fibrinogen and fibrin but likely to involve increased fibrin formation and degradation (Bini and Kudryk 1992), platelet aggregation (which fibrinogen increases) and changes in blood viscosity (which fibrinogen promotes) (Lowe 1986) (Figure 1.1).

The importance of the intimate interaction between plasma fibrinogen and platelets in thrombogenesis is suggested by the process of platelet recruitment. This involves the expression of the glycoproteins $\mathrm{llb} / \mathrm{llla}$ receptor for fibrinogen and other cytoadhesive proteins that include von Willebrand factor (Badimon et al 1992, Harker 1987). In addition, patients with poor left ventricular function have greater platelet activation (as reflected by increased plasma levels of factors which reflect platelet activity, such as platelet factor 4 and $B$ thromboglobulin), and together with an interaction with plasma fibrinogen, thrombogenesis may therefore be promoted (Schmitz-Huebner et al 1988). Similarly, patients with previous myocardial infarction demonstrate significant elevations in platelet factors due to the reaction of platelets with previously infarcted myocardium (rather than arteriosclerotic coronary arteries) (Nichols et al 1982). This suggests that enhanced platelet activation may be associated with the presence of left ventricular wall motion abnormalities (Nichols et al 1982). 


\section{COAGULATION}

\section{EXTRINSIC}

Tissue Factor VII

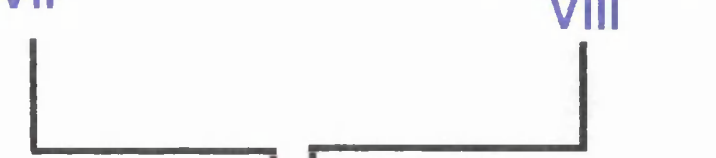

Protein C Protein S
FIBRINOLYSIS

EXTRINSIC INTRINSIC tPA

PAI

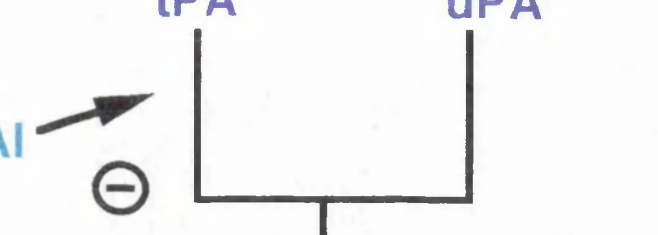

\section{Prothrombin}

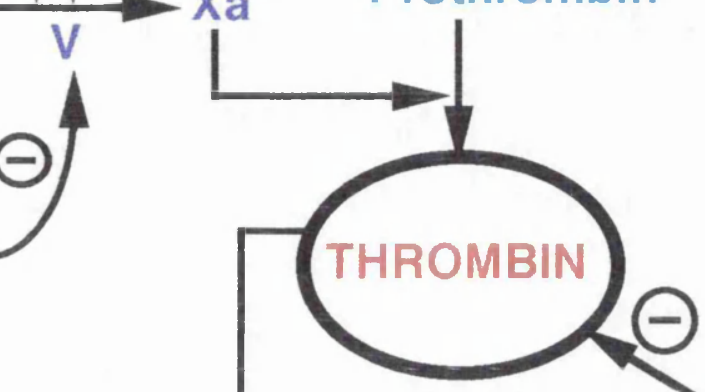

Anti-

PLASMINOGEN

thrombin

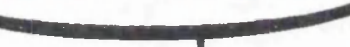

$\ominus$

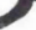

anti-

plasmin

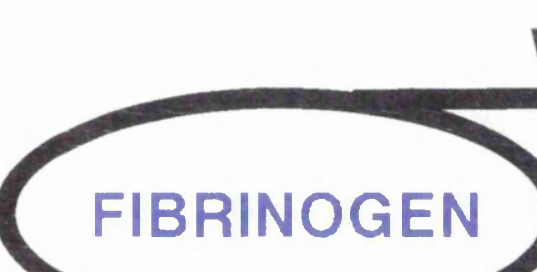

WW=von Willebrand factor;

Endothelial

disturbance

\section{FIBRINOGEN}

vWF

platelet

adhesion

and

aggregation

Blood

viscosity

\section{THROMBOSIS}

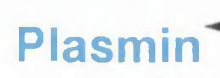

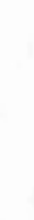




\section{FIBRINOGEN}

- Stimulation of migration of smooth muscle cells

- Accumulation of apo-B containing lipoproteins

( eg. lipoprotein (a))

- Proliferation of smooth muscle

- Binding to platelet receptors
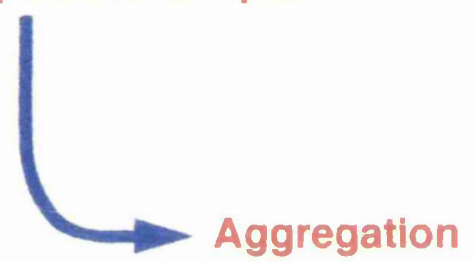

Release of

- ADP

- 5-HT

- PF4

- PDGF

Plasma coagulation

\section{Plasma}

Blood rheology

Erythrocytes

Leucocytes

FIGURE 1.1

FIBRINOGEN AND ATHEROGENESIS

$(\mathrm{ADP}=$ adenosine diphosphate; $5-\mathrm{HT}=5$-hydroxytryptamine; $\mathrm{PF} 4=$ platelet factor 4 ;

PDGF = platelet derived growth factor) 
Plasma fibrinogen is an important contributory factor to the flow properties of the blood and is a major determinant of plasma viscosity and red cell aggregation (and hence, whole blood viscosity). An elevation in whole blood viscosity may lead to sluggish blood flow, which is associated with thrombus formation. Not surprisingly, this rheological index is high in patients with coronary artery disease and patients at high risk of ischaemic heart disease (Mares et al 1991).

\section{Epidemiological studies}

Plasma fibrinogen has been well established as a cardiovascular risk factor. Studies from different countries over the last 10 years have demonstrated a significant association between plasma fibrinogen and cardiovascular mortality and morbidity, including ischaemic heart disease and stroke (Garcia-Bolao et al 1990, Green and Humphries 1989, Ernst 1991, Ernst and Resch 1993). In addition to elevated fibrinogen levels, plasma viscosity (to which plasma fibrinogen contributes) and leucocyte count are at least as predictive for cardiovascular events as 'traditional' cardiovascular risk factors such as cholesterol, diastolic blood pressure and body mass index (Yarnell et al 1991). However, plasma fibrinogen has other 'external' influencing factors, and it is recognised that concentrations change with age, post-menopausal state, obesity and smoking, and fall with alcohol consumption (as will be discussed below) (Ernst and Resch 1993, Green and Humphries 1989, Meade et al 1979).

In the Northwick Park Heart study of white men, plasma fibrinogen levels were higher in those who died of cardiovascular disease (Meade et al 1986). An important finding is that an elevation of one standard deviation in fibrinogen concentration (about $0.6 \mathrm{~g} / \mathrm{l}$ ) was associated with an $84 \%$ increase in the risk of ischaemic heart disease within the next 5 years (In contrast, the corresponding risk progression for cholesterol was only $43 \%$ for all age groups) (Meade et al 1986).

In the Framingham study, the risk for coronary heart disease was also significantly related to the plasma fibrinogen level (Kannel et al 1987). This risk however diminished with age in women but not in men. The converse was demonstrated for the risk of stroke, which was related to plasma fibrinogen levels in men but not in women. Similar results 
are demonstrated in the PROCAM study of 2187 patients whom were prospectively followedup for 48 months, where those with cardiac events had significantly elevated plasma fibrinogen levels (Heinrich et al 1991b). In the Gothenburg Study, there was also a clear relationship between plasma fibrinogen and the development of stroke and myocardial infarction, even after adjusting for blood pressure, serum cholesterol and smoking (Wilhelmsen et al 1984).

In the Caerphilly and Speedwell Collaborative Heart Study, a combined cohort of $\mathbf{4 8 6 0}$ middle-aged men was studied and yet again, plasma fibrinogen and viscosity, and white blood cell count were important risk factors for ischaemic heart disease (Yarnell et al 1991). These associations with ischaemic heart disease were independent of smoking status, although part of the adverse effects of smoking may be mediated through plasma fibrinogen, viscosity and white cell count (Yarnell et al 1991). Another British study, the Leigh general practice study of $\mathbf{2 9 7}$ males demonstrated that plasma fibrinogen was also a major independent coronary risk factor (Stone and Thorp 1985).

In the Scottish Heart Health Study, plasma fibrinogen was measured in 8824 patients aged 40-59 years and related to cardiovascular risk factors. This large study demonstrated that women had higher plasma fibrinogen levels than men, and that there was a positive association with age, smoking, total cholesterol and body mass index and a negative association with alcohol consumption (Lee et al 1990, Lee et al 1993). As in the Caerphilly and Speedwell Collaborative Heart Study, the findings of the Scottish Heart Health Study do suggest that a raised plasma fibrinogen level was one mechanism by which several risk factors (including smoking) promoted coronary heart disease (Lee et al 1990, Yarnell et al 1991). The Scottish Heart Health Study, in addition, was the first large random population study to demonstrate that plasma fibrinogen was raised in subjects with a family history of premature heart disease and with a personal history of hypertension, diabetes, angina, myocardial infarction or intermittent claudication (Lee et al 1993). The latter finding was confirmed in the Edinburgh Artery Study (Lowe et al 1993). 
Ischaemic heart disease

An important relationship clearly demonstrated in the epidemiological studies discussed above is the association between risk factors for ischaemic heart disease and plasma fibrinogen levels. However, do the changes in plasma fibrinogen and other plasma factors extend to all categories of patients with ischaemic heart disease? Plasma fibrinogen and fibrin degradation products are generally elevated in coronary artery disease, but these concentrations appear to depend on the severity and time since onset of disease (Malaia et al 1990). Plasma viscosity and fibrinogen levels are also higher in patients with unstable angina than in patients with stable angina (Leschke et al 1988), and in patients with more severe coronary artery disease (Lowe et al 1980). Importantly, the latter elevation was comparable to the increased values found in patients following acute myocardial infarction (Leschke et al 1988). The abnormal blood viscosity in unstable angina (related in part to abnormal plasma fibrinogen levels) may contribute to increasing myocardial ischaemia and the progression of angina by slowing capillary blood flow and diminishing oxygen delivery. These results also suggest that the increased plasma fibrinogen levels during acute myocardial infarction are not solely a reactive (or infarction-related) mechanism, but are part of the pathogenesis of unstable angina by a rheological reduction in coronary perfusion and the promotion of thrombus formation.

The relationship to the extent or severity of coronary artery disease is consistent. In 9 out of 10 studies, plasma fibrinogen levels correlated significantly with the degree of coronary artery disease (Table 1.1). In a Glasgow study, for example, there was a modest association between the extent of coronary atheroma and plasma fibrinogen levels (but no relationship between the severity of coronary artery disease and components of the fibrinolytic enzyme system) (Small et al 1987). Recent large studies have also confirmed the association between plasma fibrinogen and extent of coronary atherosclerosis (ECAT Angina Pectoris Study Group 1993). The converse was demonstrated in one larger study of 225 patients, where plasma fibrinogen, von Willebrand factor and other clotting factors did not contribute independently to the prediction of an angiographic score that indicated the extent of coronary artery disease (Schmitz-Huebner et al 1988). While the trend is clear, care has to 
be taken in the interpretation of the absolute values of plasma fibrinogen summarised in Table 1.1 due to regional variations in plasma fibrinogen levels (Ernst 1991, Machin and Mackie 1993).

Despite the variation in time course and severity of ischaemic heart disease discussed above, an elevated plasma fibrinogen is nevertheless predictive of future cardiovascular events in patients with coronary heart disease (Dormandy et al 1982, Martin et al 1991), peripheral vascular disease (Banerjee et al 1992, Dormandy et al 1973, Fowkes et al 1993) and survivors of a first stroke (Ernst et al 1991).

Atrial fibrillation

Atrial fibrillation is commonly associated with stroke and thromboembolic events and associated rheological abnormalities may be a significant contributing factor. Indeed, plasma fibrinogen levels are elevated in patients with chronic atrial fibrillation (Kumagai et al 1990). The role of plasma fibrinogen in contributing to thrombogenesis in atrial fibrillation (and the effects of interventions such as anticoagulation and cardioversion to sinus rhythm) is further investigated by my thesis. In patients with chronic atrial fibrillation after threadmill exercise, plasma fibrinogen levels are increased in association with enhanced platelet activity but lower levels of antifibrinolytic activity (Furui et al 1987). This suggests therefore that haemorheological abnormalities in atrial fibrillation are 'real' and dynamic; and that the risk of thromboembolic complications may be enhanced by stress or physical exercise. 
TABLE 1.1

PLAsma fibrinogen leVels (g/l) IN PATIENTS WITH

\begin{tabular}{|c|c|c|c|}
\hline & $\begin{array}{r}\text { CORONARY } \\
\text { one-vessel disease }\end{array}$ & $\begin{array}{l}\text { TERY DISEASE } \\
\text { two-vessel disease }\end{array}$ & three-vessel disease \\
\hline Baller et al 1991 & 3.16 & 3.43 & 3.40 \\
\hline $\begin{array}{l}\text { Broadhurst et al } \\
1990\end{array}$ & 2.64 & 3.07 & 3.31 \\
\hline $\begin{array}{l}\text { ECAT Angina } \\
\text { Pectoris Study } \\
\text { Group } 1993\end{array}$ & 3.02 & 3.08 & $3.05-3.10$ \\
\hline Hamsten et al 1986 & 3.41 & 3.62 & 3.68 \\
\hline Handa et al 1989 & $\begin{array}{l}\text { M2.93 } \\
\text { F2.72 }\end{array}$ & $\begin{array}{l}\text { M3.13 } \\
\text { F3.05 }\end{array}$ & $\begin{array}{l}\text { M3.25 } \\
\text { F3.24 }\end{array}$ \\
\hline Leschke et al 1988 & 2.65 & 2.76 & 3.05 \\
\hline Lowe et al 1980 & 2.73 & 3.19 & 3.19 \\
\hline $\begin{array}{l}\text { Schmitz-Hubner et al } \\
1988\end{array}$ & 3.28 & 3.20 & 3.36 \\
\hline Small et al 1987 & 3.19 & 3.28 & 3.40 \\
\hline
\end{tabular}


Psychological and mental stress

The effect of psychological and mental stresses has been shown to have a significant effect on plasma coagulation and fibrinolysis, with demonstrable increased levels of plasma fibrinogen, von Willebrand factor and factor VIII coagulant activity (Jern et al 1989). These changes appear to be adrenergic in origin, being similar to responses observed during exercise and with an adrenaline infusion (Jern et al 1989). Mental stress has been regarded as an important risk factor for cardiovascular disease, and this study provides suggests one possible mechanism for this. Stress also induces an increase in platelet count and activity, and this (in combination with the increased levels of vWF, coagulation factors VII and VIII, and plasma fibrinogen discussed above) may have an additive effect in promoting occlusive thrombus formation. There is also experimental evidence that chronic mental stress promotes the development of atherosclerosis, which is a process requiring (as discussed above) fibrinogen, von Willebrand factor and platelets (Clarkson et al 1987).

\section{Cerebrovascular disease}

There is epidemiological evidence suggesting that plasma fibrinogen levels are significantly associated with cerebrovascular events. The Framingham and Gothenburg studies have both shown that plasma fibrinogen is an independent prognostic risk factor for stroke (Kannel et al 1987, Wilhelmsen et al 1984). Similarly, the Oxfordshire community stroke project demonstrated that an elevated plasma fibrinogen level (in addition to generalised hypercholesterolaemia and elevated low density lipoproteins) was a risk factor for prevalent cerebrovascular events (Qizilbash et al 1991). In addition, an analysis of risk factors for stroke in a cohort of 789 men born in 1913 demonstrated that increased plasma fibrinogen levels correlated significantly with the incidence of stroke (Welin et al 1987). Levels of plasma fibrinogen are also raised in patients with transient ischaemic attacks (Coull et al 1991, Qizilbash et al 1991).

There is also evidence of a link between these haemorheological parameters in cerebrovascular disease and histopathological evidence of atherosclerotic lesions associated with stroke. When patients with stroke were studied, an association between 
atherosclerotic lesions of the extracranial carotid artery and age, plasma fibrinogen and whole blood filterability was noted (Mercuri et al 1989). In addition, an elevated plasma fibrinogen predicted the progression of atherosclerotic carotid stenosis (Grotta et al 1989).

\section{Peripheral vascular disease}

Patients with peripheral vascular disease demonstrate abnormalities of several coagulation, fibrinolytic and rheological variables. For example, plasma fibrinogen concentration was an important independent predictor of coronary death in patients with intermittent claudication (Fowkes et al 1993). This finding is similar to that demonstrated in the Northwick Park prospective study of stable claudicants (Banerjee et al 1992). In the latter study, an increase in plasma fibrinogen of $1 \mathrm{~g} / \mathrm{l}$ was associated with a two-fold increase in the probability of death within the next six years (Banerjee et al 1992).

\section{Hypertension}

It is well recognised that hypertension adds to the risk factor profile for patients with coronary artery and cerebrovascular disease. In patients with hypertension, fibrinogen concentration and plasma viscosity are independent predictors of blood pressure. In a study of hypertensive diabetic Nigerians, for example, plasma fibrinogen was the strongest predictor of both systolic and diastolic blood pressure, followed by blood viscosity and body mass index (Mermeh 1990). In hypertensives with renoparenchymous and malignant forms of hypertension, marked rises of plasma fibrinogen are seen, with a linear correlation between the plasma-renin activity and the levels of systolic and diastolic blood pressure (Sonkodi et al 1979). This is pertinent as the haemorheological abnormalities in hypertension may have important implications for cardiovascular morbidity and mortality (Zannad and Stoltz 1992). An indication of these risks involved are demonstrated in the Leigh general practice study, where hypertensive subjects with plasma fibrinogen levels $>3.5$ g/l had a 12-fold higher cardiovascular risk than those with plasma fibrinogen levels $<2.9 \mathrm{~g} / \mathrm{l}$ (Stone and Thorp 1985). 
In addition, patients with hypertension and coronary artery disease are found to have higher levels of plasma fibrinogen and plasma viscosity than in patients with essential hypertension and normal coronary arteries (Leschke et al 1987, Leschke et al 1988, Leschke et al 1990). Importantly, however, hypertensive patients without coronary artery disease had significantly higher levels of plasma viscosity, red cell aggregation than did normotensive controls (Leschke et al 1987). These findings are of significance as the increased blood viscosity in hypertensive patients (both 'de novo' and secondary to increased plasma fibrinogen) may potentially contribute to angina pectoris and the reduction in coronary reserve observed in patients with hypertension and coronary artery disease (Leschke et al 1987. Leschke et al 1990).

\section{Diabetes}

Patients with diabetes mellitus are at increased risk of cardiovascular complications and interestingly, these patients also demonstrate abnormalities in plasma fibrinogen and other coagulation factors. In a study of 170 patients with Type II diabetes, plasma levels of fibrinogen were significantly higher than that of the normal population (Shihabi et al 1990). There was also a significant positive correlation between plasma fibrinogen and fasting glucose levels, serum cholesterol levels, glycosylated haemoglobin and urinary albumin excretion rates (Shihabi et al 1990). In the Framingham Study and Scottish Heart Health Study, diabetic subjects also had higher plasma fibrinogen concentrations than non-diabetic subjects (Kannel et al 1990, Lee et al 1993). These findings are important as elevated fibrinogen levels were a major determinant of cardiovascular complications in diabetics (Ganda and Arkin 1992, Kannel et al 1990).

One specific group of diabetics who are at particular cardiovascular risk are those who develop systemic complications such as renovascular disease and neuropathies. These patients also demonstrate significant plasma rheological and lipid abnormalities. In a case-control study of insulin dependant diabetics, patients with microalbuminuria (whom are those who tend to develop renal complications) had significant elevations of plasma fibrinogen, triglycerides, low density lipoprotein cholesterol, very low density lipoprotein 
cholesterol (but lower concentrations of high density lipoprotein cholesterol) when compared to controls (Jones et al 1989). Similarly when diabetic patients with neuropathies were studied, higher blood viscosity and elevations in coagulation factors including plasma fibrinogen and von Willebrand factor were found (Solerte et al 1988).

Plasma fibrinogen and lipid abnormalities therefore add significantly to the complex risk factor profile for cardiovascular disease in diabetic patients.

Body mass

In obese patients (Body Mass Index > 30) plasma viscosity and fibrinogen levels are significantly increased when compared to healthy subjects $(B M \mid<25)$ (Craverei et al 1987, Rosengren et al 1990). This increase is independent of the presence of atherosclerotic complications and other risk factors.

\section{Hormonal influence}

The incidence of myocardial infarction and cerebral thromboembolism is higher in women who use oral contraceptives and rheological abnormalities may partly explain this (Bogousslavsky and Regli 1987, Lidegaard 1993, RCGP Oral Contraception Study 1977, Shearman 1986). This patient group appears to have an increased thrombogenic tendency as measured by elevated platelet aggregation and plasma fibrinogen levels (Balleisen et al 1985, Garcia-Bolao et al 1990, Ernst et al 1989, Lowe et al 1980, Green and Humphries 1989). In the Scottish Health Heart Study, however, women who had previously been taking oral contraceptives had lower plasma fibrinogen levels when compared to those who had no such history, atthough multivariate analysis showed no independent association of history of oral contraceptive use and fibrinogen (Lee et al 1990).

Further evidence for a hormonal influence on plasma fibrinogen comes from the observation that levels are significantly higher in post menopausal women when compared to premenopausal women (Bonithon-Kopp et al 1990, Lee et al 1990, Meade et al 1983, Meade et al 1990). This increase in plasma fibrinogen (and Factor VIIc and cholesterol) seen in post-menopausal women has been calculated to increase the risk of fatal ischaemic 
heart disease by $40 \%$ (Meade et al 1983). The occurrence of the menopause was also accompanied with significant increases in plasma antithrombin III, factor VII coagulant activity, and factor VIIc, which were greatest in those experiencing a natural menopause when compared to those with an artificial menopause (Meade et al 1990). All these findings therefore may partly account for the increased cardiovascular complications seen in postmenopausal women.

\section{Smoking}

Smoking is a very important influencing factor for plasma fibrinogen levels. In fact, a dose-effect relationship exists between the number of cigarettes per day and plasma fibrinogen levels (Kannel et al 1987). All epidemiological studies to date have found the highest plasma fibrinogen levels amongst smokers.

For example, in the large (15000 men) WHO Co-operative trial of clofibrate, the highest plasma fibrinogen levels were seen in smokers (Green et al 1989). In an interesting cross-sectional population study from Copenhagen, a total of 439 men (all aged 51 years old) living in a certain area of Copenhagen were studied, and one of the strongest independent associations with plasma fibrinogen level was found for smoking (Møller and Kristensen 1991). A Swedish study of a cohort of 788 healthy men aged 54 years also showed that plasma fibrinogen positively correlated with cigarette smoking (Korsan-Bengtsen et al 1972). Similarly, in analysis of data from the Northwick Park Heart Study, there was a significant association between elevated plasma fibrinogen and ischaemic heart disease, and furthermore, the lifetime duration of smoking was also found to be a determinant of initial plasma fibrinogen levels (Meade et al 1987). Fibrinogen levels begin to fall soon after smoking is discontinued but it can take 5 to 10 years before they returned to the levels found in life-long non-smokers (Lee et al 1990, Meade et al 1987). A switch from cigarette to cigar smoking is inexplicably associated with a large increase in plasma fibrinogen (Meade et al 1987), which is in keeping with the observation that cigar smokers remain at an increased risk of ischaemic heart disease (Kaufman et al 1987). There is evidence that part of the relationship between smoking and ischaemic heart disease may be mediated through plasma 
fibrinogen (Lee et al 1990, Yarnell et al 1991), although the precise mechanism for this is unclear. Further evidence for the association between smoking, fibrinogen and ischaemic heart disease is shown by the correlation of plasma fibrinogen levels with smoking habit in patients with acute myocardial infarction (before thrombolytic therapy) (Pollak et al 1991).

The consistent observation of higher plasma fibrinogen levels in heavy smokers, independent of age, may be explained by two alternative hypotheses: (i) smoking may lead to endothelial damage (resulting in activation of the coagulation system and increased production and release of clotting factors); or (ii) smoking may directly lead to increase of clotting factors (resulting in a tendency to thrombosis and the formation of mural microthrombi on an otherwise damaged endothelium). In fact, there is evidence for both of these hypotheses. For example, cigarette smoking has been shown to cause endothelial damage and platelet activation, and post-mortem studies have demonstrated accelerated atherogenesis in the coronary arteries of young smokers (FitzGerald et al 1988). Another possible mechanism of the effects of smoking appears to be the activation of lung macrophages, which can produce hepatocyte stimulating factor, increasing the liver synthesis of fibrinogen (Ritchie et al 1982).

\section{Miscellaneous conditions}

Control of plasma fibrinogen levels is complex, as there are also associations of plasma fibrinogen not only with environmental factors (such as alcohol intake, smoking, exercise, social class, age, obesity) or the acute phase response, but also with genetic factors (Green and Humphries 1989).

Exercise Strenuous exercise is associated with lower plasma fibrinogen (and cholesterol) concentrations, equivalent to a difference of $15 \%$ in the risk of ischaemic heart disease, when compared to a sedentary lifestyle (Connelly et al 1992). Similar changes of plasma fibrinogen with exercise were seen in the Caerphilly Prospective Heart Study, the Scottish Health Heart Study and the Gothenburg study (Elwood et al 1993, Lee et al 1990, Rosengen et al 1990). 
Alcohol

Increased alcohol consumption may have a small but significant effect on decreasing plasma fibrinogen (Lee et al 1990, Meade et al 1987). A decrease of approximately $0.78 \%$ per $10 \mathrm{~g}$ of alcohol consumed has been noted (Meade et al 1987) and is consistent with the possibility that a moderate level of alcohol consumption confers some protection against ischaemic heart disease (Moore and Pearson 1986).

Social Class Psychosocial factors have important influences on cardiovascular mortality and morbidity, and plasma fibrinogen may be partly responsible (Rosengren et al 1990). The role of social class and psychosocial factors in determining plasma fibrinogen levels however remains controversial. The Northwick Park Heart Study, for example, demonstrated a greater increase in plasma fibrinogen in those of lower social classes with an additional increased risk of a cardiac event of $5 \%$ within the next 5 years in men from social classes IV and $V$ (Meade et al 1987). Similar findings of a higher plasma fibrinogen in lower social classes were also demonstrated in a cross-sectional Danish population study (Møller and Kristensen 1991) and in the Scottish Health Heart Study (Lee et al 1990). In contrast, however, the Gothenburg study found no independent association between fibrinogen concentration and occupational class (Rosengren et al 1990).

Hyperlipidaemia Plasma fibrinogen is also increased in patients with hyperlipidaemia (Lowe et al 1979, Simpson et al 1983, Stone and Thorp 1985). The interaction between plasma fibrinogen and cholesterol levels is demonstrated in the Leigh Study, where the incidence of heart attack was six times greater in those with high plasma fibrinogen ( $\geq 3.5 \mathrm{~g} / \mathrm{l})$ and cholesterol levels $(\geq 6.2 \mathrm{mmol} / \mathrm{l})$ when compared to those with low fibrinogen levels (Stone and Thorp 1985). Patients with Type II hyperlipoproteinaemia also have increased levels of plasma fibrinogen and platelet aggregates, with the normal rise in fibrinogen during adult life being abolished by a premature increase (Lowe et al 1979). This finding is consistent with an increased risk of premature atherosclerosis and thrombotic complications in these patients. In the Copenhagen study discussed above, plasma fibrinogen was closely 
associated with LDL cholesterol and HDL cholesterol levels (Møller and Kristensen 1991). One Swedish study (Korsan-Bengtsen et al 1972) and the Scottish Heart Health Study (Lee et al 1990) also showed a correlation between plasma fibrinogen and total cholesterol levels.

Patients with hypertriglyceridaemia (Type IV hyperlipidaemia) also have significantly higher concentrations of plasma fibrinogen when compared to controls, with an associated lowering of fibrinolytic activity (Simpson et al 1983). In contrast, one crosssectional study demonstrated a negative correlation between plasma fibrinogen and triglyceride levels (Korsan-Bengtsen et al 1972). These inconsistent findings, therefore, mean that the relationship between fibrinogen and triglycerides is not yet established, although alcohol may be a confounding factor (as moderate alcohol consumption may increase triglycerides but reduce plasma fibrinogen levels).

The treatment of hyperlipidaemia may alter the hypercoagulable state seen in these patients. For example, patients with Type IV hyperlipidaemia treated with diet alone (or with diet and clofibrate) showed no change in plasma fibrinogen levels, although triglycerides and some clotting factors (VIIC and Xc) were lowered (Simpson et al 1983). In contrast, the large (15000 men) WHO Co-operative trial of clofibrate (a drug which lowers cholesterol and triglycerides) showed a reduction in plasma fibrinogen levels (Green et al 1989).

Valve surgery Valve surgery is a risk factor for increased thromboembolic risk and this has been investigated in one small study, where plasma fibrinogen, plasma viscosity, red cell aggregation and spontaneous platelet aggregation were all found to be increased in patients after both mechanical and bioprosthesis heart valve replacements when compared to normal subjects (Koppensteiner et al 1991).

\section{Dental disease}

Dental disease is associated with myocardial infarction, and increased plasma fibrinogen and white cell counts may partly explain this (Kweider et al 1993). For example, chronic inflammatory gingival and periodontol infection, which is common in populations with high rates of coronary artery disease (such as Glasgow), may increase plasma fibrinogen and white cell count levels (Kweider et al 1993). 
Genetic factors

There is also a significant genetic influence on plasma fibrinogen formation and genetic heritability may account for up to $51 \%$ of the variance of the plasma fibrinogen level (Hamsten et al 1987). The development of DNA typing may therefore identify genotypes at high risk of ischaemic heart disease (Humphries et al 1987). For example, the mean fibrinogen level in individuals with the genotype B1B2 is $2.98 \mathrm{~g} / \mathrm{l}$ which is similar to the levels found in those free from heart disease in the Northwick Park heart study (Humphries et al 1987, Meade et al 1986). However, individuals with the genotype B2B2 have a mean level of $3.7 \mathrm{~g} / 1$ which would potentially increase the risk of ischaemic heart disease by $75 \%$ (Humphries et al 1987, Meade et al 1986). Certain fibrinogen genotypes have also been associated with an increased risk of peripheral atherosclerosis, although the influence was not mediated simply by way of increased fibrinogen concentrations (Fowkes et al 1992). A prospective study of the predictive value of 'high fibrinogen' genotypes for cardiovascular disease is therefore needed.

\section{Regional variations in plasma fibrinogen levels}

A wide range of reference values for plasma fibrinogen appears to exist with a mean 'normal' value between 2.3 to $3.1 \mathrm{~g} / /$ in different population studies (Ernst 1991). This is independent of patient characteristics such as age and sex, and an unidentified environmental factor may well be the cause. However, these differences may also be methodological (Machin and Mackie 1993), hence an international standardisation is also required so that plasma fibrinogen values can become directly comparable between studies.

\section{Measures reducing plasma fibrinogen}

Preventive measures against cardiovascular disease risk from high plasma fibrinogen levels require the diagnostic determination of fibrinogen concentrations, with the aim of identifying and optimally treating high risk patients. General measures such as stopping smoking and increasing physical exercise should be attempted. Therapeutic strategies may 
potentially be developed to specifically lower plasma fibrinogen in the same way as cholesterol lowering drugs do today.

Several drugs have been reported to lower fibrinogen concentrations, including ticlopidine, stanzolol, oxypentifylline, calcium dobesilate, propanolol, nislodipine and the fibrates (Ernst 1991). However these drugs have pharmacological effects other than lowering fibrinogen concentrations and are not practical therapeutic options.

The role of diet in reducing plasma fibrinogen remains controversial. For example, fish oil supplementation (rich in omega-3 fatty acids) may result in reduction in plasma fibrinogen levels (Hostmark et al 1988, Radack et al 1989). In contrast, the Northwick Park study, however, did not show any significant effect of dietary changes (Meade et al 1986). Moderate alcohol consumption, increased consumption of garlic, regular exercise, weight loss and better diabetic control are also reported to have favourable fibrinogen lowering effects (Ernst 1991, Machin and Mackie 1993).

\section{Conclusion}

Despite the intense interest in plasma fibrinogen as a cardiovascular risk factor, there is little or no information on the role of plasma fibrinogen in contributing to a prothrombotic state in conditions (and procedures) associated with an altered cardiac thromboembolic risk, for example, in patents with chronic and paroxysmal atrial fibrillation, the cardioversion of atrial fibrillation to sinus rhythm, the initiation of anticoagulation therapy and patients with left ventricular aneurysms. These areas will be investigated in this thesis.

\section{FIBRIN D-DIMER FRAGMENT.}

\section{Introduction}

Fibrin degradation products from crosslinked fibrin (XL- FDP) contain the Ddimer fragment, which is produced by fibrinolytic breakdown of insoluble clot (formed by cross-linked fibrin monomers). Measurement of this plasma factor reflects intravascular levels of fibrin turnover, without interference from fibrinogen or non-cross linked fibrin. Plasma Ddimer levels are therefore a potential indirect marker of intravascular thrombus formation, 
reflecting a hypercoagulable intravascular state. However, there is little or no information on the clinical value of plasma fibrin D-dimer as a marker of a prothrombotic state.

\section{Fibrin D-dimer and thrombogenesis}

Elevated levels of D-dimer are found in conditions associated with intravascular (and possibly extravascular) activation of the coagulation system, including those with a predisposition to venous thrombosis (Declerck et al 1987). Abnormal levels of fibrin Ddimer in plasma have been found in patients with disseminated intravascular coagulation, pulmonary thromboembolism and deep venous thrombosis (Harrison et al 1993, Knecht et al 1992). It is known, however, that the deposition of fibrin in thrombus formation is a function of the surface area of the clot and of the activity of the thrombotic factors associated with the clot. Hence, even the formation of very small clots can result in active fibrin deposition and the production of D-dimer (and other factors such as fibrinopeptide A and fibrinopeptide BB1542, which are byproducts of the coagulation cascade) (Declerck et al 1987). This suggests that plasma fibrin D-dimer is a sensitive indicator of even small amounts of thrombus although its clinical value remains to be demonstrated.

Measurement of plasma D-dimer concentration may therefore be a useful screening method for patients at risk of mobile or 'active' thrombi. This was demonstrated in a prospective study of 63 patients with mitral stenosis in which a significantly elevated plasma Ddimer level was found in $\mathbf{1 0}$ patients having mobile intracardiac thrombus when compared to patients with a non-mobile thrombus (Yasaka et al 1991). Levels are also increased in patients with vascular disease (Fowkes et al 1993), with histopathological evidence for high amounts of fibrinogen and fibrin D-dimer in aortic thrombi, atherosclerosis plaques and early gelatinous arterial lesions (Bini and Kudryk 1992, Smith et al 1990).

Elevated fibrin D-dimer levels have been found in patients with peripheral vascular disease (Al-Zahrani et al 1992, Fowkes et al 1993) and this measurement has been shown to have prognostic implications in these patients. In a study of 617 patients with peripheral vascular disease, baseline fibrin D-dimer levels were closely related to future coronary events (both fatal and non-fatal, with a relative risk of 4.4 between upper and lower 
quintiles) and progression of peripheral vascular disease (Fowkes et al 1993). This study therefore suggests that fibrin formation contributes to the progression of coronary and peripheral atherosclerosis, and is consistent with the hypothesis that fibrin D-dimer may be a useful index of intravascular fibrin turnover and the contribution of thrombosis to arterial disease.

Plasma fibrin D-dimer may therefore prove useful in assessing the thrombogenic risk for patients with conditions that predispose to thromboembolism, for example, chronic atrial fibrillation. For example, two recent studies have suggested that plasma levels of D-dimer are increased in patients with atrial fibrillation, whether or not structural heart disease is present, when compared to controls in sinus rhythm (Gustafsson et al 1990, Kumagai et al 1990). However, these studies were small and the controls were not population-based. The usefulness of plasma D-dimer as a marker of thrombogenesis in other conditions at risk of thromboembolism, such as left ventricular aneurysms, paroxysmal atrial fibrillation, and in conditions that alter thromboembolic risk, such as anticoagulation and cardioversion from atrial fibrillation to sinus rhythm, have not been previously investigated and these areas will be addressed in this thesis.

\section{VON WILLEBRAND FACTOR}

Introduction

The vascular endothelium is intimately associated with the regulation of vessel tone and permeability, haemostasis, fibrinolysis and synthesis of growth factors. Von Willebrand factor (WWF) is one such factor, which is synthesised by and stored in endothelial cells (and also megakaryocytes and platelets) and when released appears to mediate platelet aggregation and adhesion to the vascular endothelium (Badimon et al 1992, Jansson et al 1991).

Abnormalities in the blood vessel wall (including endothelial dysfunction) may result in an increased propensity towards thrombus formation (Virchow's triad). As VWF is released when endothelial cells are damaged, VWF and other fibrinolytic variables have been used as indicators of endothelial dysfunction (Boneu et al 1975). Plasma concentrations of 
VWF are increased therefore in disorders that affect the cardiovascular system, for example, ischaemic heart disease (Jansson et al 1991), pregnancy-induced hypertension (Brenner et al 1989), diabetes mellitus (Greaves et al 1987), cerebrovascular disease (Mettinger 1982, Wahlberg et al 1980) and deep venous thrombosis (Wahlberg et al 1980).

\section{Cardiac disease}

There is an association between vWF levels and ischaemic heart disease. For example, elevated concentrations of VWF are found in patients with previous myocardial infarction (Hamsten 1986, Schmitz-Huebner et al 1988) and also appear to be an index of increased risk for reinfarction and mortality in survivors of myocardial infarction (Jansson et al 1991). There is also an association with the clinical severity of angina (Moisseev 1988), indicating a role for endothelial dysfunction in the pathogenesis of coronary artery disease.

\section{Thromboembolic events}

High concentrations of vWF have been associated with an increased incidence of thromboembolic events. For example, there is an association with ischaemic cerebrovascular disease (Mettinger 1982, Uchiyama et al 1983, Wahlberg et al 1980) and deep venous thrombosis (Wahlberg et al 1980). These findings suggest that a high concentration of vWF may be an index of atherosclerosis and/or thrombosis.

\section{Pulmonary vascular disease}

The pulmonary vasculature also appears to have significant influences on plasma vWF levels. For example, in primary pulmonary hypertension, pulmonary endothelial cells show evidence of injury, and elevations of plasma vWF have been demonstrated in this condition, which may contribute to the risk of thrombosis (Geggel 1987). Elevated plasma VWF has also been demonstrated in patients with elevated pulmonary vascular resistance and decreased cardiac output, irrespective of the presence of mitral stenosis (Penny et al 1991). These findings are in keeping with a haemodynamically-induced increase in the endothelial release of vWF. 
Diabetes

Abnormalities of WWF have also been demonstrated in diabetics (Collier et al 1992, Greaves et al 1987, Stehouwer et al 1992). For example, a raised urinary albumin excretion in non insulin dependent diabetes is associated with an increased risk of cardiovascular disease (Stehouwer et al 1992). Endothelial dysfunction is thought to a possible explanation for this association and the marker for this, plasma vWF, is found to be elevated in such patients (Collier et al 1992, Stehouwer et al 1992). It is therefore considered that dysfunction of vascular endothelium may be one link between the microalbuminuria and atherosclerotic cardiovascular disease found in non-insulin dependant diabetics.

\section{Conclusion}

Von Willebrand factor may be a good marker for endothelial dysfunction in a variety of disease states, with a significant potential contribution towards thrombogenesis via platelet adhesion and aggregation. There is little information, however, on its relationship to other prothrombotic markers, such as fibrinogen, D-dimer or lipoprotein (a). This aspect will be further studied in patients with chronic atrial fibrillation (chapter 3) and left ventricular dysfunction (chapters 8 and 9 ).

\section{LIPOPROTEIN (a)}

Introduction

Lipoprotein (a) [Lp (a)] has been recognised for nearly 30 years as a partly genetically-determined lipoprotein associated with an increase in atherogenesis and thrombogenesis (Rees 1991, Scott 1991). For example, elevated Lp (a) levels are associated with an increased risk for coronary artery disease, stroke, restenosis after coronary artery bypass surgery and thoracic aortic atherosclerosis (Hoff et al 1988, Nishino et al 1993, Shintani et al 1993, Woo et al 1991). Lp(a) has therefore stimulated great interest as yet another risk factor for cardiovascular and cerebrovascular disease. 
Despite intense interest in this enigmatic lipoprotein its function (and factors that influence its levels) remains largely unknown. It has been suggested, for example, that apolipoprotein (a) [a molecule contained within lipoprotein (a)] could offer some selective advantage in wound or tissue repair, as it may help deliver cholesterol by attaching (by its fibrin-binding capability) to thrombus (Lawn 1992, Scanu 1992). The importance of Lp(a) phenotypes in influencing the pathogenic effects of $L p(a)$ (Utermann et al 1988) and the significance of the associated homology of a molecule unique to $\operatorname{Lp}(\mathrm{a})$, apolipoprotein (a), with plasma plasminogen (which may reduce fibrinolysis) remain unanswered controversies (Scott 1991). In addition, the contribution of lipoprotein (a) to the mechanisms of thrombogenesis (for example, clotting factor abnormalities, fibrin turnover, endothelial dysfunction) has not been clearly defined.

\section{Biochemistry and pathophysiology}

$L p(a)$ is a variant of low density lipoprotein (LDL). Similarly to $L D L$, the main protein component of $L p(a)$ is apolipoprotein B (apo B), but in addition, there is also another large disulphide-linked glycoprotein molecule, apolipoprotein (a) (apo(a)) present. Genetic variation of this molecule [apo(a)] is the primary determinant of $L p(a)$ phenotypes. Size isoforms of apo(a) are inherited as Mendelian codominant traits and are associated with variations in the plasma concentrations of $L p(a)$, and thus with the various degrees of risk for coronary artery disease (Seed et al 1990, Utermann et al 1988).

Apo(a) is homologous with part of the plasminogen molecule, from which the enzyme plasmin is released by tissue plasminogen activator during the process of fibrinolysis. Indeed plasminogen and apo(a) are encoded by genes which both map to chromosome 6q26-27 (Drayna et al 1988). This relationship is important as apo(a) size isoforms and plasma $L p(a)$ concentrations are each determined by genetic variation at the apo(a) locus (Drayna et al 1988). In a study of 473 patients, for example, apo(a) glycoprotein polymorphism accounted for $41.9 \%$ and $9.6 \%$ of the variability in $L p(a)$ and total cholesterol levels respectively, which represents the strongest effect of a single polymorphic gene on plasma lipid and lipoprotein levels reported so far (Boerwinkle et al 1989). Further evidence for a genetic influence on 
$L p(a)$ levels was demonstrated in the Bogalusa Heart Study, a study of 2438 children (aged 817) from a biracial community (Srinivasan et al 1991). $L p(a)$ levels were 1.7-fold higher in blacks than whites, and in white children the prevalence of parental myocardial infarction was higher in those with elevated levels of $L p(a)$; however, the latter observation was not seen in black children (Srinivasan et al 1991).

Experiments in vitro suggest that apo(a) interferes with uptake and activation of plasminogen by receptors on cell surfaces and fibrin (Gonzalez-Gronow et al 1989, Miles et al 1989). Plasminogen receptors, which are widely distributed on endothelial cells and blood cells, promote thrombolysis by accelerating plasminogen activation and protecting plasmin from inhibition. If, by molecular mimicry, $L p(a)$ competes with plasminogen for receptors then the binding of plasminogen to endothelial cells and thrombolysis would be inhibited and thrombosis promoted (Gonzalez-Gronow et al 1989, Miles et al 1989). Experimental evidence for this comes from the demonstration that high Lp(a) levels impair the binding of plasminogen to fibrin, thus reducing the generation of plasmin by fibrin-bound tissue-type plasminogen activator (Rouy et al 1991). Lp(a) also appears to regulate the synthesis by the endothelium of a major protein involved in the fibrinolytic system, plasminogen activator inhibitor-1 (PAI-1): $L p(a)$ may therefore promote a prothrombotic state by increasing in PAI-1 activity, thus interfering with endothelial cell and circulating plasmin generation (Etingin et al 1991).

$\mathrm{Lp}(\mathrm{a})$ is also associated with atherogenesis (Figure 1.1), and an interaction with fibrinogen (and other coagulation factors) may therefore exist. The accumulation of Lp(a) at sites of vascular injury, in association with impairment of fibrinolysis, may be important factors in the development of atherosclerosis and associated thrombosis (Rouy et al 1991). The mechanism for this detrimental effect of $L p(a)$ may relate to the macrophages that are also attracted to sites of vascular injury. When exposed to excessive $L p(a)$ and oxidised low density lipoprotein (LDL), these macrophages become foam cells, the precursors of the atherosclerotic plaque (Scanu 1992). 


\section{Epidemiological studies}

Until now, few epidemiological studies have investigated the significance of this lipoprotein. Recently, the Framingham Offspring Study measured serum $L p(a)$ levels in 1284 men and 1394 women free of cardiovascular disease (Jenner et al 1993). There was an inverse association between plasma $L p(a)$ and triglyceride levels, but this relationship was weak as triglycerides only accounted for $0.5 \%$ of the variation in lipoprotein (a) levels. There was no significant association of lipoprotein (a) levels with total and LDL cholesterol levels, body mass index, alcohol consumption and with age (although there was a trend towards higher levels in postmenopausal women) (Jenner et al 1993). In addition, healthy subjects in the Prospective Cardiovascular Munster (PROCAM) study demonstrated a weak relationship between plasma fibrinogen and plasma fibrinogen in both sexes, but not with D-dimer or other indicators of fibrinolysis (for example, tissue-type plasminogen activator) (Heinrich et al 1991a). From this study, it appears that very few factors may influence plasma $L p(a)$ levels, which tend to remain remarkably constant within an individual (Rees 1991).

\section{Ischaemic heart disease}

Patients with coronary artery disease have higher $L p(a)$ levels than controls (Farrer et al 1993, Genest et al 1991, Genest et al 1992, Sandholzer et al 1992). In a prevalence study of 180 patients aged $<60$ years, this finding was also independent of conventional risk factors such as smoking, hypertension, diabetes, LDL and HDL cholesterol or apolipoprotein A-1 and B levels (Genest et al 1991). One recent study also showed that $L p(a)$ was not responsible for the risk association between impaired glucose tolerance and coronary artery disease (Farrer et al 1993). Family studies by Genest et al (1991) demonstrated a large genetic influence and little environmental influence on $L p(a)$ levels.

Coronary heart disease risk also correlates directly with plasma concentrations of $L p$ (a) (Rosengren et al 1990). The Gothenburg study, for example, was a prospective case-control study with a six-year follow up period, which found that men who had suffered a coronary event had significantly higher serum $L p(a)$ concentrations than controls, independent of other risk factors (Rosengren et al 1990). Additionally, in the PROCAM study, 
young (aged less than 46 years) male survivors of myocardial infarction showed that increased $\mathrm{L} p$ (a) concentrations constituted an independent risk factor for early myocardial infarction (Sandkamp et al 1990). Concentrations of $L p(a)$ did not, however, correlate with age or other well-known risk factors for early myocardial infarction such as apolipoproteins Al and B, LDL cholesterol and HDL cholesterol (Sandkamp et al 1990). In addition, this study showed an association with fibrinogen concentration but not with euglobulin fibrinolysis activity and other fibrinolytic parameters (Sandkamp et al 1990).

An increased $L P(a)$ level also has implications for patients post-coronary artery bypass surgery. For example, a large angiographic study suggested that increased Lp(a) levels were associated with the degree of saphenous vein bypass graft restenosis, with a stepwise correlation of mean $L p(a)$ levels to increasing graft stenosis (Hoff et al 1988).

The large (4081 subjects) Helsinki Heart Study, in contrast, did not demonstrate that serum $L p(a)$ level was predictive of future coronary events, despite 5-years follow-up (Jauhiainen et al 1991). This is supported by an angiographic study of 56 men which showed that serum $L p(a)$ measurement did not discriminate between patients with and without progression of angiographic coronary artery lesions (Perski et al 1992). These two studies are therefore at variance with evidence discussed earlier.

Phenotypes of the apo(a) component of $L p(a)$ may explain the considerable variation between individuals with familial hypercholesterolaemia in susceptibility to coronary heart disease (Sandholzer et al 1992). In patients with familial hypercholesterolaemia, the frequencies of the apo(a) phenotypes and alleles differed significantly between patients with and without coronary heart disease thus suggesting a genetic influence (Seed et al 1990). For example, the allele $L p S 2$, which is associated with high $L p(a)$ levels, was found more frequently amongst patients with coronary heart disease (Seed et al 1990). In contrast, the $L p^{S 4}$ allele, which is associated with low $L p(a)$ levels, was more frequent amongst those without coronary heart disease (Seed et al 1990). This increase in risk was independent of age, sex, smoking status and serum levels of total cholesterol, triglyceride or high density lipoprotein (HDL) cholesterol (Seed et al 1990). However, no agreement on the definite number of apo (a) alleles in the general population has been reached - reported numbers of 
apo(a) alleles or isoforms range between 11 and 24, although more may exist (Cohen et al 1993, Jenner et al 1993).

Serial determinations of $L p(a)$ levels after an acute myocardial infarction demonstrate no change in $L p(a)$ following myocardial infarction. For example, a study of 124 patients showed no difference between those with myocardial infarction, unstable angina and stable angina, between patients with myocardial infarction with or without thrombolytic treatment, nor between late (at 6 hours) and early measurements of $L p(a)$ in patients with unstable angina and myocardial infarction (Qiu et al 1989). The extent of decrease in levels of plasminogen, alpha-2 antiplasmin and fibrinogen in the patients given thrombolytic therapy did however correlate positively with higher $L p(a)$ blood levels (Qiu et al 1989). Patients with high $L p(a)$ levels also respond equally well (as reflected by angiographic reperfusion) to thrombolytic therapy as did patients with normal or low levels of $L p(a)$ when given either recombinant tissue plasminogen activator (rt-PA) or human single chain urokinase-type plasminogen activator (scu-PA) (von Hodenberg et al 1991). Similar results were also obtained in other studies using scu-PA and alteplase (rt-PA) (Armstrong et al 1990, Tranchesi et al 1990, Tranchesi et al 1991), indicating a poor relationship of pretreatment $L p(a)$ levels to angiographic recanalization. These studies therefore suggest that serum $L p(a)$ levels are not increased in acute myocardial infarction and unstable angina and are not influenced by thrombolytic therapy (Qiu et al 1989). However, in these studies (Armstrong et al 1990, von Hodenberg 1991, Qiu et al 1989, Tranchesi et al 1990), there was only a short time interval between serial measurements of $L p(a)$ and the isoforms of $L p(a)$ were not measured despite this polymorphism having an influence on total levels of $L p(a)$ and different alleles having different contributions to coronary artery disease (Qiu et al 1989, Sandholzer 1992).

In contrast, others have demonstrated an increase in $L p(a)$ following myocardial infarction, with a return to initial levels after one month (Abe et al 1989, Maeda et al 1989). Measurements of acute phase proteins also showed a rise and fall after the acute event, suggesting that $L p(a)$ may have some characteristics of an acute phase reactant, with a role in recovery from tissue damage. Another study showed no change in absolute Lp(a) values following myocardial infarction, but an increase was demonstrated if the relative change 
in $L p(a)$ values was plotted (Slunga et al 1992). This change, however, did not correlate with changes in acute-phase proteins, and individual $L p(a)$ responses were very inconsistent.

\section{Cerebrovascular disease}

The relationship between serum lipids and lipoproteins in cerebrovascular disease is not as clear-cut as in coronary artery disease. However, $L p$ (a) has been shown to be associated with ischaemic cerebrovascular disease (Shintani et al 1993, Woo et al 1991, Zenker et al 1986). Even in normolipaemic patients, increased serum lipoprotein (a) levels and intermediate density lipoprotein abnormalities (and decreased high density lipoprotein levels) are major risk factors for stroke (Pedro-Botet et al 1992, Shintani et al 1993). In addition, median $L p(a)$ levels in patients with cerebrovascular disease were similar to that found in patients with coronary artery disease (Zenker et al 1986).

These findings support the hypothesis that lipoprotein (a) is a marker for atherosclerosis and thrombogenesis. However, whether or not $L p(a)$ itself can cause atherosclerosis (or thrombosis) or is just a factor indicating an atherosclerotic or thrombogenic process is still unresolved (Woo et al 1991).

As for coronary artery disease, the genetic influence remains on the relationship between $L p(a)$ and cerebrovascular disease, which is associated with apolipoprotein E polymorphism. This polymorphism affects plasma levels of lipoprotein (a), and the presence of apolipoprotein E2 allele, for example, decreases the mean lipoprotein (a) level by $26 \%$, whilst the E4 allele increases the lipoprotein (a) level by $26 \%$ (de Knijff et al 1991). The latter allele (E4) was found more prevalent in men with ischaemic strokes, suggesting a possible role for the E4 allele as a predisposing genetic marker for stroke (Pedro-Botet et al 1992).

\section{Other conditions influencing $L p(a)$}

As discussed above, lipoprotein (a) is partially genetically-determined. The nearly constant blood levels of $L p(a)$ suggest that its gene is partially constitutive, that is, it is transcribed independently of the physiological state of the cell. This is in contrast to genes 
which are inducible (and can be 'turned on' by cellular signals) or repressible (which are normally 'on' and can be turned 'off'). However, some portions of $L p(a)$ production may be regulated.

There is evidence, for example, that $L p(a)$ levels are elevated in certain acquired conditions, such as renal disease and poorly controlled diabetic patients, whilst liver disease leads to lower concentrations. In a cross-sectional study of 93 diabetics, those with high (>8.0\%) $\mathrm{HbA}_{1 \mathrm{c}}$ (indicating poor diabetic control) had higher median levels of $\mathrm{Lp}(\mathrm{a})$ compared to controls or diabetics with $\mathrm{HbA}_{1 \mathrm{c}}<8 \%$ (Ramirez et al 1992). Similarly, elevated $L p(a)$ levels have been found in association with impaired renal function and in patients on haemodialysis (Cressman et al 1992, Parra et al 1987). These findings may contribute to the high risk of atherosclerosis observed in such patients. In a study of patients with cirrhosis, however, lower $L p(a)$ levels were seen with increasing severity of liver disease (being undetected in a high proportion of patients with the most severe disease) (Feely et al 1992).

Some conditions are also well known to contribute to changes in plasma lipoproteins although the effects on $L p(a)$ are as yet uncertain. One notable example is the menopausal status, with adverse changes in lipids and lipoproteins postmenopausally, which may contribute to the risks of coronary artery disease in these patients (Bonithon-Kopp et al 1990). A trend towards higher levels of $L p(a)$ in postmenopausal women was noted in the Framingham Offspring Study (Jenner et al 1993)

\section{Measures to reduce $L p(a)$}

It has been suggested that the following persons should have $L p(a)$ levels measured: (i) those with personal or family histories of premature cardiovascular disease (Age $\leq 55$ years); (ii) patients with cardiovascular disease but normal 'routine' lipid profile; (iii) patients with recurrent coronary artery stenosis; and (iv) family members of the above patients (Scanu 1992). Lowering raised $L p(a)$ levels however poses another problem. Modifying the diet does not appear to affect $L p(a)$ concentrations (Masarei et al 1984). In the Framingham Offspring Study, treatment with beta-blockers, cholesterol-lowering drugs, oral hypoglycaemics, insulin, thyroid replacement, diuretics and other antihypertensive 
medication did not have any significant effect on plasma Lp(a) levels (Jenner et al 1993). Serum $L p(a)$ concentrations may, however, respond to nicotinic acid (Carlson et al 1984) and bezafibrate (Bimmermann et al 1991). The use of bezafibrate in patients with high Lp(a) levels (above $30 \mathrm{mg} / \mathrm{dl}$ ), for example, may result in a significant reduction of $L p(a)$ by $39 \%$, which was independent of sex and type of hyperlipoproteinaemia (Bimmermann et al 1991). Bezafibrate may therefore be a useful drug, with effects in lowering lipids (cholesterol and triglyceride), fibrinogen and $L p(a)$ levels.

Despite the beneficial effect of bezafibrate, there was, however, no difference with the use of another fibrate, gemfibrozil (Jauhiainen et al 1991). Other potential therapeutic interventions include neomycin and niacin (reducing $L p(a)$ by almost $50 \%$ ) (Gurakar et al 1984), and anabolic steroids (Albers 1984). Extracoporeal removal by apheresis can achieve even greater reductions (Armstrong et al 1985, Rees 1991).

The influence of reducing $L p(a)$ levels on outcome in coronary artery disease and stroke, if any, is unknown. There is, to date, no published data showing any benefit in lowering $L p(a)$ concentrations in individual patients (Rees 1991).

\section{Conclusion}

The therapeutic implications of finding a raised level of $L p(a)$ depend upon the interpretation of suggestive but inconclusive data. An important question, therefore, is whether or not the increased risk of coronary artery disease associated with $L p(a)$ levels is related to other factors involved in thrombogenesis and atherogenesis (such as fibrinogen, von Willebrand factor, LDL-cholesterol, etc). In addition, if the association between $\operatorname{Lp}(\mathrm{a})$ and cardiovascular disease is causal, does the mechanism involved reflect the atherogenicity or thrombogenicity of $L p(a)$ or a combination of both? These questions may be answered by a study of $L p(a)$ levels in relation to other blood prothrombotic factors (fibrinogen, fibrin D-dimer, von Willebrand factor, etc) in conditions at high risk of thromboembolism, as is undertaken in this thesis. 


\subsection{AIMS OF STUDIES REPORTED IN THIS THESIS}

My thesis investigates the role of plasma fibrinogen (whose elevation is associated with the risk of increased thrombotic and cardiovascular events) and fibrin D-dimer (fibrin degradation products, which reflect intravascular fibrin turnover) in two common cardiovascular conditions associated with thromboembolism, that is, atrial fibrillation and left ventricular dysfunction. Endothelial dysfunction may interact with these coagulation factor abnormalities and therefore the role of von Willebrand factor (VWF), which is a marker of endothelial dysfunction with prothrombotic potential, will also be investigated. In view of the possible competitive antagonism (or inhibitory effects) of $L(a)$ on fibrinolysis $I$ also hypothesised an inverse relationship between $L p(a)$ and plasma D-dimer levels. Raised Lp(a) levels in conjunction with endothelial dysfunction (as reflected by plasma vWF levels) may contribute to the processes of thrombogenesis and atherogenesis. Measurement of these markers of thrombogenesis may therefore provide a possible approach to identify those patients who are at highest risk of thromboembolism.

Initially, I conducted a survey of emergency admissions with atrial fibrillation into a district general hospital, to determine the prevalence of the condition and to assess management strategies and whether or not risk factors were being identified. This survey also allowed identification of patients with chronic atrial fibrillation for further study, in whom markers of thrombogenesis were measured, detailed echocardiography performed and the effects of antithrombotic therapy (aspirin or warfarin) assessed. I also correlated my findings on detailed echocardiography (left atrial size and volume, left ventricular size and function, etc) with these prothrombotic markers.

The hypothesis that fibrin D-dimer is a useful marker for thrombus formation was also tested by looking at subgroups at intermediate risk of thromboembolism, such as patients with paroxysmal atrial fibrillation and valve disease, and by assessing the effects of therapy (in particular, cardioversion and anticoagulation). As cardioversion from atrial fibrillation to sinus rhythm is an additional thromboembolic risk, I therefore measured serial samples of these prothrombotic factors in patients undergoing cardioversion, to try to delineate the length of the 'risk period'. In addition, as anticoagulant therapy has been shown 
to reduce the risks of thromboembolism in patients with atrial fibrillation, I conducted serial measurements of these prothrombotic factors in order to determine the effects of introducing oral anticoagulant therapy in these patients.

Finally, as patients with left ventricular aneurysms are also at increased thromboembolic risk, I assessed the levels of these markers of thrombogenesis in patients with left ventricular aneurysms compared to patients with varying degrees of left ventricular dysfunction (other than aneurysm formation) and to patients with normal left ventricular function. I assessed left ventricular function by performing (and interpreting) detailed echocardiography and $99 \mathrm{~m}$ Technetium isotope radionuclide ventriculograms, and determining the relative contributions of systolic and diastolic dysfunction to thromboembolic risk in patients with coronary artery disease. The effects of introducing oral anticoagulant therapy were also determined in these patients. I hypothesised that impaired ventricular contractility (systolic dysfunction) and impaired ventricular relaxation (diastolic dysfunction) might have different haemorheological effects, resulting in different contributions to thrombogenesis.

\section{Specific hypotheses to be investigated:}

1. That plasma levels of fibrinogen, D-dimer, von Willebrand factor and lipoprotein (a) may be elevated in patients with cardiac disorders which predispose to systemic thromboembolism (such as sustained/paroxysmal atrial fibrillation, left ventricular aneurysm / dysfunction), suggesting possible roles for fibrinogen, increased fibrin formation, endothelial dysfunction, decreased fibrinolysis and rheological disturbance in thrombogenesis.

2. That levels of these markers may be related to size of cardiac chambers and other structural abnormalities, and to the presence of ventricular dysfunction.

3. That therapeutic interventions which alter thromboembolic risk (for example, cardioversion, warfarin, aspirin) may alter levels of these markers in the appropriate direction. 
4. That there is an inverse relationship between plasma lipoprotein (a) $(L p(a))$ and plasma $D$ dimer levels, in view of the possible competitive antagonism (or inhibitory effects) of $L p(a)$ on fibrinolysis.

The results will be critically discussed, as will be the possible roles of these markers in the risk assessment and pathogenesis of cardiac thromboembolism. 
CHAPTER 2

\section{A SURVEY OF ATRIAL FIBRILLATION}




\subsection{AN OVERVIEW OF ATRIAL FIBRILLATION}

\section{Introduction}

Atrial fibrillation (AF) is the commonest sustained cardiac rhythm disorder. It is also a clinically important condition, with significant prognostic and therapeutic implications. Atrial fibrillation is particularly prevalent among the elderly, ranging from $0.5 \%$ at $50-59$ years to 8.8\% at 80-89 years (Kannel et al 1982, Wolf et al 1991), and can be either chronic or paroxysmal. The presence of atrial fibrillation confers a significant increase in overall morbidity and mortality (Kannel et al 1982, Repique et al 1992). Despite this, atrial fibrillation is sometimes regarded as a relatively trivial disorder, although many patients with chronic atrial fibrillation require long term treatment with potent antiarrhythmic and anticoagulant drugs, which can have important drug interactions and adverse effects.

Atrial fibrillation is encountered in a wide variety of clinical settings. It may, for example, be discovered incidentally in an asymptomatic patient, it may occur in a patient with lobar pneumonia, or it may be found in a patient with a ventricular rate of 200 beats per minute who is suddenly too lightheaded to stand! The commonest aetiological factors for atrial fibrillation remain ischaemic heart disease, hypertension, thyroid disorders and rheumatic valve disease (although the incidence of the latter is decreasing in the Western world) (Alpert et al 1988). In the Framingham Study, for example, hypertension, cardiac failure and rheumatic heart disease were the commonest precursors of atrial fibrillation (Kannel et al 1982). Thyrotoxic atrial fibrillation may also be an underdiagnosed condition, as appropriate thyroid function tests are not often performed and hyperthyroidism (affecting about $1 \%$ of the population) may co-exist with ischaemic and rheumatic heart disease (Forfar et al 1979, Forfar and Toft 1982). Other noncardiac causes of atrial fibrillation are also frequently encountered, and can include pneumonia, pulmonary thromboembolism and excessive alcohol consumption (Repique et al 1992).

There remains, however, a subgroup of patients with atrial fibrillation who have no predisposing factor, which is classified as 'lone' or 'idiopathic' atrial fibrillation (Brand et al 1985). This is a diagnosis of exclusion, and has been established in $2.7 \%$ to $11.4 \%$ of all 
patients with atrial fibrillation (Repique et al 1992). Epidemiological evidence suggests however that up to one third of patients with atrial fibrillation may have idiopathic atrial fibrillation, with different clinical manifestations - the paroxysmal form (characterised by longlasting episodes), the transient recurrent form (characterised by frequent self-terminating episodes) or less commonly, the chronic form (Capucci and Boriani 1991, Houghton et al 1990). These patients (especially those aged $<60$ ) are generally accepted to be at a low thromboembolic risk with no requirement for antithrombotic therapy (Davidson et al 1989, Kopecky et al 1987). Data from the Framingham study (with an older study population; mean age 64 years), however, does suggest a fivefold increase in the incidence of stroke $(28.2 \%$ vs $6.8 \%$ in matched controls) for patients with lone atrial fibrillation (Brand et al 1985). Despite this, left atrial enlargement of $>4.0 \mathrm{~cm}$ is rarely found, and cardiac failure is unusual in such patients (Davidson et al 1989, Repique et al 1992).

The sudden onset of fast atrial fibrillation may precipitate overt heart failure, particularly if left ventricular function is already compromised by co-existing heart disease, for example, valvular or ischaemic heart disease. Less dramatic presentations of atrial fibrillation include palpitations, dyspnoea, angina or general fatigue or lethargy. Symptoms may be more marked on exercise, with a significant limitation of exercise tolerance. More important, however, is the finding that nonrheumatic atrial fibrillation increases the risk of stroke by a factor of five and is present in about $15 \%$ of patients with acute stroke (Brand et al 1985, Wolf et al 1978, Wolf et al 1991). The risk of stroke in someone with atrial fibrillation is about $5 \%$ a year and epidemiological evidence suggests that this risk increases with age, blood pressure, and other evidence of heart disease (Lowe 1992). The presence of atrial fibrillation may also increase the risk of recurrent stroke (Flegel and Hanley 1989, Sage and Van Uitert 1983) but this has not been confirmed in two other community studies (Wolf et al 1983, Sandercock et al 1992). However, in the Oxfordshire Community Stroke Project, the $17 \%$ of patients with acute stroke and atrial fibrillation had a significantly higher $\mathbf{3 0}$ day mortality than patients in sinus rhythm ( $23 \%$ vs $8 \%$ ) (Sandercock et al 1992$)$. This greater mortality is partly explained by the association of atrial fibrillation with large, total anterior cerebral infarcts, probably due to occlusion of the middle cerebral artery (Sandercock et al 1992). Similar mortality findings were 
found in a study of acute stroke patients where the $25 \%$ with atrial fibrillation had a significantly higher hospital mortality than patients in sinus rhythm (67\% vs $44 \%$ ) (Lowe et al 1983). The presence of atrial fibrillation as an adverse feature in elderly stroke patients was confirmed in the Waikato Stroke Registry (mean age 75.2 years), where those with atrial fibrillation were older, had more severe stroke deficits and a greater mortality, when compared to patients in sinus rhythm (Friedman 1991).

What, therefore, are the therapeutic benefits from treating atrial fibrillation? The major reasons for treating atrial fibrillation are as follows: to relieve symptoms of congestive heart failure, hypotension or angina that can be directly attributed to a rapid heart rate; to improve overall cardiac function; to improve exercise tolerance; and to reduce the risk of thromboembolism and stroke. The main reasons are therefore to ameliorate the adverse haemodynamic effects and the thromboembolic risks of atrial fibrillation.

Cardioversion to, and maintenance of, normal sinus rhythm remains the optimal strategy to enhance cardiac performance and reduce thromboembolic risk. The cardioversion of atrial fibrillation to normal sinus rhythm is playing an increasing role in the management of atrial fibrillation, and this (and its thromboembolic risk) is examined in chapter 4 of this thesis. As there is now evidence that cardiac function and exercise tolerance may even improve following cardioversion to sinus rhythm, this therapeutic option is increasingly used. However, there is often variation in the use of this option and the use of anticoagulant therapy before and after cardioversion. This subject is further reviewed and investigated in chapter 4 . The presence of impaired cardiac function may also contribute to an increased risk for thromboembolism and is further examined in chapters 8 and 9 of this thesis.

The role of anticoagulant therapy as prophylaxis against thromboembolism in atrial fibrillation has attracted much interest recently. There are now five large prospective randomised controlled trials on the use of oral anticoagulant therapy in the primary prevention of stroke in nonrheumatic atrial fibrillation (Boston Area Anticoagulation Trial for Atrial Fibrillation Investigators 1990, Connolly et al 1991, Ezekowitz et al 1992, Petersen et al 1989, Stroke Prevention in Atrial Fibrillation Study Group Investigators 1990, Stroke Prevention in Atrial Fibrillation Investigators 1991). Their results consistently show that moderate 
anticoagulation reduces the risk of strokes by about two-thirds without a significant rise in adverse effects. These studies have therefore established the role for oral anticoagulant therapy in atrial fibrillation. However, there continues to be some reluctance amongst physicians to introduce anticoagulant therapy, with a significant proportion of patients with atrial fibrillation still not on anticoagulant therapy in spite of there being no contra-indications (Bath et al 1993). This aspect is further investigated in this chapter and the role of anticoagulant therapy is reviewed and further studied in chapter 10 of this thesis.

Atrial fibrillation is therefore a common arrhythmia with different aetiologies, clinical presentations and therapeutic options. This opens it to wide variations in management strategies. Ideally, the management of atrial fibrillation should follow three phases: a search for an underlying cause (with appropriate investigations); control of the arrhythmia and reduction of thromboembolic risk; and finally, consideration of cardioversion to sinus rhythm. However, atrial fibrillation is frequently not managed appropriately. As atrial fibrillation is common among emergency medical admissions, I decided to conduct a prospective survey of admissions with atrial fibrillation to a district general hospital. 


\subsection{ATRIAL FIBRILLATION IN A DISTRICT GENERAL HOSPITAL}

\section{Introduction}

Atrial fibrillation presents a number of different investigation and management dilemmas. It is a common presenting arrhythmia amongst acute medical admissions to district general hospitals in the United Kingdom. However, there is little published information on the proportion of emergency admissions whom have atrial fibrillation, the admission rate relative to the population served, therapy prior to admission, therapy initiated following admission, investigations undertaken or the outcome. Additionally there appears to be a wide range of views on the optimal management of this arrhythmia amongst general physicians. This is not surprising as the management of the patient with atrial fibrillation is complicated by multiple causes, diverse therapeutic options, concerns about anticoagulation, and the proarrhythmic effects of antiarrhythmic therapy.

In view of this, I decided to survey the practice of investigation and management of this disorder in patients admitted as emergencies, amongst both general physicians and cardiologists, to Stobhill General Hospital. This is a 990-bed district general hospital with acute medical and surgical facilities, serving the North-East of the City of Glasgow and the borough of Strathkelvin. This survey also allowed me to appreciate the prevalence of the condition, the frequency of complications in my hospital, and to identify patients with established atrial fibrillation and those with paroxysmal atrial fibrillation for subsequent studies in this thesis.

\section{Materials and methods}

Stobhill General Hospital has a Coronary Care Unit, six medical wards, a Renal ward and a large Geriatric Department. There are 9 general physicians, one of whom has a major interest in Cardiology. In addition there is a one Cardiologist, two Renal physicians and 4 physicians with an interest in Geriatric Medicine, none of whom undertake general medical receiving duties. The physicians were informed at the onset of the study that an audit was going to take place as this is part of hospital protocol. Thereafter, the information was 
collected from the case records and no further discussions were held with the physicians involved.

The total number of emergency admissions to the adult medical and geriatric services during the six-month period from 1st September 1991 to 28th February 1992 was obtained from hospital records. Patients were prospectively included in this study if they were found to be in established atrial fibrillation on the admission electrocardiograph (ECG), or had developed atrial fibrillation within $\mathbf{4 8}$ hours of admission. Patients were studied from the three medical units within the hospital, the coronary care unit, renal unit and the geriatric wards; and were identified by discussion with the on-call medical or geriatric teams or by visiting the admitting ward(s) and reviewing the admission notes and ECG.

The mode of presentation, cardiac investigations carried out, the aetiology of atrial fibrillation, the treatment prior to admission and that initiated following admission, and the inpatient mortality were also noted. The survey therefore assessed the characteristics of the inpatients admitted with atrial fibrillation, length of stay, whether the aetiology of atrial fibrillation was been assessed, what investigations were undertaken (and what their impact on management was) and also actual management undertaken on the patient (at the time of atrial fibrillation, throughout their stay in hospital and at the time of discharge). An attempt was also made to determine whether or not atrial fibrillation was new at the time of admission or was a more chronic problem.

Statistical correlations were performed using Spearman's Rank Correlation method and a probability of $\leq 0.05$ was considered statistically significant.

Results

During the six months of the study the total number of emergency admissions to the medical and geriatric services was 2686, an average of 448 patients per month. Over this period, 170 patients $(6.3 \%)$ were found to have atrial fibrillation (an average of 28 patients per month), with $100(59 \%)$ female and 70 (41\%) male patients (Figure 2.1). Their mean age was 73.5 years, with an age range of 38 - 95 years (s.d. 10.6). One hundred and two $(60 \%)$ patients were previously noted to have atrial fibrillation (including $10(3.5 \%)$ 
with a history of paroxysmal atrial fibrillation), whilst $68(40 \%)$ were 'newly diagnosed' patients, with no previous record of the arrhythmia.

Past medical history and underlying cause(s) of atrial fibrillation

Seventy-nine (46.5\%) of the 170 patients with atrial fibrillation had a history of ischaemic heart disease. Thirty (17.6\%) had a history of hypertension; $26(15.3 \%)$ rheumatic heart disease, predominantly mitral valve disease; and 6 (3.5\%) dysthyroid disease (Figure 2.2). In addition, 38 patients (22.4\%) of the 170 patients with atrial fibrillation had prior a history of cerebrovascular disease (stroke or transient ischaemic attack).

\section{Symptoms and presenting features on admission}

The commonest presenting symptoms were typical of cardiorespiratory symptomatology. Of the 170 patients, $88(51.8 \%)$ presented with dyspnoea, $58(34.1 \%)$ with chest pain, $44(25.9 \%)$ with palpitation, and $32(18.8 \%)$ with dizziness or syncope.

The predominant presenting clinical features included heart failure in 61 (35.9\%), a cerebrovascular event in 23 (13.5\%), myocardial infarction in $17(10 \%)$, angina pectoris in $14(8.2 \%)$, a chest infection in $12(7.1 \%)$ and digitoxicity in $3(1.8 \%)$ (Figure 2.3).

Investigations

All patients with atrial fibrillation had an electrocardiogram and chest $\mathrm{X}$-ray on admission. Recording of clinical data was variable, and complete information on investigations undertaken was only available for 146 patients (86\% of 170). Echocardiography was performed in 48 patients (33\%). Cardiac enzymes and thyroid function tests were performed in $92(63 \%)$ and $92(63 \%)$ respectively. A treadmill exercise test was performed in 5 patients (3.4\%) whilst 24 hour Holter monitoring was performed in only 8 patients $(5.5 \%)$

In the 170 patients with atrial fibrillation, a new myocardial infarction was identified on the electrocardiograph in 12 patients (7.1\%), whilst an old infarction was present in 15 patients $(8.8 \%)$. The commonest site was an inferior myocardial infarction, being 
present in 17 patients (63\% of the 27 patients with an infarct on the electrocardiograph). Cardiac ischaemic changes were found in 29 patients (17\% of all admissions).

Chest X-rays revealed cardiomegaly in 93 patients (54.7\%) of those admitted in atrial fibrillation and pulmonary congestion was present in 77 patients (45.3\%). Of the 48 patients in whom echocardiography was performed, findings included large left atria in $\mathbf{3 1}$ patients $(64.6 \%$ of 48$)$, mitral valve disease in $39(81.3 \%)$, poor left ventricular function in 30 (62.5\%) and left ventricular hypertrophy in $11(22.9 \%)$.

\section{Treatment prior to admission}

In those previously noted to be in atrial fibrillation (102 patients, including 10 with paroxysmal atrial fibrillation) the commonest therapy prior to admission was digoxin in 71 (70\%). A diuretic was administered in 89 (52.4\%), whilst a beta-blocker was used in 16 $(9.5 \%)$. Out of these 102 patients, antithrombotic therapy was used in only 37 patients, with $20(20 \%)$ patients being on warfarin therapy and $17(17 \%)$ on aspirin. Amongst the 17 patients on aspirin, it was prescribed to 11 patients for concomitant vascular disease rather than prophylaxis against thromboembolism.

\section{Treatment initiated following admission}

In the 68 patients ( $40 \%$ of 170 ) not previously noted to be in atrial fibrillation, digoxin was used in 51(75\%) whilst amiodarone was used on 23 (34\%) occasions. Additional therapy initiated in the group as a whole included a beta blocker in 6 patients (3.5\%) [for rate control in atrial fibrillation and treatment of associated angina] and diuretics, with intravenous diuretic required on admission in 52 patients (30.6\%), and subsequent maintenance oral diuretic required in 46 patients (27\%). Antithrombotic therapy was initiated in 27 patients. Seven patients (5\% of the 150 patients not previously on warfarin) were given warfarin, whilst 20 (15\% of the 138 patients not previously on aspirin) were commenced on aspirin therapy. Of these 20 patients only 5 were started on aspirin as prophylaxis against embolic events; the remainder (15 patients) were commenced on aspirin for concomitant vascular disease. Contraindications to anticoagulation were only identified in 49 patients $(34 \%$ of those not on 
warfarin); and these included peptic ulcer or gastrointestinal bleeding in $18(12 \%)$, dementia in $8(6 \%)$, chronic renal failure/dialysis in $8(6 \%)$, Crohn's Disease in $1(0.6 \%)$, alcohol excess in $4(3 \%)$ and other major illness in $10(7 \%)$.

Cardioversion was attempted in 21 cases (12.4\% of all admissions). Pharmacological cardioversion was the commonest method, being used in 19 patients $(11.2 \%)$, particularly in the coronary care setting. Of these, 18 patients were given amiodarone, whilst one patient cardioverted with intravenous digoxin. Two patients failed to cardiovert. The remaining 2 patients (1.2\%) underwent electrical (direct current) cardioversion.

Length of stay and inpatient mortality

Complete information on length of stay was available for 146 patients $(86 \%$ of 170). The median inpatient stay was 10 days, with a range of 1 - 154 days (IQR 6-19), largely due to patients with stroke. There was a weak but significant positive correlation between age and length of stay (Spearman $r=0.22 ; p=0.007$ ).

There were a total of $26(15.3 \%)$ inpatient deaths amongst these patients. The predominant causes of death were ischaemic heart disease and heart failure. No postmortem examinations were undertaken.

Discussion

The major reasons for treating atrial fibrillation are the prophylaxis of thromboembolic events and heart rate control, as a rapid ventricular rate may cause symptoms of angina, congestive heart failure or hypotension. Treatment of atrial fibrillation also improves overall cardiac function and exercise tolerance. However there is often wide variation in the management of this arrhythmia amongst physicians, both in the choice of investigations, and on specific aspects of treatment, such as the initiation of anticoagulant therapy (Chang et al 1990).

Standard investigations in a patient with atrial fibrillation should include an assessment of history, clinical state and possible precipitating factors (alcohol, infection, etc); 
thyroid status by thyroid function tests, and an assessment of cardiac status using the electrocardiograph (ECG), chest X-ray and echocardiogram. This latter investigation will also provide information on cardiac function and structural heart disease. In addition, measurement of cardiac enzymes (in an acute presentation) and 24hour Holter monitoring may also be required.

This study demonstrates a suboptimal application of these standard investigations in the management of patients with atrial fibrillation. For example, echocardiography was performed only in $33 \%$ of patients. Interestingly, of these patients, $65 \%$ had dilated left atria, $81 \%$ had mitral valve disease and $63 \%$ had poor left ventricular function; indicating that useful information was obtainable from this investigation. In addition, the high proportion of abnormal findings suggests a possible underutilization of the technique. Echocardiography is an important investigation, as demonstrated in analysis of echocardiographic data from the Stroke Prevention in Atrial Fibrillation study, where left ventricular dysfunction (on the 2-dimensional echocardiogram) and the size of the left atrium (from M-Mode echocardiograms) were the strongest independent predictors of later thromboembolism in patients with atrial fibrillation (Stroke Prevention in Atrial Fibrillation Investigators 1992b). In a further prospective echocardiographic study of 153 patients with thromboembolism, cardiac abnormalities were detected in 58\% (by transthoracic and transoesophageal echocardiography) and in these patients, the incidence of left-sided thrombi was increased in patients with atrial fibrillation when compared with those in sinus rhythm (Hofmann et al 1990). Echocardiography should therefore be a mandatory investigation in all patients with atrial fibrillation, with a significant contribution to thromboembolic risk stratification (Stroke Prevention in Atrial Fibrillation Investigators 1992b).

In addition, 24 hour Holter monitoring was performed in only $5.5 \%$ of patients although $18.8 \%$ had a history of dizziness or syncope. This has important implications as prolonged ventricular standstill may commonly occur in patients with controlled atrial fibrillation who complain of dizziness or syncope (Rebello and Brownlie 1987). A majority of these patients will benefit from permanent cardiac pacing (Rebello and Brownlie 1987). Another mandatory investigation, thyroid function tests, was performed in only $63 \%$ of our patients. 
This also has important implications as atrial fibrillation commonly complicates hyperthyroidism and clinically occult thyrotoxicosis may be the cause of 'idiopathic atrial fibrillation in many cases (Forfar and Toft 1979).

Although the commonest underlying pathology in our patients was ischaemic heart disease, ischaemic non-infarct changes were seen on the electrocardiogram in only $17 \%$ of patients, whilst infarction changes were present in a similar proportion (16\%). Atrial fibrillation was present in association with acute myocardial infarction in $7 \%$ of patients in this study. This proportion is slightly lower than previous observations (10-15\%), but the presence of the arrhythmia is pertinent in this situation as it is a marker of underlying ventricular dysfunction and a compromised myocardium (Pasternak et al 1993). This is in part related to left atrial ischaemia, the loss of atrial contribution to left ventricular filling, the increased ventricular rate with the arrhythmia and the association with anterior (rather than inferior) infarctions (Pasternak et al 1993). The presence of atrial fibrillation in acute myocardial infarction is therefore a marker of a worsened prognosis and increased mortality, particularly in patients with anterior wall infarction (Pasternak et al 1993). However, as the arrhythmia is more common in patients with extensive infarctions (and a poorer prognosis), there may be no independent effect of atrial fibrillation on mortality rate (Goldberg et al 1990, Pasternak et al 1993).

The frequency with which heart failure accompanies atrial fibrillation is shown in this study. For example, $52 \%$ of our patients presented with dyspnoea, pulmonary congestion was evident on the chest X-ray in $45 \%$ of patients, $63 \%$ of patients had poor left ventricular function on the echocardiogram and intravenous diuretic was required acutely on admission in $31 \%$ of patients (with subsequent maintenance oral diuretic being required in 27\%). Overall clinical heart failure was present in $35 \%$ of our patients. A similar proportion (37.5\%) of patients with atrial fibrillation has been demonstrated in a previous study of admissions with heart failure to a district general hospital (Parameshwar et al 1992) suggesting that my findings are quite typical. The presence of atrial fibrillation may also be a marker for an increased risk of death, particularly in heart failure patients (Middlekauff et al 1991). The presence of concomitant heart failure in patients with atrial fibrillation also appears to enhance 
the substantial risk for thromboembolism in these patients (Stroke Prevention in Atrial Fibrillation Study Group Investigators 1992).

Recent large scale, randomised, studies have established the importance of anticoagulation therapy in the prophylaxis against thromboembolic events in patients with chronic atrial fibrillation (Boston Area Anticoagulation Trial for Atrial Fibrillation Investigators 1990, Connolly et al 1991, Ezekowitz et al 1992, Petersen et al 1989, Stroke Prevention in Atrial Fibrillation Study Group Investigators 1990, Stroke Prevention in Atrial Fibrillation Investigators 1991). When my survey was performed only three of the five large scale studies had been published, but there was sufficient evidence that warfarin therapy reduced the risks of stroke by two-thirds in patients with chronic atrial fibrillation (Boston Area Anticoagulation Trial for Atrial Fibrillation Investigators 1990, Petersen et al 1989, Stroke Prevention in Atrial Fibrillation Study Group Investigators 1990). Despite this evidence, consensus about initiating oral anticoagulation therapy appears to be widely differing in view of the rate of anticoagulation in this study being surprisingly low, with only $5 \%$ of patients having warfarin added to their therapy. A suboptimal rate of prescribing warfarin was also shown in a retrospective audit of antithrombotic therapy use in 95 patients with atrial fibrillation (Bath et al 1993). The main concerns about initiating warfarin appear to be the inconvenience and safety (Lowe 1992). The quality of life for patients on anticoagulants was one aspect further studied in the BAATAF study and no significant differences between warfarin-treated and controls were found on measures of functional status, well-being, and health perceptions; unless complicated by a bleeding episode (Lancaster et al 1991). Only $6.5 \%$ of patients in this study claimed that warfarin restricted their lifestyle, although $13.1 \%$ admitted to worrying about their side-effects (Lancaster et al 1991). However, these findings may not be applicable to all patients on warfarin. For example, the average age in the BAATAF was 68 years, and younger, sports-active patients may find long-term warfarin therapy restrictive and an inconvenience. Further studies on this group of patients may be required.

Age should not be a contraindication to initiating oral anticoagulant therapy (Boston Area Anticoagulation Trial for Atrial Fibrillation Investigators 1990, Gurwitz et al 1988, Kutner et al 1991, Lancaster et al 1991). One survey of physicians demonstrated, however, 
that $51.4 \%$ believed that in elderly patients with atrial fibrillation, the risk of haemorrhage associated with warfarin outweighed the benefit; whilst $24.3 \%$ were not convinced of the effectiveness of warfarin in preventing of strokes in these patients (Kutner et al 1991). In fact, the Havard Aging Study showed that the risk of haemorrhage associated with warfarin was no greater in elderly than in younger patients (Gurwitz et al 1988). Nonvalvular atrial fibrillation is also an important cause of fatal massive cerebral infarction in the elderly, suggesting that primary prophylaxis, as well as secondary prevention, against stroke should always be considered for these patients (Yamanouchi et al 1989). The main reason for variation amongst physicians in initiating such therapy appears to be the perceived differences in the estimated risk of systemic embolism for individual patients (Chang et al 1990, Kutner et al 1991). The availability of a suitable marker of increased thrombogenesis may therefore assist physicians in the risk stratification and allow identification of high risk patients with atrial fibrillation whom would particularly benefit from anticoagulant therapy - this aspect is further investigated in this thesis.

Aspirin is thought to be an alternative to warfarin as thromboprophylaxis particularly in patients with previous stroke or myocardial infarction (Antiplatelet Trialists' Collaboration 1988). Aspirin was only initiated in 20 patients in this study, although the majority of use was for concomitant vascular disease rather than prophylaxis against thromboembolism. However, only two of the five trials of warfarin in atrial fibrillation have reported a reduction (by about $25 \%$ ) of thromboembolic risk with aspirin (Petersen et al 1989 , Stroke Prevention in Atrial Fibrillation Study Group Investigators 1990, Stroke Prevention in Atrial Fibrillation Investigators 1991). The use of aspirin as an effective antithrombotic agent for atrial fibrillation therefore remains controversial (Lowe 1992).

The haemodynamic disturbance of atrial fibrillation results essentially from the absence of atrial systole and from the rapidity and irregularity of the ventricular response. With increasing age and/or heart failure, atrial systole contributes an increasing amount towards overall stroke volume. Indeed there is usually a significantly improved cardiac output and exercise capacity following cardioversion of atrial fibrillation to sinus rhythm (Lipkin et al 1988). Following cardioversion, there is also the additional advantage of avoiding the long-term 
thromboembolic risk associated with atrial fibrillation. In our study, there was a low rate of attempted cardioversion (12\% of patients), with pharmacological cardioversion being the commonest method. Digoxin was also used in one patient for cardioversion despite evidence that it is no better than placebo (Falk et al 1987). For a patient in atrial fibrillation, the most complete relief of symptoms often occurs when sinus rhythm is restored. Cardioversion should therefore have been considered for most patients presenting with recent-onset atrial fibrillation. The procedure is generally most successful in younger patients with no underlying cardiac disorder and has the added advantage of being able to withdraw warfarin (for example, after two to four weeks (Dunn et al 1989)) if sinus rhythm is maintained.

This study establishes the importance of atrial fibrillation as a presenting arrhythmia amongst emergency medical admissions in the setting of a Scottish district general hospital. As complete information on length of stay and investigations undertaken was available in only $86 \%$ of patients, this study also highlights some of the problems which occur in producing accurate figures from admissions. This survey of atrial fibrillation provides useful information about a common condition and is particularly important at this time when there is an increasingly important role for medical audit in planning the allocation of resources. For example, the median inpatient stay in my study was 10 days, but with a wide range of between 1 to 154 days. This finding and the significant correlation between age of patient and length of inpatient stay has important implications particularly as the general population becomes more elderly (with a greater life expectancy) and in view of the increased prevalence of atrial fibrillation with age, for example, increasing from $0.5 \%$ at $50-59$ years to $8.8 \%$ at $80-89$ years (Wolf et al 1991).

In conclusion, atrial fibrillation is a common finding amongst emergency medical admissions to a district general hospital. Heart failure is the commonest associated clinical feature, whilst ischaemic heart disease is the most frequent underlying cause. There is also a suboptimal application of standard investigations for patients with atrial fibrillation, and a reluctance to commence oral anticoagulant therapy or to consider cardioversion, both of which are playing an increasingly prominent role in the management of this group of patients. 
These issues need to be addressed in order to optimise management of this common arrhythmia. 


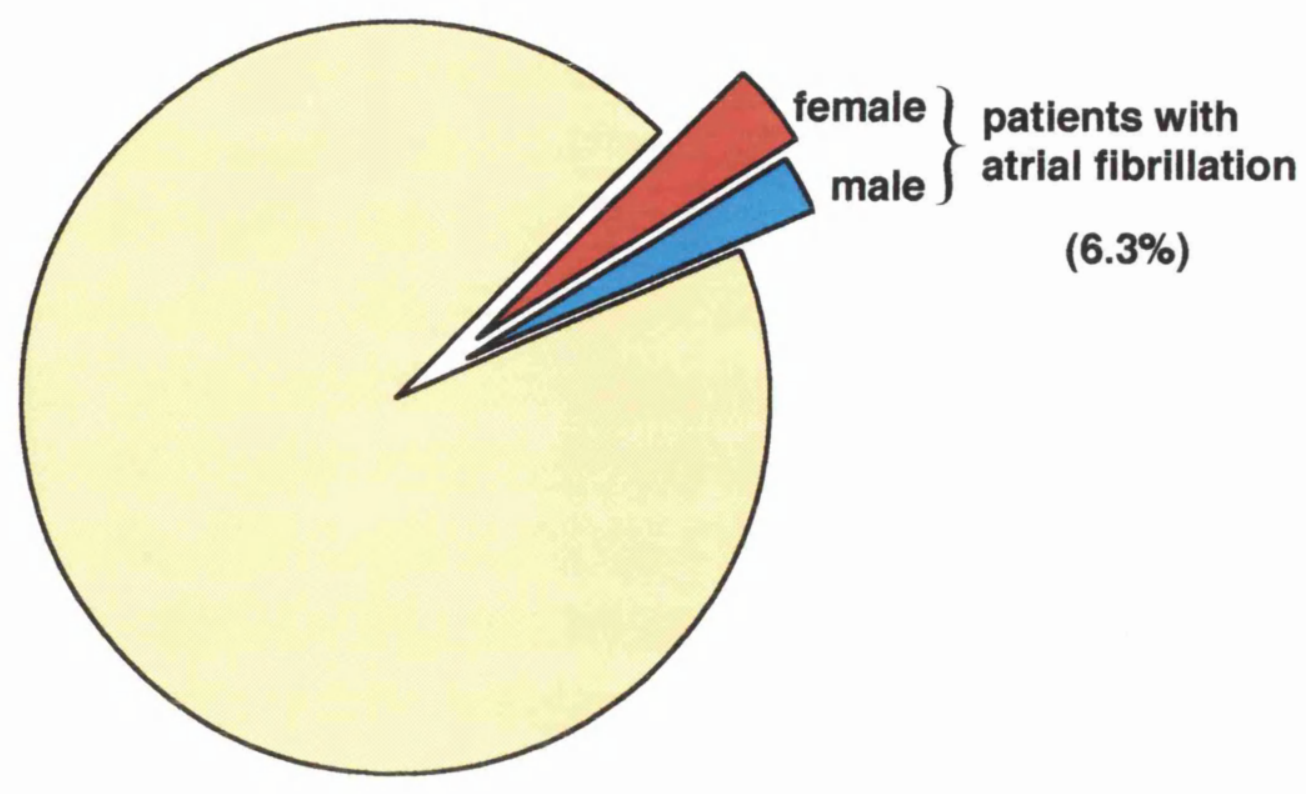

Total admissions

2686

FIGURE 2.1

EMERGENCY ADMISSIONS WITH ATRIAL FIBRILLATION TO A DISTRICT GENERAL HOSPITAL

(six months) 


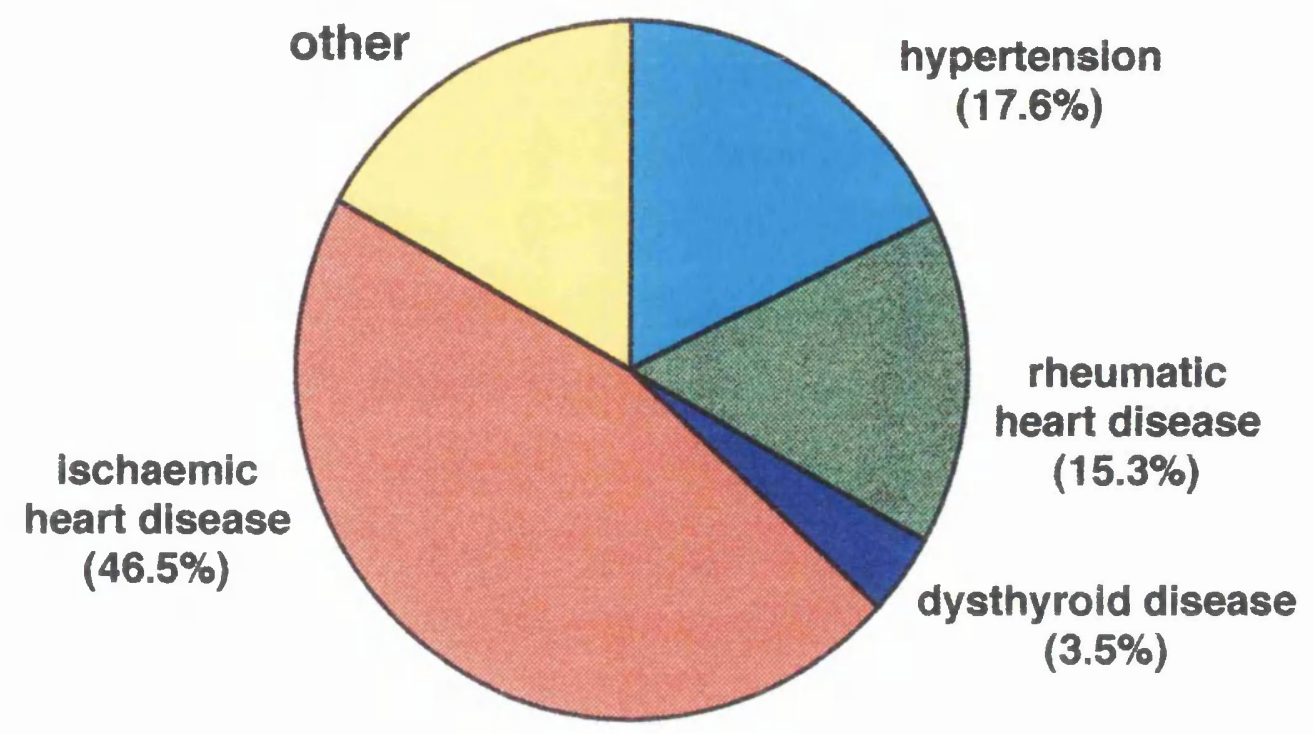

FIGURE 2.2

\section{UNDERLYING AETIOLOGY OF ATRIAL FIBRILLATION}

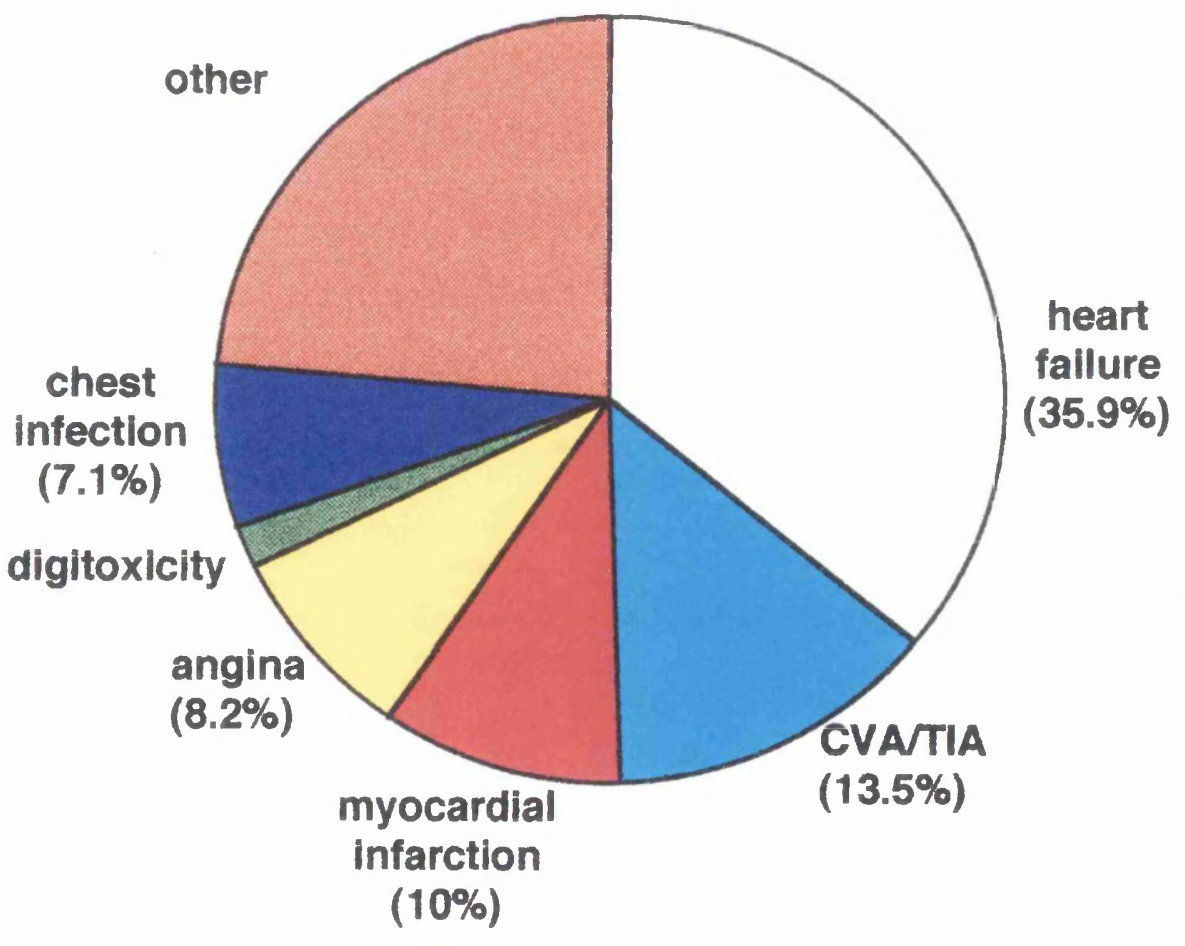

FIGURE 2.3

PRESENTING CLINICAL FEATURES ON ADMISSION 
CHAPTER 3

\section{THROMBOGENSIS IN CHRONIC ATRIAL FIBRILLATION}




\subsection{STUDIES OF PLASMA FIBRINOGEN, VON WILLEBRAND FACTOR AND FIBRIN D-DIMER IN CHRONIC ATRIAL FIBRILLATION}

Introduction

Chronic atrial fibrillation is associated with an increased incidence of stroke and other thromboembolic events. Within the broad spectrum of patients with atrial fibrillation, the risk for thromboembolism is not uniform, and subgroups at greater or less risk clearly exist. However, the mechanisms of thromboembolism in patients with atrial fibrillation have not been fully elucidated, and are likely to be multifactorial. The risk of thrombus formation and embolic events is, for example, higher with left atrial enlargement (Hinton et al 1977, Daniel et al 1988). However, atrial fibrillation per se can lead to and perpetuate atrial enlargement (Sanfillipo et al 1990). An enlarged left atrium may contribute to an increased thrombotic tendency as a result of abnormal flow patterns within the left atrium (Daniel et al 1988).

Abnormalities of plasma fibrinogen and von Willebrand factor are associated with thromboembolism. Elevated plasma fibrinogen levels are, for example, associated with an increased risk of cardiovascular disease, including ischaemic heart disease and stroke (Ernst 1991, Ernst and Resch 1993, Hamsten et al 1986, Meade et al 1980, Wilhelmsen et al 1984). They may promote disease by increasing fibrin formation, platelet aggregation or by rheological effects (blood viscosity, red cell aggregation) which may promote disturbed blood flow. Plasma WWF is a marker of endothelial dysfunction which is associated with thromboembolic stroke (Uchiyama et al 1983) and atherosclerosis (Mettinger 1982). Another plasma factor, the D-dimer fibrin fragment, reflects intravascular clotting levels by identifying the presence of cross-linked fibrin degradation products without interference from fibrinogen or non-cross-linked fibrin. This means that fibrin D-dimer is a marker of intravascular thrombus formation and not just a reflection of plasma fibrinogen levels. Elevated levels of fibrin degradation products are found in conditions associated with intravascular (and sometimes extravascular) activation of the coagulation system, including those with a predisposition to venous thrombosis (Hunt et al 1985). In addition, two recent studies have suggested that plasma levels of D-dimer are increased in patients with atrial fibrillation, whether or not co-existing structural heart disease is present, when compared to 
patients in sinus rhythm (Gustafsson et al 1990, Kumagai et al 1990). Abnormalities in these plasma factors may in part account for the variable risk of thromboembolism in different subgroups of patients with atrial fibrillation.

Five major, prospective studies have demonstrated that warfarin reduces the incidence of thromboembolic complications in patients in atrial fibrillation (Boston Area Anticoagulation Trial for Atrial Fibrillation Investigators 1990, Connolly et al 1991, Ezekowitz et al 1992, Petersen et al 1989, Stroke Prevention in Atrial Fibrillation Investigators 1991). The effect of aspirin however is less certain (Lowe 1992).

This study was performed to determine if chronic atrial fibrillation is associated with abnormalities in plasma fibrinogen, vWF or D-dimer levels; and if so, whether or not such levels were related to the haemodynamic disturbance within an enlarged left atrium, with poor left ventricular function, or to existing treatment with warfarin or aspirin.

\section{Materials and methods}

Patients- $\quad$ The design was a prospective population controlled study of male and female patients with chronic atrial fibrillation. Chronic atrial fibrillation was defined as atrial fibrillation documented electrocardiographically on two occasions at least 48 hours apart during the same inpatient stay, or on at least two visits to the outpatient clinic. Excluded were those patients aged over 75 and those with significant systemic illness (which influences fibrinogen, vWF and fibrin Ddimer levels) such as renal failure, liver impairment (defined as abnormal liver function tests with aspartate transaminase (AST) or alanine transaminase (ALT) levels more than twice the upper limit of normal), chronic infections or neoplastic disease. Patients with hypertension, limited venous access, those requiring transfusion or those actively bleeding were also excluded.

Results were compared to those of 158 population controls aged 55-64 years in sinus rhythm, sampled from a local population survey (Second North Glasgow MONICA Study; Lowe et al 1992; Lowe et al, in preparation (for D-dimer and vWF levels)). For patients taking warfarin therapy, a Thrombotest was performed to ensure satisfactory anticoagulation and to establish the intensity of effect of warfarin. 
Echocardiography- Two dimensional and Doppler echocardiography were performed with the patient in the left lateral decubitus position using a Hewlett-Packard Sonos 500 machine (Hewlett-Packard, Arondale, Pa, USA) equipped with a standard $2.5 \mathrm{MHz}$ transducer. Images were obtained in the standard parasternal long axis, parasternal short axis, and apical fourchamber planes. The transducer was angled to maximise cardiac chamber size and gain positions were adjusted to obtain the clearest outline of the endocardium. Doppler examination was performed using colour-flow, pulsed and continuous wave modes. Using the standard video facility of the ultrasound machine, off line analytical methodology was used to quantify measurements. In the parasternal long-axis view, the anteroposterior dimension of the left atrium was measured and in the standard apical four-chamber view, the superoinferior (base-to-roof) and mediolateral (transverse) dimensions of the left atrium were measured (Sanfillipo et al 1990) (Figures 3.1, 3.2). Left atrial volume was calculated using a standard ellipsoid formula (Figure 3.3) (Sanfillipo et al 1990). The apical four chamber view was used to measure the right atrium superoinferior and mediolateral dimensions and the volume estimated using the same ellipsoid formula, assuming anteroposterior dimension being equal to the mediolateral dimension (Figures 3.1, 3.2) (Sanfillipo et al 1990). All measurements were taken at maximum separation of chamber walls at end-systole (that is, at the video frame just preceding mitral valve opening), using the leading edge to leading edge convention. Left ventricular dimensions were measured using $\mathrm{M}$ mode echocardiography in the parasternal long axis view using the technique recommended by the American Society of Echocardiography (Sahn et al 1978) and the fractional shortening calculated as an index of left ventricular function (Figure 3.4).

All echocardiography was performed by one operator blinded to the patients clinical details. Echocardiography data were compared to 14 healthy volunteers who served as 'normal controls. To allow validation of the measurements of atrial and ventricular dimensions, and to assess reproducibility, interobserver and intraobserver variability, a sample of 20 patients selected at random were studied twice on different occasions by the same observer (myself) and another 20 subjects on different occasions by two different observers (myself and one other observer). 
Blood samples-

Blood samples were drawn from the antecubital vein by careful venipuncture, anticoagulated with trisodium citrate $(0.11 \mathrm{M}, 9: 1 \mathrm{v}: \mathrm{v})$ and centrifuged. The plateletfree plasma was immediately separated, frozen to $-40^{\circ} \mathrm{C}$, and assayed in batches within 4 weeks of collection.

Assay procedures- Plasma D-dimer antigen levels were measured using an ELISA method (AGEN, Parsippany, NJ, USA). Plasma fibrinogen was assayed by the Clauss assay, using a CoagA-mate coagulometer and Organon Teknika reagents and standards (Organon Teknika, Cambridge, UK). Plasma von Willebrand factor was assayed using an ELISA method (ASSERACHROM, Diagnostica Stago, France). The sensitivity of the assays used allowed measurement of plasma D-dimer, vWF and fibrinogen levels to a minimum of $30 \mathrm{ng} / \mathrm{ml}, 1 \%$ and 0.5 g/l respectively. The reproducibility of all three methods allowed a coefficient of variation of less than $5 \%$.

Statistical analysis- All the values of the rheological measurements and cardiac dimensions were expressed as median \pm interquartile range (IQR). Statistical comparison was performed using ANOVA and t-tests for normal distributions and the Kruskal-Wallis test and Mann-Whitney two sample rank test for non-parametric distribution, and $95 \%$ confidence intervals (c.i.) were calculated for the point estimate of the difference in medians. Correlations between clinical variables or rheological measurements were performed using Spearman's Rank correlation method. Stepwise multiple regression analyses were performed with plasma fibrinogen and fibrin D-dimer as the dependent variables to determine whether age, smoking habit, left atrial volume (ellipsoid), right atrial volume, left ventricular fractional shortening and body mass index contributed significantly to levels of these coagulation parameters. A probability of $\leq 0.05$ was considered statistically significant.

Reproducibility for echocardiographic measurements was expressed as mean difference $\pm 2 s . d$ (Bland and Altman 1986). A one-sample t-test test was performed to determine whether the mean difference was significantly different from zero. All statistical calculations were 
performed on a microcomputer using a commercially available statistical package (MINITAB v8, Minitab INC, PA,USA).

\section{Results}

Patient groups- $\quad$ A total of 85 patients (42 male and 43 female; mean age 63.1 years, s.e.m. 1.01) with chronic atrial fibrillation were studied. At the time of the study, 36 were taking no antithrombotic therapy (Group 1), 31 were taking warfarin (including 2 on both warfarin and aspirin therapy) (Group 2) and 18 were taking aspirin alone (Group 3). They were compared to 158 population controls derived from a pre-existing random healthy population sample, the 2nd MONICA study. Clinical variables in these groups are as shown in Table 3.1. Twenty patients (23.5\%) were still smoking. There were no differences in sex ratio $\left(X^{2}=4.79,3\right.$ d.f.; $\left.p=0.19\right)$ and proportions of smokers ( $X^{2}=7.36,3$ d.f.; $\left.p=0.06\right)$ between the groups studied.

Underlying aetiology - Of the 85 patients with atrial fibrillation, 73 were found to have a predisposing factor to atrial fibrillation. Valvular heart disease was the commonest factor $(n=34)$, followed by ischaemic heart disease ( $n=30$, including 10 patients with a clinical history of both valuular heart disease and ischaemic heart disease). Other causes included patients with dilated cardiomyopathy ( $n=3)$, predominantly idiopathic or alcoholic, but 12 patients did not have any obvious predisposing factors. There were 31 patients taking warfarin and all Thrombotest results were within the therapeutic range (5-15\%).

Plasma fibrinogen, von Willebrand factor and fibrin D-dimer levels Fibrinogen levels were measured in all 85 patients (median $3.58 \mathrm{~g} / \mathrm{l}, \mathrm{IQR} 3.0-4.7$ ) but due to technical problems, plasma D-dimer and von Willebrand factor levels were only measured in 81 patients (D-dimer: median 89 ng/ml, IQR 50-193) and 67 patients (vWF: median $158 \%$, IQR 114-200) respectively. As a group, the patients with atrial fibrillation had significantly higher levels of fibrinogen than the population controls (Kruskal-Wallis test $H=78$, d.f. $=3, p<0.0001$; Mann-Whitney test, point estimate of difference between medians $1.23 \mathrm{~g} /$; $95 \%$ confidence interval (c.i.) 1.01 to $1.55, p<0.0001$ ) and of vWF (Kruskal-Wallis test $H=27$, d.f. $=3, p<0.0001$; Mann-Whitney test, point estimate of 
difference between medians $63 \%$; 95\% confidence interval (c.i.) 47 to $79, p<0.0001$ ) when compared to population controls. The correlations between plasma fibrinogen and D-dimer (Spearman $r=0.23, p=0.03)$ and between vWF and D-dimer $(r=0.29, p=0.01)$ were however weak.

In Group 1 (no antithrombotic therapy) ( $n=36)$, plasma fibrinogen remained significantly elevated compared with population controls (point estimate of difference between medians $1.23 \mathrm{~g} / \mathrm{l} ; 95 \%$ c.i. 0.88 to $1.62, \mathrm{p}<0.0001$ ) (Table 3.2, Figure 3.5). There was also a significant elevation of plasma D-dimer levels (point estimate of difference between medians 77 $\mathrm{ng} / \mathrm{ml} ; 95 \%$ c.i. 38 to $122, p<0.01$ ) and vWF (point estimate of difference between medians $63 \%$; $95 \%$ c.i. 38 to $89, p<0.0001$ ) in these patients when compared to population controls (Table 3.2; Figure 3.6, 3.7). There were no significant correlations between these coagulation parameters and atrial size or ventricular function (Table 3.4).

In Group 2 (warfarin therapy), there was no significant difference in plasma fibrinogen (point estimate of difference between medians $0.14 \mathrm{~g} / \mathrm{l} ; 95 \%$ c.i. -0.44 to $0.77, p=0.65$ ) or VWF (point estimate of difference between medians $3.5 \%$; $95 \%$ c.i. -41 to $41, p=N S$ ) when compared to patients in Group 1 (Table 3.2; Figure 3.5, 3.7). However, there was a significant difference in plasma D-dimer levels between the two groups (point estimate of difference between medians $90 \mathrm{ng} / \mathrm{ml}, 95 \%$ c.i. 39 to 150, p<0.0001) (Table 3.2, Figure 3.6). There were no significant correlations between the coagulation parameters and left atrial volume or ventricular function (Table 3.4).

In Group 3 (aspirin therapy alone) $(n=18)$, there were no significant differences in plasma fibrinogen (point estimate of difference between medians $0.08 \mathrm{~g} / \mathrm{l} ; 95 \%$ c.i. -0.52 to 0.77 , $p=0.73$ ), D-dimer (point estimate of difference between medians $-34 \mathrm{ng} / \mathrm{ml} ; 95 \%$ c.i. -114 to 21.0 , $p=0.25$ ) or vWF (point estimate of difference between medians $2 \% ; 95 \%$ c.i. -35 to $41, p=N S$ ) levels when compared to Group 1 (Table 3.2; Figure 3.5,3.6, 3.7). In Group 1 and Group 3 patients, there was a significant positive correlation between plasma fibrin D-dimer and VWF levels $(p=0.52 . p<0.001)$. 
Influence of underlying aetiology, left atrial size and left ventricular function -

As plasma fibrinogen, VWF and fibrin D-dimer levels are similar in patients with and without aspirin therapy, but not on warfarin (that is, Group 1 and Group 3), further subgroup analysis was performed on the 68 patients in these two groups with regard to the influence of underlying aetiology, atrial size and left ventricular function.

There were no significant differences for plasma fibrinogen (Kruskal-Wallis test $H=3.06, p=0.38$ ), fibrin D-dimer levels (Kruskal-Wallis test $H=3.27, p=0.35$ ) or WWF (Kruskal-Wallis test $\mathrm{H}=11.9, \mathrm{p}=0.064$ ) between patients with and without underlying heart disease. In particular, there were no statistically significant differences in plasma fibrinogen levels when patients with valve disease were compared to those with no obvious aetiology (Mann-Whitney test, point estimate of difference between medians $-0.52 ; 95 \%$ c.i. -1.2 to $0.56, p=0.34$ ) or when patients with ischaemic heart disease were compared to those with no obvious aetiology (point estimate of difference between medians $-0.99 \mathrm{~g} /$; $95 \%$ c.i. -1.64 to $0.11, p=0.09$ ). Similarly, there were no differences in plasma D-dimer levels when those with valve disease were compared to those with no obvious aetiology (point estimate of difference between medians $-27 \mathrm{ng} / \mathrm{ml}$; $95 \%$ c.i. -179 to $66, p=0.81$ ) or when patients with ischaemic heart disease were compared to those with no obvious aetiology (point estimate of difference between medians $-5.5 \mathrm{ng} / \mathrm{ml} ; 95 \%$ c.i. -132 to 77 , $\mathrm{p}=0.83$ ). There were also no differences in plasma vWF levels when those with valve disease were compared to those with no obvious aetiology (Mann-Whitney test, point estimate of difference between medians $-38 \%$; $95 \%$ c.i. -95 to $42, p=0.53$ ) or when patients with ischaemic heart disease were compared to those with no obvious aetiology (point estimate of difference between medians $-16 \%$; $95 \%$ c.i. -99 to $65, p=0.40$ ).

Technically adequate left and right atrial measurements were obtained in 81 and 77 patients respectively. However, due to technical difficulties, left ventricular dimensions were only obtained in 72 patients. Echocardiographic measurements and dimensions are summarised in Table 3.3. Echocardiographic data $(n=54)$ for patients not on warfarin (Group 1 and 3) were related to plasma fibrinogen, WWF and D-dimer. There were no significant correlations between left atrial dimension, left atrial volumes (ellipsoid) and left ventricular fractional shortening, and plasma fibrinogen, VWF or fibrin D-dimer levels. There was a weak negative correlation between 
plasma fibrinogen and right atrial volume (Spearman $r=-0.3, p=0.03$ ). In addition, amongst these patients, there were no differences in plasma fibrinogen (point estimate of difference between medians $0.18 \mathrm{~g} / \mathrm{l} ; 95 \%$ c.i. -0.38 to $0.95, \mathrm{p}=0.48$ ) or fibrin D-dimer levels (point estimate of difference between medians $-25 \mathrm{ng} / \mathrm{ml} ; 95 \%$ c.i. -76 to $47, \mathrm{p}=0.29$ ) when patients with a left atrial dimension $>4.2 \mathrm{~cm}$ were compared to those with a left atrial dimension of $<4.2 \mathrm{~cm}$. There was, however, a higher plasma vWF in patients with a left atrium of $>4.2 \mathrm{~cm}$ when compared to those with a left atrium $<4.2 \mathrm{~cm}$ (point estimate of difference between medians $42 \%$; $95 \%$ c.i. 3 to 87 , $\mathrm{p}=0.045$ ). There were no differences in plasma fibrinogen (point estimate of difference between medians $0.29 \mathrm{~g} /$; $95 \%$ c.i. -0.25 to $1.06, p=0.34$ ), fibrin D-dimer levels (point estimate of difference between medians $23 \mathrm{ng} / \mathrm{ml}$; $95 \%$ c.i. -29 to $112, \mathrm{p}=0.47$ ) or vWF (point estimate of difference between medians $14 \%$; $95 \%$ c.i. -32 to $63, p=0.49$ ) when patients with a left ventricular fractional shortening of $<25 \%$ were compared to those with a normal fractional shortening (FS >25\%).

For patients not on warfarin, stepwise multiple regression analysis with plasma fibrin D-dimer as the dependent variable demonstrated that when age, left atrial volume (ellipsoid), right atrial volume, left ventricular fractional shortening, smoking habit, body mass index and plasma fibrinogen were considered, only body mass index $(t=3.65, p<0.05)$ was a significant predictor for plasma fibrin D-dimer. Similar stepwise multiple regression analysis with plasma fibrinogen and VWF as dependent variables revealed no significant predictors among age, left atrial volume (ellipsoid), right atrial volume, left ventricular fractional shortening, smoking habit and body mass index. Amongst these patients there was a weak correlation between age and vWF (Spearman $r=0.31, p=0.02)$ and plasma fibrinogen and body mass index $(r=-0.36, p=0.001)$.

Reproducibility of 2-dimensional and M-mode echocardiographic measurements These are summarised in Table 3.5. There was overall good agreement between inter- and intraobserver measurements. 
Discussion

Chronic atrial fibrillation has been established to carry an annual risk of 3-6\% of thromboembolic complications, this being 5-7 times greater than that of controls in sinus rhythm (Peterson 1990). The female sex, smoking, heart failure, enlarged left atrial size, poor left ventricular function and a history of previous embolic episodes are additive risk factors. This was demonstrated in the Stroke Prevention in Atrial Fibrillation study where the presence of recent congestive heart failure, hypertension and previous thromboembolism were independent clinical risk factors for subsequent thromboembolism (Stroke Prevention in Atrial Fibrillation Investigators 1992a). The presence of these three independent clinical predictors defined patients with rates of thromboembolism of $2.5 \%$ per year (no risk factors), $7.2 \%$ per year (one risk factor) and $17.6 \%$ (two or 3 risk factors) (Stroke Prevention in Atrial Fibrillation Investigators 1992a). In addition to an increase in stroke and thromboembolism, the size of the left atrium has also been considered to contribute to an increased risk of atrial thrombi and spontaneous echo contrast on transoesophageal echocardiography (Caplan et al 1986, Daniel et al 1988, Manning 1993, SPAF Investigators 1992b). Echocardiographic data from the Stroke Prevention in Atrial Fibrillation study demonstrated that left ventricular dysfunction (on the 2-dimensional echocardiogram) and the size of the left atrium (from M-Mode echocardiograms) were strong independent predictors of later thromboembolism in patients with atrial fibrillation (Stroke Prevention in Atrial Fibrillation Investigators 1992b). These findings therefore point not only to the left atrium but also toward the left ventricle as a cause or consequence of the pathology responsible for thromboembolic risk in patients with atrial fibrillation. In addition, recent studies have also suggested that left atrial enlargement may be a consequence of the duration of atrial fibrillation (Sanfillipo et al 1990 , Petersen et al 1987, Sosa-Suarez et al 1989), although previous studies have suggested the converse, left atrial enlargement being a cause rather than a consequence of atrial fibrillation (Takahashi et al 1982).

Left atrial enlargement may also be associated with increased spontaneous echo contrast on transoesophageal echocardiography, suggesting flow disturbance (stasis or slow blood flow) and resulting in red cell aggregation within the enlarged atrial cavity (Black et al 1991, Chen et al 1990, Daniel et al 1988). One study in patients with mitral valve disease suggested that 
there was no single factor responsible for the formation of spontaneous echo contrast; and mitral stenosis, atrial fibrillation and dilated left atria were all necessary (Chen et al 1990). In contrast, one study of patients with nonrheumatic atrial fibrillation did not demonstrate any association between left atrial diameter (or left ventricular size/function) and the presence of the phenomenon (Tsai et al 1992). Spontaneous echo contrast itself appears to be predictive for left atrial thrombus and suspected embolism in patients with atrial fibrillation, and is unrelated to age, gender, left ventricular dysfunction or anticoagulant therapy (Black et al 1991, Castello et al 1990, Manning 1993, Tsai et al 1992). The rheological mechanism for spontaneous echo contrast is thought to involve the effect of fibrinogen, or its products, which under low flow and low shear rate conditions promote red cell aggregation and the rouleau formation of erythrocytes (Sigel et al 1981, Merino et al 1992). Spontaneous echo contrast therefore provides 'visual' evidence for a rheological mechanism for thrombogenesis in atrial fibrillation. Unfortunately, in this study, I was unable to assess the correlation of spontaneous echo contrast to rheological markers because the phenomenon was only demonstrated in one patient (on warfarin) using transthoracic echocardiography. This is not unexpected as transoesophageal echocardiography is more sensitive than transthoracic echocardiography in detecting spontaneous echo contrast (Chen et al 1990).

This study demonstrates that plasma levels of fibrinogen, fibrin degradation products (fibrin D-dimer) and von Willebrand factor (VWF) are increased in patients with chronic atrial fibrillation who are not taking any antithrombotic therapy, when compared to population controls of similar age in sinus rhythm. These results were not attributable to an excess of smokers in the atrial fibrillation group (Table 3.1); nor are they attributable to underlying cardiac disease, with no statistically significant differences between patients with an underlying cause for atrial fibrillation (ischaemic heart disease, valve disorders) when compared to those with no obvious cause. In this study, there was however a trend towards higher median plasma fibrinogen levels in patients with ischaemic heart disease. My findings in this study also suggest that it is atrial fibrillation itself which is the major contributory factor to the increased thrombotic tendency as reflected by the elevated fibrinogen and D-dimer levels, there being no significant correlation with atrial size or ventricular function. Similar findings were reported by two previous small studies of 
patients with atrial fibrillation (Gustafsson et al 1990, Kumagai et al 1990). The increased plasma fibrinogen levels in atrial fibrillation may promote thromboembolic events in patients with atrial fibrillation, either by increasing the tendency to thrombosis (as suggested by the correlation with plasma D-dimer levels)or by increasing flow disturbance through its rheological effects in increasing red cell aggregation and blood viscosity in areas of stasis, for example, in the dilated atria in patients with atrial fibrillation. In addition, there may be an interaction with underlying endothelial dysfunction, as reflected by the increased vWF levels in these patients and the positive correlation between VWF and fibrin D-dimer levels.

Although no significant correlations of plasma fibrinogen or D-dimer levels were found in association with left ventricular fractional shortening, this measurement poses certain problems. Firstly, there were insufficient numbers to fully assess a broad spectrum of left ventricular function. For example, only 5 patients in our study had a fractional shortening of $<10 \%$ (normally regarded as 'poor' left ventricular contractility). Additionally, the median fractional shortening in the whole study population was $21.5 \%$ (IQR 16.2 to $28.9 \%$ ) is just below lower limit of the normal range (>25\%) for our laboratory. Secondly, the measurement of fractional shortening is only a crude indicator of overall left ventricular function, as for example patients with a discrete apical wall motion abnormality may falsely appear to have normal ventricular function. There was, however, a weak negative correlation between plasma fibrinogen and left ventricular end-diastolic dimension, suggesting that some relationship may well exist with ventricular impairment. I therefore have reservations therefore about the lack of correlation between coagulation parameters and left ventricular function (as assessed by fractional shortening) in this study and this aspect will be the subject of a separate investigation.

Smoking is another factor associated with an increase in plasma fibrinogen, vWF and D-dimer levels (Petersen 1987, Ernst 1990). However, the proportion of smokers in population controls was $40 \%$ compared to $32 \%$ of patients with atrial fibrillation without any antithrombotic therapy (Group 1). Smoking should not be expected therefore to account for the elevation in plasma fibrinogen, WWF and D-dimer seen in Group 1. Patients on warfarin therapy (Group 2) and aspirin therapy (Group 3) had similar proportions of smokers (23\% and $16 \%$ respectively), which were lower than for Group 1. The difference between the proportions of 
smokers in different groups was however not statistically significant, but some contribution of smoking to the elevated D-dimer levels in Group 1 compared to Group 2 is possible.

My study demonstrates that patients with atrial fibrillation continue to have elevated plasma fibrinogen and vWF levels despite treatment with warfarin or aspirin. As other studies have demonstrated that elevated fibrinogen (Hamsten et al 1986) or WWF (Jansson et al 1991) levels are predictors of cardiovascular disease, these patients may continue to be at increased risk despite being adequately anticoagulated. Chronic atrial fibrillation itself may increase plasma fibrinogen levels, and there may be a contribution from the increased left atrial size. A number of patients in this study had underlying ischaemic heart disease $(n=30)$ and although this has been linked to elevated fibrinogen and VWF levels (Hamsten et al 1986, Wilhelmsen et al 1984), I do not feel that this was a significant contributory factor to the high levels of plasma fibrinogen and VWF in these patients. The main reasons for this include the demonstration that there is no significant difference in plasma fibrinogen and vWF levels in patients with atrial fibrillation with and without ischaemic heart disease. Secondly, observations in a large local population survey of patients (the 2nd North Glasgow MONICA Study) demonstrate that those with ischaemic heart disease and sinus rhythm had relatively minor elevations in plasma fibrinogen, vWF and D-dimer levels (Lowe et al 1992; Lowe et al, submitted for publication).

An important question is why is atrial fibrillation associated with an increased plasma fibrinogen or WWF level. It is unlikely that the increased fibrinogen or vWF levels are a secondary response to increased clot or thrombus formation, as in such cases D-dimer levels would have been elevated in the subgroups with and without warfarin therapy. Additionally, warfarin therapy is not known to influence any underlying pathological process and therefore fibrinogen or VWF levels would not have been expected to be different between the groups with and without warfarin therapy, as demonstrated here. Abnormalities in cardiac blood flow (for example, sluggish, slow flow within the atria) may be partly responsible, resulting in flow abnormalities and endothelial dysfunction (causing raised vWF) in the pulmonary vasculature. This latter effect may stimulate lung macrophages to produce hepatocyte stimulating factor, increasing the liver synthesis of fibrinogen (in a similar manner to smoking (Ritchie et al 1982). Further studies investigating the mechanisms by which fibrinogen and vWF synthesis are 
increased are needed. The precise mechanism for the elevated plasma fibrinogen and vWF levels is therefore uncertain.

As plasma D-dimer is a marker of intravascular cross-linked fibrin formation, my finding that plasma levels of D-dimer were significantly elevated in patients with atrial fibrillation (without any antithrombotic therapy), when compared to controls in sinus rhythm, suggests that there is ongoing intravascular fibrin formation in these patients. This finding is consistent with two previous small studies (Gustafsson et al 1990, Kumagai et al 1990). Additionally, in a small study of patients with atrial fibrillation and mitral stenosis, increased levels of other markers of a thrombotic state (fibrinopeptide A, thrombin-antithrombin III complex) have been demonstrated within the left atria of such patients (Yamamoto et al 1993). These thrombotic marker levels also did not correlate with the left atrial size, as in my study. If warfarin or aspirin therapy effectively prevented intravascular fibrin and thrombus formation, then D-dimer levels would be expected to be lower in patients on warfarin or aspirin therapy. This is indeed clearly demonstrated for warfarin in this study (which found that the median plasma D-dimer level was also reduced by about twothirds, consistent with the finding the warfarin therapy reduced thromboembolic risk by about twothirds, based on randomised clinical trials (range $35 \%$ to $86 \%$ )) (Lowe 1992, Stroke Prevention in Atrial Fibrillation Investigators 1992a). Patients on aspirin therapy had a lesser (approximately one-third) reduction in plasma D-dimer levels when compared to patients on no antithrombotic therapy but the difference was not statistically significant. This may be a reflection of either a lower antithrombotic efficacy of aspirin; or else the smaller number of patients within this group. In addition, the mechanisms of action of aspirin and warfarin differ. The former acts by inhibition of platelet function whilst the latter acts by the reduction of clotting factor synthesis. Larger studies of patients on aspirin are therefore required to assess risk reduction of thromboembolism in patients with chronic atrial fibrillation (current estimate is a risk reduction of about $25 \%$ ) (Lowe 1992).

Von Willebrand factor levels were also significantly increased in patients with chronic atrial fibrillation, irrespective of anticoagulant therapy. This finding is consistent with one previous small study of patients with atrial fibrillation (Gustafsson et al 1990). High vWF levels have previously also been associated with atherosclerosis and in particular, the recurrence of 
thromboembolic (rather than embolic) stroke (Mettinger et al 1982, Uchiyama et al 1983). My findings therefore suggest a role for VWF in the pathogenesis of thromboembolic risk in patients with chronic atrial fibrillation. As VWF is a marker of endothelial (which may extend to endocardial) dysfunction, this is consistent with Virchow's observation of the importance of the vessel wall in thrombogenesis (Virchow's triad) (Virchow 1856). The findings of a modest correlation between plasma vWF and fibrin D-dimer levels in patients not on warfarin therapy $(r=0.52, p<0.001)$, and higher VWF levels in patients with a large left atrium $(>4.2 \mathrm{~cm})$ are in support of this hypothesis.

Anticoagulant therapy carries a risk of bleeding, and to be able to select out those patients who would particularly benefit from warfarin (or aspirin) would be a major advance. My study suggests that measurement of plasma D-dimer may identify a subgroup of patients with atrial fibrillation (those with increased plasma D-dimer) who would particularly benefit from anticoagulant therapy. A prospective study of plasma D-dimer levels in a large cohort of patients with atrial fibrillation is required to test this hypothesis. Such a study might also assess the predictive value of plasma fibrinogen and vWF levels for thromboembolic complications. The apparent lack of effect of warfarin or aspirin therapy on plasma fibrinogen or WWF levels suggests that they could be used as risk markers even in patients taking antithrombotic therapy. For the present, however, this study confirms still further that anticoagulation should be considered for all patients with chronic atrial fibrillation as they appear to have increased intravascular thrombus generation. Prospective studies on the effects of introducing warfarin in these patients are required, as investigated in chapter 10 of this thesis.

In conclusion, my results confirm significantly elevated median plasma fibrinogen and VWF levels for patients with chronic atrial fibrillation. Plasma D-dimer levels were also elevated in patients with chronic atrial fibrillation whom were not on antithrombotic therapy, suggesting increased intravascular thrombogenesis in such patients. For patients taking warfarin therapy there was a strikingly lower level of circulating fibrin D-dimer, suggesting that warfarin therapy was effective in preventing intravascular thrombus formation. These results provide, therefore, a possible mechanism to both assess those patients with atrial fibrillation who are at high risk of thrombogenesis and also to measure the efficacy of anticoagulant therapy in reducing fibrin or thrombus formation. 
TABLE 3.1

\section{PATIENT CHARACTERISTICS}

\begin{tabular}{|c|c|c|c|c|}
\hline & $\begin{array}{l}\text { PATIENTS } \\
\text { GROUP } 1 \\
\text { no } \\
\text { anti-thrombotic } \\
\text { therapy }\end{array}$ & $\begin{array}{c}\text { WITH } \\
\text { GROUP } 2 \\
\text { warfarin }\end{array}$ & $\begin{array}{c}\text { CHRONIC AF } \\
\text { GROUP } 3 \\
\text { aspirin }\end{array}$ & $\begin{array}{c}\text { CONTROL } \\
\text { GROUP } \\
\text { (sinus } \\
\text { rhythm) }\end{array}$ \\
\hline$\underset{(n)}{\text { number }}$ & 37 & 31 & 19 & 158 \\
\hline$\underset{(\%)}{\text { males }}$ & $\begin{array}{c}19 \\
(51 \%)\end{array}$ & $\begin{array}{c}12 \\
(39 \%)\end{array}$ & $\begin{array}{c}13 \\
(68 \%)\end{array}$ & $\begin{array}{c}88 \\
(55 \%)\end{array}$ \\
\hline $\begin{array}{c}\text { smokers } \\
(\%)\end{array}$ & $\begin{array}{c}12 \\
(32 \%)\end{array}$ & $\begin{array}{c}7 \\
(23 \%)\end{array}$ & $\begin{array}{c}3 \\
(16 \%)\end{array}$ & $\begin{array}{c}64 \\
(40 \%)\end{array}$ \\
\hline $\begin{array}{c}\text { AGE (yrs) } \\
\text { mean (s.e.m.) }\end{array}$ & $\begin{array}{l}62.6 \\
(1.6)\end{array}$ & $\begin{array}{l}60.3 \\
(1.6)\end{array}$ & $\begin{array}{l}68.4 \\
(1.6)\end{array}$ & $\begin{array}{l}59.3 \\
(0.2)\end{array}$ \\
\hline
\end{tabular}


TABLE 3.2

PLASMA FIBRINOGEN, VON WILLEBRAND FACTOR

AND FIBRIN D-DIMER IN CHRONIC ATRIAL FIBRILLATION

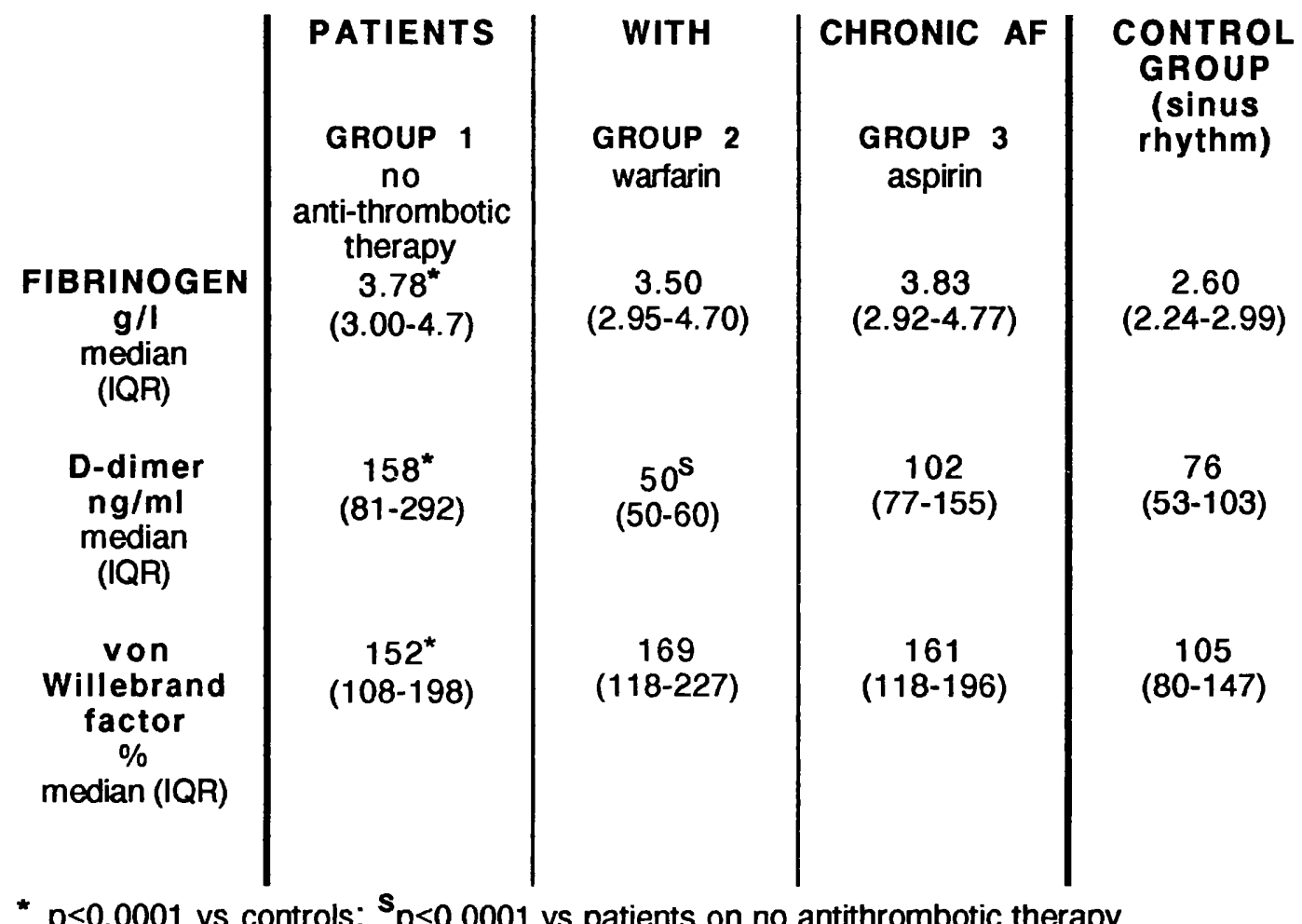

${ }^{*} p<0.0001$ vs controls; ${ }^{s} p<0.0001$ vs patients on no antithrombotic therapy 
TABLE 3.3

\section{ECHOCARDIOGRAPHY IN ATRIAL FIBRILLATION}

\section{LEFT ATRIAL DIMENSION} $\mathrm{mm}$

median (IQR)

LEFT ATRIAL VOLUME $\mathrm{mm}^{3}$ median (IQR) RIGHT
ATRIAL
VOLUME
mm $^{3}$
median (IQR)

\section{LEFT}

VENTRICLE

DIASTOLIC

DIMENSION $\mathrm{cm}$ median (IQR)$$
\text { LEFT }
$$ \\ VENTRICLE \\ fractional \\ shortening $\%$ \\ median (IQR)}

PATIE
GROUP
no
anti-thror

therapy

45.0

(40.1-49.5)

47.6

(34.8-49.7)

40.1

(34.8-49.7)

5.48

(5.0-6.1)

21.8

(16.7-29.0)
WITH

GROUP 2

warfarin

51.0

(43.5-56.0)

63.3

(44.3-84.5)

46.1

(36.4-63.5)

(41.8-49.3)
44.9

(34.6-57.2)

46.8

51.7

(44.0-65.7)

GROUP 3

aspirin

3.37

(3.1-3.7)

29.2

(26.2-31.5)

20.1

(17.2-23.0)

27.2

(19.5-29.9)

CONTROL
GROUP

(sinus

rhythm)

$(n=14)$

5.3

(4.95-6.1)

5.2

(4.9-5.5)

35.3

(30.9-37.3)

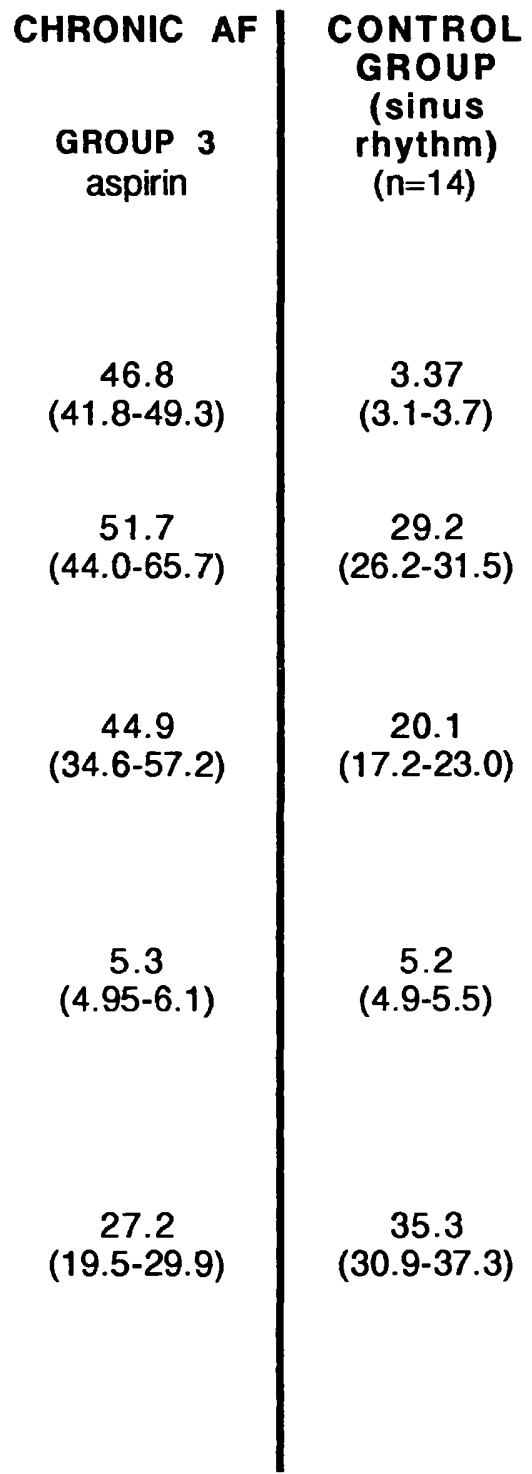


FIGURE 3.4

CHRONIC ATRIAL FIBRILLATION

- SPEARMAN CORRELATION WITH AGE, CARDIAC DIMENSIONS AND VENTRICULAR FUNCTION

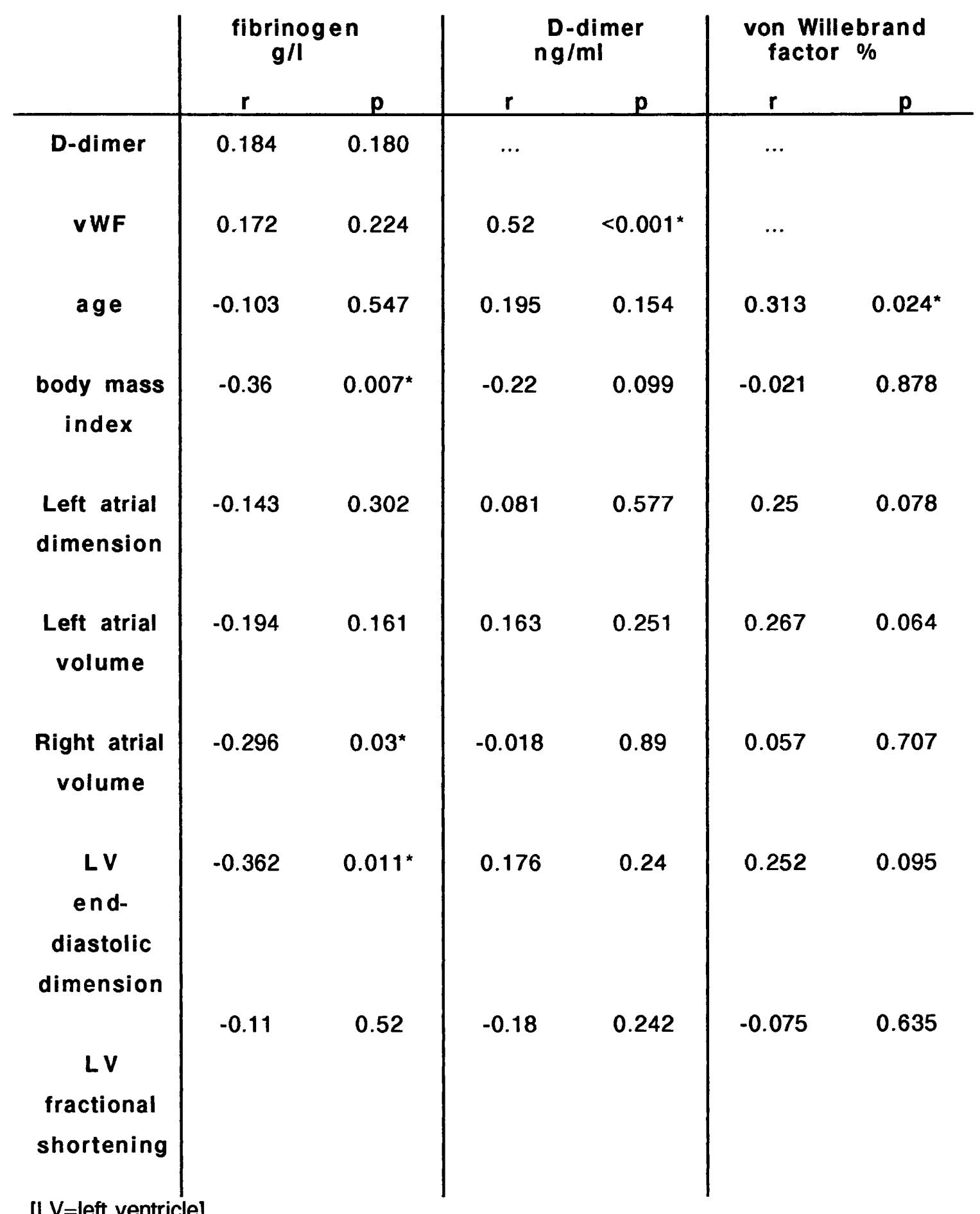

[LV=left ventricle] 
FIGURE 3.5

INTRA- AND BETWEEN-OBSERVER REPEATABILITY FOR 2-

DIMENSIONAL AND M-MODE ECHOCARDIOGRAPHY

(a) intra-observer repeatability (IOR)

\begin{tabular}{|c|c|c|c|c|c|}
\hline MEASUREMENT & $\begin{array}{c}\text { Pearson } \\
r\end{array}$ & $\begin{array}{c}\text { mean } \\
\text { difference }\end{array}$ & $\pm 2 s . d$ & $\begin{array}{c}\text { one } \\
\text { sample } \\
\text { t-test }\end{array}$ & p value \\
\hline \multicolumn{6}{|l|}{ LEFT ATRIUM } \\
\hline D1 & 0.955 & 0.05 & 0.30 & 1.64 & 0.12 \\
\hline $\mathrm{D} 2$ & 0.879 & 0.01 & 0.34 & 0.34 & 0.74 \\
\hline D3 & 0.958 & 0.06 & 0.42 & 1.32 & 0.20 \\
\hline LA VOLUME & 0.872 & 0.88 & 11.4 & 0.73 & 0.48 \\
\hline \multicolumn{6}{|l|}{ RIGHT ATRIUM } \\
\hline $\mathrm{D} 2$ & 0.960 & -0.03 & 0.32 & -0.77 & 0.45 \\
\hline D3 & 0.949 & 0.01 & 0.40 & 0.15 & 0.89 \\
\hline RA VOLUME & 0.973 & -0.39 & 6.28 & -0.56 & 0.58 \\
\hline LEFT & & & & & \\
\hline VENTRICLE & & & & & \\
\hline $\begin{array}{r}\text { end-diastolic } \\
\text { dimension }\end{array}$ & 0.963 & 0 & 0.36 & -0.12 & 0.90 \\
\hline $\begin{array}{r}\text { end-systolic } \\
\text { dimension }\end{array}$ & 0.973 & 0.06 & 0.40 & 1.45 & 0.16 \\
\hline $\begin{array}{r}\text { fractional } \\
\text { shortening }\end{array}$ & 0.918 & -0.01 & 0.06 & -1.82 & 0.09 \\
\hline
\end{tabular}


(b) between-observer repeatability (BOR)

\begin{tabular}{|c|c|c|c|c|c|}
\hline MEASUREMENT & $\begin{array}{c}\text { Pearson } \\
r\end{array}$ & $\begin{array}{c}\text { mean } \\
\text { difference }\end{array}$ & \pm 2 s.d. & $\begin{array}{c}\text { one } \\
\text { sample } \\
\text { t-test T }\end{array}$ & $p$ value \\
\hline \multicolumn{6}{|l|}{ LEFT ATRIUM } \\
\hline D1 & 0.949 & 0.06 & 0.32 & 1.74 & 0.10 \\
\hline D2 & 0.955 & 0.05 & 0.24 & 1.83 & 0.082 \\
\hline D3 & 0.980 & 0.02 & 0.22 & 1.00 & 0.33 \\
\hline LA VOLUME & 0.917 & 0.59 & 9.1 & 0.59 & 0.56 \\
\hline \multicolumn{6}{|l|}{ RIGHT ATRIUM } \\
\hline $\mathrm{D} 2$ & 0.813 & -0.07 & 0.66 & -0.96 & 0.35 \\
\hline D3 & 0.883 & -0.01 & 0.58 & -0.09 & 0.93 \\
\hline RA VOLUME & 0.870 & -1.1 & 13.4 & -0.71 & 0.49 \\
\hline LEFT & & & & & \\
\hline VENTRICLE & & & & & \\
\hline $\begin{array}{r}\text { end-diastolic } \\
\text { dimension }\end{array}$ & 0.955 & 0 & 0.40 & 0.11 & 0.91 \\
\hline $\begin{array}{r}\text { end-systolic } \\
\text { dimension }\end{array}$ & 0.968 & -0.07 & 0.68 & -0.47 & 0.64 \\
\hline $\begin{array}{r}\text { fractional } \\
\text { shortening }\end{array}$ & 0.847 & -0.02 & 0.084 & -1.68 & 0.11 \\
\hline
\end{tabular}

$\mathrm{p}=\mathrm{NS}$ 

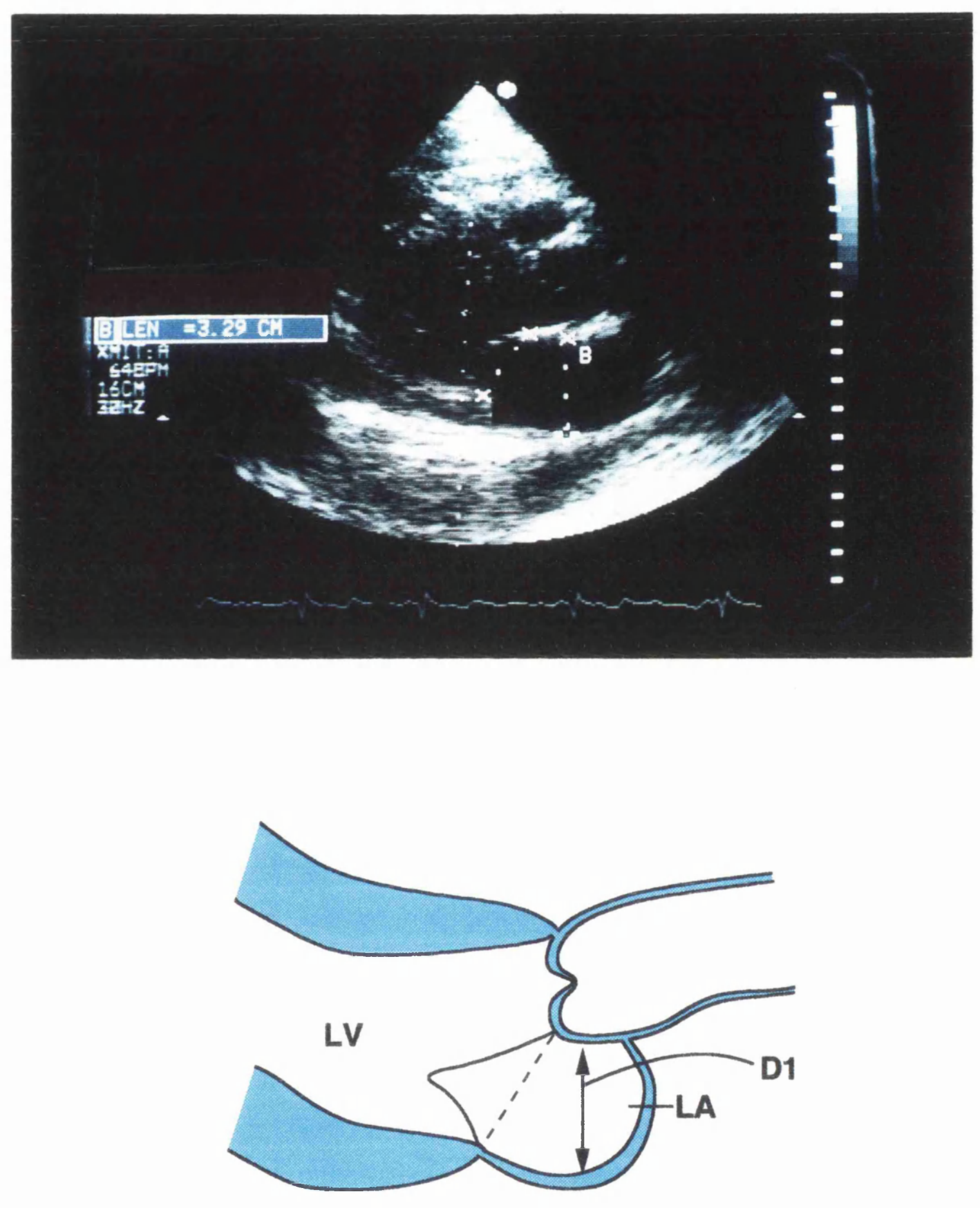

FIGURE 3.1

2 - DIMENSIONAL ECHOCARDIOGRAPHY

Parasternal long axis view with anteroposterior dimension $\left(D_{1}\right)$ for left atrium (LA) [marked ' $B$ ' on picture] 

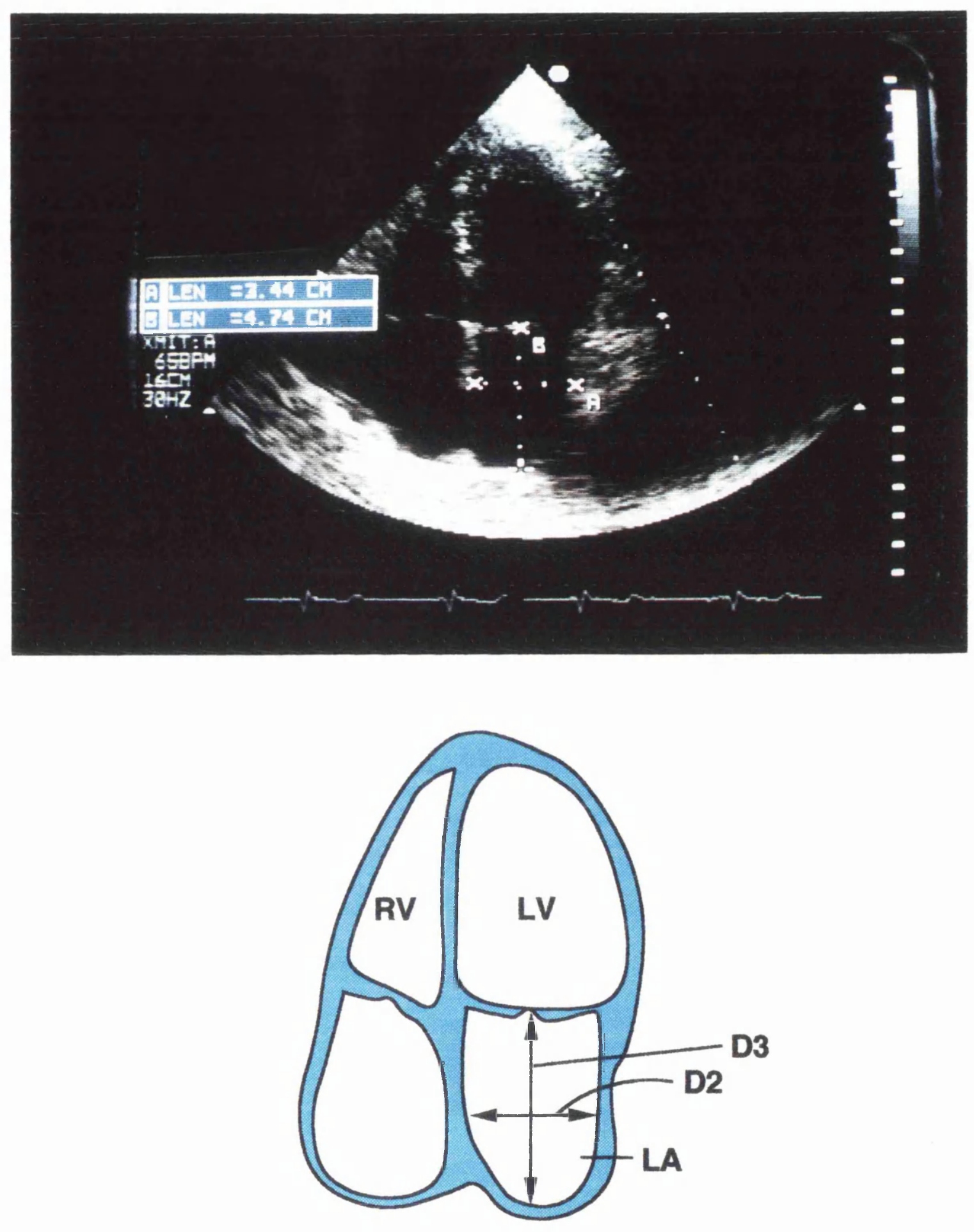

FIGURE 3.2 (a)

\section{2 - DIMENSIONAL ECHOCARDIOGRAPHY}

Apical 4 - chamber view : mediolateral $\left(D_{2}\right)$ and superoinferior $\left(D_{3}\right)$ dimensions for left atrium (LA) [marked ' $A$ ' and ' $B$ ' respectively on picture] 

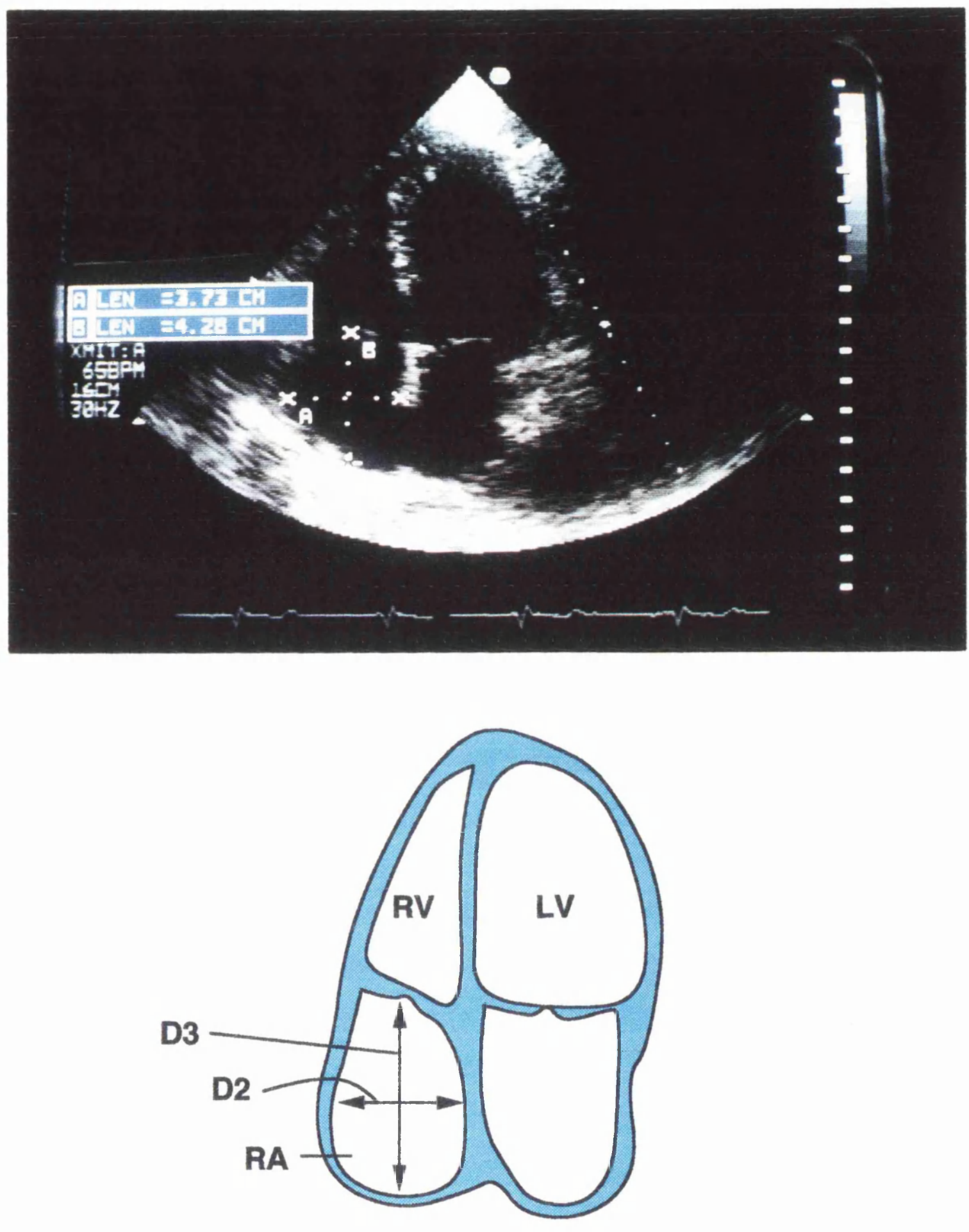

FIGURE 3.2 (b)

\section{2 - DIMENSIONAL ECHOCARDIOGRAPHY}

Apical 4 - chamber view : mediolateral $\left(D_{2}\right)$ and superoinferior $\left(D_{3}\right)$ dimensions for right atrium (RA)

[marked ' $A$ ' and ' $B$ ' on picture] 


\section{ELLIPSOID \\ Length - Diameter}

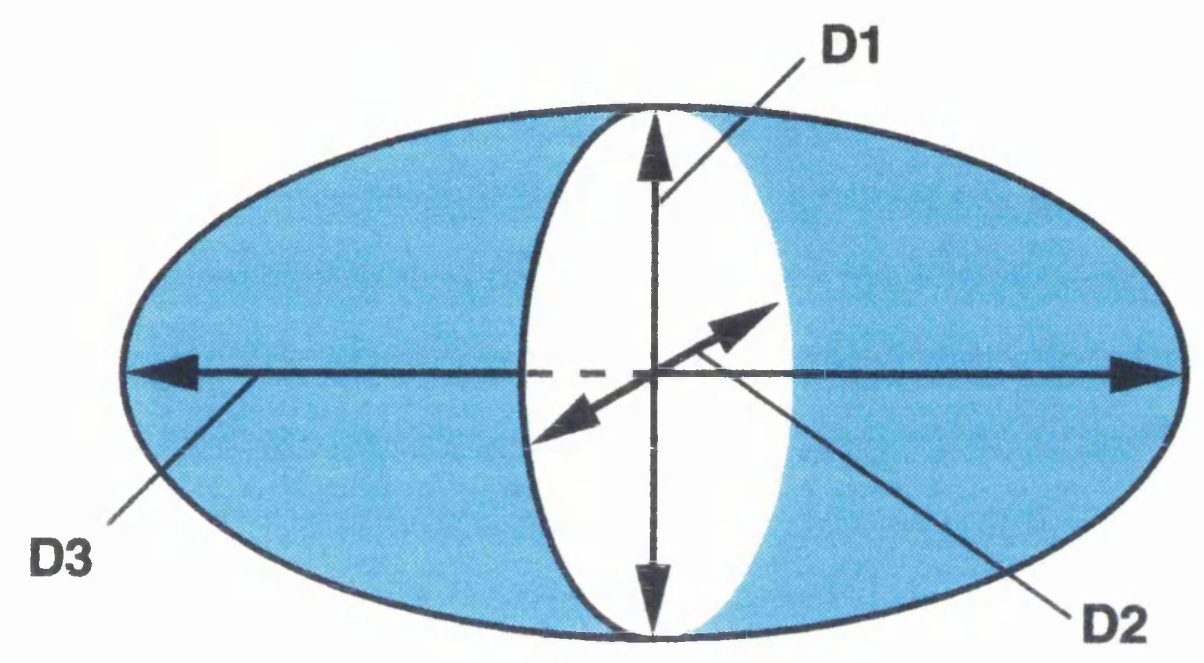

Atrial Volume $=\frac{4 \pi}{3} \times \frac{D 1}{2} \times \frac{D 2}{2} \times \frac{D 3}{2}$

FIGURE 3.3

ELLIPSOID FORMULA USED FOR CALCULATION OF ATRIAL VOLUMES

[for the right atrium, the anteroposterior dimension

$\left(D_{1}\right)$ is assumed equal to the mediolateral dimension $\left(D_{2}\right)$ ] 


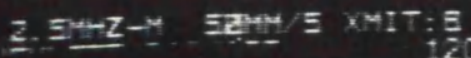

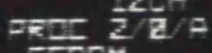
E EZEOE: 14
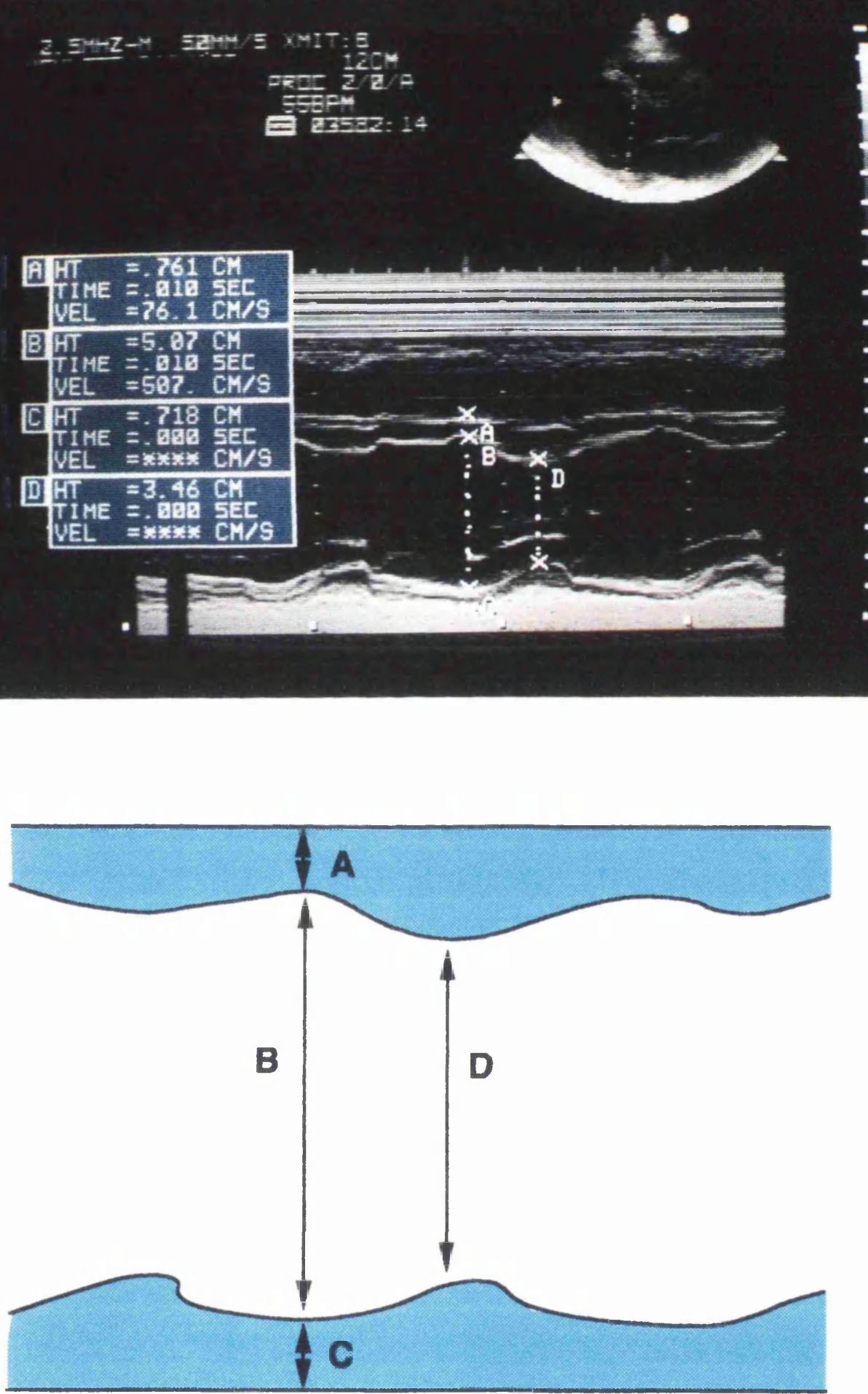

FIGURE 3.4

M-MODE ECHOCARDIOGRAPHY OF LEFT VENTRICLE (LV) with septum (A), LV end-diastolic dimension (B), LV posterior wall (C) and LV end-systolic dimension (D) respectively 


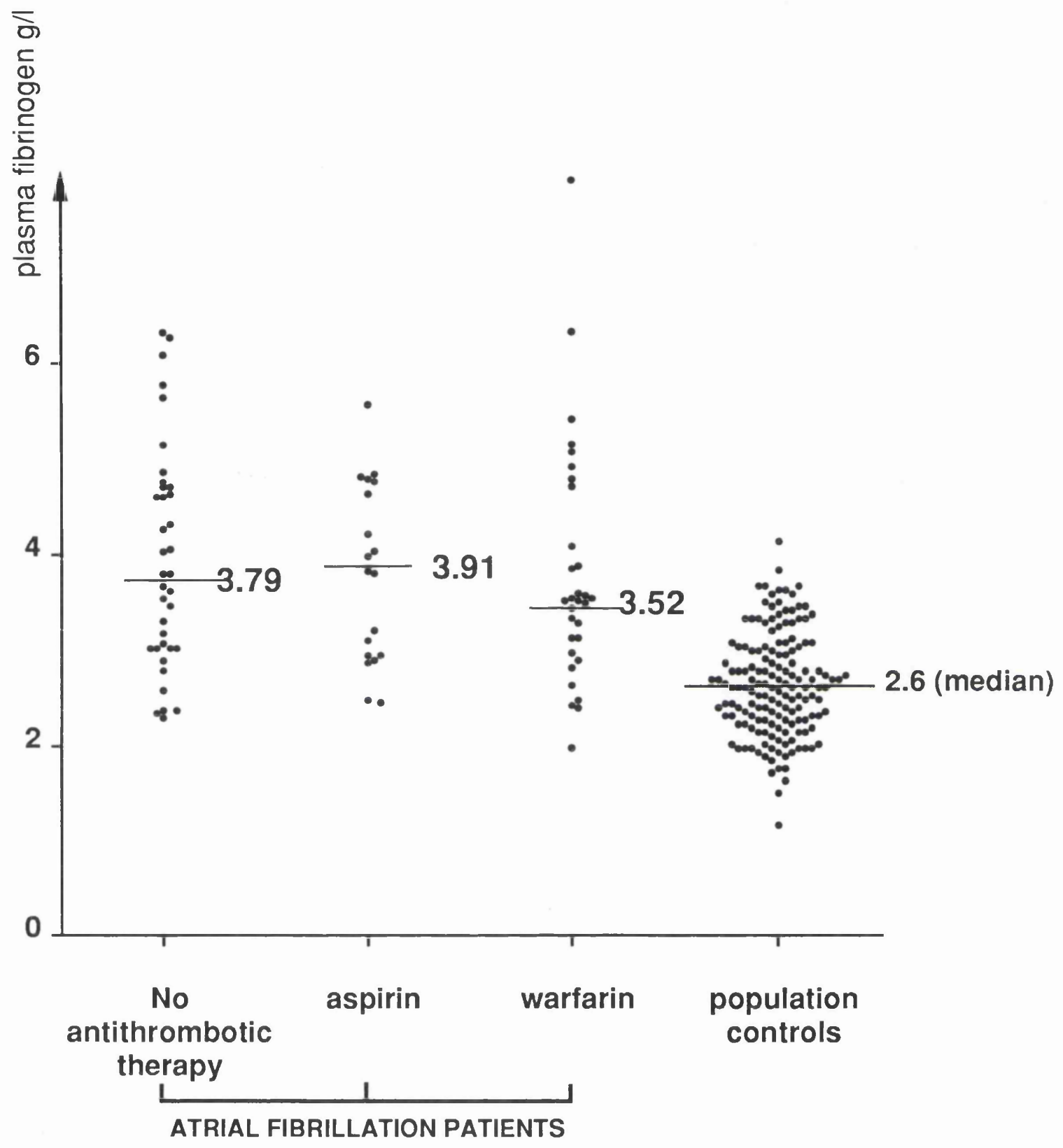

FIGURE 3.5

PLASMA FIBRINOGEN AND CHRONIC ATRIAL FIBRILLATION 


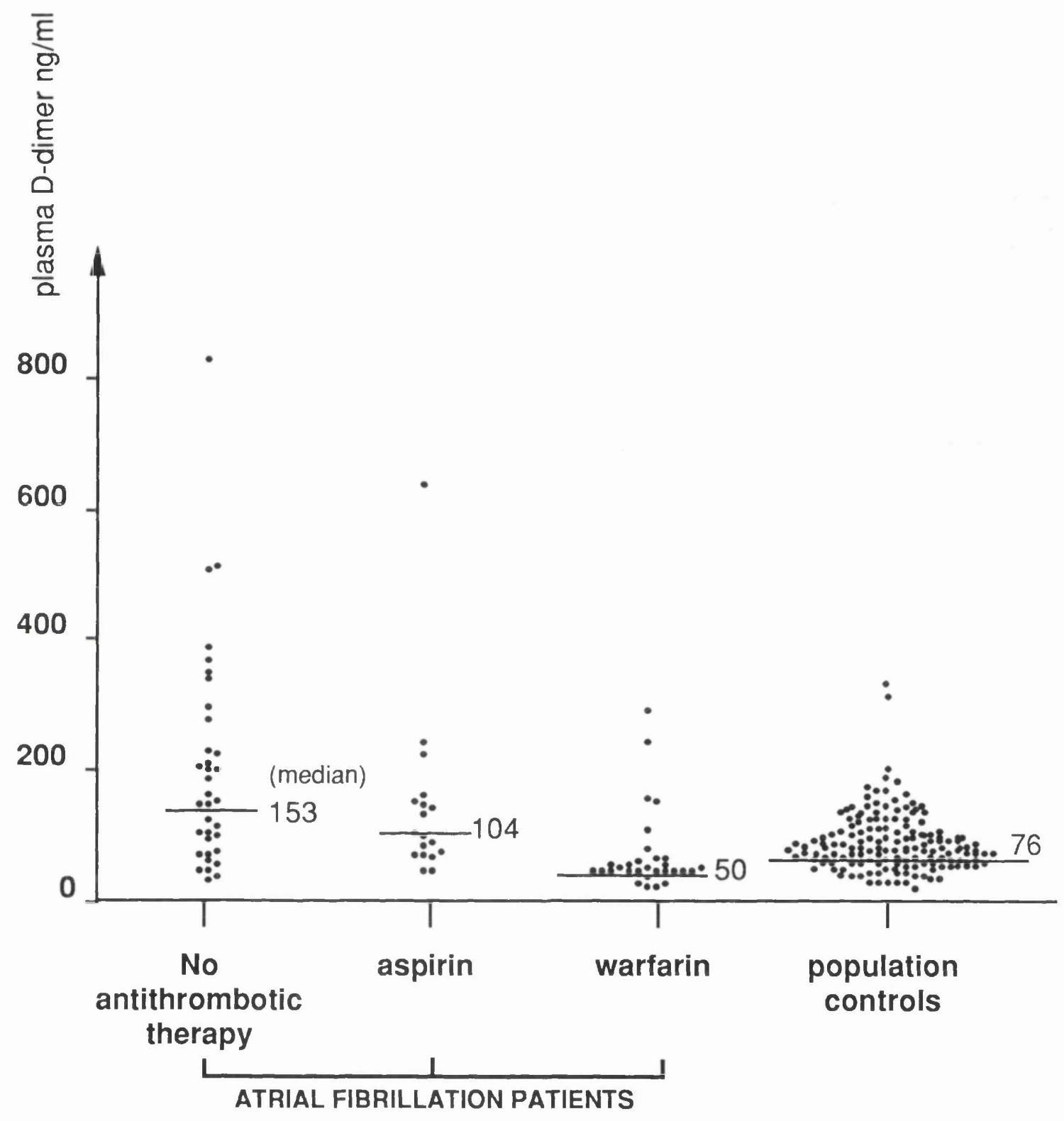

FIGURE 3.6

PLASMA D-DIMER AND CHRONIC ATRIAL FIBRILLATION 


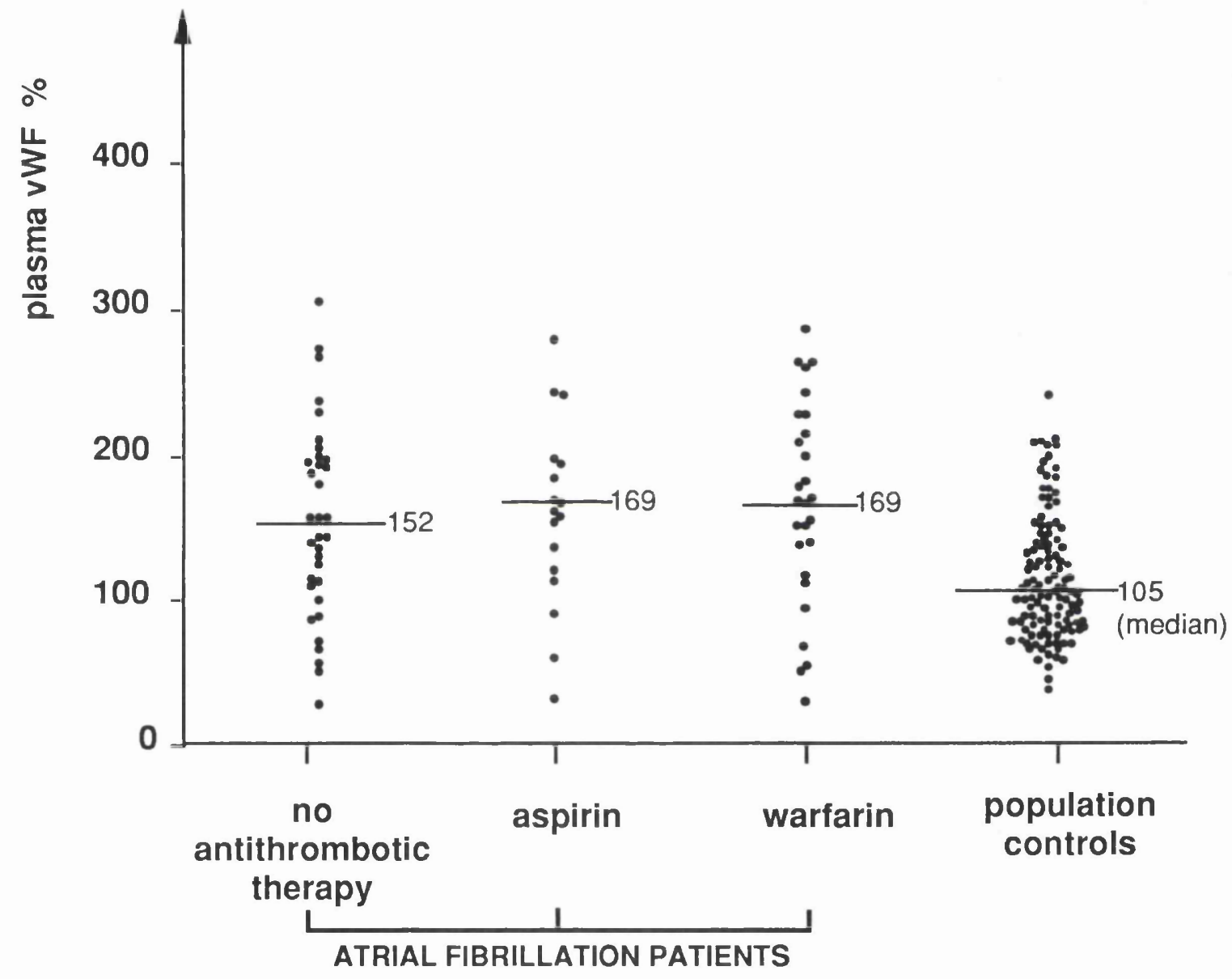

FIGURE 3.7

von WILLEBRAND FACTOR AND CHRONIC ATRIAL FIBRILLATION 
CHAPTER 4

THROMBOGENESIS

AND

CARDIOVERSION OF ATRIAL FIBRILLATION 


\subsection{ANTICOAGULATION AND CARDIOVERSION OF ATRIAL FIBRILLATION}

\section{Introduction}

Atrial fibrillation is an arrhythmia which is unfortunately associated with an increased risk of thromboembolism and haemodynamic disturbance. Rather than to continue in a potentially serious cardiac arrhythmia (associated with an increased mortality, a fivefold risk of stroke and other major embolic events) and the requirement for drugs with potential adverse effects, including oral anticoagulants and antiarrhythmics, cardioversion from atrial fibrillation to sinus rhythm should be considered for suitable patients. The potential benefits of a return to sinus rhythm include an improvement in well-being and exercise capacity (due to the return of atrial filling, and consequent improved cardiac output) (Lipkin et al 1988); avoidance of (potentially dangerous) drug therapy and a reduction in thromboembolic risk.

However, in my survey of management of patients with atrial fibrillation (chapter 2), there was a low proportion of patients considered for cardioversion. This may be a reflection of perceived risk of the procedure, associated thromboembolic risk and the uncertainty of patient selection. In addition, if the procedure is undertaken, there is often uncertainty about the suitable length of anticoagulant therapy required pre- and postcardioversion.

\section{Pathophysiology}

With increasing age and any (progressive) impairment of left ventricular contraction, the dependence on atrial function increases and atrial systole contributes an increasing amount towards overall stroke volume. The ventricular rate is also the most important determinant of overall cardiac output in the physiologically normal heart. However, this observation is not true for very rapid heart rates or in the presence of certain pathology. In atrial fibrillation, the haemodynamic disturbances results essentially from the absence of atrial systole and from the rapidity and irregularity of the ventricular response. A sudden change to a very rapid heart rate may therefore significantly reduce the diastolic filling interval, 
resulting in a dramatic reduction in cardiac output. This will have particular importance in the presence of either valvular stenosis or reduced left ventricular compliance (for example, in left ventricular hypertrophy) (Naito et al 1980). It has also been described that the onset of a rapid ventricular response may also lead to a degree of mitral incompetence thus further reducing forward flow (Naito et al 1980). The deleterious haemodynamic changes of atrial fibrillation have been substantiated by studies showing a significantly improved cardiac output following cardioversion of atrial fibrillation to sinus rhythm (Lipkin et al 1988).

Cardioversion from atrial fibrillation to sinus rhythm may therefore have haemodynamic advantages. It can be performed electrically, by means of a synchronous direct current discharge, or pharmacologically, by the use of a suitable antiarrhythmic drug. Electrical cardioversion works by repolarising the errant atrial conduction, restoring ordered conduction. During the initial asystolic period, the sino-atrial node rapidly resumes its role as cardiac pacemaker. External electrical cardioversion is effective in restoring sinus rhythm in $\mathbf{2 0}$ to $90 \%$ of patients but is highly influenced by the underlying aetiology (Levy 1992, Pritchett 1992). The highest recorded success rates are seen in patients with atrial fibrillation secondary to hyperthyroidism, whilst the lowest rates are seen in patients with severe mitral regurgitation (Levy 1992). In patients who failed external cardioversion, internal cardioversion (using electric shocks delivered within the right atrium) is a specialist technique recently described, with a success rate of $83 \%$ (Levy 1992). Despite the high initial success rates for electrical cardioversion, there is often a high rate of reversion to atrial fibrillation without the concomitant use of an antiarrhythmic agent. For example, in one study, sinus rhythm was maintained in only $69 \%$ and $50 \%$ of patients at 1 and 6 months respectively (Dittrich et al 1989).

An alternative to electrical cardioversion is pharmacological cardioversion. Drugs that are usually employed to maintain sinus rhythm after electrical cardioversion may also be effective for pharmacological cardioversion. In addition, the use of prophylactic antiarrhythmic therapy has been shown to reduce the recurrence of atrial fibrillation postcardioversion (Levy 1992). In general, Class I and Class III antiarrhythmic drugs are the most useful agents for pharmacological cardioversion (Pritchett 1992). Class I drugs commonly in 
use are quinidine, flecainide and propafenone. Quinidine, for example, is very effective in patients with lone atrial fibrillation, but side effects can occur in $22 \%$ (de Nooijer and Sparling 1990). Flecainide and propafenone are also effective, with a rate of successful cardioversion between 25-55\% (Clark and Cotter 1993). Amiodarone, a Class III antiarrhythmic drug, has been shown to be highly effective in cardioversion of atrial fibrillation, even in previously refractory cases (Blevins et al 1987, Noc et al 1990). However, there is concern regarding an increased risk of mortality with the use of antiarrhythmics, for example, the Class 1 antiarrhythmic drugs (Echt et al 1991, Repique et al 1992). It should be noted that digoxin is no better than placebo for the attempted restoration of sinus rhythm (Falk et al 1987).

Another therapeutic consideration during cardioversion (whether pharmacological or electrical) is the use of prophylactic anticoagulation to minimise the risk of thromboembolism following cardioversion. It has been estimated that peripheral emboli may complicate external cardioversion in 1-3\% of cases (Levy 1992). Thromboembolism following chemical cardioversion with amiodarone may well have similar rates (Yapa and Green 1990).

Anticoagulant therapy is therefore advised, despite the absence of prospective randomised trials on the effectiveness of such therapy, in the cardioversion of such patients. However, why these patients should develop thromboembolism is only appreciated by an understanding of the underlying mechanisms promoting thromboembolism during cardioversion.

\section{Mechanism of thromboembolism}

The mechanisms of embolization following cardioversion of atrial fibrillation remain to be clearly defined and various possibilities have been suggested. Firstly, a mechanical explanation for thromboembolism after cardioversion has been suggested. The sudden resumption of mechanical atrial systole may result in the embolisation of any clot formed within the left atrium. However, the minimum time for such thrombi to form in the fibrillating atria is as yet unknown (Mancini and Goldberger 1982), and the mechanisms of thromboembolism are not likely to be simply mechanical. In addition, the time for the return of atrial systole is variable between individual patients, and can take up to 3 weeks post 
cardioversion (Manning et al 1989). It is therefore suggested that anticoagulant therapy postcardioversion should at least be continued until the return of atrial systolic function.

Secondly, abnormalities in haemorheological function and prothrombotic markers intrinsic to atrial fibrillation (as discussed and studied by myself in Chapter 3 of this thesis) may contribute to the thromboembolic risk post-cardioversion. Indeed, patients with chronic atrial fibrillation have abnormalities in plasma fibrinogen and fibrin plasma D-dimer levels, as demonstrated in chapter 3 of this thesis and in other work (Kumagai et al 1990, Gustafsson et al 1990). Atrial fibrillation is also associated with elevated levels of atrial natiuretic peptide, which may contribute to haemoconcentration, a raised haematocrit and subsequent thromboembolism and stroke (Petersen et al 1988). However, if the mechanisms of thrombo-embolism are not simply mechanical but related to an underlying prothrombotic state, serial measurement of suitable markers of thrombogenesis (as undertaken in this thesis) may be useful in determining the duration and intensity of anticoagulant therapy required.

Another factor which may influence the mechanisms of thromboembolism with cardioversion is the left atrial size (Manning et al 1993). Patients with left atrial enlargement, for example, are associated with increased spontaneous echo contrast on transoesophageal echocardiography, suggesting slow or sluggish blood flow within the enlarged atrial cavity (Daniel et al 1988, Manning et al 1993). In addition, spontaneous echo contrast has also been noted during paroxysmal atrial fibrillation, and particularly localised to the left atrial appendage whereas the remainder of the left atrium was clear (Obarski et al 1990). The rheological mechanism for spontaneous echo contrast is thought to involve the presence of fibrinogen, or its products, and red cell aggregation or rouleau formation (Merino et al 1992, Sigel et al 1981), thus providing a 'link' between haemorheological abnormalities and visual demonstration of flow abnormalities with the left atrium, continuously in chronic atrial fibrillation, and intermittently in paroxysmal atrial fibrillation.

Finally, abnormalities in cerebral blood flow may be part of the mechanism for thromboembolic stroke following cardioversion. For example, there appears to be increased cerebral blood flow after cardioversion from atrial fibrillation to normal sinus rhythm, and this 
may predispose to cerebral embolism in the distribution of the middle cerebral artery (Petersen 1990).

\section{The role of anticoagulation}

The role of prophylactic anticoagulation in patients with chronic atrial fibrillation is now established and is discussed and investigated in chapter 10 of this thesis. However, the role of prophylactic anticoagulation to prevent thromboembolism following cardioversion, for patients in atrial fibrillation, has not been as intensely investigated. Consequently, there is a lack of consensus regarding the need for (and the duration of) such therapy before the procedure is undertaken. It is often suggested that the actual conversion to sinus rhythm is the actual risk factor for embolic events rather than the duration of atrial fibrillation. However, other factors may be important, including the haemorheological and prothrombotic abnormalities of atrial fibrillation.

It has been argued that recently formed, poorly adherent thrombus is more likely to dislodge at the time of cardioversion, when compared to older thrombus which is likely to be more firmly adherent by fibroblastic infiltration (Mancini and Goldberger 1982). An estimate of the time required for this infilterative process is approximately 14 days, so anticoagulation for this time period might prevent new clot formation and allow the most recently formed thrombi to become sufficiently adherent (Mancini and Goldberger 1982). However, this time period cannot be precisely defined and is very likely to vary according to the haemodynamic status, atrial size and underlying atrial pathology, together with the effectiveness of anticoagulation.

The role of prophylactic anticoagulation has been clinically examined in several large series. Bjerkelund and Orning (1969) reported a series of 437 patients with atrial arrhythmias in whom electrical cardioversion was attempted on 572 occasions: 228 patients were on long-term anticoagulant therapy, whilst 209 were not given anticoagulants. Thirteen patients (3\%) experienced thromboembolic events, with $2(1.1 \%)$ of the 186 patients in the anticoagulant group, compared to $11(6.8 \%)$ of the 162 patients who were not on anticoagulants and were successfully cardioverted (Bjerkelund and Orning 1969). These 
results were statistically significant, demonstrating that prior anticoagulant therapy was indeed beneficial in attempted cardioversion. Furthermore, the advantageous effect of warfarin occurred despite more patients with congestive heart failure, mitral valve disease and hypertension (factors that increase the risk for thromboembolism) being present in the anticoagulated group. The thromboembolic events that did occur were predominantly noted 1 to 6 days following cardioversion, suggesting a 'high risk' period post-cardioversion. However, this study was not randomised, and as patients were on chronic anticoagulant therapy, the value of short term anticoagulant therapy could not therefore be defined from this study. In addition, this study included patients with atrial flutter and atrial tachycardia (in addition to atrial fibrillation), although the risk of thromboembolism in these subgroups has not been specifically defined (Bjerkelund and Orning 1969).

In another retrospective study of 79 patients who underwent cardioversion, none of the 51 patients who received anticoagulant therapy had an embolic event, whilst 2 of $28(7 \%)$ who were not anticoagulated had an embolic stroke (Weinberg and Mancini 1989). Although the numbers are small, their results do support short-term anticoagulant treatment, especially in high risk patients (who were defined in this study as patients aged >55 years, those with duration of atrial fibrillation $>1$ year, coronary artery disease, cardiomyopathy, or hypertension) (Weinberg and Mancini 1989).

More recently, Arnold et al (1992) retrospectively assessed 454 elective direct current cardioversions performed for atrial fibrillation or atrial flutter over a seven year period. The incidence of embolic complications was $1.32 \%$ ( 6 patients in total). All six had atrial fibrillation, none were on anticoagulants and the duration of atrial fibrillation was $<1$ week in 5 of them (Arnold et al 1992). None of the patients with atrial flutter had thromboembolic events confirming the low thromboembolic risk for cardioversion of this subgroup of patients (Arnold et al 1992).

Whilst the above-mentioned studies were not randomised, the evidence for anticoagulant therapy, prior to cardioversion of atrial fibrillation, appears to be very persuasive. Although anticoagulation is relatively safe, effective anticoagulation is not completely free of a risk of bleeding complications. Clearly, it would therefore be advantageous to avoid 
unnecessary anticoagulation. New developments are the availability of transoesophageal echocardiography, and the availability of plasma prothrombotic markers which may assist management (by avoiding anticoagulation) by detecting 'low risk' patients.

Transoesophageal echocardiography is superior to transthroracic echocardiography in detecting atrial thrombi, particularly those involving the atrial appendage (Manning et al 1993). For example, the sensitivity of transthoracic echocardiography for left atrial body and left atrial appendage thrombi has been reported as $78 \%$ and $35 \%$ respectively (Sherman et al 1990). Although the true sensitivity of transoesophageal echocardiography for detecting atrial thrombi is unknown, but it is likely to be related to the size of the thrombus and the ability to obtain adequate imaging of the atria. It is accepted however that even embolization of small thrombi may cause strokes and considerable morbidity. In a small series, early cardioversion was performed (in 78 patients) without long term oral anticoagulant therapy (but with intravenous heparin for 24 hours), with no excess in embolic events (Manning et al 1993). Larger trials on the use of transoesophageal echocardiography are still required, but for the present, it would probably be a useful investigation in patients in whom anticoagulation is especially hazardous. However, it should be noted that the mechanisms of thromboembolism following cardioversion are unlikely to be simply mechanical (as discussed above), and that the potential for thrombus formation in the initial stages after cardioversion still exists.

Another advance would therefore be the identification of suitable prothrombotic markers, which could help in the risk stratification of patients pre- and postcardioversion. Potential markers of thromboembolic risk include plasma fibrinogen and fibrin D-dimer (the latter as a marker of intravascular clotting), and this aspect is investigated further in this thesis.

Other findings following cardioversion

Following cardioversion, alterations in left atrial size, ventricular function and transient electrocardiographic changes (including conduction disturbances and arrhythmias) may be encountered. This is pertinent as thrombogenesis is related to dilated cardiac 
chambers and ventricular dysfunction; the latter may also be adversely affected by arrhythmias.

There is evidence that cardiac output and exercise capacity may be significantly improved following successful cardioversion to sinus rhythm (Alam and Thorstrand 1992, Atwood et al 1989, Lipkin et al 1988). These benefits may be due to a combination of a reduction in heart rate and the restoration of atrial systole. The former contributes to greater diastolic filling time, coronary flow and reduced myocardial oxygen demand; whilst the latter contributes to increased ventricular filling (Alam and Thorstrand 1992, Atwood et al 1989, Khaja and Parker 1972). Atrial contraction may also contribute to improved mitral and tricuspid valve closure with diminution of valvular insufficiency, resulting in improved cardiac performance (Atwood et al 1989, Khaja and Parker 1972). Rarely, however, acute pulmonary oedema following cardioversion has been described, especially in patients with pre-existing left ventricular impairment (Levy 1992).

Following cardioversion, there is also a reduction in left atrial size with the restoration of atrial systole (Alam and Thorstrand 1992, Manning et al 1989, Van Gelder et al 1991c). Full recovery of atrial mechanical activity may not, however, occur immediately following cardioversion. The resumption of atrial activity may be delayed for days despite the electrocardiogram (ECG) showing immediate onset of sinus P waves (Mancini and Goldberger 1982). In a pulsed Doppler evaluation of atrial mechanical function post-cardioversion, peak A wave velocity and percent atrial contribution to total left ventricular filling did not return to normal until 3 weeks after cardioversion (Manning et al 1989). This finding may have implications for the onset of any clinical benefit (from the restoration of atrial systole) in patients with heart failure, and the delineation of any 'risk period' for thromboembolism (as if atrial systolic function is still suboptimal, patients may continue to be at risk for atrial thrombus). A further study does suggest, however, that the degree of atrial mechanical activity following cardioversion may be very variable and embolic episodes are not necessarily related to this delayed return of atrial mechanical activity following cardioversion (O'Neill et al 1990).

In general, arrhythmias post-cardioversion are due to either inadequate synchronisation or digoxin toxicity (Clark and Cotter 1993). The majority of these abnormal 
rhythms are atrial in origin and trivial (Lown 1967). For example, premature beats and conduction disturbances (first or second degree AV block) are also common (Lown 1967). Ventricular arrhythmias following cardioversion are less common but more serious. The most frequent of these is ventricular fibrillation, which may be induced in about $1 \%$ of cases but is usually reverted by repeat shock (Levy 1992). In addition, postcardioversion transient ST segment elevation may occur, usually related to previous pericardiotomy and age (Van Gelder et al 1991a). These ST segment changes were associated with an unfavourable arrhythmia prognosis, with a lower conversion rate ( $48 \%$ vs $76 \%)$ and diminished long-term maintenance of sinus rhythm (Van Gelder et al 1991a). They do not, however, indicate myocardial infarction, although small rises in creatine kinase may occur with electrical cardioversion. The enzyme usually arises from skeletal muscle and myocardial damage is unlikely, which is confirmed by radionuclide studies (Metcalfe et al 1988).

\section{Post cardioversion management}

Following successful cardioversion to sinus rhythm, it is important to continue with oral anticoagulants and possibly antiarnythmic therapy. The latter is to maintain sinus rhythm and prevent arrhythmia recurrence.

Briefly, current practice favours the maintenance of oral anticoagulation following cardioversion (Clark and Cotter 1993). The risk of embolism continues even after successful cardioversion (Bjerkelund and Orning 1969) as atrial mechanical function may not be restored for several weeks (Mancini and Goldberger 1982). The optimal duration of anticoagulation is, as yet, unclear. Recent recommendations by the American College of Chest Physicians include (i) the administration of warfarin for 3 weeks before elective cardioversion of atrial fibrillation of $\geq 3$ days' duration; (ii) continuation of warfarin therapy for two to four weeks after cardioversion; (iii) administration of intravenous heparin followed by warfarin if cardioversion cannot be postponed for 3 weeks; and (iv) no anticoagulant therapy for atrial fibrillation of < 2 days' duration or for atrial flutter (Dunn et al 1989).

Maintenance of sinus rhythm following cardioversion requires the use of antiarrhythmic drugs such as sotalol, quinidine or amiodarone (Juul-Moller et al 1990). The 
sequential use of different types of antiarrhythmic drugs may improve arrhythmia prognosis in chronic atrial fibrillation or flutter after successful cardioversion (Crijns et al 1991). For example, in a study of 127 patients undergoing cardioversion, serial drug treatment initially with flecainide (Stage I), followed by quinidine or sotalol if recurrence occurred (Stage II), and eventually amiodarone (Stage III), the two-year proportion of arrhythmia-free patients increased from 31\% at Stage I to $63 \%$ at the end of serial treatment (Crijns et al 1991).

\section{Prognosis following cardioversion}

Predictors of refractoriness to cardioversion or unsuccessful maintenance of sinus rhythm include the following: age, duration of arrhythmia, the presence of hypertension, valve disease and other organic heart disease.

An older age, in combination with a large number of previous episodes of arrhythmia, a long previous duration of arrhythmia and the presence of mitral valve disease, were predictive of the unsuccessful maintenance of sinus rhythm (Crijns et al 1991, Dethy et al 1988, Mancini and Goldberger 1982, Van Gelder et al 1991b). In addition, the presence of coronary artery disease, hypertension and organic disease (such as mitral valve disease, aortic stenosis and cardiomyopathy) are detrimental factors for the maintenance of normal sinus rhythm following cardioversion (Mancini and Goldberger 1982, Van Gelder et al 1991c). In a Doppler echocardiographic study, a slow increase ( $<10 \%$ in first 24 hours) in the magnitude of A wave post-cardioversion was also predictive for the recurrence of atrial fibrillation (Dethy et al 1988).

The effects of left atrial size are less certain. In a small study (50 patients), a left atrial dimension of $\geq 45 \mathrm{~mm}$ was important, and had a positive predictive value of $66 \%$ for recurrence of atrial fibrillation (Dethy et al 1988). However, recent studies have, in contrast, demonstrated the converse (that is, left atrial size does not appear to influence outcome following cardioversion) (Brodsky et al 1989, Dalzell et al 1990, Dittrich et al 1989, Van Gelder et al 1991c). In these studies, duration of atrial fibrillation was the most important predictor for outcome following cardioversion. In another study of 100 patients, left atrial volume and other standard echocardiographic parameters (left atrial area, M-mode left atrial dimension, left 
ventricular ejection fraction or left ventricular mass) were not predictive of recurrent atrial fibrillation in patients successfully cardioverted (Truitte et al 1993). These studies suggest therefore that atrial size does not strongly influence outcome of cardioversion and patients should not be excluded on these grounds from consideration of cardioversion (Van Gelder et al 1991c). Even in the presence of a dilated left atrium, long term sinus rhythm (79\% at 12 months) is possible with the use of antiarrhythmic drugs (Brodsky et al 1989).

In general, factors accepted as predicting a high degree of success for cardioversion can therefore be identified, and these are summarised in Table 1 (Alpert et al 1988). Proper selection of patients may therefore improve the cost-benefit ratio of attempted cardioversion.

\section{Conclusion}

Cardioversion to sinus rhythm should be considered for all patients in atrial fibrillation in order to improve cardiac performance and to reduce the long-term risk of thromboembolic complications. Anticoagulant therapy begun before elective cardioversion is advised although data demonstrating the most effective regimen is still lacking. Until then, anticoagulant therapy should ideally be administered two to three weeks prior to and for at least two to three weeks following cardioversion. In emergency cases, anticoagulation should be commenced with full heparinisation together with warfarin on the day of cardioversion and continued for a further four weeks to reduce the possibility of late thromboembolism. 


\section{TABLE 4.1}

\section{FACTORS AFFECTING THE EASE OF CONVERSION FROM ATRIAL FIBRILLATION AND MAINTENANCE OF SINUS RHYTHM}

(adapted from Alpert et al 1988)

Beneficial factors

Short duration of atrial fibrillation

Minimal left atrial dilatation

Large ' $f$ ' waves in the ECG

Absence or mild left ventricular failure

Age $<50$ years
Unfavourable factors

Long duration of atrial fibrillation

Marked left atrial dilatation

Diminutive or absent ' $f$ ' wave in the ECG

Marked left ventricular failure

Age $>50$ years 
4.2 STUdies OF PLASMA FIBRINOGEN AND D-DIMER IN PATIENTS WITH ATRIAL FIBRILLATION : THE EFFECTS OF CARDIOVERSION TO SINUS RHYTHM

Introduction

As patients with atrial fibrillation have a potentially serious cardiac arrhythmia, cardioversion from atrial fibrillation to sinus rhythm is often considered as a therapeutic option. However, if patients with atrial fibrillation are successfully cardioverted to sinus rhythm, their overall thromboembolic risk, as measured by plasma prothrombotic markers (fibrinogen and $D$ dimer), may be significantly altered by the change in cardiac rhythm.

This thesis investigates the thrombogenic potential in atrial fibrillation and as there is clinical evidence that the actual procedure of cardioversion is associated with a significant thromboembolic risk (Arnold et al 1992, Bjerkelund and Orning 1969, Weinberg and Mancini 1989), there may be a potential role for plasma markers (fibrinogen, D-dimer) in assessing this. In particular, the highest thromboembolic risk has been clinically noted in the first six days following cardioversion, suggesting that there may well be a 'high risk' period following this procedure (Bjerkelund and Orning 1969). Prophylactic anticoagulant therapy has therefore been advised to minimise the risk of thromboembolism.

Guidelines on anticoagulation during cardioversion have been recommended by the American College of Chest Physicians, as discussed above (Dunn et al 1989). Despite this, there continues to be wide variation between cardiologists in policy regarding anticoagulation therapy before cardioversion. In particular, there is debate over when (and in whom) to start anticoagulation pre-cardioversion and for how long such therapy should be maintained post-cardioversion.

The aim of this study therefore was to undertake serial samples of plasma fibrinogen and fibrin D-dimer in patients, with atrial fibrillation, who underwent cardioversion in our unit, to determine any change in these plasma markers of thromboembolic risk and define any 'high risk' period for this risk postcardioversion. 
Methods

All patients with atrial fibrillation undergoing cardioversion to sinus rhythm were selected for study. In our Coronary Care Unit, there was a variation in individual policy regarding the use of anticoagulation therapy prior to cardioversion which allowed investigation in different settings. Two different regimens were employed by the four different cardiologists in charge of the Coronary Care Unit for the week: Regime (i) anticoagulation prior to (usually for about two or more weeks), and for two or more months following cardioversion; and regime (ii) intravenous heparin for the day (24 hours) of cardioversion only with no oral anticoagulant therapy pre or post cardioversion. This variation allowed a study of the difference in thrombogenic potential between the two regimes, as assessed by measurement of prothrombotic markers. Patients with atrial flutter were considered at low thromboembolic risk during cardioversion and were excluded from this study.

Prior to the procedure, all patients underwent echocardiography by myself primarily to exclude the presence of intracardiac thrombus and to determine cardiac function. All patients underwent either electrical or pharmacological cardioversion, decided on clinical grounds by the consultant in charge. Electrical cardioversion was performed following induction of a light general anaesthesia (preoxygenation and intravenous etomidate $\mathbf{3 0 0}$ $\mathrm{mcg} / \mathrm{kg}$ in aliqouts to achieve anaesthesia) with an initial synchronised direct current shock at 50 Joules. In no instance was endotracheal intubation necessary. If unsuccessful, the procedure was repeated with successively higher energies (100 Joules, $200 \mathrm{~J}, 300 \mathrm{~J}, 360 \mathrm{~J}$ ) up to a maximum of two 360 Joule shocks. If pharmacological cardioversion was attempted, intravenous amiodarone (5mg/kg over 30 minutes, followed by an infusion of $1.2 \mathrm{~g}$ over 24 hours) was used in all cases. All patients underwent monitoring in the coronary care unit during the procedure.

As previously described (in chapter 3), blood samples were taken atraumatically and placed in citrate tubes (trisodium citrate $0.11 \mathrm{M}, 9: 1 \mathrm{v}: \mathrm{v}$ ). The plasma was immediately separated and frozen at $-40^{\circ} \mathrm{C}$. Blood samples were taken pre-cardioversion, and also at 3 days, 7 days and 14 days post cardioversion and assayed for fibrinogen and Ddimer, as previously described. In patients on warfarin therapy, a Thrombotest or INR 
(International Normalised Ratio) was measured to ensure a satisfactory level of anticoagulation. Initial plasma levels of fibrinogen and D-dimer of patients in Group I (no warfarin) were compared with normal population values derived from 158 patients in sinus rhythm sampled from a random population survey (2nd North Glasgow MONICA study), as in chapter 3.

All the values of the blood measurements were expressed as median \pm interquartile range (IQR). Statistical comparison was performed using the Mann-Whitney test and two-sample paired Wilcoxon test for non-parametric distributions. A probability of $\leq 0.05$ was considered statistically significant for paired comparisons.

Results

A total of 19 patients (14 male, 5 female) in atrial fibrillation were admitted to our Coronary Care Unit for elective cardioversion over a one year period: 7 patients (5 male, 2 female; mean age 60.3 years, range 50 to 72 years, s.e.m. 3.71) were on no anticoagulant therapy (Group I), whilst 12 patients (9 male, 3 female; mean age 60.2 years, range 41 to 72 years, s.e.m. 2.70) had anticoagulant therapy pre- and post-cardioversion (Group II).

Echocardiography was performed in all patients. There was no evidence of intracardiac thrombus in any patient. Cardioversion was attempted in all patients - in Group I, 3 patients underwent electrical cardioversion, whilst 4 underwent pharmacological cardioversion; in Group II, 8 underwent electrical cardioversion, whilst 4 underwent pharmacological cardioversion. Cardioversion was considered unsuccessful in 4 cases (3 male, 1 female) from Group II with a reversion to atrial fibrillation within 24 hours whilst as an inpatient. These patients were excluded from further analysis, which was therefore performed on 8 patients in Group II ( 6 male, 2 female; mean age 64.6 years, range 54-72 years, s.e.m. 2.31). Patient characteristics are summarised in Table 4.2. One patient from Group II reverted to atrial fibrillation at the two-week follow-up visit - no blood sample was therefore taken at this visit. There were no instances of systemic thromboembolism in any patient. 
In Group I (no warfarin), pre-cardioversion plasma fibrinogen and fibrin D-dimer levels were significantly elevated when compared to population controls in sinus rhythm (point estimate of difference between medians; for plasma fibrinogen $0.59 \mathrm{~g} / \mathrm{l} ; 95 \% \mathrm{C} . \mathrm{I} .0 .06$ to 1.25 , Mann-Whitney test $p=0.03$; for plasma D-dimer $98 \mathrm{ng} / \mathrm{ml} ; 95 \%$ C.I. 15 to 150 , $p=0.01$ ) (Tables 4.3, 4.4). In Group II (warfarin), pre-cardioversion plasma fibrinogen was not significantly different when compared to similar patients in Group I (no warfarin) (point estimate of difference between medians $0.58 \mathrm{~g} / \mathrm{l} ; 95 \%$ C.I. -1.42 to $0.59, \mathrm{p}=\mathrm{NS}$ ) (Table 4.3). However, patients in Group I (no warfarin) had increased plasma D-dimer levels when compared to similar patients in Group II (warfarin therapy) (point estimate of difference between medians 109 ng/ml; 95\% C.I. 13 to $193, p=0.03$ ) (Table 4.4).

In Group I (no warfarin), there were no significant changes in plasma fibrinogen at Day 3, Day 7 or Day 14 ( $p=N S)$ following cardioversion (Table 4.3, Figure 4.1). Sequential measurements of plasma fibrin D-dimer following cardioversion also demonstrated no significant differences at Day 3 or Day 7 , but there was however a significant reduction of median plasma D-dimer at Day $14(200 \mathrm{ng} / \mathrm{ml}$ vs $42 \mathrm{ng} / \mathrm{ml}$, paired Wilcoxon test $\mathrm{p}=0.02)$ (Table 4.4, Figure 4.1). This was a median reduction of plasma D-dimer by $69 \%$ ( $95 \%$ c.i. $31 \%$ to $79 \%)$ in these patients.

In Group II there were no significant differences in median plasma fibrinogen or fibrin D-dimer at Day 3, day 7 or Day 14 post cardioversion (Tables 4.3, 4.4; Figure 4.2).

When patients from Group I and II were analysed together ( $n=15$; mean age 62.6; range 50-72; s.e.m. 2.1) they had an elevated median plasma fibrinogen when compared to population controls (median difference $0.89 \mathrm{~g} / \mathrm{l} ; 95 \%$ c.i. 0.48 to 1.29 , $p=0.0001$ ). However there was no significant difference between plasma fibrinogen at Day 14 post cardioversion when compared to pre-cardioversion levels (3.56 vs 3.69g/l; paired Wilcoxon $\mathrm{p}=0.68)$.

Discussion

The mechanisms by which many patients with atrial fibrillation develop either intracardiac thrombus or systemic embolism following cardioversion remain uncertain. For 
example, emboli may be due to pre-existing thrombus, which is dislodged by the mechanical effect of a change in rhythm during cardioversion. If these mechanisms are not simply mechanical (which is likely) then merely cardioverting a subject back into sinus rhythm may not immediately reduce the risk until other associated factors (for example, plasma fibrinogen and other rheological factors) have been substantially normalised. This is particularly pertinent as normal haemodynamics would be expected to return (with 'normal' flow characteristics) as atrial systole eventually resumes following cardioversion. However, this may be an oversimplification as in the early post-cardioversion period, atrial asystole may still be present, leading to intra-atrial stasis, thus promoting thrombogenesis.

In this study, no significant differences in plasma fibrinogen levels are demonstrated, however, when pre-cardioversion levels are compared to levels at Day 14 . It is long recognised that there is individual variation in the return of atrial systolic function, which can take up to three weeks to return fully following cardioversion (Manning et al 1989). During the period before the return of atrial systolic function, the patient may still be at risk of forming intracardiac thrombus. This 'delay' may therefore account for the lack of any significant reduction in plasma fibrinogen levels in these patients at two weeks following cardioversion. As plasma fibrinogen is associated with increased cardiovascular and thromboembolic risk, my results therefore suggest that caution should be employed in withholding anticoagulant therapy in patients being considered for cardioversion. In fact, this study substantiates the feeling that anticoagulation should be continued for some time after cardioversion in all patients. Another argument for prolonged anticoagulation is the fact that several patients will revert back to atrial fibrillation following cardioversion.

This study however demonstrates a significant reduction in plasma fibrin Ddimer following cardioversion of atrial fibrillation to sinus rhythm in patients who were not on any oral anticoagulant therapy. As plasma fibrin D-dimer is a marker of ongoing fibrin turnover and intravascular thrombus formation, this study demonstrates that these processes are reduced in patients following cardioversion (at two weeks) to sinus rhythm. Although clinical studies have demonstrated a rise in thromboembolic risk in the first six days after cardioversion (Bjerkelund and Orning 1969) there was no significant elevation in plasma fibrin 
D-dimer at Day 3 and Day 7 following cardioversion in this study. This suggests that any thromboembolic event (as suggested in clinical studies) is likely to be due to preformed thrombus rather than formation of actual new thrombus. Longer term studies are possibly therefore required to assess any further changes in plasma fibrinogen and D-dimer following cardioversion in patients who are not anticoagulated. As there is now increasing evidence for the routine use of anticoagulation therapy pre- and post-cardioversion, there remains an ethical consideration if warfarin is to be withheld from these patients in any further study. (Following the initial results of this study, new guidelines for full anticoagulation pre- and post cardioversion have since been implemented in our coronary care unit).

In patients taking warfarin therapy before and after cardioversion, there were no significant differences in plasma fibrinogen or D-dimer following cardioversion to sinus rhythm. Warfarin therapy therefore beneficially reduces the processes of fibrin turnover and thrombogenesis (as indicated by plasma D-dimer levels) in patients with atrial fibrillation resulting in a 'low' thrombogenic state prior to cardioversion. These results are also consistent with my findings in chapter 3 demonstrating higher plasma fibrin D-dimer in patients with atrial fibrillation when compared to similar patients in sinus rhythm.

This study therefore gives additional evidence to the long term advantages in reducing thromboembolic risk by the cardioversion from atrial fibrillation to sinus rhythm. This study also reinforces the view that warfarin therapy prior to cardioversion would be advantageous in minimising the potential thromboembolic risk. 


\section{TABLE 4.2}

\section{CARDIOVERSION OF ATRIAL FIBRILLATION - PATIENT CHARACTERISTICS}

$\begin{array}{ccc}\text { GROUP I } & \text { GROUP II } & \text { WHOLE GROUP } \\ \text { no warfarin } & \text { anticoagulation }\end{array}$

n

age

mean (s.e.m.)

$\operatorname{sex}(M: F)$

body mass

index

$(\mathrm{kg} / \mathrm{m} 2)$

median (IQR)

left atrial

dimension

$\mathrm{mm}$

median (IQR)

left atrial volume

(ellipsoid)

$\mathrm{mm} 3$

median (IQR)

LV fractional

23.8

(22.0-29.5)

4.24

(3.64-4.34)

41.4

(24.3-49.3)

shortening
8

64.6

(2.3)

$6: 2$

37.8

(22.8-38.8)
15

62.6

(2.12)

11:4

36.9

(35.7-38.8)

4.36

4.28

(4.15-4.7)

(4.06-4.5)

40.5

40.9

(33.9-47.5)

(32.4-47.6)

27.2

24.0

$\%$

(20.4-33.8)

(22.0-33.4)

median (IQR)

[only successfully cardioverted patients are tabulated] 


\section{TABLE 4.3 \\ PLASMA FIBRINOGEN LEVELS AND CARDIOVERSION OF ATRIAL FIBRILLATION}

\begin{tabular}{ccccc} 
& \multicolumn{2}{c}{ median (IQR) $\mathbf{g} / \mathbf{l}$} & \\
& Precardioversion & Day 3 & Day 7 & Day 14 \\
\hline GROUP I & 3.0 & 3.41 & 3.07 & 3.18 \\
& $(2.63-3.97)$ & $(2.58-4.75)$ & $(2.63-3.5)$ & $(2.75-4.2)$ \\
GROUP II & 3.62 & 3.93 & 4.06 & 3.58 \\
& $(3.41-4.08)$ & $(3.23-4.29)$ & $(3.03-4.57)$ & $(3.42-4.04)$ \\
\hline WHOLE & 3.60 & 3.69 & 3.50 & 3.56 \\
GROUP & $(2.83-4.05)$ & $(3.16-4.350$ & $(2.76-4.22)$ & $(2.96-4.1)$
\end{tabular}

Median plasma Fibrinogen values for population controls $(n=158): 2.6 \mathrm{~g} / \mathrm{l}$ (IQR 2.24-2.99) $(p=N S)$

\section{TABLE 4.4 \\ PLASMA D-DIMER LEVELS AND CARDIOVERSION OF ATRIAL FIBRILLATION}

\begin{tabular}{ccccc} 
& \multicolumn{3}{c}{ median (IQR) $\mathrm{ng} / \mathrm{mI}$} \\
Precardioversion & Day 3 & Day 7 & Day 14 \\
GROUP I & 200 & 188 & 142 & $52^{\star}$ \\
& $(63-223)$ & $(56-142)$ & $(49-208)$ & $(30-74)$ \\
GROUP II & 47 & 48 & 62 & 47 \\
& $(33-99)$ & $(32-75)$ & $(32-84)$ & $(30-111)$
\end{tabular}

Median plasma D-Dimer values for population controls $(n=158): 76 \mathrm{mg} / \mathrm{dl}$ (IQR 53-103)

( ${ }^{*} p<0.05$ when compared to pre-cardioversion levels) 


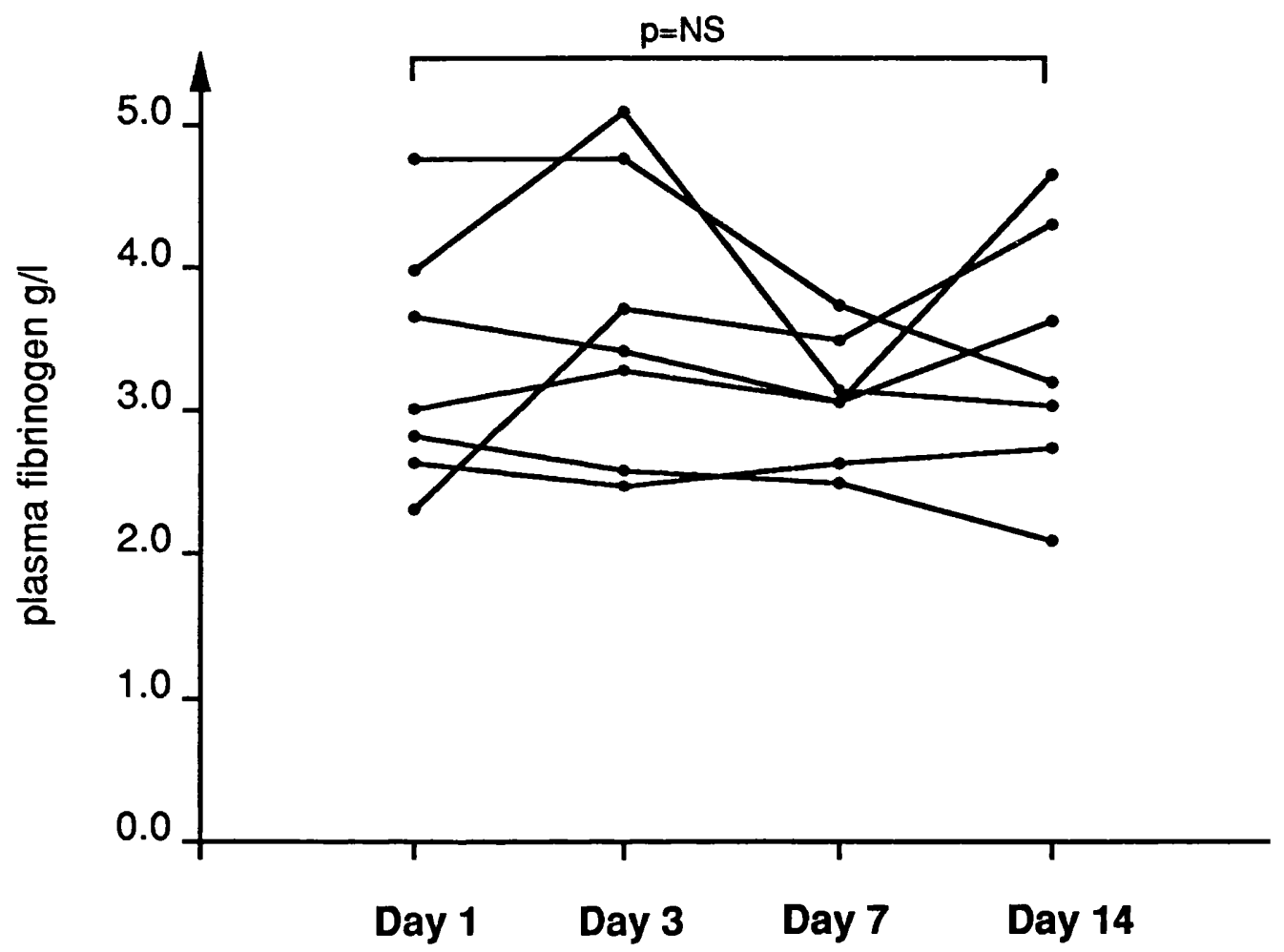

FIGURE 4.1 (a)

CARDIOVERSION AND PLASMA FIBRINOGEN IN ATRIAL FIBRILLATION

(no warfarin group) 


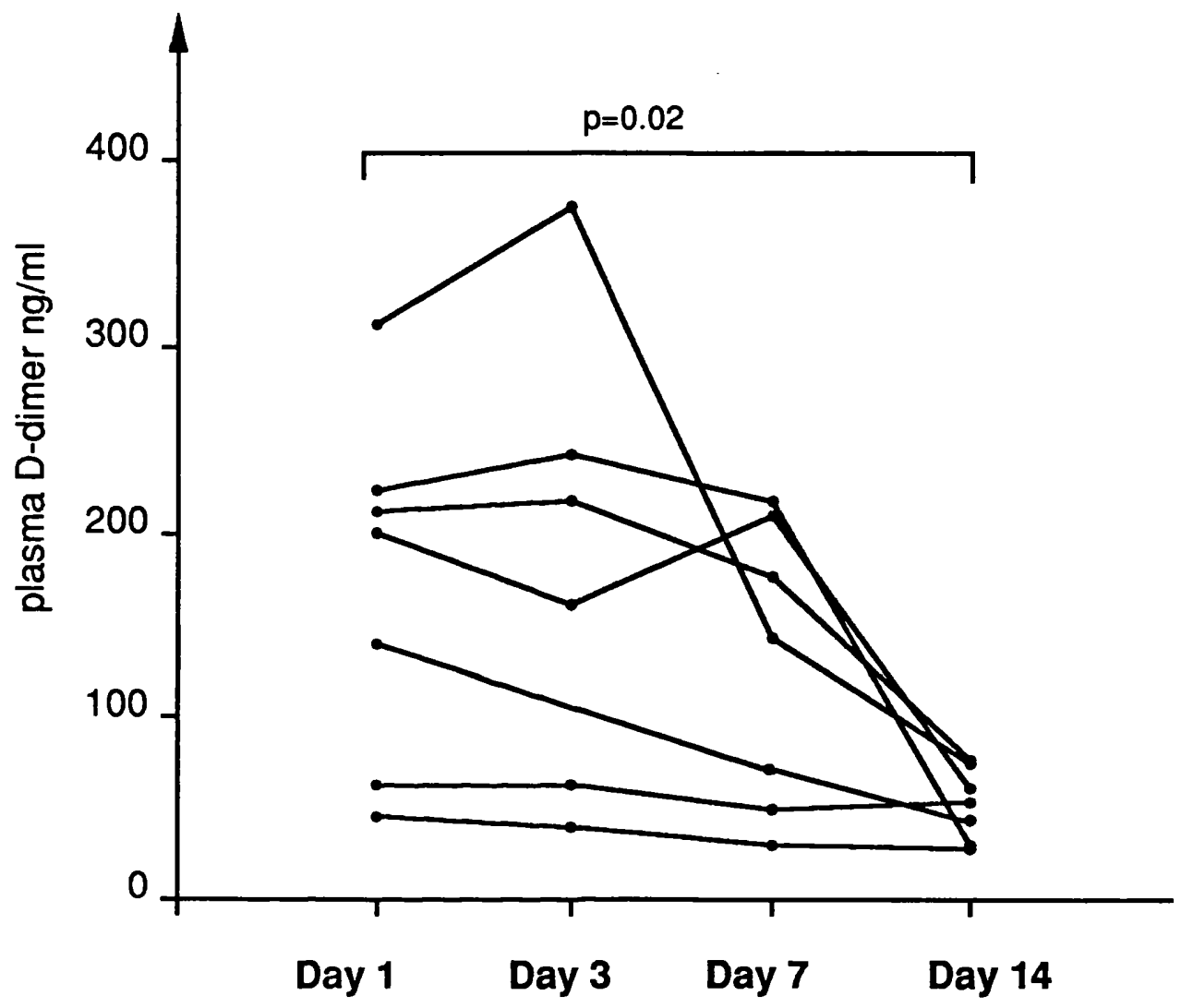

FIGURE 4.1 (b)

CARDIOVERSION AND PLASMA D-DIMER IN ATRIAL FIBRILLATION

(no warfarin group) 


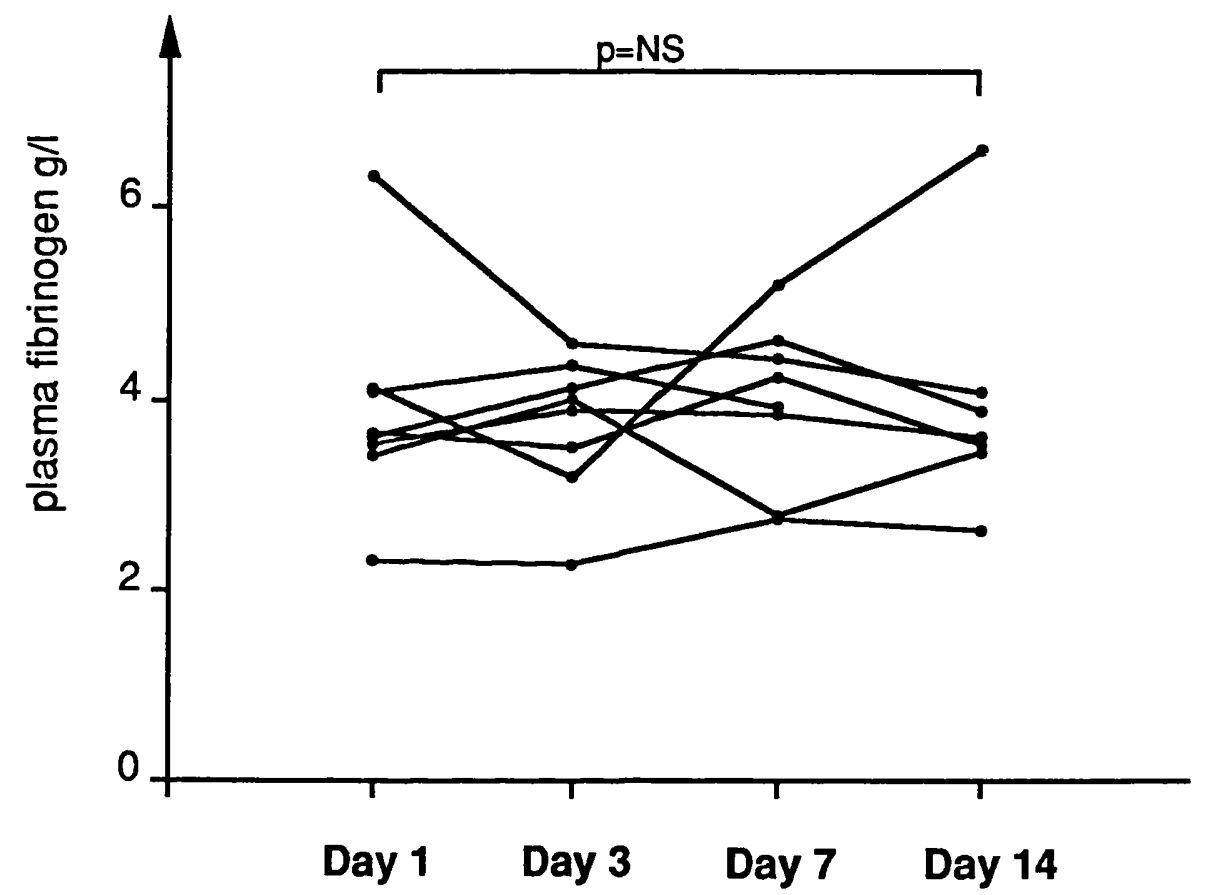

FIGURE 4.2 (a)

CARDIOVERSION AND PLASMA FIBRINOGEN IN ATRIAL FIBRILLATION

(warfarin group) 


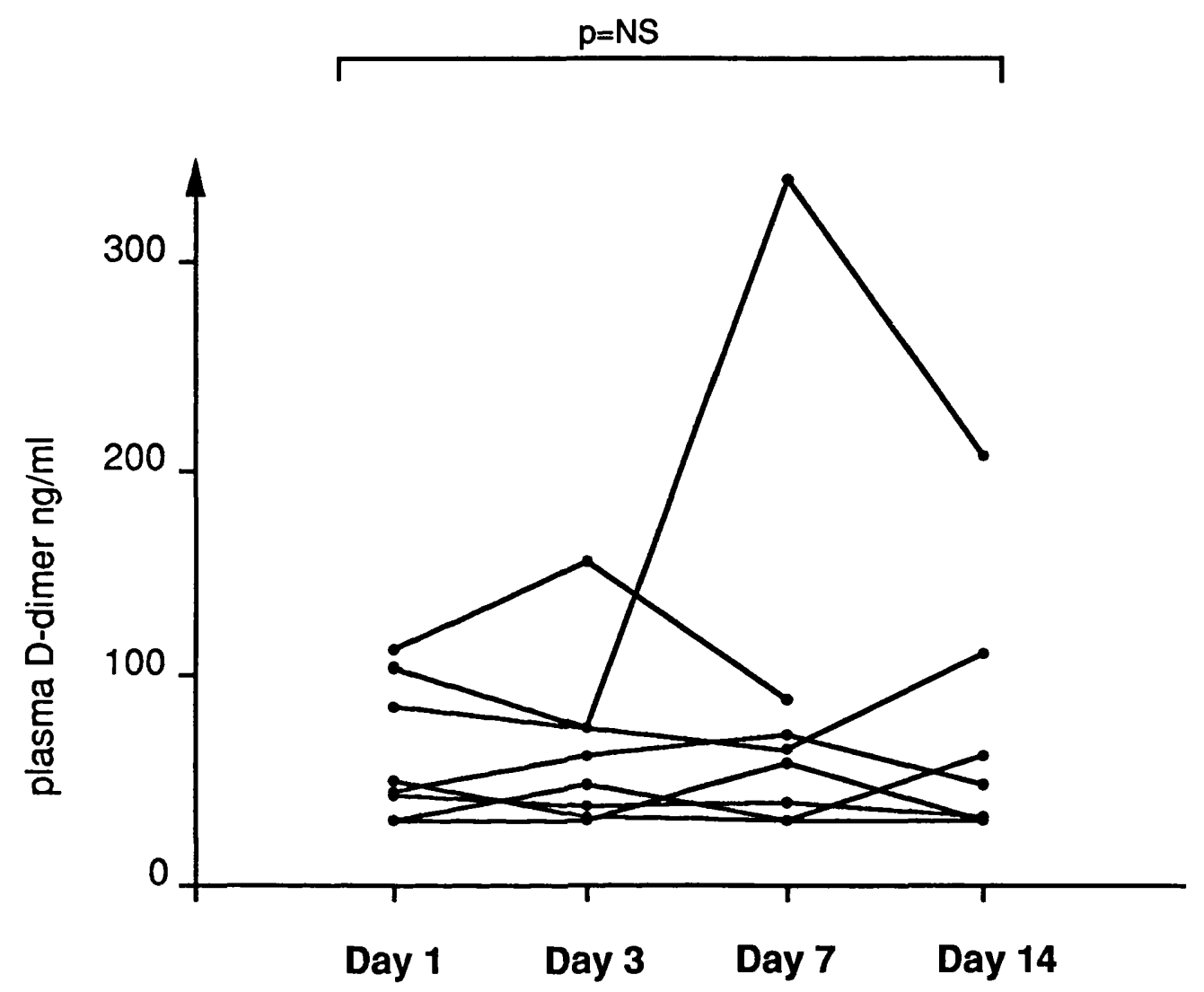

FIGURE 4.2 (b)

CARDIOVERSION AND PLASMA D-DIMER IN

ATRIAL FIBRILLATION

(warfarin group) 
CHAPTER 5

THROMBOGENESIS IN PAROXYSMAL ATRIAL FIBRILLATION 
Introduction

The importance of paroxysmal atrial fibrillation as a thromboembolic risk factor has been relatively neglected. In addition, the effective use of antiarrhythmic therapy and the role of prophylactic anticoagulation therapy in this condition remain controversial areas.

As the management of this common condition is often difficult, I shall initially review the current management of paroxysmal atrial fibrillation and this will be followed by a study of thrombogenesis in such patients.

\subsection{A REVIEW OF THE MANAGEMENT OF PAROXYSMAL ATRIAL FIBRILLATION}

\section{Introduction}

Patients with atrial fibrillation can be divided into a group permanently in atrial fibrillation ("chronic atrial fibrillation") and a group with sinus rhythm punctuated by attacks of atrial fibrillation ("paroxysmal atrial fibrillation"). The two groups overlap, however, as many patients with chronic atrial fibrillation first present with paroxysmal atrial fibrillation (Kopecky 1987) and patients with paroxysmal atrial fibrillation may occasionally revert to sinus rhythm (Chevalier 1979, Olsson et al 1980). In one series, $25 \%$ of patients with paroxysmal atrial fibrillation and nomal hearts progressed to chronic atrial fibrillation within a year (Takahashi et al 1981).

Paroxysmal atrial fibrillation, however, remains one of the commonest arrhythmias, one of the least understood and one of the most difficult to treat. The condition is more prevalent in the elderly and can be associated with underlying structural heart disease including mitral valve disease or congenital heart disease, ischaemic heart disease, sick sinus syndrome, hypertension or thyrotoxicosis, in a similar way to chronic atrial fibrillation. There is a tendency therefore to treat this paroxysmal atrial fibrillation in a similar fashion to chronic atrial fibrillation. However, the pathophysiology of paroxysmal atrial fibrillation is different, and drugs such as digoxin, which are commonly used in chronic atrial fibrillation, are of limited value in paroxysmal atrial fibrillation. In addition, a broad range of clinical features may occur and even in the asymptomatic patient, there 
is concern about the risk of serious thromboembolic events. In practice, effective control of paroxysms is often difficult and the role of anticoagulation remains controversial.

\section{Pathophysiology and clinical features}

The haemodynamic disturbance of atrial fibrillation results essentially from the absence of atrial systole ('atrial kick') and from the rapidity and irregularity of the ventricular response, with a consequent loss of cardiac output. The presence of atrial fibrillation is associated with a fall in cardiac stroke output of approximately $10 \%$ in normal individuals (Broch and Müller 1957). This is more important with increasing age and/or progressive impairment of left ventricular contraction, as atrial systole contributes an increasing amount towards overall stroke volume. Loss of this sequential atrio-ventricular contraction mechanism, in association with a rapid and irregular ventricular response during a paroxysm of atrial fibrillation, reduces the diastolic filling interval and can therefore lead to a dramatic reduction in cardiac output. This is substantiated by evidence of a significantly improved cardiac output following cardioversion of atrial fibrillation to sinus rhythm (Lipkin et al 1988).

For these reasons, many patients, following the onset of a paroxysm of atrial fibrillation, experience not only palpitation but often a combination of presyncope, syncope, fatigue, dyspnoea and lethargy. Occasionally pulmonary oedema, angina or even cerebral impairment may be precipitated. Indeed, paroxysmal atrial fibrillation may be associated with considerably more disabling symptoms than chronic atrial fibrillation, due to the very rapid heart rates during the arrhythmia.

\section{Management}

\section{Arrhythmia control}

One of the most important principles of arrhythmia control is to treat only symptomatic patients. If a patient is experiencing only mild and infrequent symptoms it is advisable to try and avoid antiarrhythmic drug therapy if at all possible (Singh et al 1991). This 
management stratagem is justified by evidence of substantial morbidity associated with antiarrhythmic therapy and the concern over worsened long-term prognosis, particularly with Class I agents (Pritchett 1992, Singh et al 1991). General measures should always be contemplated, for example, withdrawal of caffeine or alcohol, stress counselling and consideration of conditions which may benefit from atrial pacing, for example, sick sinus syndrome. It is also important to distinguish between atrial fibrillation and atrial flutter as there are important management differences between these two conditions.

\section{Digoxin}

Of the antiarrhythmic drugs in use, digoxin continues to be commonly prescribed in paroxysmal atrial fibrillation, in an attempt to both suppress the arrhythmia and to control the initial heart rate if any such paroxysms occur (Falk and Leavitt 1991). Although it remains an effective treatment for chronic atrial fibrillation, its use in paroxysmal atrial fibrillation is more controversial and may even be detrimental (Falk and Leavitt 1991, Galun et al 1991, Rawles et al 1990).

In fact, clinical evidence has shown that paroxysms of atrial fibrillation occur more frequently and for significantly longer in patients receiving digoxin (Galun et al 1991, Rawles et al 1990). Furthermore, during an episode of paroxysmal atrial fibrillation, the initial heart rate appears no better controlled whether or not digoxin is being taken (Galun et al 1991, Rawles et al 1990). The mechanism for this is unclear but digoxin increases vagal tone, moderating the speed of atrioventricular conduction, and also reduces the atrial refractory period. This latter property may paradoxically render the atrium more susceptible to fibrillation and may reduce or even prevent the chance of reversion to sinus rhythm (Rawles et al 1990).

Verapamil

Another commonly prescribed drug, verapamil increases atrioventricular block and the effective refractory period of the atrioventricular node. This results in control of the 
ventricular rate in sustained atrial fibrillation and may lead to significantly improved exercise capacity (Klein and Kaplinsky 1982).

Whilst occasionally effective in converting atrial fibrillation to sinus rhythm, verapamil has, however, a much lower rate of conversion than either amiodarone, flecainide, propafenone or esmolol (Kondili et al 1990, Noc et al 1990, Platia et al 1989, Suttorp et al 1989). Although this is obviously indirect evidence, these studies nevertheless suggest that verapamil may be relatively ineffective in controlling paroxysmal atrial fibrillation. In addition, its administration to patients with underlying Wolf-Parkinson-White syndrome who present with paroxysmal atrial fibrillation may occasionally lead to serious adverse effects, including ventricular fibrillation and severe haemodynamic impairment (Strasberg et al 1989).

\section{Class I antiarrhythmics}

Class I antiarrhythmics remain popular drugs for use in paroxysmal atrial fibrillation. It is important, however, to distinguish between paroxysmal atrial fibrillation and flutter, as the use of a Class 1 agent (for example, quinidine, disopyramide and flecainide) in patients with atrial flutter may accelerate the ventricular response with a 1:1 atrioventricular conduction (Nathan et al 1984, Singh et al 1991). This may be due in part to the slowing in atrial rate and the anticholinergic effects (especially with disopyramide and quinidine) which result in an increase in atrioventricular conduction (Nathan et al 1984, Singh et al 1991). With atrial flutter it is therefore advisable to coadminister these agents with digoxin, beta-blocker or verapamil to avoid the potential for the accelerated atrioventricular conduction (Nathan et al 1984, Singh et al 1991).

Quinidine remains a frequently used Class la drug especially in North America for maintaining sinus rhythm after cardioversion or for reducing the number of paroxysms of atrial fibrillation (Pritchett 1992). This stratagem is supported by a meta-analysis of six controlled trials which showed that patients treated with quinidine were less likely to have a recurrence of atrial fibrillation (Coplen et al 1990). Importantly however this analysis also demonstrated an excess of mortality for the treated group - total mortality in the quinidine group (12 deaths) was significantly 
higher than in the control group (3 deaths) (Coplen et al 1990). Quinidine therapy also interacts with concurrent digoxin administration and is associated with serious side effects such as precipitation of polymorphic ventricular tachycardia, blood dyscrasias and cinchonism (Singh et al 1991).

Another Class 1a drug, disopyramide, may also prevent recurrences of paroxysmal atrial fibrillation but its effects are inconsistent (Ito et al 1989). It is also poorly tolerated, particularly in the elderly and those with glaucoma and prostatism, due primarily to its profound anticholinergic properties (Singh et al 1991). Procainamide also shares some electrophysiological properties with quinidine and disopyramide, but to date there have been no placebo-controlled studies of this drug (Pritchett 1992).

In contrast, Class Ic antiarrhythmics such as flecainide have been well investigated. They have very potent effects on conduction within cardiac cell membranes and lengthen the PR interval and QRS complex on the electrocardiogram. Flecainide, for example, has been shown to be effective in preventing recurrences of paroxysmal atrial fibrillation in up to $60 \%$ of patients but the drug does not limit the ventricular response (Sonnhag et al 1988). Studies of flecainide have also confirmed a significant increase in the median length of time to first recurrence of atrial fibrillation (Anderson et al 1989, Pietersen and Hellemann 1991). Adverse effects have been reported in up to $74 \%$ of patients, but these were mostly tolerable (Anderson et al 1989, Pietersen and Hellemann 1991). Nevertheless, doubts on the safety of flecainide have been raised by the Cardiac Arrhythmia Suppression Trial, in which patients (post myocardial infarction) given flecainide for ventricular arrhythmias had a worsened prognosis (Echt et al 1991). The risks to patients with atrial fibrillation and other supraventricular arrhythmias, however, are not known (Pratt et al 1990).

Propafenone, another class Ic compound, may also be effective in paroxysmal atrial fibrillation (Connolly and Hoffert 1989, Pritchett et al 1991). This is particularly pertinent in view of its inherent rate limiting (class II) properties, allowing potentially greater ventricular rate control. In one study, however, of 50 patients with paroxysmal atrial fibrillation, flecainide was 
found to be more effective than propafenone in converting patients to sinus rhythm (with a $90 \%$ vs $55 \%$ conversion rate to sinus rhythm respectively) (Suttorp et al 1990). Propafenone has also been compared to sotalol and both are equally effective in maintaining sinus rhythm in patients with recurrent atrial fibrillation (Reimold et al 1993).

\section{Class III antiarrhythmics}

Sotalol, another commonly prescribed drug, combines both Class II (beta blockade) and Class III (prolongation of repolarisation) antiarrhythmic effects. This latter property is thought mainly due to the dextro (d) isomer of the sotalol compound (Kato et al 1986, Lynch et al 1985). There is little evidence, however, substantiating the efficacy of sotalol specifically in paroxysmal atrial fibrillation. Both the conventional racemic mixture and d-sotalol have been found effective in generally treating paroxysmal supraventricular tachycardias (Sahar et al 1989). Sotalol also appears effective in controlling the ventricular response in atrial fibrillation (Juul-Möller et al 1990).

Another Class III drug, amiodarone, is a very effective drug for the treatment of paroxysmal atrial fibrillation but its use has to be moderated by its potentially serious, albeit relatively rare, side effects (Singh et al 1991). These are, however, more common with higher doses of amiodarone and with prolonged therapy, although some side effects may be reversible on withdrawal of the drug (Singh et al 1991). Nevertheless, amiodarone is of particular value in paroxysmal atrial fibrillation for treating patients refractory to other measures, and low doses (often much less than those required for the control of ventricular arrhythmias) may be effective with little risk of side effects. For example, amiodarone has been shown to be highly effective in converting paroxysmal atrial fibrillation to sinus rhythm (Noc et al 1990) and has been shown to be capable of maintaining sinus rhythm long-term (Blevins et al 1987). Amiodarone also appears more effective than either verapamil (Noc et al 1990) or quinidine (Vitolo et al 1981) and is highly effective in controlling symptoms possibly by moderating the ventricular response even if an attack occurs (Horowitz et al 1985). The drug can be used both orally and intravenously, and unlike oral 
administration (which has a slow onset of action due to the long half-life of the drug), intravenous amiodarone may act relatively rapidly (Singh et al 1991). However, there are seldom indications for the use of a rapid loading regimen using intravenous amiodarone in the suppression of paroxysmal atrial fibrillation. Care should also be taken when using amiodarone as it can significantly increase the plasma concentrations of digoxin thereby leading to potential toxicity (Singh et al 1991). It can also lead to overanticoagulation in patients taking warfarin, perhaps by a hepatic interaction (Hamer et al 1982).

\section{Non-drug therapy}

If paroxysmal atrial fibrillation is part of the spectrum of the sick sinus syndrome then permanent pacemaker implantation should be considered. Either an atrial or dual chamber pacemaker should be used, dependent upon the integrity of the atrio-ventricular pathway. This is further discussed below. If successful control of the arrhythmia cannot be achieved then, as a last resort, ablation of the atrioventricular node (with concomitant implantation of a rate-responsive permanent pacemaker) could be contemplated (Egorov et al 1990, Zipes 1992a).

Surgical therapy for paroxysmal atrial fibrillation has also been advocated but although effective, it is unlikely (at least in the United Kingdom) to become a generally useful therapeutic option except for specific groups (Cox et al 1991, Defauw et al 1992, Leitch et al 1991). The two most promising surgical options are the 'corridor' and 'maze' procedures. The 'corridor' procedure effectively isolates both left and right atria, leaving a strip of myocardium connecting the sinus node to the atrioventricular node (Defauw et al 1992). This procedure does not prevent atrial fibrillation, but essentially 'isolates' the fibrillating atria from a strip of tissue connecting the sinus and atrioventricular nodes. In contrast, the 'maze' procedure attempts to completely prevent atrial fibrillation by channelling the atrial activation between a series of incisions (Cox et al 1991). This latter procedure therefore restores coordinated atrial as well as ventricular electrical activity, allowing atrial transport with good haemodynamic results and low 
thromboembolic risk. Initial reports from these procedures are encouraging, but post-operative pacemaker implantation may still be necessary (Defauw et al 1992).

\section{Sick sinus syndrome}

One condition commonly associated with paroxysmal atrial fibrillation is sick sinus syndrome. Management of this condition deserves special discussion. It is usually a disease of the elderly, which is produced by idiopathic degeneration of the sinoatrial node, and its manifestations can range from asymptomatic to nonspecific. These include dizziness, palpitations, fatigue, syncope and even sudden death.

Electrocardiographic evidence of sick sinus syndrome includes inappropriate sinus bradycardia, sinus pauses or arrest, and sinus exit block. These bradycardias may alternate with tachyarrhythmias, especially slow and fast atrial fibrillation, resulting in the 'tachy-brady' syndrome.

The use of drugs such as digoxin, beta-blockers and calcium antagonists may initiate or exacerbate symptoms in these patients. In the presence of symptomatic bradyarrhythmias, permanent pacing is indicated. There is evidence that simple ventricular pacing systems may result in an increase in the incidence of permanent and paroxysmal atrial fibrillation, congestive heart failure and worsened prognosis (Hesselson et al 1992, Rosenqvist et al 1988, Stangl et al 1990, Zanini et al 1990). Regular atrial pacing, in contrast, either alone or combined with ventricular pacing, is often effective in preventing paroxysmal atrial fibrillation associated with the tachy-brady syndrome (perhaps by stabilising the atrium electrically) and can therefore improve the overall prognosis (Ohe et al 1987, Stangl et al 1990).

Patients with sick sinus syndrome also appear to be at increased thromboembolic risk (Bathen et al 1978, Fairfax et al 1976, Rosenqvist et al 1988), and this is thought to be associated with the tachy-brady syndrome. Therefore, formal anticoagulation should also be initiated in addition to antiarrhythmic therapy. 
Prophylaxis against thromboembolic events

Some physicians seem to regard paroxysmal atrial fibrillation as conferring a greater risk of serious thrombo-embolic complications than chronic atrial fibrillation. The data available however does not substantiate such beliefs and patients appear to be in an intermediate risk category between normal sinus rhythm and chronic atrial fibrillation.

Data from the Framingham study has shown that men with chronic atrial fibrillation had an annual stroke rate of $5.4 \%$ (with a relative risk of 4.7 ), whereas for paroxysmal atrial fibrillation the corresponding figure was 1.3\% (and 0) (Kannel et al 1983). Other studies have also confirmed similar or lower incidences of thrombo-emboli for paroxysmal as opposed to chronic atrial fibrillation (Petersen and Godtfredsen 1986, Petersen et al 1989, Shimomura et al 1989, Takahashi et al 1981). For example, in a Danish study of 426 patients, the mean yearly incidence of systemic emboli was $2.0 \%$ for paroxysmal atrial fibrillation, which rose to $5.6 \%$ after progression to chronic atrial fibrillation (Petersen and Godttredsen 1986). Further evidence for a low thromboembolic risk in these patients comes from a computed tomography (CT Scan) study where no significant differences in cerebral infarction were seen in patients with paroxysmal atrial fibrillation when compared to matched controls in sinus rhythm (Petersen et al 1989).

In contrast, however, the Montreal Heart Institute study (although retrospective) demonstrated that the incidence of embolic events associated with paroxysmal atrial fibrillation was similar to that observed with chronic atrial fibrillation (Roy et al 1986). Paroxysmal, intermittent and recent-onset atrial fibrillation seem particularly hazardous, with an excess of strokes, especially during the transition to chronic atrial fibrillation (Petersen 1990a, Petersen 1990b, Selzer 1982). In addition, the presence of spontaneous echo contrast during paroxysms of atrial fibrillation has been described in a patient who presented with a cerebral embolus (Obarski et al 1990). As spontaneous echo contrast is associated with systemic emboli in sustained atrial fibrillation, its presence during paroxysms of paroxysmal atrial fibrillation is consistent with evidence for some thromboembolic risk in these patients. 
An important risk factor however is the influence of underlying pathology, such as rheumatic heart disease and hypertension. For example, there is evidence demonstrating that the presence of rheumatic heart disease is a major additive risk factor with respect to progression to both thrombo-embolic events and chronic atrial fibrillation (Takahashi et al 1981). In addition, a study of 63 consecutive patients with paroxysmal atrial fibrillation demonstrated that an older age, hypertension and an increased left atrial diameter (on 2-D echocardiography) were risk factors for systemic embolism (Corbalan et al 1992). Another important group at risk are those patients with paroxysmal atrial tachyarrhythmias associated with the sick sinus syndrome (Bathen et al 1978, Fairfax et al 1976, Rosenqvist et al 1988).

Although there have been five major studies establishing the benefits of antithrombotic therapy in chronic atrial fibrillation, there are few data available for paroxysmal atrial fibrillation (Boston Area Anticoagulation Trial for Atrial Fibrillation Investigators 1990, Connolly et al 1991, Ezekowitz et al 1992, Petersen et al 1989, Stroke Prevention in Atrial Fibrillation Investigators 1991). This is unfortunate as with the overall risk of thrombo-embolic events being lower, the benefits of warfarin therapy may be significantly offset by its associated morbidity. Aspirin therapy may be safer and appears to be reasonably effective in chronic atrial fibrillation, at least for those patients aged below 75 years with no structural heart disease (Stroke Prevention in Atrial Fibrillation Investigators 1990). In similarity to warfarin, however, its effects in paroxysmal atrial fibrillation are unknown. The precise role of antithrombotic therapy thus remains to be defined.

\section{Discussion}

Following appropriate investigation, patients with symptomatic paroxysms of atrial fibrillation should be treated using both an antiarrhythmic and an antithrombotic agent. Although accepting the paucity of supporting evidence, treatment should be initiated using sotalol, reserving Class 1 agents for second choice. If patients do not have underlying coronary artery disease, Class 1c agents (for example, propafenone, in view of its rate limiting properties, or 
flecainide) could be used as second line therapy. Class 1a agents (for example, quinidine), amiodarone and ablation of the atrio-ventricular pathway are further useful options for the problematic case. Despite its potential toxicity, amiodarone can be of particular value in the elderly. This group are often intolerant of alternative therapy and it is often possible to achieve good control with very low doses of amiodarone (i.e. $100 \mathrm{mg}$ daily or lower). At this dosage the risks of serious toxicity should be low.

There is little evidence however to support the traditional use of digoxin or verapamil. In patients with sick sinus syndrome, atrial or dual chamber pacing should be considered in addition to antithrombotic therapy. Intractable disabling paroxysmal atrial fibrillation should, however, be referred for specialist advice. The role of antithrombotic therapy is unclear but until clarification becomes available it is advisable to give all patients an antithrombotic agent. Those patients deemed to be at high risk, for example, patients with underlying structural abnormalities (such as rheumatically damaged valves, patients who have already experienced a prior thromboembolic event or poor left ventricular contraction) or sick sinus syndrome should be treated by formal anticoagulation. Patients with frequent paroxysms of atrial fibrillation are most probably at increased risk and should be formally anticoagulated. It would seem reasonable to treat all other patients with aspirin. 


\subsection{STUDIES OF PLASMA FIBRINOGEN AND D-DIMER IN PAROXYSMAL ATRIAL FIBRILLATION}

Introduction

Paroxysmal atrial fibrillation may confer a significant risk of serious thromboembolic complications, although whether this risk is any greater than patients in chronic atrial fibrillation remains controversial. As discussed above, however, the data available (especially from epidemiological studies) seems to put patients with paroxysmal atrial fibrillation in an intermediate risk category between normal sinus rhythm and chronic atrial fibrillation (Petersen 1990).

As discussed in chapter 3, patients with chronic atrial fibrillation have significant abnormalities of plasma fibrinogen and fibrin degradation products (fibrin D-dimer). If patients with paroxysmal atrial fibrillation are at intermediate thrombo-embolic risk, and if plasma fibrinogen and fibrin D-dimer were suitable indicators of thrombogenesis, I would hypothesise that intermediate levels of these plasma markers will be found in patients with paroxysmal atrial fibrillation when compared to patients with chronic atrial fibrillation and sinus rhythm. The aim of the study reported in this chapter was to determine whether or not paroxysmal atrial fibrillation is associated with increased plasma fibrinogen or D-dimer levels; and if so, whether or not such levels were related to any underlying cardiac abnormalities.

\section{Materials and Methods}

A prospective case controlled study of male and female patients with paroxysmal atrial fibrillation was undertaken. Paroxysmal atrial fibrillation was defined as episodes of atrial fibrillation lasting for 10 or more complexes documented on at least two occasions by a 24 hour Holter monitor put on outpatients, or the presence of similar paroxysms noted on a cardiac monitor during an inpatient stay. Exclusion criteria were similar to that described in chapter 3. 
Standard two dimensional and Doppler echocardiography was also performed in all patients using a Hewlett-Packard Sonos 500 machine (Hewlett-Packard, Arondale, Pa, USA.) equipped with a standard $2.5 \mathrm{MHz}$ transducer, using techniques and methods previously described in chapter 3 . Using the standard video facility of the ultrasound machine, off line analytical methodology was again used to quantify measurements. Assays for plasma fibrinogen and fibrin D-dimer levels were as previously described (chapter 3).

Plasma fibrinogen and fibrin D-dimer results for patients were compared to the 85 patients in chronic atrial fibrillation described in chapter 3 (56 of whom were not on warfarin therapy), 27 hospital controls lage \pm 5 years and sex-matched patients with coronary artery disease and with normal left ventricular function] and 158 population controls (patients aged 5565 years, sampled from a local population study, the North Glasgow MONICA Study). Echocardiographic data for the patients with paroxysmal atrial fibrillation were compared to corresponding results for patients with chronic atrial fibrillation (as described in chapter 3) on no antithrombotic therapy and 14 healthy volunteers which served as 'normal' echocardiograms.

All the values were expressed as median \pm interquartile range (IQR). Statistical comparisons were performed using the Kruskal-Wallis test and Mann-Whitney two sample rank test for non-parametric distributions; and the 95\% confidence intervals (c.i.) calculated for the point estimate of the difference in medians. Correlations between clinical variables or rheological measurements were performed using Spearman's Rank correlation method. A probability of $\leq 0.05$ was considered statistically significant.

Results

A total of 33 patients ( 18 male and 15 female; mean age 60.8 years, s.e.m.1.42) with paroxysmal atrial fibrillation were studied. At the time of the study, 3 patients were taking warfarin therapy and their plasma D-dimer results were excluded from further analyses (see chapter 3). A past history of ischaemic heart disease was present in 15, mitral valve disease in 2 
and Wolf-Parkinson-White Syndrome in one patient. There were six smokers in the paroxysmal atrial fibrillation group.

For the whole patient group, patients with paroxysmal atrial fibrillation had intermediate levels of plasma fibrinogen (median 3.07g/; IQR 2.79-3.76) when compared to patients with chronic atrial fibrillation (median 3.58g/l; IQR 3.0-4.7) and sinus rhythm (hospital controls - median 2.85g/l; IQR 2.48-3.12; population controls - median $2.6 \mathrm{~g} / \mathrm{l}$; IQR 2.23-3.0). These differences were statistically significant (Kruskal-Wallis test $H=86.6$, d.f. $=3 ; p<0.001$ ) (Table 5.1).

As warfarin (but not aspirin) significantly influences intravascular clotting and therefore plasma D-dimer levels, patients with atrial fibrillation who were not on warfarin therapy were compared further (Table 5.2). Patients with chronic atrial fibrillation who were not on warfarin therapy ( $n=56)$ again had the highest median plasma fibrinogen (3.79g/l; IQR 3.0-4.7) and fibrin Ddimer (146ng/ml; IQR 78-228) levels. Patients with paroxysmal atrial fibrillation $(n=30)$ however had intermediate levels of median plasma fibrinogen (3.04g/l IQR 2.79-3.75) and plasma fibrin Ddimer (97ng/ml IQR 53-140) when compared to patients with chronic atrial fibrillation and controls in sinus rhythm. These differences were again statistically significant (for plasma fibrinogen: Kruskal-Wallis test $H=70.4$, d.f. $=3 ; p<0.001$; and for D-dimer: Kruskal-Wallis test $H=58.8$, d.f. $=3$; $p<0.001$ ) (Table 5.2).

Suitable left and right atrial echocardiographic measurements were obtained in 23 and 17 patients with paroxysmal atrial fibrillation (who were not on warfarin) respectively. However, technically adequate M-Mode left ventricular measurements were obtained in only 13 patients. They were compared to atrial echocardiographic measurements of 56 patients with chronic atrial fibrillation who were not on warfarin therapy (obtained from chapter 3 ) and 14 healthy patients who served as 'normals' (Table 5.3). Patients with paroxysmal atrial fibrillation had intermediate median left atrial dimensions (anteroposterior, mediolateral and superoinferior), and left and right atrial volumes (ellipsoid) when compared to patients with chronic atrial fibrillation and 'normals' in sinus 
rhythm $(p<0.05)$. There were however no significant differences in left ventricular dimensions and fractional shortening between patients with chronic and paroxysmal atrial fibrillation.

There was a weak correlation between age and plasma fibrinogen in patients with paroxysmal atrial fibrillation (Table 5.4). There were no significant correlations between body mass index, left atrial dimension, left atrial volume, right atrial volume, left ventricular dimensions or fractional shortening with plasma fibrinogen or fibrin D-dimer levels (Table 5.4).

\section{Discussion}

This study demonstrates intermediate levels of plasma fibrinogen and fibrin Ddimer levels in patients with paroxysmal atrial fibrillation when compared to similar patients in chronic atrial fibrillation and those in sinus rhythm. The intermediate levels of plasma fibrinogen gives added support to my hypothesis that haemorheological factors may partly account for the risk of thrombogenesis in atrial fibrillation, which also appears intermediate in paroxysmal atrial fibrillation (when compared to chronic atrial fibrillation and sinus ihythm). The elevated levels of plasma fibrinogen in these patients are not just due to underlying coronary artery disease, as plasma levels in patients with paroxysmal atrial fibrillation were significantly higher when compared to levels in age- and sex-matched hospital controls who were patients with known ischaemic heart disease attending the outpatient clinic.

As demonstrated in chapter 3 , patients with chronic atrial fibrillation are at high thromboembolic risk and the highest levels of plasma fibrin D-dimer in these patients indicate greatly enhanced fibrin turnover and intravascular clotting in these patients. Epidemiological data from the Framingham Study demonstrate that patients with paroxysmal atrial fibrillation are at lower thromboembolic risk when compared to patients with chronic atrial fibrillation. This study provides rheological support for this, as intermediate plasma levels of fibrinogen and fibrin D-dimer are found in these patients. Although patients with chronic atrial fibrillation had significantly higher left atrial dimensions and left atrial volumes when compared to patients with paroxysmal atrial fibrillation, there was no significant correlation between plasma fibrinogen or fibrin D-dimer with 
atrial dimension or volume, as demonstrated in chapter 3. There was also no difference in left ventricular function (as assessed by fractional shortening) between patients with chronic and paroxysmal atrial fibrillation. Finally, there were no significant correlations between left atrial dimension, left atrial volume, left ventricular fractional shortening or body mass index with plasma fibrinogen or D-dimer in patients with paroxysmal atrial fibrillation. These findings suggest that it is atrial fibrillation itself which is the main contributory factor to thromboembolic risk, with little contribution from structural factors, such as left atrial size.

As discussed in chapter 3 the phenomenon of spontaneous echo contrast may provide visual demonstration of blood stasis within the left atrial cavity. This may predispose to thrombus formation and is unrelated to age, gender, left ventricular dysfunction or anticoagulant therapy (Black et al 1991, Castello et al 1990, Manning 1993). However, spontaneous echo contrast is not a universal finding in all patients with chronic atrial fibrillation, possibly accounting for the variability in plasma fibrinogen and fibrin D-dimer in these patients. In paroxysmal atrial fibrillation, however, transient spontaneous echo contrast has been demonstrated within the left atrium during a paroxysm of atrial fibrillation suggesting that intermittent flow abnormalities or stasis may exist in these patients (Obarski et al 1990). On theoretical grounds, patients with the most frequent paroxysms of atrial fibrillation would be at highest risk of flow abnormalities or stasis and these patients would therefore be at greatest thromboembolic risk. Unfortunately, my study was not designed to quantify the episodes of atrial fibrillation and to correlate these frequency of paroxysms with plasma fibrinogen or fibrin D-dimer levels.

This study provides therefore haemorheological support for the increased risk of thromboembolism in all patients with atrial fibrillation. In addition, patients with paroxysmal atrial fibrillation have intermediate levels of plasma fibrinogen and fibrin D-dimer when compared to patients in chronic atrial fibrillation and sinus rhythm. This is in keeping with the majority of the literature and suggests that whilst the group of patients with paroxysmal atrial fibrillation are at an intermediate risk of stroke and thromboembolism, measurement of fibrinogen and fibrin D-dimer 
may allow individual risk stratification. This study also supports the hypothesis that plasma D-dimer may provide a suitable marker for intravascular thrombogenesis and thromboembolic risk. 
TABLE 5.1

PAROXYSMAL ATRIAL FIBRILLATION AND

PLASMA FIBRINOGEN LEVELS

(WHOLE STUDY GROUP)

WHOLE GROUP

number

(n)

$M: F$ age

mean

(s.e.m.) plasma fibrinogen

median

g/l (IQR)

\section{paroxysmal atrial}

fibrillation

chronic atrial fibrillation

33

$18 \mathrm{M} ; 15 \mathrm{~F}$

85

43M; $42 \mathrm{~F}$

27

$14 \mathrm{M} ; 13 \mathrm{~F}$

158

$88 \mathrm{M} ; 70 \mathrm{~F}$

HOSPITAL

CONTROLS

POPULATION

CONTROLS

61

(1.42)

$3.07^{\star}$

(2.79-3.76)

(1.42) (2.79-3.76)




\section{TABLE 5.2}

PLASMA FIBRINOGEN AND D-DIMER IN

PAROXYSMAL ATRIAL FIBRILLATION

(NO WARFARIN GROUPS)

NO WARFARIN

THERAPY number

(n) plasma fibrinogen

median

g/l(s.e.m.) plasma fibrin

D-dimer

median

ng/ml(s.e.m.)

\section{paroxysmal atrial}

fibrillation
30

$3.04^{*}$

(2.79-3.75)
97@

(53-140)

\begin{tabular}{lccc}
\hline chronic atrial fibrillation & 56 & 3.79 & 146 \\
& & $(3.0-4.7)$ & $(78-228)$
\end{tabular}

\section{HOSPITAL}

27

2.85

38

CONTROLS

(2.48-3.12)

(30-59)

\section{POPULATION}

CONTROLS

158

2.60

(2.23-3.0)

(54-104)

\section{PLASMA FIBRINOGEN}

* significantly different when compared to chronic atrial fibrillation group (Mann-Whitney test, $p=0.011)$, hospital controls $(p=0.044)$ and population controls $(p<0.0001)$.

\section{PLASMA FIBRIN D-DIMER}

@ significantly different when compared to chronic atrial fibrillation group (Mann-Whitney test, $p=0.012)$, hospital controls $(p<0.0001)$ and population controls $(p=0.045)$. 


\section{TABLE 5.3 \\ ECHOCARDIOGRAPHY AND \\ PAROXYSMAL ATRIAL FIBRILLATION}

(no warfarin groups)

\author{
paroxysmal \\ atrial fibrillation
}

patients with

echocardiograms

\section{LEFT ATRIUM}

D 1

cm (IQR)

D 2

cm (IQR)

D 3

cm (IQR)

\section{LEFT ATRIAL}

VOLUME

$\mathrm{CM}^{3}$ (IQR)

RIGHT ATRIAL

VOLUME

$\mathrm{CM}^{3}$ (IQR)

\section{LEFT}

\section{VENTRICLE}

end-diastolic

dimension

cm (IQR) $4.15^{\star}$

(3.56-4.47)

4.03*

(3.80-4.30)

$5.12^{\star}$

(4.62-5.5)

32.8

(25.3-41.4)

27.9*

$6.02^{*}$

(5.32-6.59)
(21.0-30.6) chronic atrial

fibrillation

54

14

normals

3.37

(3.09-3.65)

3.67

(3.8-4.3)

(4.20-4.79)

4.61

(4.2-4.79)

(5.08-6.90)

$48.8^{\star *}$

29.2

(38.7-65.2)

(26.3-31.5)

42.4

20.1

(35.3-56.7)

(17.2-23.0)
5.5

(5.0-6.1)
5.17

(4.86-5.50) 
end-systolic

dimension

cm (IQR)

fractional

shortening

$\%$ (IQR)
4.29*

(3.76-5.2)

4.14

3.4

(3.24-4.88)

(3.24-3.60)

28.16*

24.0

35.5

(21.0-30.6)

$\%(I Q R)$

* significantly different when compared to healthy controls $(p \leq 0.01)$;

* * significantly different when compared to patients with paroxysmal atrial fibrillation $(p \leq 0.01)$. 
TABLE 5.4

PAROXYSMAL ATRIAL FIBRILLATION

- SPEARMAN CORRELATIONS

FIBRINOGEN D- DIMER

\begin{tabular}{|c|c|c|c|c|}
\hline & $\mathbf{r}$ & $p$ & $r$ & $\mathbf{p}$ \\
\hline age & 0.8 & $0.046^{*}$ & 0.30 & 0.12 \\
\hline $\begin{array}{l}\text { body mass } \\
\text { index }\end{array}$ & -0.04 & 0.83 & -0.23 & 0.26 \\
\hline $\begin{array}{l}\text { Left atrial } \\
\text { dimension } \\
\text { (d1) }\end{array}$ & 0.096 & 0.689 & 0.16 & 0.51 \\
\hline $\begin{array}{l}\text { Left atrial } \\
\text { volume }\end{array}$ & 0.39 & 0.09 & 0.16 & 0.51 \\
\hline $\begin{array}{l}\text { Right atrial } \\
\text { volume }\end{array}$ & 0.25 & 0.63 & 0.25 & 0.64 \\
\hline $\begin{array}{l}\text { Left ventricle } \\
\text { end-diastolic } \\
\text { dimension }\end{array}$ & 0.55 & 0.074 & -0.04 & 0.91 \\
\hline $\begin{array}{l}\text { Left ventricle } \\
\text { end-systolic } \\
\text { dimension }\end{array}$ & 0.56 & 0.069 & -0.03 & 0.93 \\
\hline $\begin{array}{l}\text { Left } \\
\text { ventricular } \\
\text { fractional } \\
\text { shortening }\end{array}$ & -0.48 & 0.13 & -0.14 & 0.69 \\
\hline
\end{tabular}

${ }^{*} p<0.05$ 


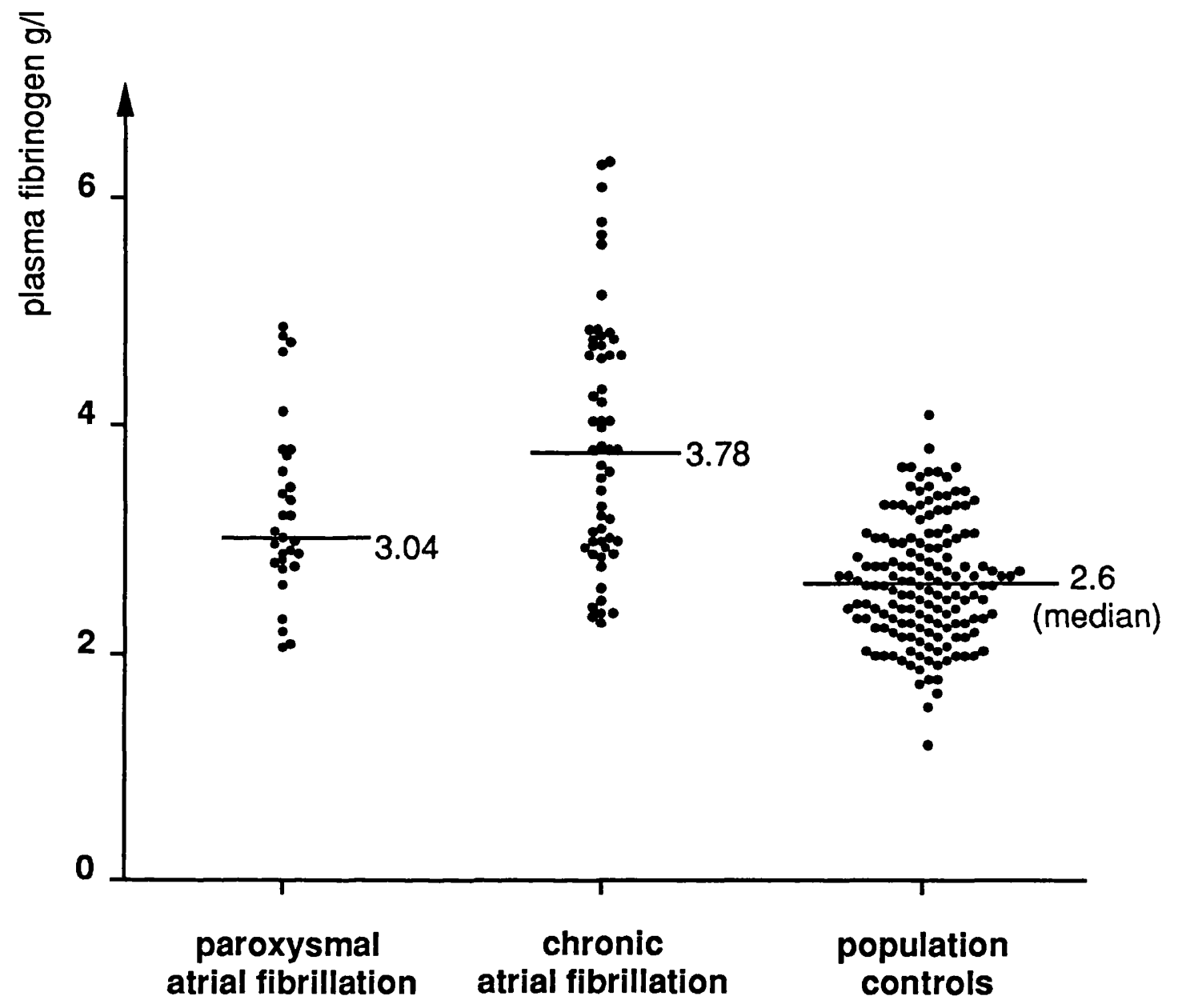

FIGURE 5.1

PLASMA FIBRINOGEN AND PAROXYSMAL ATRIAL FIBRILLATION 


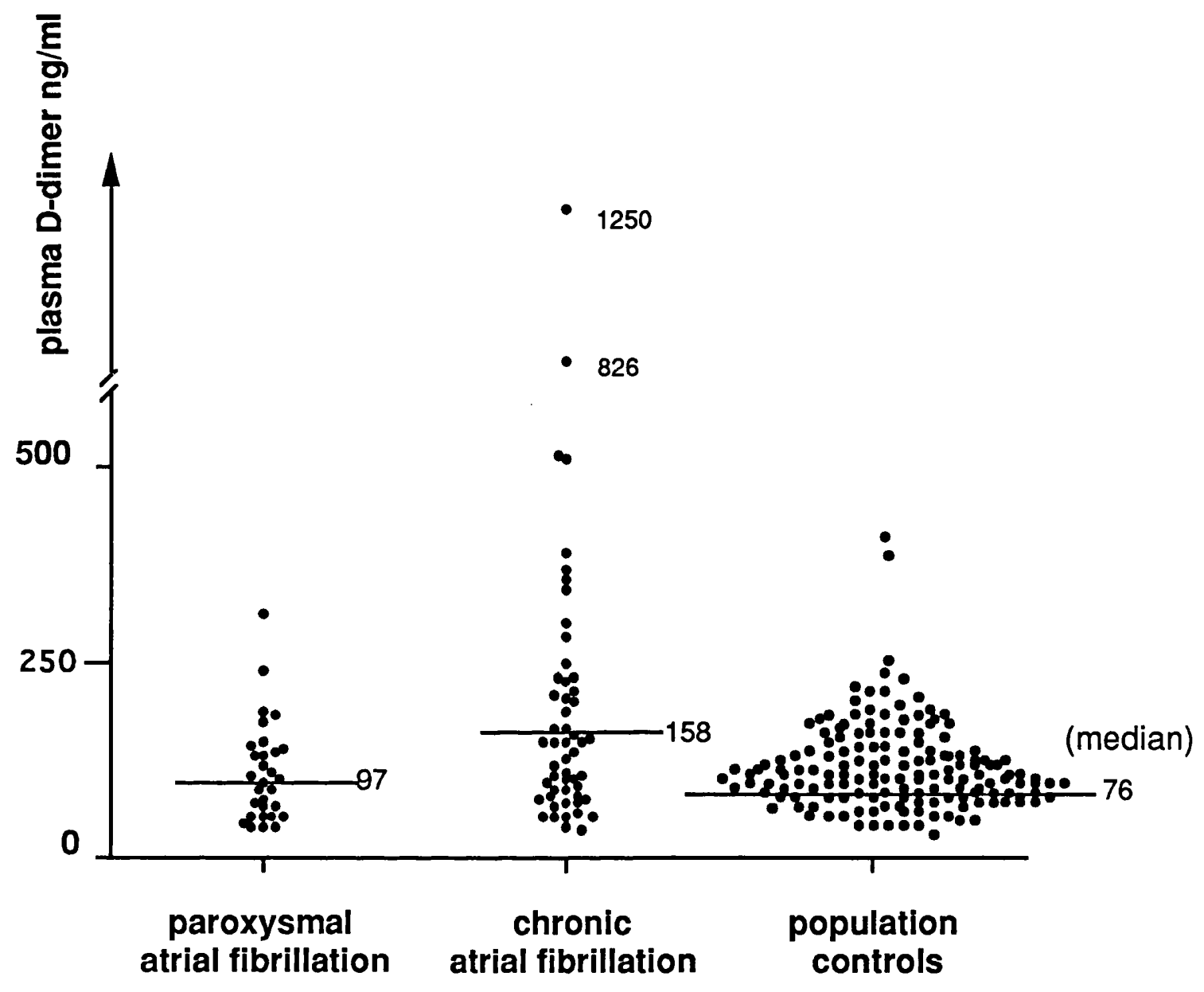

FIGURE 5.2

PLASMA D-DIMER AND PAROXYSMAL

ATRIAL FIBRILLATION 
CHAPTER 6

THROMBOGENESIS

IN

PAROXYSMAL

SUPRAVENTRICULAR

TACHYCARDIA 


\subsection{STUdies OF PLASMA FIBRINOGEN AND D-DIMER IN PAROXYSMAL SUPRAVENTRICULAR TACHYCARDIA}

\section{Introduction}

Contrary to popular belief, supraventricular tachycardia is not an entirely benign condition. Patients can be considerably symptomatic and haemodynamic sequelae (for example, heart failure), can often occur. There is some evidence, for example, that chronic supraventricular tachycardia can result in left ventricular dysfunction, left ventricular dilatation and occasionally even a dilated cardiomyopathy (Packer et al 1986, Spinale et al 1991, Tomita et al 1991, Zipes 1992b). Such left ventricular dilatation and functional impairment may constitute an important thromboembolic risk, as reviewed and explored in chapter 8. Interestingly, termination of the arrhythmia improves symptoms of heart failure. There is also experimental evidence that supraventricular tachycardia (especially chronic) may result in systolic and diastolic dysfunction, with reduced collagen support of adjoining myocytes (Spinale et al 1991, Tomita et al 1991). In these studies, early recovery from the arrhythmia was associated with left ventricular hypertrophy, increased collagen synthesis and increased left ventricular stiffness; all leading to diastolic dysfunction (Spinale et al 1991, Tomita et al 1991). Despite the important haemodynamic and structural consequences, the risks of thromboembolism in patients with supraventricular tachycardia have not been studied.

There is clinical evidence that patients with paroxysmal atrial fibrillation are at intermediate risk of thromboembolism when compared with patients in chronic atrial fibrillation and subjects in sinus rhythm. In chapter 4 , I have demonstrated that these patients also have intermediate levels of plasma fibrinogen and fibrin D-dimer. My findings are therefore in keeping with clinical observations of a thromboembolic risk which is graded between chronic and paroxysmal atrial fibrillation.

It remains unclear, however, whether it is simply the paroxysmal nature of the arrhythmia which results in the rheological abnormalities; or whether or not it is the incoordinate atrial contractions of atrial fibrillation (whether intermittent or not) which results in the abnormalities in plasma fibrinogen and D-dimer as demonstrated in chapters 3 and 5. Patients with paroxysmal supraventricular tachycardia (and paroxysmal tachycardias other than 
atrial fibrillation) continue to have co-ordinated atrial activity abeit rapid. In view of this, I would hypothesise that it is the atrial fibrillation which is the most important component and if this were true, patients with paroxysmal supraventricular tachycardia (with rapid coordinated atrial activity) should not manifest these haemorheological abnormalities.

The aim of this study therefore was to determine whether the thrombogenic potential (as reflected by abnormalities in plasma fibrinogen or fibrin D-dimer) demonstrated in paroxysmal atrial fibrillation also exists in patients with paroxysmal supraventricular tachycardia.

Methods

A prospective study of patients with paroxysmal tachycardias was undertaken. Patients were identified at outpatient clinics and the nature of the paroxysmal arrhythmia defined by a 24 hour Holter monitor or cardiomemo (transtelephonic monitor). Patients were enrolled if the presence of probable paroxysmal supraventricular tachycardia was documented on the 24hour Holter or cardiomemo. This was defined as the presence of 10 or more complexes of a regular narrow complex tachycardia on one or more occasions. Exclusion criteria were similar to previous studies, namely age greater than 75 years, those with systemic illnesses (liver/renal impairment, malignancy), hypertension, and those who were actively bleeding or requiring transfusion. Patients on warfarin therapy were also excluded.

Blood samples were taken for plasma fibrinogen and fibrin D-dimer and assayed using methods and techniques outlined in chapter 3. Results were compared to 'normal' population values derived from the 2nd MONICA study as in previous chapters.

Statistical comparisons were made using the Mann-Whitney $U$ test for nonparametric populations. A probability of $\leq 0.05$ was considered as representing statistical significance.

Results

A total of 12 patients ( 3 male, 9 female; mean age 51 years, s.e.m. 4.2) were studied. Analysis of the 24hour Holter or cardiomemo demonstrated definite episodes of 
supraventricular tachycardia (PSVT) in nine patients, whilst 3 patients had probable episodes of supraventricular tachycardia. These 12 patients were compared to 12 age ( \pm 5 years) and sex-matched patients (mean age 56 years, s.e.m. 3.1) with paroxysmal atrial fibrillation (PAF), and 'normal' population values derived from 158 subjects in sinus rhythm from a random population survey (2nd North Glasgow MONICA study) as in previous chapters. There were 4 smokers in the PSVT group, whilst the PAF group had 2 smokers.

Patients with PSVT had a lower median plasma fibrinogen level when compared to patients with PAF, but this was of borderline significance only (point estimate difference between medians $0.64 \mathrm{~g} / ;$; $95 \%$ c.i. -0.02 to $1.5, p=0.06$ ). The median plasma fibrinogen level in patients with PSVT (2.6g/l; IQR 2.4-3.0) was also similar to the median level of the population controls (point estimate difference between medians $0.08 \mathrm{~g} /$; $95 \%$ c.i. -0.22 to $0.38, p=0.57, N S$ ).

Patients with PSVT also had a lower median plasma fibrin D-dimer level when compared with patients manifesting PAF (67 vs $95 \mathrm{ng} / \mathrm{mI}$ ), but this did not reach statistical significance (point estimate difference between medians $23 \mathrm{ng} / \mathrm{ml} ; 95 \%$ c.i. -64 to $9, p=0.19$, NS). There was no difference in median plasma fibrin D-dimer level in patients with PSVT when compared with population values (point estimate difference between medians $6 \mathrm{ng} / \mathrm{ml}$; $95 \%$ c.i. -27 to $15, p=0.52, N S)$.

\section{Discussion}

This study demonstrates a trend towards lower plasma fibrinogen and Ddimer levels in patients with paroxysmal supraventricular tachycardia when compared to matched patients with paroxysmal atrial fibrillation. In addition, patients with paroxysmal supraventricular tachycardia had levels of plasma fibrinogen and fibrin D-dimer that were similar to population values derived from a random population sample in sinus rhythm. This was a small study, however, with difficulties in patient recruitment, and only 12 patients with paroxysmal supraventricular tachycardia were studied.

Patients with paroxysmal atrial fibrillation were slightly older than patients in the PSVT group (mean age 51 vs 56 years) and this may have partly accounted for the higher 
plasma fibrinogen levels in the paroxysmal atrial fibrillation group. However, as there were slightly more smokers in the PSVT group, compared to the patients with paroxysmal atrial fibrillation group, it is most unlikely therefore that the elevated plasma fibrinogen or fibrin Ddimer, in patients with paroxysmal atrial fibrillation, was due to an excess of smokers. No comparison of intracardiac dimensions was made in this study as previous chapters have examined this aspect in detail, with no significant effects demonstrable for plasma levels of fibrinogen and fibrin D-dimer.

My findings are consistent with previous observations (chapter 3) that it is likely to be atrial fibrillation itself which is related to the changes in plasma fibrinogen and fibrin D-dimer. This suggests abnormalities in rheology and fibrin turnover in association with this amhythmia. Atrial fibrillation (whether sustained or paroxysmal) is associated with incoordinate, irregular atrial systolic activity and this may result in abnormalities of intracardiac blood flow which may contribute to a prothrombotic state. As discussed in previous chapters, these abnormalities in blood flow are visualised as spontaneous echo contrast during transoesophageal echocardiography, suggesting slow or sluggish blood flow (Black et al 1991). Transient abnormalities of spontaneous echo contrast are also seen during paroxysms of atrial fibrillation (Obarski et al 1990), but to date spontaneous echo contrast has not been documented in patients with paroxysmal supraventricular tachycardia. It is likely that these latter patients have coordinate, regular atrial activity and contractions that would result in a low thromboembolic potential. This is in keeping with the clinical observations of a low thromboembolic risk for these patients with paroxysmal supraventricular tachycardia. My findings are also in keeping with the recommendation that anticoagulation before cardioversion of such patients is not necessary (Dunn et al 1989).

In conclusion, patients with paroxysmal supraventricular tachycardia have a low thromboembolic risk (as reflected by the trend towards lower plasma fibrinogen and fibrin D-dimer levels) when compared to patients with paroxysmal atrial fibrillation. Levels of plasma fibrinogen and fibrin D-dimer in patients with paroxysmal supraventricular tachycardia are in fact similar to population ('normal') values, suggesting that there is no excess thromboembolic 
risk in this group. Further studies with larger numbers of patients with paroxysmal supraventricular tachycardia are required to clearly define this risk, if any. 
TABLE 6.1

\section{PLASMA FIBRINOGEN AND FIBRIN D-DIMER IN}

PAROXYSMAL SUPRAVENTRICULAR TACHYCARDIA

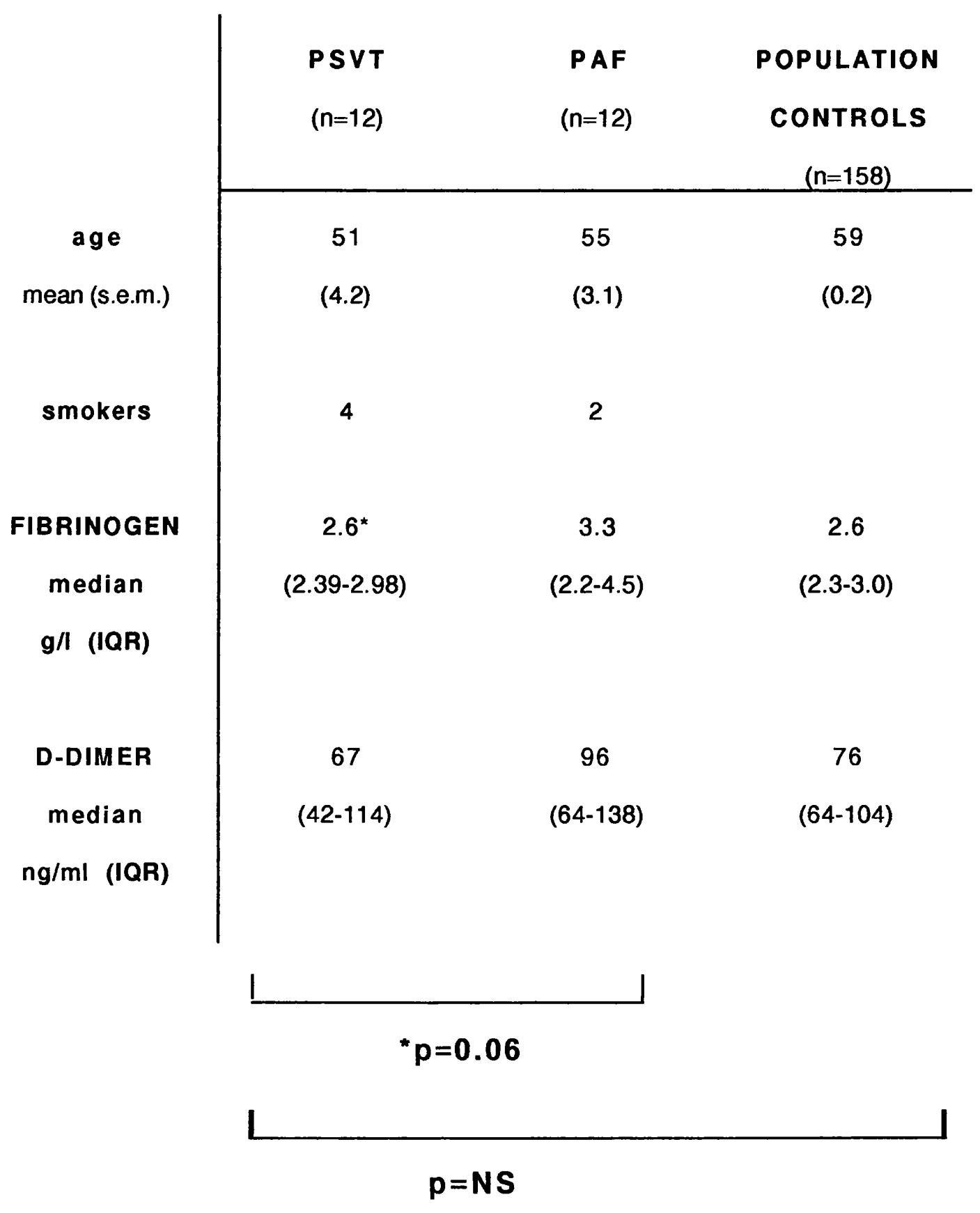

[PSVT=paroxysmal supraventricular tachycardia; $P A F=$ paroxysmal atrial fibrillation] 


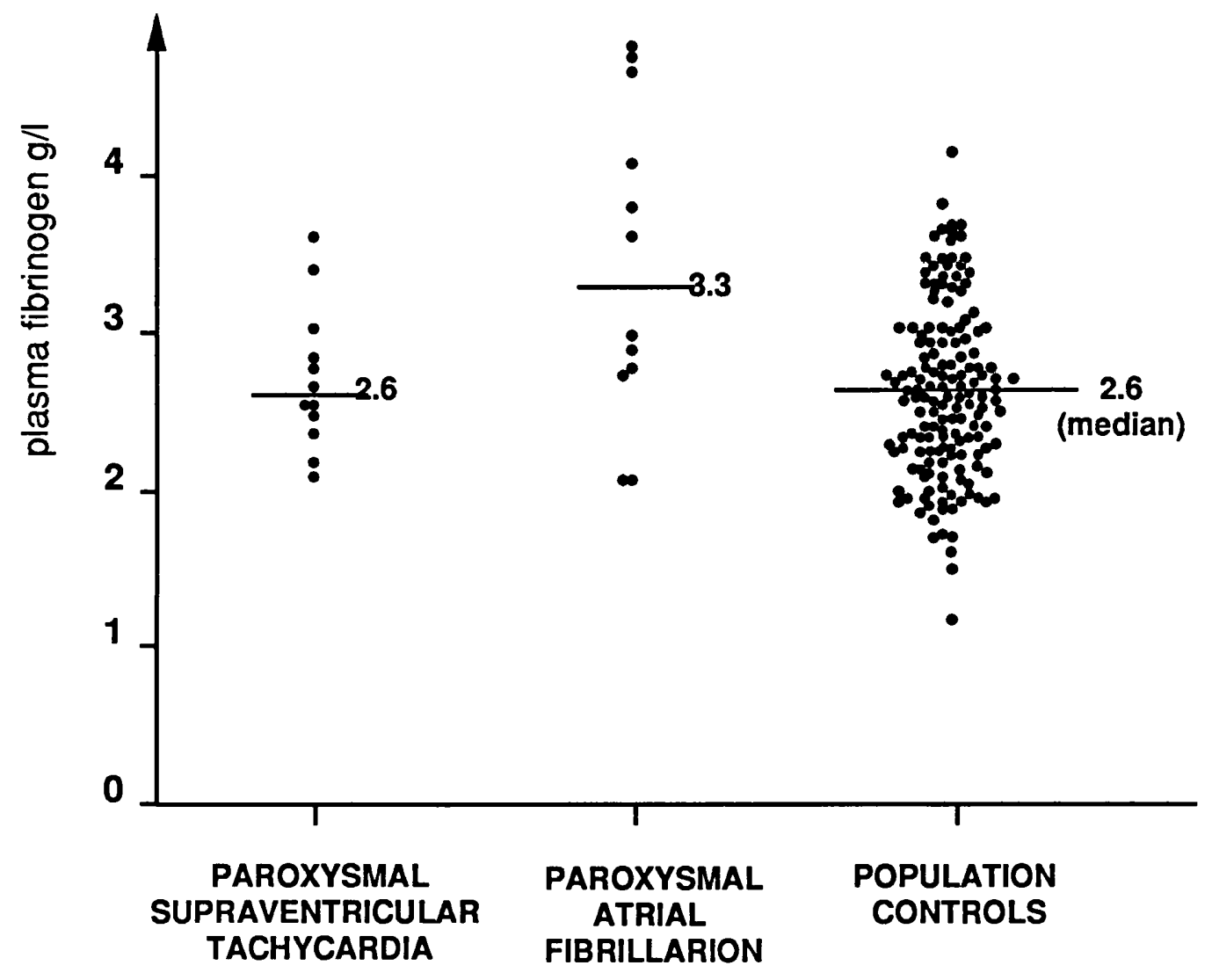

FIGURE 6.1

PLASMA FIBRINOGEN AND PAROXYSMAL SUPRAVENTRICULAR TACHYCARDIA 


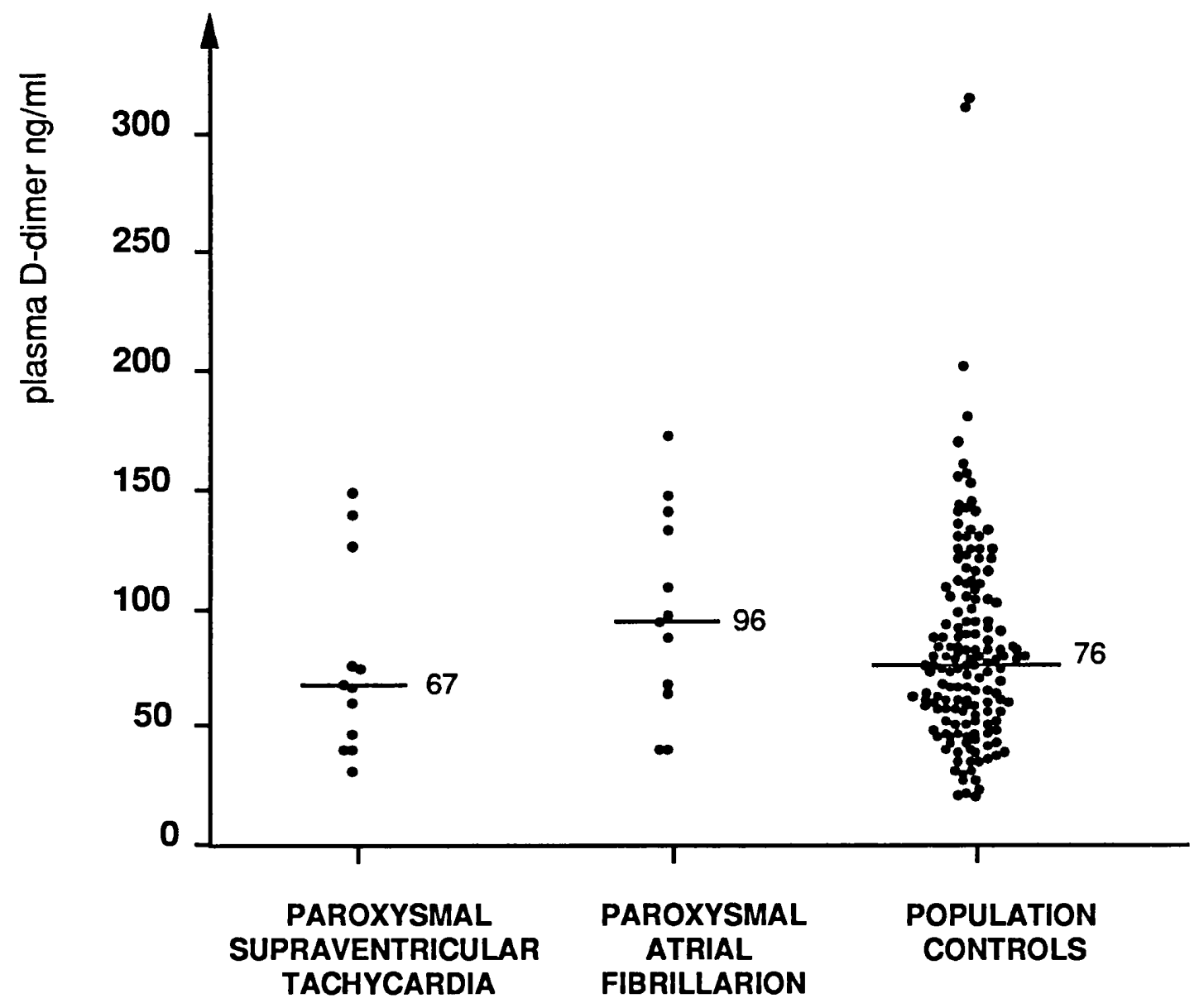

FIGURE 6.2

PLASMA D-DIMER AND PAROXYSMAL SUPRAVENTRICULAR TACHYCARDIA 
CHAPTER 7

THROMBOGENESIS
AND VALVE DISEASE 


\subsection{THE INFLUENCE OF HEART VALVE DISEASE ON THROMBOEMBOLIC RISK}

Introduction

In Chapter 3 of this thesis, an increase of plasma factors of thrombogenesis was demonstrated in a heterogeneous group of patients with atrial fibrillation which included those with ischaemic heart disease, rheumatic valvular disease and cardiomyopathy. The role of ischaemic heart disease will be addressed in Chapter 8 of this thesis, in which patients with coronary artery disease will be investigated in relation to cardiac function. A variety of other cardiac disorders can, however, be frequently found in patients diagnosed as having thromboembolic events and stroke (Hofmann et al 1990, Nishide et al 1983). This chapter will address the influence of underlying valve disease.

Rheumatic heart disease is a common echocardiographic finding in patients with embolic cerebrovascular disease (Hofmann et al 1990, Nishide et al 1983). In patients with valve disease who suffer an initial embolus, recurrent embolism occurs in $30-75 \%$ of patients, an annual rate of almost 10\% (Sherman et al 1990). Indeed, significant valvular stenosis and regurgitation may contribute to abnormalities of blood flow sufficient to promote thrombogenesis. Patients with aortic stenosis, for example, have marked alterations in blood flow through the stenosed valve (a high velocity jet demonstrable on continuous or pulsed wave Doppler echocardiography and visible as turbulence on colour Doppler). In addition, there may be secondary impaired compliance (and diastolic dysfunction) with left ventricular hypertrophy.

Echocardiography is a useful investigation in the assessment of patients with thromboembolism. The yield of echocardiography in identifying potential cardioembolic causes of stroke and other thromboembolic events varies widely in the literature depending upon the patient population and criteria of a cardioembolic source. Using restricted criteria, about $10 \%$ (range $4 \%$ to $16 \%$ ) of patients with ischaemic stroke will have a potential embolic source by echocardiography (Sherman et al 1989). Similarly, in a prospective study of 153 patients with arterial embolic events, transthoracic echocardiography was normal in only $60 \%$, whilst after both transthoracic and transoesophageal echocardiography only $42 \%$ had normal 
findings (Hofmann et al 1990). Of those with abnormal examinations in this study, a large proportion (25\%) had valvular heart disease (Hofmann et al 1990). It should be noted however that the definition of pathological valvular heart disease is increasingly difficult, as technology becomes more sensitive (Houston 1993). For example, there is an increase in the detection of valvular regurgitation with age, but this may be physiological (Houston 1993).

\section{Mitral valve disease}

The presence of mitral stenosis has a well-recognised increased thromboembolic risk, with an association of systemic embolization in $9-20 \%$ of patients (most of whom (60-75\%) have cerebral emboli) (Cohen et al 1993, Sherman et al 1990). The most important contributing factor to mitral stenosis in terms of risk is coexistent atrial fibrillation, which increases the risk of embolism from 3 to 7 times that of solitary mitral stenosis (Sherman et al 1990). Further evidence for this was noted in a study in which $16 \%$ of 622 patients with mitral stenosis and atrial fibrillation had left atrial thrombi identified by echocardiography, compared to only $1 \%$ of 192 patients with mitral stenosis in sinus rhythm (Beppu et al 1984).

The position with mitral regurgitation is less clear. Mitral valve prolapse has been reported as a cause of cerebral embolism and this diagnosis has to be considered especially in younger patients (Alpert 1993, Bogousslavsky and Regli 1987, Nishide et al 1983). These patients are often asymptomatic, and abnormal auscultatory findings are infrequent (Bogousslavsky and Regli 1987). Abnormalities of platelets and thrombosis may be the underlying cause of stroke in these patients. For example, thrombi have been detected on mitral valve leaflets of patients with mitral valve prolapse who have died of cerebral embolism (Lewis 1988). Abnormalities of platelet survival and increased platelet coagulant activity have also been shown in these patients (Lewis 1988), although a more recent study (23 patients) suggested the converse, with no differences in betathromboglobulin (a marker of platelet activation) demonstrable in patients with mitral valve prolapse (Lin et al 1989). However, it should be noted that mitral valve prolapse is often regarded as a heterogeneous diagnosis, resulting from diverse pathogenic mechanisms of the mitral valve apparatus (Braunwald 1993), which may in part account for the differences 
seen. The role of mitral regurgitation therefore in decreasing or increasing (as in mitral valve prolapse) any risk of thromboembolism remains unclear. Some evidence for a rheological mechanism may be obtained by considering the phenomenon of spontaneous echo contrast.

Spontaneous echo contrast refers to dynamic smoke-like echoes seen occasionally in the left atrial cavity during transthoracic echocardiography, but more commonly seen on transoesophageal echocardiography. The significance of spontaneous echo contrast has already been discussed in Chapter 3 of this thesis. Briefly, the mechanism of its formation is thought to be due to erythrocyte aggregation in low shear rate conditions, with a possible interaction of platelets and plasma fibrinogen. The latter is intimately involved in the rheological characteristics of blood flow. The presence of spontaneous echo contrast is associated with conditions favouring stasis of left atrial blood (for example, severe mitral stenosis), and appears to be a marker of previous thromboembolism in patients with nonvaluular atrial fibrillation and those with mitral valve disease or mitral valve replacement (Black al 1991). Importantly, the presence of left atrial spontaneous echo contrast has been found to be highly specific (94\%) and predictive for thromboembolic events (Tsai et al 1992).

In a large series (400 patients), mitral regurgitation was an independent predictor of the absence of left atrial spontaneous echo contrast, as only $7 \%$ of patients with moderate to severe mitral regurgitation were found to have the finding (Black et al 1991). Similarly, in two smaller series those with left atrial spontaneous echo contrast were less likely to have moderate or severe mitral regurgitation present (Chen et al 1990, Tsai et al 1992). These studies are however in contrast to an earlier study of 150 patients where there was a positive association between the presence of spontaneous echo contrast and mitral regurgitation (Castello et al 1990). The discrepancy may reflect differences in the patients studied, with a higher proportion of patients with mitral valve prostheses in the study by Castello et al (1990). From the rheological viewpoint, the presence of mitral regurgitation may preclude the development of spontaneous echo contrast by a 'stirring effect' on left atrial blood, thus eliminating blood stasis (Beppu et al 1985, Chen et al 1990). 
In addition, mitral regurgitation appears to protect against left ventricular thrombus in patients with dilated cardiomyopathy (Maze et al 1989). The development of left atrial thrombus postoperatively after mitral valve surgery for preoperative severe mitral regurgitation (which perhaps made the preoperative left atrial blood 'less stagnant') has also been reported (Iga et al 1993). If mitral regurgitation 'reduces' the formation of spontaneous echo contrast (and therefore, any rheological 'disturbance') and also protects against intracardiac thrombus formation, the presence of mitral regurgitation may therefore be a protective factor against thrombus formation. The situation is however far from clear. A study of prothrombotic markers may, however, provide the answer, and this is undertaken in this chapter. For the present, the indications for antithrombotic therapy in patients with mitral valve disease have been suggested as follows (Meltzer et al 1986): atrial fibrillation, patients in sinus rhythm with a left atrium larger than $55 \mathrm{~mm}$ (by echocardiography), a history of previous systemic embolization, mechanical prosthetic heart valves and bioprosthetic heart valves (usually for the first 3 months after implantation but indefinitely if atrial fibrillation or a dilated left atrium are present).

Aortic valve disease

The presence of aortic valve calcification and thickening is associated with systemic embolism (Hart 1992, Meltzer et al 1986, Nishide et al 1983). This is pertinent as calcification and degeneration of the aortic valve becomes more prevalent with increasing age. For example, in the Helsinki Ageing Study, some degree of valvular calcification was found in $28 \%$ of those aged $55-71$ years, with the prevalence rising to $75 \%$ of those aged 85 to 86 years (Lindroos et al 1993). In the absence of atrial fibrillation, however, calcific aortic (and mitral) stenosis are relatively uncommon causes of embolic stroke (Cohen et al 1993, Kapila and Hart 1986, Rancurel et al 1989). This is despite the demonstration of fibrin-platelet deposits on disrupted valvular endothelium, and the observation of calcific retinal emboli in patients with aortic stenosis (Sherman et al 1990). Calcific aortic stenosis may also be a source of embolization complicating cardiac catheterisation (Cohen et al 1993). 
Patients with aortic stenosis may develop left ventricular hypertrophy and heart failure (due to 'pressure overload'), and (as will be discussed in chapters 8 and 9) heart failure adds to the risks of thromboembolism. The presence of left ventricular hypertrophy (in association with aortic stenosis) in these patients can significantly contribute to diastolic dysfunction (Villari et al 1992). For example, diastolic dysfunction was present, despite normal systolic function, in 9 of 18 patients $(50 \%)$ of patients with aortic stenosis ('pressure overload') and 20 of 22 patients with aortic regurgitation ('volume overload') (Villari et al 1992). Indeed, it is increasingly recognised that diastolic dysfunction may be a common feature in valuular heart disease, and is independent of any coexisting systolic dysfunction (Hess et al 1991). In such patients, diastolic dysfunction is characterised by an impaired isovolumetric relaxation, an enhanced early diastolic filling rate and an increased myocardial stiffness (Hess et al 1991).

\section{Discussion}

Echocardiography has permitted an improved assessment of the heart in patients with thromboembolism and stroke, facilitating the diagnosis of cardiogenic embolism. It is now accepted, for example, that structural heart disease may dramatically increase the risk of thromboembolism in atrial fibrillation. However, it remains uncertain whether or not the presence of valvular heart disease in patients who are in sinus rhythm constitutes a significant thromboembolic risk (perhaps related to haemorheological abnormalities). Some answers may be provided by a study of possible haemorheological and prothrombotic factor abnormalities in two groups of such patients: those with mitral valve regurgitation (who are associated with flow abnormalities discussed above) and aortic stenosis. 


\subsection{STUDIES OF PLASMA FIBRINOGEN AND D-DIMER IN MITRAL REGURGITATION AND AORTIC STENOSIS}

Introduction

In various chapters ( 3 and 8 ) of this thesis, I have demonstrated that plasma fibrinogen is elevated for all groups of patients with chronic atrial fibrillation (irrespective of antithrombotic therapy) and coronary artery disease. One influencing factor in the assessment of these prothrombotic factors may be the presence of structural heart disease. This aspect has been addressed in this thesis for patients with chronic atrial fibrillation (chapter 3), whereby there was no significant difference in plasma fibrinogen or D-dimer levels for patients with or without structural heart disease. This finding is consistent with results of other investigators (Kumagai et al 1990). In contrast epidemiological evidence, from the Framingham study and other clinical studies, does suggest that the presence of rheumatic heart disease increases the thromboembolic risk of patients with chronic atrial fibrillation by approximately 18-fold (Cairns and Connolly 1991, Koenig et al 1992).

All valvular lesions may not have similar haemorheological effects and thromboembolic risks. For example, as previously discussed in section 7.1, the haemodynamic effects of valvular regurgitation may not be similar to that of valvular stenosis. This is supported by a lower incidence of spontaneous echo contrast (usually associated with blood stasis and thromboembolism) (Black et al 1991) and a reduction in left ventricular thrombus in patients with cardiomyopathy (Maze et al 1989) with associated mitral regurgitation. The presence of other valve lesions such as aortic valve disease may also provide an additional risk for thromboembolism but this has not been established.

The exact mechanisms for thrombus formation in patients with structural heart disease have not been clearly defined. The aim of this study therefore was to determine the effects of valvular heart disease (mitral regurgitation, aortic stenosis) on plasma fibrinogen or Ddimer levels as indices of a prothrombotic state. 


\section{Materials and methods}

The design of this study was a prospective population controlled study of male and female patients with valve disease in sinus rhythm. Patients were identified at the outpatient clinic or following routine cardiac catheterisation. I selected patients in sinus rhythm for further study if they had clinically demonstrable aortic stenosis or mitral regurgitation (the severity of the valve lesion was defined at echocardiography, as described later). Exclusion criteria were similar to previous studies and included those patients aged over 75 and those with significant systemic illnesses, hypertension, chronic infections or neoplastic disease. Patients with limited venous access, requiring transfusion or actively bleeding were also excluded. In addition, patients with significant coronary artery disease (defined at coronary angiography as the presence of one or

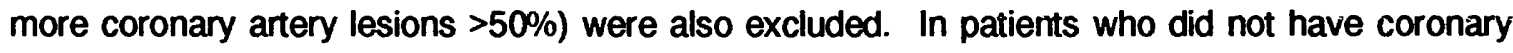
angiography, a history of ischaemic heart disease (previous myocardial infarction, angina or a positive exercise stress test) were grounds for exclusion from the study.

All patients had echocardiography performed to confirm the presence of structural heart disease (2-dimensional, M-Mode and Doppler), using a Hewlett-Packard Sonos 500 machine machine and techniques described in chapter 3. Patients with significant (moderate to severe) mitral regurgitation were identified from Doppler echocardiography by subjectively assessing the area of colour flow regurgitation jet in relation to the left atrial area (Helmcke et al 1987) (Figure 7.1); and by the presence of a dense continuous wave Doppler spectral signal with a peak transmitral flow velocity of $>4.0 \mathrm{~cm} / \mathrm{s}$. In addition the presence of mitral stenosis was assessed by reviewing 2-dimensional and M-mode echocardiographic appearances and assessment of the mitral valve pressure half-time. The presence of significant mitral stenosis was defined as patients with a mitral valve pressure half-time greater than 100 seconds, and these patients were excluded from further analysis. Patients with aortic stenosis were defined from the transaortic valve gradient demonstrated by continuous wave Doppler (Figure 7.2). An aortic valve gradient of $235 \mathrm{~mm} \mathrm{Hg}$ was considered indicative of significant aortic valve stenosis.

Methods for blood sampling and assays for plasma fibrinogen and fibrin D-dimer have been described previously (chapter 3). Results for patients were compared to 158 patients aged 55-65 years, sampled from a random local population study of healthy subjects (North 
Glasgow MONICA Study) which gave 'population' values. The sensitivity and reproducibility of the assays have been previously described. Detailed relationships were not examined for left atrial and ventricular sizes as other chapters have investigated this in detail.

All the values of the prothrombotic factor measurements were expressed as median \pm interquartile range (IQR). Statistical comparison was performed using the Mann-Whitney two sample rank test for non-parametric distributions and $95 \%$ confidence intervals (c.i.) calculated for the point estimate of the difference in medians. Correlations between clinical variables or rheological measurements were performed using Spearman's Rank correlation method. A probability of $\leq 0.05$ was considered statistically significant. Stepwise multiple regression analysis and multiple regression analysis was performed using plasma fibrinogen and D-dimer as dependent variables and age, body mass index, smoking, and the presence of mixed mitral valve disease as predictors in patients with mitral regurgitation. For patients with aortic stenosis, the age of the patient, body mass index, smoking, sex and aortic valve gradient were used as predictors in the analyses.

Results

A total of 25 patients with valve disease in sinus rhythm were studied: 12 patients (all female; mean age 55 years, s.e.m. 3.3) with mitral regurgitation (4 with mixed mitral valve disease, but with mild mitral stenosis (MV $\left.P_{1 / 2}<100\right)$ ); and 13 patients ( 7 male, 6 female; mean age 57 years, s.e.m. 3.4) with aortic stenosis ( 3 with mixed aortic valve disease) were studied. Patient characteristics are summarised in Table 7.1. Eight (5 male, 3 female) of these patients had coronary angiography as a preoperative assessment - none of these patients had significant coronary artery lesions.

Patients with mitral regurgitation had a median plasma fibrinogen which was significantly elevated when compared to female population values (point estimate of difference between medians $0.62 \mathrm{~g} /$; $95 \%$ c.i. 0.27 to $1.05, \mathrm{p}=0.0016$ ) (Figure 7.3). However, these patients had a median plasma D-dimer which was lower than that for population controls (point estimate of difference between medians $21 \mathrm{ng} / \mathrm{ml} ; 95 \%$ c.i. 0 to 38 , $\mathrm{p}=0.05$ ) (Figure 7.4). 
Patients with aortic valve disease had a median plasma fibrinogen which was significantly increased when compared to population controls (point estimate of difference between medians $0.82 \mathrm{~g} / \mathrm{l} ; 95 \%$ c.i. 0.34 to $1.24, p=0.001$ ) (Figure 7.3 ). These patients had a plasma fibrin D-dimer level which was similar to population values (point estimate of difference between medians $3 \mathrm{ng} / \mathrm{ml}$; 95\%c.i. -25 to 22, $\mathrm{p}=0.80$, NS) (Figure 7.4).

In patients with mitral regurgitation, stepwise multiple regression analysis with plasma fibrinogen and D-dimer as dependent factor demonstrated no significant influence of age, smoking habit, body mass index or the presence of mixed mitral valve disease on these plasma factors. There were no significant correlations between plasma fibrinogen or D-dimer and age or body mass index in these patients (Table 7.1). In patients with aortic stenosis, stepwise multiple regression analysis with plasma fibrinogen and plasma fibrin D-dimer as dependent factors demonstrated no significant influence of age, sex, smoking habit, body mass index or aortic valve gradient on these plasma factors. There were also no significant correlations between plasma fibrinogen or D-dimer and age, body mass index or aortic valve gradient in these patients (Table 7.1)

\section{Discussion}

This study demonstrates increased levels of plasma fibrinogen in both patient groups studied (mitral regurgitation and aortic stenosis). Both conditions are associated with abnormalities of blood flow and the elevation in plasma fibrinogen may be a reflection of rheological abnormalities present in these patients. However, the relationship to any thromboembolic risk is uncertain. The high plasma fibrinogen may, for example, reflect underlying coronary heart disease, although I have attempted to exclude such patients from this study. The elevation of plasma fibrinogen is not, however, due to an excess of smokers in the patients with valve disease when compared with population controls (Table 7.1).

The clinical significance of the altered plasma fibrinogen and D-dimer levels in these patients is uncertain, but may be related to flow (or haemorheological) abnormalities. Patients with mitral regurgitation exhibit abnormalities of blood flow due to the regurgitant 'jet' (although not the abnormalities associated with increased spontaneous echo contrast), and 
plasma fibrinogen changes may reflect this. The presence of mitral regurgitation may preclude the development of spontaneous echo contrast and intracardiac thrombus by a 'stirring' effect on left atrial blood (as discussed in section 7.1). As the presence of spontaneous echo contrast reflects stasis (and is associated with thromboembolism) its absence in patients with mitral regurgitation may account for the lack of elevation of [and a trend (reaching borderline significance) towards lower] plasma D-dimer levels in these patients. My findings are also in keeping with the majority of the literature suggesting a lower thromboembolic risk for patients who do not exhibit spontaneous echo contrast. Further studies associating the presence (or absence) of spontaneous echo contrast with changes in plasma fibrinogen and fibrin D-dimer are required however to fully answer this hypothesis. My findings are also consistent with the lower prevalence of left ventricular thrombus formed in patients with dilated cardiomyopathy and mitral regurgitation (Maze et al 1989).

Similarly to those patients with mitral regurgitation, patients with aortic stenosis also have increased plasma fibrinogen levels. This finding may again reflect flow abnormalities, visible (with colour Doppler) as a high velocity jet through the narrowed aortic valve orifice. It may be speculative to suggest that rheological abnormalities may partially contribute to the symptoms of syncope or presyncope in these patients. However, my study was small, with difficulties in patient recruitment; and only a small range of aortic valve gradients was studied. Further studies of patients with significant aortic stenosis are therefore required, with closer study of other rheological indices, such as plasma or blood viscosity and haematocrit.

Interestingly, patients with aortic stenosis had plasma fibrin D-dimer levels which were similar to control population levels. Intravascular fibrin turnover is, therefore, no different in these patients when compared with population levels and is consistent with the reported low incidence of thromboembolism in patients with aortic stenosis. However, thromboembolic events are associated with aortic stenosis, and the mechanisms of thrombogenesis in these patients may be different from that of atrial fibrillation and (as shown in chapter 8) left ventricular dysfunction. For example, fibrin-platelet deposits (demonstrated on disrupted valvular endothelium), and emboli from calcific aortic stenosis appear to be the 
principal pathological processes for emboli associated with aortic stenosis (Sherman et at 1990). This finding suggests that a different pathophysiological mechanism for emboli formation in aortic stenosis exists to that intimately associated with rheological factors (involving instead platelets and calcific emboli). This is possibly why no change in D-dimer levels is observed. In addition, the value of anticoagulation therapy in preventing these calcific emboli has not been established and the finding of 'normal' plasma D-dimer levels in this group of patients is consistent with this.

In conclusion, patients with mitral regurgitation or aortic stenosis have higher plasma fibrinogen levels when compared to 'normal' population values, suggesting possible rheological abnormalities in these patients. However, subjects with mitral regurgitation have lower plasma D-dimer levels suggesting lower intravascular clotting, consistent with clinical echocardiographic studies. Subjects with aortic stenosis have plasma D-dimer levels similar to the 'normal' population values, consistent with a different pathophysiological mechanism for the thromboembolic risk in these patients. These findings add to an improved understanding of the relationship between clinical and echocardiographic observations and the significance of plasma fibrinogen and fibrin D-dimer levels in thrombogenesis. 
TABLE 7.1

PLASMA FIBRINOGEN AND D-DIMER IN

\section{MITRAL REGURGITATION AND AORTIC STENOSIS}

(a) patient characteristics and results:

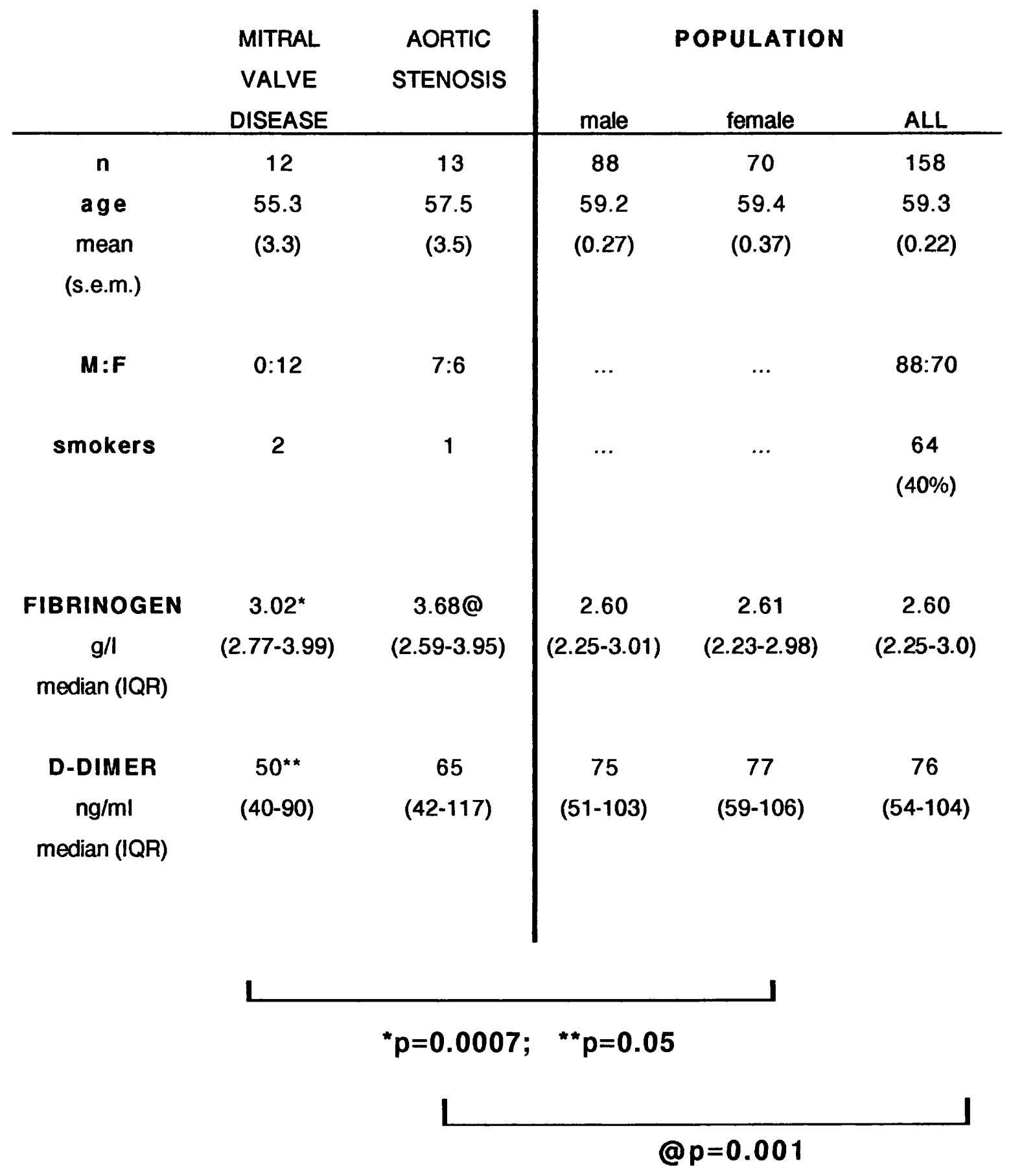


(b) Spearman correlations for plasma fibrinogen and D-dimer:

\begin{tabular}{|c|c|c|c|c|}
\hline \multirow[b]{2}{*}{$\begin{array}{c}r \\
\text { (p value) }\end{array}$} & \multicolumn{2}{|c|}{ mitral regurgitation } & \multicolumn{2}{|c|}{ aortic stenosis } \\
\hline & fibrinogen & D-dimer & fibrinogen & D-dimer \\
\hline fibrinogen & $\cdots$ & $\begin{array}{c}0.31 \\
(0.327)\end{array}$ & $\cdots$ & $\begin{array}{c}0.21 \\
(0.53)\end{array}$ \\
\hline D-dimer & $\begin{array}{c}0.31 \\
(0.327)\end{array}$ & $\ldots$ & $\begin{array}{c}0.21 \\
(0.53)\end{array}$ & $\ldots$ \\
\hline Age & $\begin{array}{c}0.31 \\
(0.324)\end{array}$ & $\begin{array}{c}0.33 \\
(0.303)\end{array}$ & $\begin{array}{c}0.35 \\
(0.23)\end{array}$ & $\begin{array}{c}0.26 \\
(0.58)\end{array}$ \\
\hline $\begin{array}{l}\text { Body mass } \\
\text { index }\end{array}$ & $\begin{array}{c}0.48 \\
(0.14)\end{array}$ & $\begin{array}{c}0.21 \\
(0.52)\end{array}$ & $\begin{array}{l}-0.46 \\
(0.13)\end{array}$ & $\begin{array}{l}-0.18 \\
(0.60)\end{array}$ \\
\hline $\begin{array}{l}\text { Aortic valve } \\
\text { gradient }\end{array}$ & $\cdots$ & $\cdots$ & $\begin{array}{l}-0.24 \\
(0.57)\end{array}$ & $\begin{array}{c}0.06 \\
(0.85)\end{array}$ \\
\hline
\end{tabular}




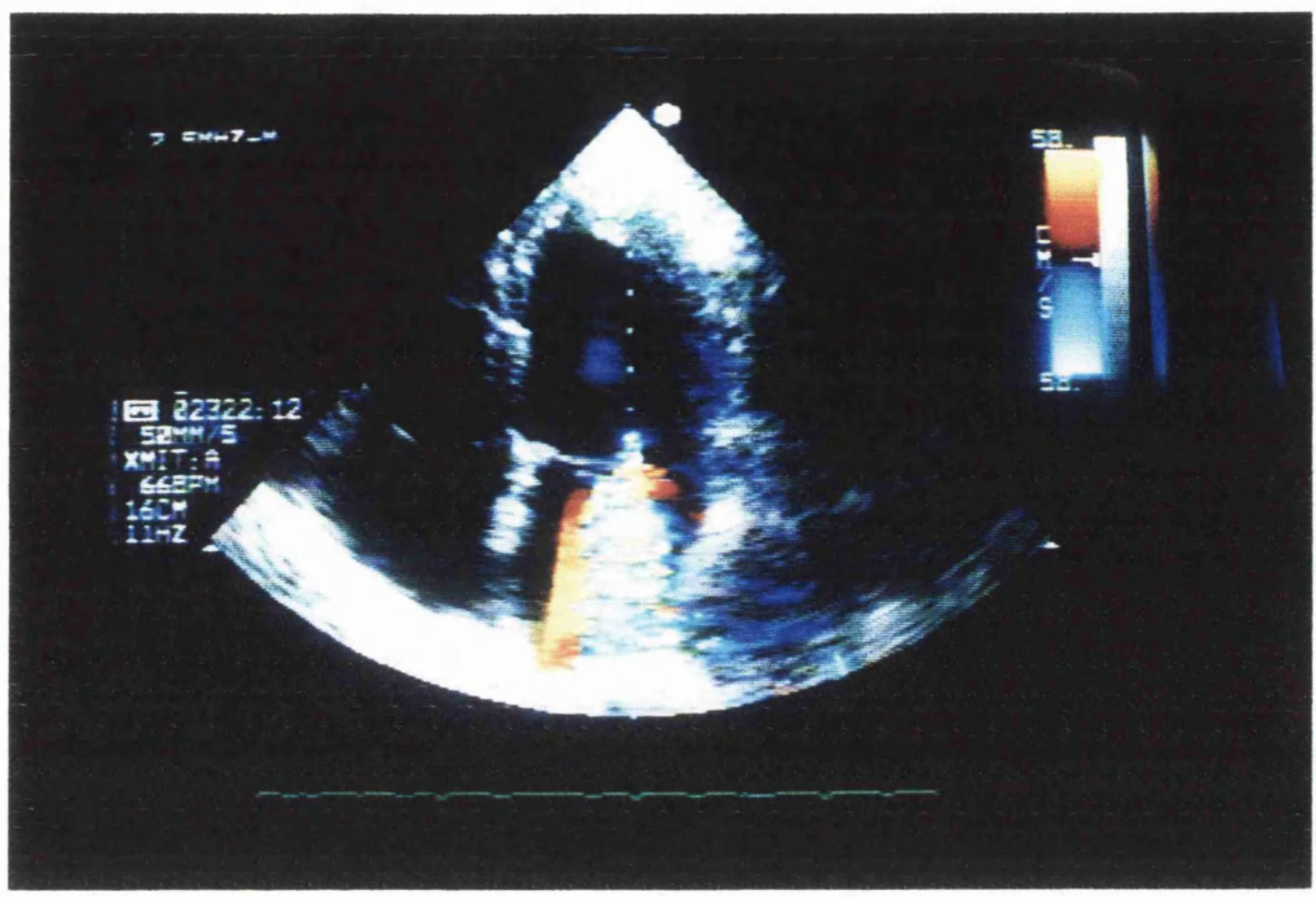

FIGURE 7.1

AN EXAMPLE OF COLOUR FLOW DOPPLER ECHOCARDIOGRAPHY demonstrating mitral regurgitation 


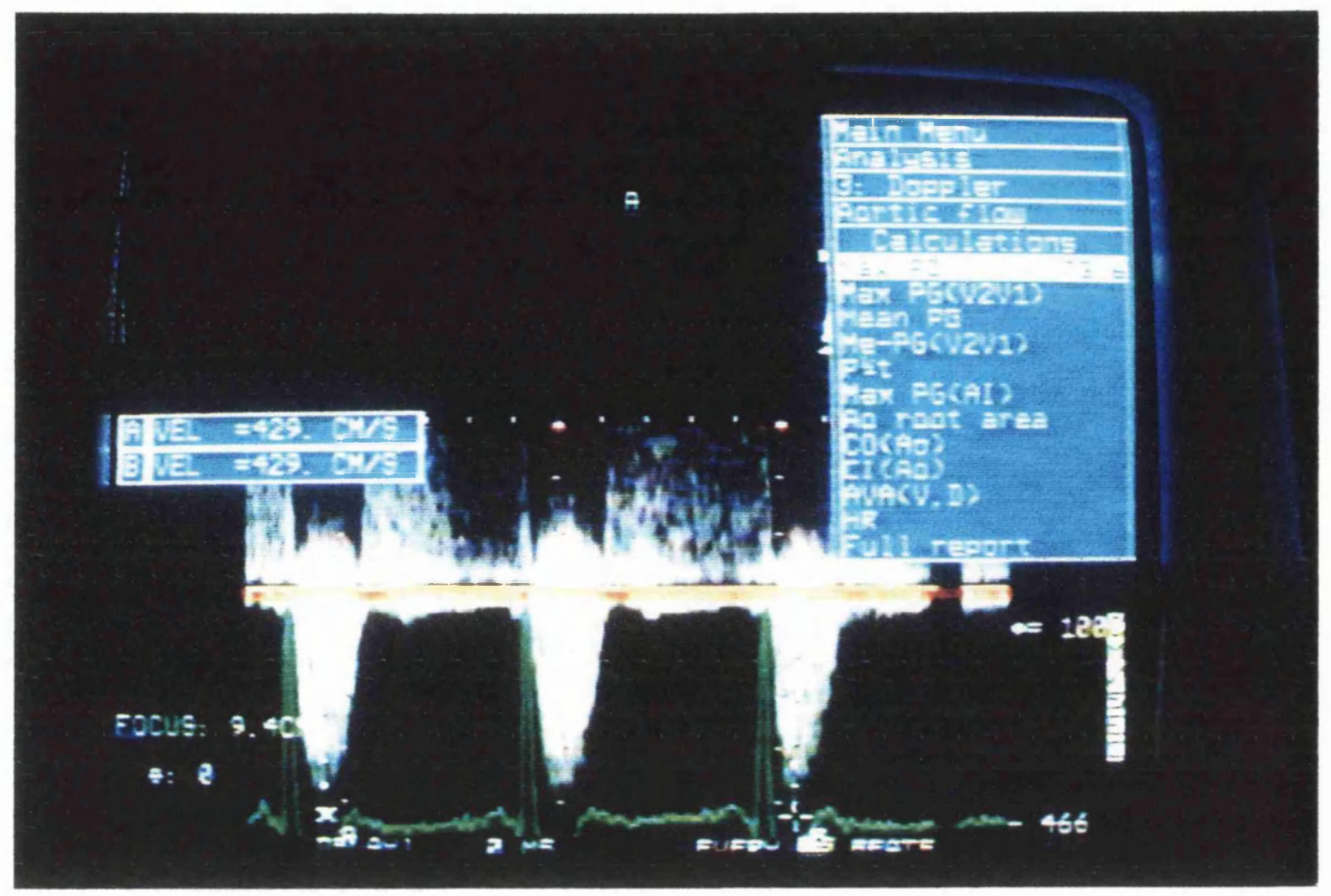

Aortic Regurgitation

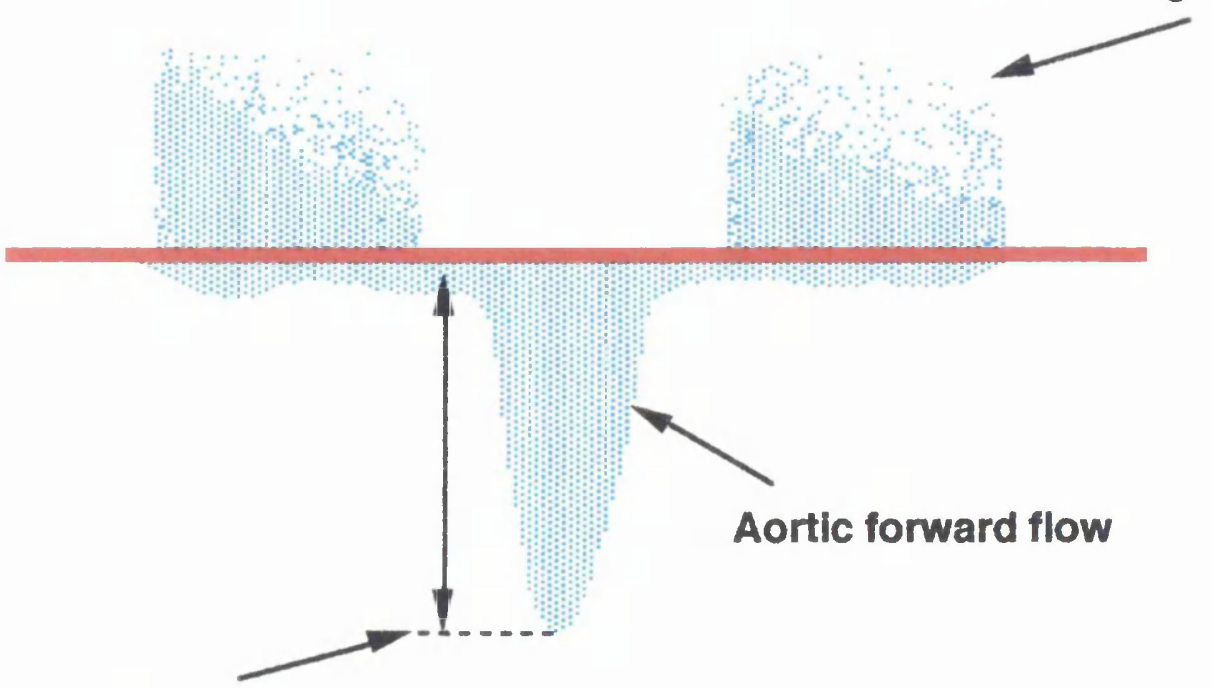

Maximum transaortic valve velocity (v)

[Maximum aortic valve gradient $\simeq 4 \vee^{2}$ ]

FIGURE 7.2

AN EXAMPLE OF CONTINUOUS WAVE DOPPLER ECHOCARDIOGRAPHY

demonstrating mixed aortic valve disease

(with an aortic valve gradient of $73 \mathrm{mmHg}$ and associated mild aortic regurgitation) 


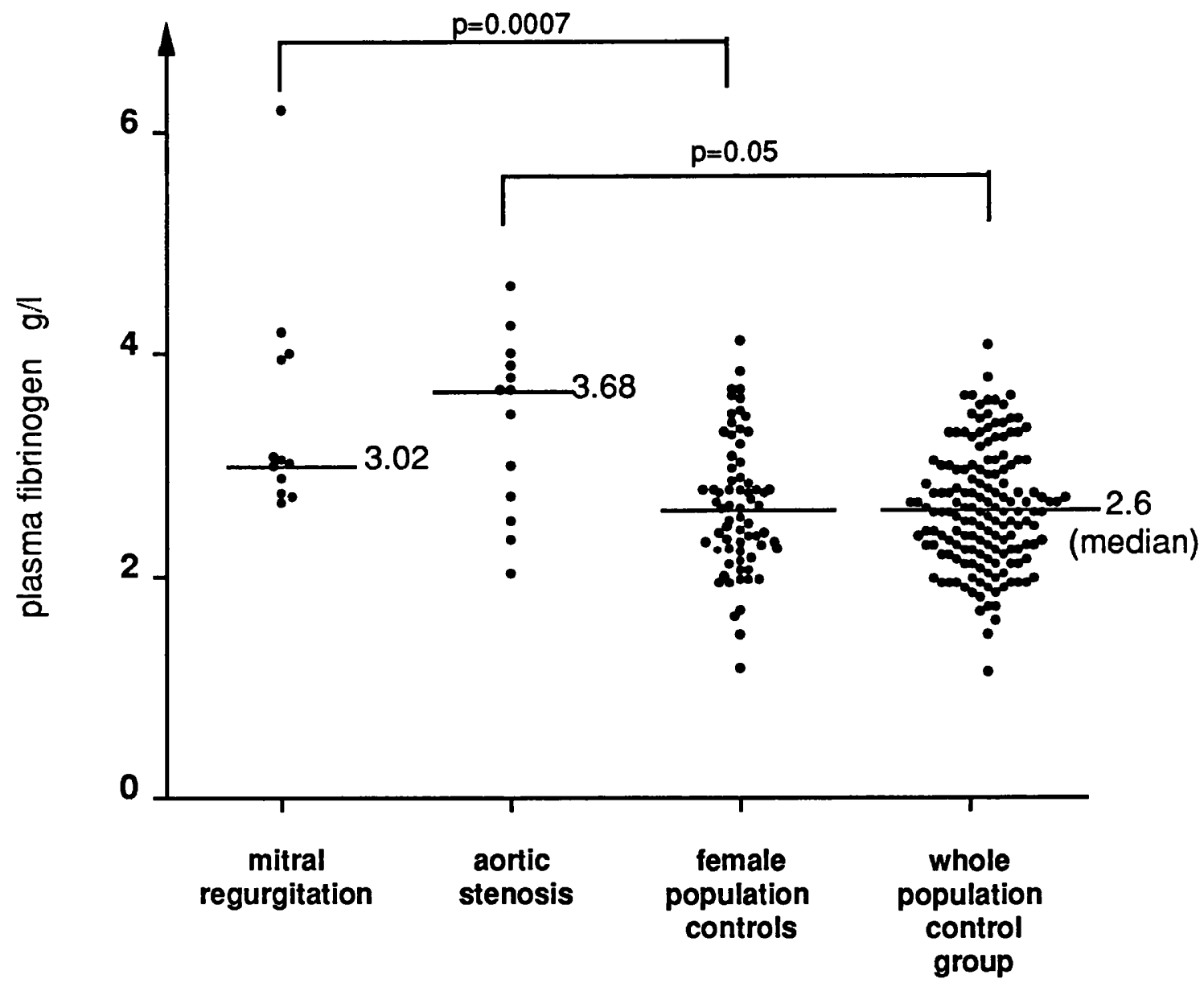

FIGURE 7.3

PLASMA FIBRINOGEN AND VALVE DISEASE 


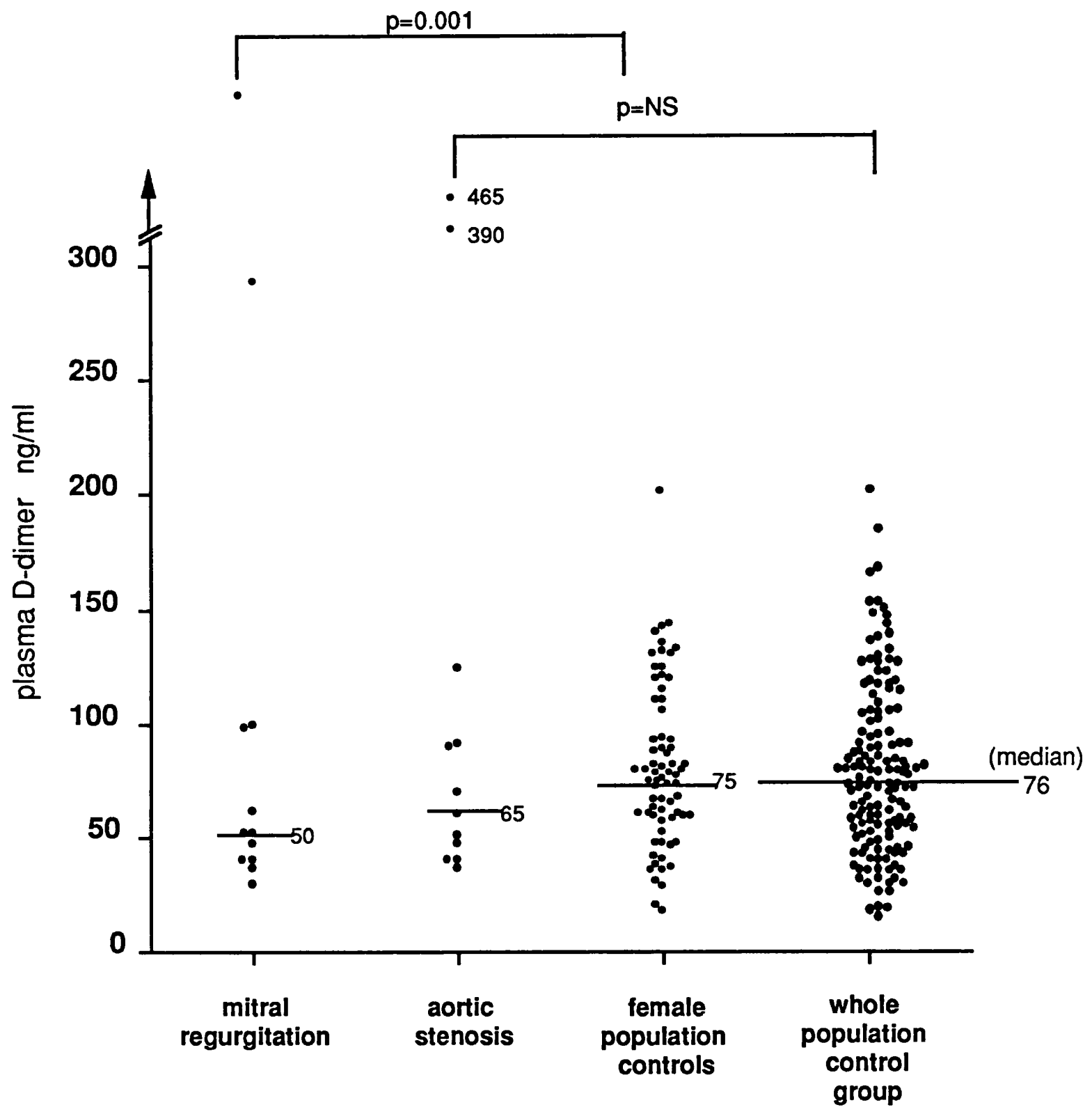

FIGURE 7.4

PLASMA D-DIMER AND VALVE DISEASE 
CHAPTER 8

\section{THROMBOGENESIS IN \\ LEFT VENTRICULAR SYSTOLIC \\ DYSFUNCTION}




\subsection{IS SYSTOLIC DYSFUNCTION IMPORTANT IN THROMBOGENESIS?}

\section{Introduction}

Patients with impaired left ventricular function are at high risk of intracardiac thrombus and thromboembolism. The presence of left ventricular dysfunction is also an additive risk factor to the risk of stroke and thromboembolism in patients with atrial fibrillation (Hart 1992, Stroke Prevention in Atrial Fibrillation Investigators 1992a, Stroke Prevention in Atrial Fibrillation Investigators 1992b). In addition, it is well recognised that thromboembolism is a frequent cause of death in patients with heart failure (Griffith et al 1952). One specific group of patients with left ventricular dysfunction who appear to be at particular risk of intracardiac thrombus are patients with left ventricular aneurysm (Nixon 1983).

Overall, the incidence of left ventricular aneurysm following myocardial infarction is about 7 to $10 \%$ and surgical, autopsy and echocardiographic studies indicate that about half of all patients, with post-infarction ventricular aneurysm, have mural thrombus present (Cabin and Roberts 1980, Cregler 1992, Weinreich et al 1984). Despite the associated theoretical increase in thromboembolic risk, there is some evidence that these thrombi may only rarely embolise (Cregler 1992). The precise reasons for this are unclear, but the haemodynamic effects of left ventricular dysfunction or aneurysm may contribute to any altered thromboembolic risk in such patients.

Investigations

In patients with left ventricular systolic dysfunction, investigations are required to determine the degree of cardiac impairment, to diagnose the presence of aneurysm and finally, to detect the presence or absence of thrombus. Techniques employed in the assessment of left ventricular dysfunction include contrast cineangiography, echocardiography, and radionuclide methods.

Left ventricular contrast cineangiography may be useful for the diagnosis of significant wall motion abnormalities, including the presence of a left ventricular aneurysm. However, the investigation is less useful for the diagnosis of mural thrombus with a reported 
sensitivity of $31 \%$, a specificity of $75 \%$ and a predictive accuracy of only $54 \%$ (Reeder et al 1981).

Echocardiography, however, is widely available and relatively inexpensive. It is now the method of choice for detecting left ventricular thrombus, although the sensitivity of two-dimensional echocardiography in the detection of left ventricular thrombus varies between 77 to $95 \%$ with a specificity of 86 to $93 \%$ (Cregler 1992, Ezekowitz et al 1982, Stratton et al 1982, Visser et al 1983). About 15\% (with a quoted range of between $5 \%$ and 25\%) of studies are, however, technically inadequate and cannot exclude the presence of a thrombus (Cregler 1992, Ezekowitz et al 1982). Two-dimensional echocardiography may also have disadvantages in visualising the left ventricular apex for characteristic wall thinning and possible apical thrombus formation (seen in aneurysm formation), as the sector angle is 90 degrees and the apex is usually too near the probe in the 4-chamber view (Iga et al 1992). Overall cardiac motion towards the apex in systole also tends to accentuate apical dyskinesis and to obscure inferoposterior wall dyskinesia (Kessler 1990). A final limitation of echocardiography is its inability to reflect thrombus activity, that is, whether or not ongoing thrombogenesis is present. It is useful, however, in quantifying thrombus size and mass. Echocardiographic indications of left ventricular thrombi are summarised in Table 8.1.

Radionuclide techniques are increasingly used in the diagnosis of left ventricular dysfunction and the presence of intracardiac thrombus. For example, equilibrium gated radionuclide ventriculography, employing $99 \mathrm{~m}$ Technetium isotope, is useful as an objective measure of left ventricular function. It also allows assessment of wall motion abnormalities, particularly by the study of phase and amplitude images. In addition, one useful radionuclide method of identifying left ventricular thrombus employs indium-111 platelet scintigraphy (Ezekowitz et al 1982, Kessler et al 1987). This has a specificity of $100 \%$ and sensitivity of $71 \%$, which is even greater than that of echocardiography (Cregler 1992, Ezekowitz et al 1982, Vandenberg 1990). One further advantage of this method is its ability in assessing the surface activity of the thrombus, which may therefore complement any echocardiographic assessment. 
Other useful techniques for the investigation of left ventricular function and thrombus include magnetic resonance imaging (Higgins et al 1985, Iga et al 1992) and computed tomography (CT) (Nair et al 1981), but these techniques are limited to few centres.

\section{Left ventricular aneurysms}

The definition of what a left ventricular aneurysm is remains controversial and may vary considerably between different studies and with different investigational methods (Cabin and Roberts 1980). Obviously, if definitions vary from study to study, the results or conclusions may do so likewise; this may make some comparisons between the studies difficult to interprete.

It is generally accepted that the presence of a left ventricular aneurysm may result in significant haemodynamic consequences. This includes the reduction in stroke volume and ejection fraction, and the presence of life-threatening arrhythmias. All of these changes may result in increased susceptibility to congestive heart failure and a lower exercise tolerance (La Rovere et al 1987). The presence of such haemodynamic changes may have important implications for patient survival. In one series, for example, the survival rates of patients with ventricular aneurysm at 1, 3 and 5 years were 88,82 and $75 \%$ respectively (Heras et al 1992). In such patients, an increased left ventricular end diastolic pressure (>20 $\mathrm{mm} \mathrm{Hg}$ ), a greater number of diseased vessels and the presence of bifascicular block were independent predictors of high mortality (Faxon et al 1982, Heras et al 1992). Other predictors of mortality include age, clinical severity of heart failure, the left ventricular ejection fraction, the presence of left anterior descending artery obstruction and the presence or absence of coronary collaterals (Faxon et al 1982, Shen et al 1992). However it appears that the prognosis of this group of patients is primarily related to left ventricular dysfunction, whilst the presence of an aneurysm alone may not independently alter survival (Faxon et al 1982, Shen et al 1992).

Systemic embolism is a commonly cited complication of left ventricular aneurysm. Indeed, apical akinesis, dyskinesis or both appear to be a prerequisite for the development of thrombus (Weinreich et al 1984). Late thrombi are common in patients with 
congestive heart failure and deteriorating left ventricular systolic function, although there is some evidence that impaired left ventricular function on its own is not a prerequisite for thrombus formation (Weinreich et al 1984). This is complemented by evidence that the reduction in heart rate and left ventricular apical wall motion with the early use of beta-blocker therapy may actually increase the risk of thrombus formation (Johannessen et al 1988). Contrary to expectation, the introduction of thrombolytic therapy (streptokinase or recombinant tissue plasminogen activator) in the acute management of patients with anterior myocardial infarction does not appear to reduce the risk of left ventricular thrombus formation (Cregler 1992). Few data exist on whether early intervention with coronary angioplasty may reduce the risk of subsequent left ventricular thromboembolism (Cregler 1992).

Reported incidences of systemic embolism in patients with left ventricular aneurysm can vary widely - ranging from 0 to 52\% (Lapeyre et al 1985, Mullen et al 1977, Schlichter et al 1954). However, mural thrombus does appears to occur more frequently in the presence of an aneurysm (Schlichter et al 1954). One reason for this wide variation in incidence is the lack of uniformity in defining what an aneurysm is, as discussed above (Cabin and Roberts 1980). It is usually accepted that the identification of mural thrombus in patients with left ventricular aneurysm warrants long-term oral anticoagulation as part of any medical management strategy (Keating et al 1983, Nixon 1983, Reeder et al 1981, Sherman et al 1990). For example, in an overview of six studies (mean follow-ups 6 to 15 months), Sherman et al (1990) demonstrate that late emboli occurred in only $1 \%$ of 98 anticoagulated patients, when compared to $13 \%$ of 46 non-anticoagulated patients. It is also argued, however, that in chronic left ventricular aneurysms diagnosed at least six months after myocardial infarction, thrombus is common but the risk of embolism is low (an incidence of 0.35 per 100 patientyears) and that anticoagulation may not be warranted (Cabin and Roberts 1980 , LaPeyre et al 1985, Meltzer et al 1986).

The role of preventive strategies against thromboembolism in patients with left ventricular aneurysms therefore remains controversial, since the haemodynamic status of the patient may continue to alter with time. For example, aneurysm expansion may result in progressive haemodynamic deterioration with increasingly impaired left ventricular function. 


\section{Left ventricular dysfunction}

There is clinical evidence for a high thromboembolic risk in patients with impaired left ventricular function (but without aneurysm formation). For example, poor left ventricular function adds to the risk of stroke and thromboembolism in atrial fibrillation (Stroke Prevention in Atrial Fibrillation Investigators 1992a, Stroke Prevention in Atrial Fibrillation Investigators 1992b). In addition, thromboemboli are a frequent cause of death in patients with congestive heart failure, as shown in an postmortem study of 565 patients with heart failure, where evidence of thromboemboli was found in $30.3 \%$ (Griffith et al 1952). The mechanisms for this increased risk are uncertain, but poor systolic function may cause stasis of blood within the dilated left ventricular cavity resulting in mural thrombus formation (Meltzer et al 1986)

Thrombus has been found in approximately $36 \%$ of patients with chronic dilated cardiomyopathy on two dimensional echocardiography (Gottdiener et al 1983). Systemic emboli occurred in $11 \%$ of these patients, but there was no difference in the incidence of emboli when compared to those with no thrombus visualised at echocardiography (Gottdiener et al 1983). It is therefore felt that the embolic potential of left ventricular thrombus may be directly related to the presence of adjacent segments of normally functioning myocardium (with altered flow characteristics) and inversely related to the stability of the thrombus. For example, in an echocardiographic study of 40 patients with cardiomyopathy, there were specific abnormally low flow profiles at the ventricular apex associated with the presence of left ventricular thrombus (Maze et al 1989). Similar abnormal flow profiles have been demonstrated by pulsed Doppler echocardiography in patients post myocardial infarction who subsequently develop intracardiac thrombus (Delemarre et al 1990). In this latter study, the abnormalities were evident in diastole, suggesting that the absence of diastolic recoil of the apex of the heart was additive to the impaired systolic contraction in creating disturbed flow patterns (Delemarre et al 1990). This finding may explain the increased risk of intracardiac thrombus in patients with left ventricular aneurysms. Interestingly, in the cardiomyopathy patients without intracardiac thrombus, there was a higher 
prevalence of mitral regurgitation despite abnormal Doppler flow profiles (Maze et al 1989). The presence of mitral regurgitation may therefore serve as a protective factor against thrombus formation as the higher inflow velocities may result in a lower tendency for thrombus formation. The findings of these two studies therefore suggest that flow abnormalities are important in promoting thrombogenesis, and that Doppler echocardiography may be useful in patient risk stratification by the identification of a high risk group (with abnormal flow patterns), who would benefit from prophylactic anticoagulation (Kessler 1990).

Whether not all patients with poor left ventricular function should be treated with anticoagulants is debatable. There are few data advocating routine use of oral anticoagulant therapy in these patients. In one study (390 patients) there was a significant reduction in thromboembolism in patients with chronic heart failure, especially in those with coexisting rheumatic heart disease (Griffith et al 1952). More importantly, warfarin therapy is associated with a higher incidence of thrombus resolution at echocardiography when compared to patients on no therapy ( $59 \%$ vs $29 \%$ respectively) (Stratton et al 1988). In contrast to these findings, however, another echocardiographic study demonstrated that anticoagulant therapy provided protection against embolic events but the presence of thrombi on follow-up echocardiography at six months was the same regardless of treatment (Weinreich et al 1984). Further evidence comes from two series in which there was also no apparent effect of anticoagulation on the prevalence of mural thrombus, identified at aneurysmectomy (Reeder et al 1981, Simpson et al 1980). However, the duration of warfarin therapy correlated inversely with the presence of thrombus, and this consideration may account for the discrepancy in thrombus prevalence with warfarin therapy in the different studies (Reeder et al 1981).

An argument against the use of routine anticoagulant therapy is the finding that the presence of left ventricular thrombus on echocardiography may not be predictive of systemic emboli (Gottdiener et al 1983). Although the prevalence of mural thrombi is high in left ventricular aneurysms, these thrombi only rarely embolise (Simpson et al 1980). The factors that may predict the likelihood of embolization of any left ventricular thrombus are therefore important. 
The morphology of a thrombus can often help to predict subsequent embolization. For example, thrombus protrusion and mobility, especially those related to a recent myocardial infarction, are associated with an increased embolic risk (Haughland et al 1984, Stratton et al 1988). The use of indium ${ }^{111}$ platelet scintigraphy may be helpful in identifying patients with chronic left ventricular thrombi at increased risk of embolisation. In one study, embolization occurred in $23 \%$ of patients with a positive platelet scintigram and echocardiogram, when compared with embolization in only $4 \%$ of patients with a negative platelet scintigram but positive echocardiogram (Stratton and Ritchie 1990). This method may therefore detect 'active' thrombi, which are at high risk of embolisation, and ongoing thrombogenesis may account for the positive platelet scintigraphy in these patients. Measurement of a suitable plasma marker of ongoing thrombogenesis (for example, plasma Ddimer) may complement this investigation.

The situation is therefore far from clear - just who is at risk of thromboembolism? Suggested guidelines for initiating anticoagulant therapy in patients with left ventricular thrombi are summarised in Table 8.2. For a mural thrombus to embolise, a large portion of its surface must be unattached to the underlying wall. For example, in patients sustaining a large anterior myocardial infarction with poor cardiac function, a protruding, mobile left ventricular thrombus requires anticoagulant therapy. The identification of patients at risk of systemic embolization is of particular importance - echocardiography and/or platelet scintigraphy may be useful, but the availability of a simply measured plasma marker of thrombogenesis might allow stratification of risk and assist in the management of such patients. Further discussion on the role of anticoagulation is to be found in chapter 10 of this thesis. 
TABLE 8.1

\section{ECHOCARDIOGRAPHIC INDICATIONS OF}

LEFT VENTRICULAR THROMBI (adapted from Meltzner et al 1986)

Mass of echoes in the left ventricular cavity with a well circumscribed, defined border.

Abnormal motion pattern of the underlying myocardium, usually dyskinetic.

Distinct border shown between underlying myocardium and thrombus.

Echocardiographic 'texture' different from that of underlying myocardium.

Usually apical position.

Ability to be recorded from more than one position.

TABLE 8.2

INDICATIONS FOR ANTICOAGULATION THERAPY FOR POSSIBLE LEFT VENTRICULAR THROMBI

(adapted from Meltzner et al 1986)

Left ventricular aneurysm 3 months or more after acute myocardial infarction - usually no need

for echocardiography or anticoagulation treatment, unless established heart failure

Dilated cardiomyopathy

- treatment regardless of presence or absence of left ventricular thrombi.

Acute myocardial infarction

- large infarction, with associated congestive cardiac failure.

- anterior myocardial infarction without heart failure, especially if left ventricular thrombi are found on 2-D echocardiogram. 


\subsection{STUDIES OF FIBRINOGEN, VON WILLEBRAND FACTOR AND FIBRIN D- DIMER IN LEFT VENTRICULAR SYSTOLIC DYSFUNCTION.}

Introduction

Clinical evidence has demonstrated that the presence of heart failure significantly contributes to the thromboembolic risk of patients with chronic atrial fibrillation. This was confirmed in the Stroke Prevention in Atrial Fibrillation study, where congestive heart failure was one of three independent clinical predictors of thromboembolism, the others being a history of hypertension and previous thromboembolism (Stroke Prevention in Atrial Fibrillation Investigators 1992a). In addition, left ventricular dysfunction seen on the 2-D echocardiogram appeared to be a strong predictor of later thromboembolic events (Stroke Prevention in Atrial Fibrillation Investigators 1992b). Patients with left ventricular dysfunction (especially those in whom a left ventricular aneurysm is present) are therefore thought to be at an increased thromboembolic risk despite being in sinus rhythm (Nixon 1983). The exact mechanism for thrombus formation in left ventricular aneurysms has not been clearly defined but may involve contributions from poor left ventricular function, increased stasis within a dilated left ventricle (or within the area of the aneurysm), and possibly, additional alterations of flow characteristics within the heart. Poor systolic function in an impaired ventricle may therefore result in sufficient stasis within a dilated left ventricular cavity to promote thrombus formation (Meltzer et al 1986). A high prothrombotic state may therefore exist in these patients, with a high degree of intravascular thrombogenesis.

As I have demonstrated in chapter 3 of this thesis, plasma fibrinogen is elevated in all groups of patients with chronic atrial fibrillation, irrespective of antithrombotic therapy. An attempt to correlate plasma levels of these haemorheological and prothrombotic factors with left ventricular function in this study of patients with chronic atrial fibrillation did not demonstrate any significant associations. The measurement of left ventricular function in the patients with chronic atrial fibrillation was based, however, on fractional shortening, which may not be an accurate assessment of left ventricular dysfunction especially in the presence of large wall motion abnormalities. 
The aim of this study was therefore to specifically determine the effects of left ventricular aneurysm formation and left ventricular dysfunction upon plasma fibrinogen and fibrin D-dimer levels. This would possibly provide evidence for a prothrombotic state with increased intravascular fibrin turnover or thrombogenesis in patients with left ventricular systolic dysfunction.

\section{Materials and Methods}

The design was a prospective population controlled study of male and female patients with coronary artery disease. Patients were initially selected following routine cardiac catheterisation. I selected patients for further study if they were felt to have either normal left ventricular contraction or a significant wall motion abnormality on left ventriculography. Exclusion criteria were identical to that in chapter 3 . Results for patients were also compared to 158 patients aged 55-65 years, sampled from a local population study (North Glasgow MONICA Study) as in chapter 3. In addition, as all patients undergoing cardiac catheterisation were on aspirin therapy, I also included a population of patients with documented ischaemic heart disease (previous myocardial ischaemia or infarction) with normal cardiac function (no history of cardiac failure and normal echocardiography), but were not on aspirin therapy due to drug allergy or intolerance (for example, peptic ulcer disease). This group served as hospital controls.

Assessment of left ventricular function All patients had left ventricular function initially assessed by review of the dynamic single-plane left ventriculogram (performed at cardiac catheterisation). A consensus opinion of 2 observers for cardiac function as seen on the left ventriculogram was obtained before inclusion. Patients with possible wall motion abnormalities were then further assessed by echocardiography and by a radionuclide ventriculogram (RNVG).

Radionuclide ventriculography was performed using a low energy general purpose collimator following in vivo red cell labelling with $99 \mathrm{~m}$-technetium pertechnetate. Sixteen frames per cycle were obtained using a frame-mode algorithm with exclusion of 'bad beats', acquiring 200,000 counts per frame. Two projections were imaged (the standard $40^{\circ}$ left anterior oblique view (which may be slightly adjusted to visualise the best view of the interventricular septum) and a left lateral view) and used for subsequent wall motion analysis. Analysis of the left 
ventricular ejection fraction was performed using the 'best septal' left anterior oblique view. The left ventricular region of interest was carefully delineated to exclude the left atrium, pulmonary veins and right ventricle throughout the cardiac cycle. After the background region of interest was chosen, a time-activity curve was generated. Left ventricular ejection fractions were then calculated by computer-analysis of the time-activity curves, normalised to background counts. In our laboratory, the normal left ventricular ejection fraction was considered $\geq 40 \%$. This equilibrium gated study gave an objective measurement of left ventricular ejection fraction and categorisation of wall motion. A left ventricular aneurysm was therefore defined from the RNVG by the presence of a paradoxical wall motion abnormality. This was determined by abnormal phase (within the atrial range), presence of amplitude but evidence of a reversed stroke volume in the aneurysm region.

Echocardiography All of these patients had echocardiography performed primarily to assess for the presence or absence of intracardiac thrombus and also for the presence of wall thinning in aneurysm formation. The presence of a left ventricular aneurysm was identified by echocardiography as the presence of a regional wall motion abnormality with diastolic distortion and systolic dyskinesis. As in chapter 3, two dimensional and Doppler echocardiography was performed with the patient in the left lateral decubitus position using a Hewlett-Packard Sonos 500 machine (Hewlett-Packard, Arondale, Pa, USA.) equipped with a standard $2.5 \mathrm{MHz}$ transducer. Standard images were obtained, with the transducer was angled to maximise cardiac chamber size and gain positions were adjusted to obtain the clearest outline of the endocardium. Off line analytical methodology was used to quantify measurements of left atrial dimensions and left ventricular dimensions (similar to that in chapter 3). Left atrial volume was calculated using a standard ellipsoid formula (Sanfillipo et al 1990). The thickness of the septum and posterior wall, and left ventricular dimensions were measured using M-mode echocardiography in the parasternal long axis view (Sahn et al 1978). Left ventricular fractional shortening was calculated and the ejection fractions were estimated for those without significant wall motion abnormalities. The echocardiographic left ventricular ejection fraction (EF) was also calculated (with standard software on the echocardiography machine) using the method of Teichholz et al (1976), which was derived from the equation EF $=1-E S V / E D V$, and involved the calculation of ventricular 
volumes at end-diastole (EDV) and end-systole (ESV) [using the formula: $V=[7 / 2.4+D] D^{3}$, where $\mathrm{V}=$ volume, and $\mathrm{D}=$ the echocardiographically measured internal dimension].

The interobserver and intraobserver variability for 2-D and M-mode echocardiographic measurements have been described in chapter 3. A sample of 20 patients selected at random were studied twice on different occasions by the same observer (myself) and also by one other observer. Detailed Doppler echocardiography allowed assessment of the role of diastolic dysfunction and this is discussed in detail in chapter 9 .

Plasma fibrinogen, von Willebrand factor and D-dimer assays Plasma fibrinogen, von Willebrand factor (WWF) and fibrin D-dimer were assayed using techniques previously described in chapter 3.

Statistical methods All the values of the prothrombotic factor measurements were expressed as median \pm interquartile range (IQR). Statistical comparison was performed using the KruskalWallis test for multiple comparisons and Mann-Whitney two sample rank test for non-parametric distributions. For parametric distributions, oneway ANOVA and student's paired t-test were used. Correlations between clinical and echocardiographic measurements and rheological values were performed using Spearman's Rank correlation method. A probability of $\leq 0.05$ was considered statistically significant.

Results

A total of 112 patients (89 male and 23 female; mean age 57.5 years, s.e.m. 0.86) with coronary artery disease documented by coronary angiography were studied. At the time of the study, 34 patients had normal left ventricular function (Group 1), 30 had left ventricular dysfunction without aneurysm formation (Group 2) and 29 had left ventricular aneurysm (Group 3a). In addition, 19 patients with left ventricular aneurysms were established on warfarin therapy (Group 3b). These patients on warfarin had Thrombotest results within the therapeutic range (515\%). As patients were all on aspirin therapy, results for patients in Group 1 were compared to 24 hospital controls (14M, 10F; mean age 57.8 , s.e.m. 2.35) who were patients with ischaemic heart 
disease in sinus rhythm and normal cardiac function who were not on aspirin therapy due to contraindications such as dyspepsia and gastrointestinal intolerance. All the results were also compared to 158 population controls [subjects aged 55-65 years, sampled from a local population study (North Glasgow MONICA Study; described earlier]. Clinical variables in these groups are as shown in Table 8.3 There were no differences in age (one way ANOVA $F=0.28, p=0.84$ ) and body mass index (one way ANOVA $F=1.08, p=0.362$ ) between the groups. Similarly there were no differences in sex ratio $\left(X^{2}=3.6,3\right.$ d.f.; $\left.p=0.31\right)$, proportion of smokers $\left(X^{2}=1.35,3\right.$ d.f.; $\mathrm{p}=0.72$ ) or severity of coronary artery disease (that is, proportions with single-, two- or threevessel disease; $X^{2}=6.32,6$ d.f; $p=0.39$ ) between the groups studied.

Plasma fibrinogen, von Willebrand factor and D-dimer levels Plasma fibrinogen (median 3.18g/ IQR 2.64-3.58) and D-dimer (median 58ng/ml, IQR 34-88) levels were measured in all patients, but von Willebrand factor was only assayed in 95 patients from Groups 1,2 and 3a (median 124\%, IQR 93 to 172). As a group, all patients had significantly elevated plasma fibrinogen levels when compared to population controls (point estimate of difference between medians $0.53 \mathrm{~g} / \mathrm{l} ; 95 \%$ C.I. 0.38 to $0.67, \mathrm{p}<0.0001$ ). Plasma vWF levels were also increased when compared to population controls (point estimate of difference between medians $18 \%$; $95 \%$ C.I. 6 to 31, $p=0.005$ ). There were significant differences in plasma fibrinogen (Kruskal-Wallis test $H=50.5,4$ d.f.; $p<0.0001$ ), D-dimer (Kruskal-Wallis test $H=33,4$ d.f. $p<0.0001$ ) and vWF (Kruskat Wallis test $H=12.3,3$ d.f.; $p=0.007$ ) between the groups (Groups $1,2,3 a$ and $3 b$ ).

In Group 1 (Normal LV function), plasma fibrinogen (point estimate of difference between medians $0.36 \mathrm{~g} / \mathrm{l} ; 95 \%$ C.I. 0.15 to $0.57, p=0.0009$ ) and vWF (point estimate of difference between medians $17 \%$; $95 \%$ C.I. 1 to $33, p=0.04$ ) was significantly elevated when compared to population controls (Table 8.4). There was a lower plasma D-dimer level in these patients when compared to population controls (point estimate of difference between medians $23.0 \mathrm{ng} / \mathrm{ml}$; $95 \%$ C.I. 9 to $36, p=0.001$ ) (Table 8.4). There were no significant differences in plasma fibrinogen (point estimate of difference between medians $-0.16 \mathrm{~g} / \mathrm{l} ; 95 \%$ C.I. -0.53 to $0.15, p=N S$ ) or fibrin D-dimer (point estimate of difference between medians $8.25 \mathrm{ng} / \mathrm{ml} ; 95 \%$ C.I. -33 to $10, p=N S$ ) when compared to hospital controls (Table 8.5). 
In Group 2 (LV dysfunction but no aneurysm), there was no significant difference in plasma fibrinogen (point estimate of difference between medians $0.14 \mathrm{~g} / \mathrm{l} ; 95 \%$ C.I. -0.23 to $0.44, p=N S$ ), vWF (point estimate of difference between medians $10 \% ; 95 \%$ C.I. -13 to $13, p=$ NS) or D-dimer levels (point estimate of difference between medians $0 \mathrm{ng} / \mathrm{ml}$; $95 \%$ C.I. -7 to 29 , $p=N S$ ) in these patients when compared to Group 1 (Table 8.4).

In Group 3a (LV aneurysm without warfarin), plasma fibrinogen was significantly elevated when compared to Group 1 (point estimate of difference between medians $0.6 \mathrm{~g} / ;$; $95 \%$ C.I. 0.23 to $1.0, p=0.0001$ ). In these patients, plasma D-dimer was also significantly elevated when compared to Group 1 (point estimate of difference between medians $55 \mathrm{ng} / \mathrm{ml} ; 95 \%$ C.I. 23 to $103, p=0.0007$ ). There was a trend towards higher vWF levels in Group 3a compared to Group 1 (point estimate of difference between medians $24 \%$; $95 \%$ c.i. -53 to $8, p=0.13$ ). In similar patients on warfarin therapy (Group 3b), there was no significant difference in plasma fibrinogen (point estimate of difference between medians $0.39 \mathrm{~g} /$; $95 \% \mathrm{C.I} .-0.04$ to $0.91, \mathrm{p}=\mathrm{NS}$ ), but there was a significantly lower plasma fibrin D-dimer level when compared to Group 3a (no warfarin therapy) (point estimate of difference between medians $65.5 \mathrm{ng} / \mathrm{ml} ; 95 \%$ C.I. 28 to 132 , $p=0.0003$ ) (Table 8.4).

Radionuclide ventriculography and echocardiography data All patients in Group 2 and $3 a$ had equilibrium radionuclide ventriculography performed. However, two patients in Group 2 and one from Group 3a had uninterpretable RNVGs due to multiple extrasystoles. Only 15 patients from Group 1 were referred for RNVG but normal left ventricular function was established for all members of this group by both a normal left ventriculogram at cardiac catherisation and normal echocardiography. As expected, patients in Group 2 and 3 had lower ejection fractions by RNVG (median ejection fractions $28 \%$ and $29 \%$ respectively) and echocardiography (median ejection fractions $43 \%$ and $40 \%$ respectively) when compared to patients in Group 1 ( $47 \%$ by RNVG, 55\% by echocardiography).

Mural thrombus was only visualised in one patient by echocardiography; a 38 year male who also had an recent cerebrovascular event. His prothrombotic markers were 
high, with plasma fibrinogen, vWF and D-dimer levels of $4.48 \mathrm{~g} /, 192 \%$ and $131 \mathrm{ng} / \mathrm{ml}$ respectively.

Effects of cardiac chamber size and ventricular function on plasma fibrinogen, D-dimer and vWF levels.

In Group 1 (normal left ventricular function) there were significant correlations between plasma fibrinogen and left ventricular fractional shortening $(r=-0.43, p=0.023)$ and echocardiographically-derived ejection fraction ( $r=-0.42, p=0.02)$; and $\mathrm{VWF}$ and left ventricular end-diastolic dimension $(r=0.43, p=0.026)$. (Table 8.7) Stepwise multiple regression analysis and multiple regression analysis with plasma fibrinogen, D-dimer and vWF as dependent factors demonstrated no significant predictors amongst age, sex, smoking habit, ejection fraction (RNVG), body mass index, left atrial volume and left ventricular fractional shortening (fibrinogen F 2.77, $p=0.17$; D-dimer $F 1.74, p=0.53$; vWF $F=0.51, p=0.81$ ).

In Group 2 (left ventricular dysfunction), there were significant correlations between plasma $D$-dimer and age $(r=0.57, p=0.001)$, left ventricular end-diastolic dimension $(r=0.418, p=0.04)$ and left atrial dimension $(r=0.472, p=0.007)$. In addition, there were correlations between vWF and left atrial dimension $(r=-0.44, p=0.013)$ and left atrial volume $(r=-0.37, p=0.04)$ (Table 8.7). Stepwise multiple regression analysis and multiple regression analysis with plasma fibrinogen, D-dimer and vWF as dependent factors demonstrated no significant predictors amongst age, sex, smoking habit, ejection fraction (RNVG), body mass index, left atrial volume and left ventricular fractional shortening (fibrinogen $F$ 1.22, $p=0.36$; Ddimer $F 2.0, p=0.16$; vWF $F=0.74, p=0.66$ ).

In Group 3 (aneurysms) there were no significant correlations between plasma fibrinogen, vWF and D-dimer with cardiac chamber size or cardiac function. Stepwise multiple regression analysis and multiple regression analysis with plasma fibrinogen, D-dimer and VWF as dependent factors demonstrated no significant predictors for D-dimer and VWF amongst age, sex, smoking habit, ejection fraction (RNVG), body mass index, left atrial volume and left ventricular fractional shortening (D-dimer $F=0.7, p=0.70$; vWF $F=0.69, p=0.69$ ). For plasma fibrinogen, however, smoking was a significant predictor $(t=2.29, p<0.05)$ using 
stepwise multiple regression analysis, although multiple regression with the same variables demonstrated no significant predictors $(F=0.70, p=0.699)$.

\section{Discussion}

This study demonstrates that as a group, patients with coronary artery disease have significantly elevated plasma fibrinogen and von Willebrand factor (vWF) levels when compared to random population sample controls of similar age in sinus rhythm. This is in keeping with epidemiological evidence of an association between plasma fibrinogen, vWF and ischaemic heart disease. However, some differences emerge when these patients are subdivided on the basis of cardiac function. Patients with left ventricular aneurysms, for example, have the highest levels of plasma fibrinogen, vWF and D-dimer. These findings are not due to an excess of smokers, nor are they attributable to an increase in the extent of coronary artery disease in view of similar proportions of patients with 2 or 3 vessel disease in the groups studied (Table 8.3) .

As epidemiological and clinical evidence suggests that elevated plasma fibrinogen levels are associated with increased cardiovascular mortality and morbidity, this finding would suggest that these patients are at increased cardiovascular risk. In addition, these patients have higher plasma wWF levels when compared to population controls. This is pertinent as an elevated VWF level is associated with an increased risk of reinfarction and subsequent mortality in survivors of myocardial infarction (Jansson et al 1991). Patients with left ventricular aneurysms have the highest levels of plasma vWF, which may be explained by two possible mechanisms. Firstly, they would appear to have the greatest endothelial dysfunction (as reflected by vWF levels), potentially leading to the highest intravascular thrombogenesis (consistent with the highest plasma D-dimer levels being found in these patients). Secondly, patients with the highest vWF levels have the greatest cardiovascular risk and consequently these patients may sustain the largest myocardial infactions, resulting in the most cardiac 'damage' (and aneurysm formation). Further prospective studies examining the predictive effects of high plasma vWF for cardiovascular events and thromboembolism in healthy population surveys are required to assess these hypotheses. 
The high plasma fibrin D-dimer in patients with left ventricular aneurysms suggests ongoing intravascular fibrin turnover in these patients. In addition, the increased plasma fibrinogen levels may promote thrombogenesis in these patients either by increasing the tendency towards thrombosis or by increasing flow disturbance through rheological effects by increasing red cell aggregation and blood viscosity in areas of stasis. My findings in this study (and the possible haemorheological explanation) is in keeping with experimental evidence demonstrating regional stasis of blood in hearts with severe apical dysfunction (Mikell et al 1982). In an aneurysm, there is both diastolic and systolic bulging, resulting in severe stasis of blood, with consequent predisposition towards thrombus formation. As abnormalities in blood flow predispose towards thrombus formation (as part of Virchow's triad), this study is the first haemorheological demonstration of abnormally enhanced fibrin turnover and thrombogenesis in patients with left ventricular aneurysms. As plasma fibrin D-dimer is a marker of intravascular thrombus formation, my findings are also consistent with the hypothesis that the more severe the degree of blood stasis, the more likely that intravascular thrombogenesis will occur.

In the setting of ischaemic heart disease, the risk of thrombus formation is proportional to the size of the infarct (and therefore the extent of wall motion abnormality). For example, stroke is a known complication of an anterior myocardial infarction, occurring in approximately $6 \%$ of patients (Cerebral Embolism Task Force 1989). Most cardiac thrombi form in close association to dyskinetic wall motion abnormalities and embolization is most common in the first 4 months (Weinreich et al 1984). Inferior myocardial infarction is less commonly a precursor of embolic stroke unless significant ventricular dysfunction is also present (Weinreich et al 1984). It has been noted that apical left ventricular aneurysms are usually associated with thrombus formation, but the embolic risk is usually smaller than that for other dyskinetic segments (Lapeyre et al 1985). However, transthoracic echocardiography is a sensitive and specific method only for the detection of mural thrombus of $>5 \mathrm{~mm}$ in diameter (Hildebrand et al 1993). For example, mural thrombus was detected by echocardiography in only one patient, who also had a recent cerebrovascular event. Echocardiography may therefore still miss smaller collections of thrombus that are still capable of producing 
devastating strokes and other serious thromboembolic complications. As measurement of plasma fibrin D-dimer is a marker of intravascular thrombus formation, elevated levels may indicate the presence of thrombus although not visualised at echocardiography. In contrast, patients with left ventricular aneurysms whom are established on oral anticoagulant therapy have significantly lower median plasma D-dimer levels, which is consistent with the prophylactic benefit of warfarin therapy in reducing thromboembolic risk.

The interaction between prothrombotic factors and cardiac structure or function is illustrated in this study. In Group 1, for example, plasma fibrinogen levels are negatively correlated with left ventricular fractional shortening and echocardiographicallyderived ejection fraction; and von Wilebrand factor is positively correlated with left ventricular end-diastolic dimension. This would suggest that those with poorer left ventricular function would have higher plasma fibrinogen levels and that endothelial dysfunction is greater in patients with dilated left ventricles, although these relationships are not seen in Group 2 or 3. In Group 2, however, there was a modest positive correlation between plasma D-dimer and age, left atrial size and left ventricular end-diastolic dimension, suggesting a relationship between intravascular thrombogenesis and age and dilated cardiac chambers. Again these associations are not seen in the other groups. Multiple regression analysis did not, however, show any significant predictors for plasma fibrinogen, vWF or D-dimer. The relationships between prothrombotic factors and cardiac structure and function is therefore complex, and in view of the differences between the subgroups, the relationships appear to be altered by the degree of overall cardiac function.

It is surprising that patients with ischaemic heart disease (and normal left ventricular function, Group 1) had significantly lower plasma D-dimer levels when compared to population controls. This may be in part explained by the use of aspirin therapy in Group 1, although the effect was not significant when Group 1 patients were compared to hospital controls (Table 8.5) or in patients with chronic atrial fibrillation on aspirin therapy (chapter 3). In addition, several subjects in the 'healthy' population controls may have (undiagnosed) underlying cardiovascular disease which may affect the levels of these prothrombotic factors. 
My findings in this chapter would therefore be compatible with the conclusions of chapter 3 for patients with chronic atrial fibrillation. Again, measurement of plasma fibrin D-dimer may identify a subgroup of patients, with left ventricular aneurysms (those with increased D-dimer), whom would benefit particularly from the introduction of oral anticoagulation therapy. This would be of clinical advantage as prophylactic anticoagulation continues to have two problems; inconvenience and safety (Lowe 1992). A prospective study of the effects of initiating oral anticoagulation therapy is required to test this hypothesis, and this will be further studied in chapter 10. 
TABLE 8.3

LEFT VENTRICULAR SYSTOLIC DYSFUNCTION - PATIENT CHARACTERISTICS

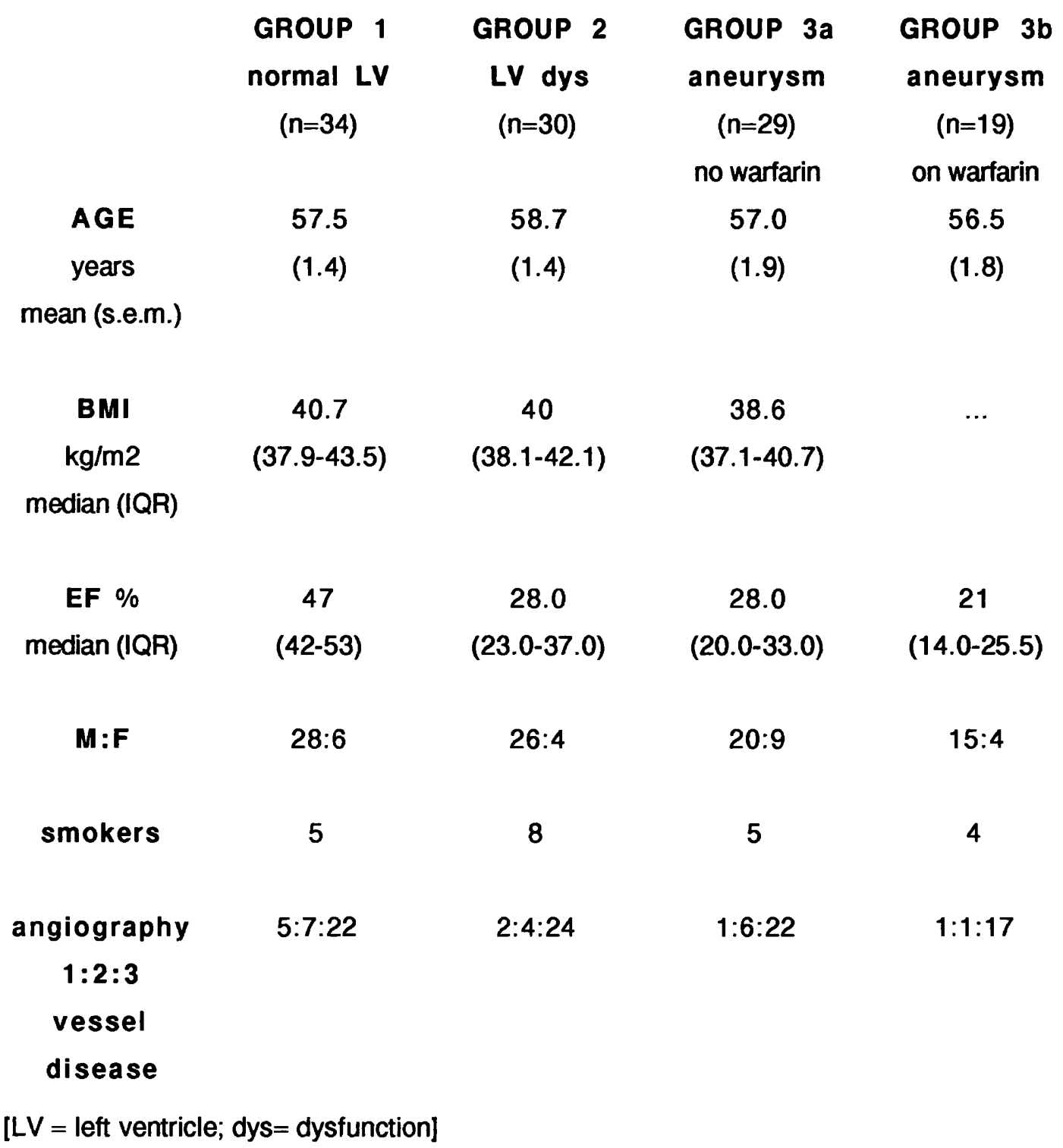




\section{TABLE 8.4}

\section{LEFT VENTRICULAR FUNCTION AND PROTHROMBOTIC FACTORS}

\begin{tabular}{|c|c|c|c|c|}
\hline $\begin{array}{c}\text { population } \\
\text { controls }\end{array}$ & $\begin{array}{c}\text { GROUP } 1 \\
\text { Normal LV }\end{array}$ & $\begin{array}{c}\text { GROUP2 } \\
\text { LV } \\
\text { dysfunction }\end{array}$ & $\begin{array}{c}\text { GROUP } 3 a \\
\text { ANEURYSM } \\
\text { no warfarin }\end{array}$ & $\begin{array}{c}\text { GROUP } 3 b \\
\text { ANEURYSM } \\
\text { warfarin }\end{array}$ \\
\hline
\end{tabular}

$\begin{array}{cccccc}\mathbf{n} & 158 & 34 & 30 & 29 & 19 \\ \text { FIBRINOGEN } & 2.6 & 2.97^{\star} & 2.73 & 3.50 @ & 3.24 \\ \text { median } & (2.5-3.0) & (2.65-3.35) & (2.34-3.30) & (3.00-4.40) & (2.89-3.61) \\ \text { g/l (IQR) } & & & & & \\ & & & & 101 @ @ & 57^{+} \\ \text {D-DIMER } & 76 & 48^{\star *} & 61.0 & (60-190) & (32-66) \\ \text { median } & (54-104) & (30-84) & (30-106) & & \\ \text { ng/ml (IQR) } & & & & 156 & \\ \text { von } & 105 & 123^{\star \star *} & 117 & (101-201) & \\ \text { Willebrand } & (80-147) & (100-156) & (76-161) & & \\ \text { factor } \\ \text { median } \\ \text { mg/l (IQR) }\end{array}$

$l^{*} \mathrm{p}=0.0004,{ }^{\star \star} \mathrm{p}=0.0015,{ }^{\star \star *} \mathrm{p}=0.0398$ vs population controls;

$@ p=0.002$, @@p=0.0001 vs Group 1;

$+p=0.0001$ vs Group 3a.] 


\section{TABLE 8.5}

\section{ASPIRIN AND PLASMA FIBRINOGEN \& D-DIMER LEVELS}

$\begin{array}{ccc} & \text { GROUP } 1 & \text { HOSPITAL CONTROLS } \\ & \text { Normal LV } & \text { IHD,SR } \\ \text { on aspirin } & \text { no aspirin } \\ \text { A GE } & 34 & 24 \\ \text { mean (s.d.) } & 57.8 & 57.8 \\ \text { FIBRINOGEN } & (8.1) & (11.5) \\ \text { median } & & \\ \text { g/I } & 2.96 & 3.07 \\ \text { median (IQR) } & (2.65-3.35) & (2.68-3.55) \\ & & \\ \text { FIBRIN D-DIMER } & & \\ \text { ng/mI } & & 63 \\ \text { median (IQR) } & (30-84) & (40-90) \\ & & \end{array}$

$p=N S$ 
TABLE 8.6

\section{LEFT VENTRICULAR SYSTOLIC DYSFUNCTION -ECHOCARDIOGRAPHY DATA}

$\begin{array}{cccc}\text { GROUP } 1 & \text { GROUP } 2 & \text { GROUP } 3 & \text { HEALTHY } \\ \text { Normal LV } & \text { LV dysfunction } & \text { LV aneurysm } & \text { CONTROLS }\end{array}$

n

33

30

29

14

\section{LEFT ATRIUM}

D1 $\mathrm{cm}$

$3.89^{\star \star}$

4.10

4.04

3.37

median (IQR)

(3.54-4.18)

(3.73-4.48)

(3.47-4.46)

(3.09-3.65)

D2 $\mathrm{cm}$

3.82

4.14@

4.01

3.67

median (IQR)

(3.5-4.11)

(3.72-4.34)

(3.68-4.39)

(3.45-3.89)

D3 cm

$5.13^{\star \star}$

5.25

5.18

4.61

median (IQR)

(4.67-5.50)

(4.81-5.67)

(4.75-5.64)

(4.20-4.79)

\section{LEFT ATRIAL \\ VOLUME \\ $\mathrm{cm}^{3}$}

median (IQR)

\section{LEFT}

VENTRICLE

ESD cm

3.6

$37.6^{\star \star}$
$(30.5-53.8)$

45.4

41.1

29.2

(34.4-55.9)

(32.9-59.4)

(26.2-31.5)

median (IQR)

(3.4-4.1)

4.4@@

$4.5^{\circ 00}$

3.4

median (QR)

EDD $\mathrm{cm}$

5.3

(4.0-4.8)

(3.6-5.7)

(3.2-3.6)

median (IQR)

(5.1-5.6)

5.8@@

$5.7^{\infty}$

5.2

\section{fractional}

$30.0^{*}$

(5.3-6.2)

(5.3-6.9)

(4.9-5.5)

shortening

(26.9-35.2)

22.1@@

$19.8^{\infty 00}$

34.5

$\%$

(16.4-26.9)

(15.1-27.2)

(30.9-37.3)

median (IQR) 
EDV

135

166@@

$163^{\circ}$

128

cm3

(124-150)

(134-193)

(130-242)

(111-147)

median (IQR)

ES V

55*

87@@

$99^{\circ 000}$

48

cm3

(48.3-71)

(68-109)

(57-167)

(43-54)

median (IQR)

$\begin{array}{lcccc}\text { Ejection } & 56.5 & 44.1^{@ @ ~} & 39.6^{\infty \infty} & 64.0 \\ \text { Fraction } & (51.7-62.9) & (34-53.5) & (31.3-52.7) & (58.8-66.5)\end{array}$

(ECHO) \%

median (IQR)

${ }^{*} p<0.05,{ }^{* *} p<0.005$ compared to population controls;

$@_{p<0.05}$, @ $p<0.005$ compared to Group 1;

${ }^{\infty}<<0.05,{ }^{\infty} p<0.0005$ compared to Group 1] 


\section{TABLE 8.7}

\section{LEFT VENTRICULAR SYSTOLIC DYSFUNCTION \\ - SPEARMAN CORRELATIONS}

GROUP 1 (Normal LV function)

\begin{tabular}{|c|c|c|c|c|c|c|}
\hline & \multicolumn{2}{|c|}{ FIBRINOGEN } & \multicolumn{2}{|c|}{ D- DIMER } & \multicolumn{2}{|c|}{$\begin{array}{l}\text { von Willebrand } \\
\text { factor }\end{array}$} \\
\hline & $\mathbf{r}$ & p & $\mathbf{r}$ & p & $\mathbf{r}$ & p \\
\hline D-dimer & 0.26 & 0.135 & $\ldots$ & & & \\
\hline VWF & 0.28 & 0.118 & 0.009 & 0.956 & $\ldots$ & \\
\hline age & -0.170 & 0.662 & 0.212 & 0.227 & -0.076 & 0.68 \\
\hline $\begin{array}{l}\text { ejection } \\
\text { fraction } \\
\text { (RNVG) }\end{array}$ & -0.36 & 0.187 & -0.436 & 0.101 & -0.26 & 0.618 \\
\hline $\begin{array}{l}\text { body } \\
\text { mass index }\end{array}$ & -0.054 & 0.761 & -0.149 & 0.595 & 0.166 & 0.633 \\
\hline $\begin{array}{l}\text { left atrial } \\
\text { dimension } \\
\text { (long-axis) }\end{array}$ & 0.26 & 0.14 & 0.126 & 0.51 & 0.33 & 0.068 \\
\hline $\begin{array}{l}\text { left atrial } \\
\text { volume }\end{array}$ & 0.02 & 0.901 & 0.142 & 0.555 & 0.342 & 0.062 \\
\hline $\begin{array}{l}\text { LV end- } \\
\text { diastolic } \\
\text { dimension }\end{array}$ & 0.106 & 0.599 & -0.157 & 0.57 & 0.43 & $0.026^{*}$ \\
\hline $\begin{array}{l}\text { LV fractional } \\
\text { shortening } \\
\text { LVEF (echo) }\end{array}$ & -0.425 & $0.023^{*}$ & -0.182 & 0.644 & -0.009 & 0.963 \\
\hline $\begin{array}{l}\text { LV EF } \\
\text { (echo) }\end{array}$ & -0.42 & $0.024^{*}$ & -0.27 & 0.166 & 0.0195 & 0.92 \\
\hline
\end{tabular}


GROUP 2 (LV dysfunction)

\begin{tabular}{|c|c|c|c|c|c|c|}
\hline & \multicolumn{2}{|c|}{ FIBRINOGEN } & \multicolumn{2}{|c|}{ D- DIMER } & \multicolumn{2}{|c|}{ von Willebrand } \\
\hline & $\mathbf{r}$ & $\mathbf{p}$ & $\mathbf{r}$ & $\mathbf{p}$ & $\mathbf{r}$ & $\mathbf{p}$ \\
\hline D-dimer & 0.034 & 0.85 & $\cdots$ & & & \\
\hline vWF & -0.02 & 0.904 & -0.087 & 0.651 & $\ldots$ & \\
\hline age & 0.114 & 0.549 & 0.57 & $0.001^{*}$ & -0.09 & 0.65 \\
\hline $\begin{array}{l}\text { ejection } \\
\text { fraction } \\
\text { (RNVG) }\end{array}$ & 0.000 & 0.99 & -0.04 & 0.831 & -0.144 & 0.512 \\
\hline $\begin{array}{l}\text { body } \\
\text { mass index }\end{array}$ & 0.094 & 0.62 & -0.05 & 0.773 & -0.149 & 0.561 \\
\hline $\begin{array}{l}\text { left atrial } \\
\text { dimension } \\
\text { (long-axis) }\end{array}$ & 0.133 & 0.52 & 0.472 & $0.007^{\star}$ & -0.444 & $0.013^{*}$ \\
\hline $\begin{array}{l}\text { left atrial } \\
\text { volume }\end{array}$ & 0.083 & 0.66 & 0.35 & 0.051 & -0.37 & $0.04^{*}$ \\
\hline $\begin{array}{l}\text { LV end- } \\
\text { diastolic } \\
\text { dimension }\end{array}$ & -0.031 & 0.882 & 0.4176 & $0.04^{\star}$ & 0.03 & 0.887 \\
\hline $\begin{array}{l}\text { LV fractional } \\
\text { shortening } \\
\text { LVEF (echo) }\end{array}$ & 0.375 & 0.068 & -0.34 & 0.101 & -0.1 & 0.658 \\
\hline $\begin{array}{l}\text { LV EF } \\
\text { (echo) }\end{array}$ & 0.37 & 0.074 & -0.35 & 0.087 & -0.111 & 0.619 \\
\hline
\end{tabular}


Group 3a (LV aneurysms, no warfarin)

\begin{tabular}{|c|c|c|c|c|c|c|}
\hline & \multicolumn{2}{|c|}{ FIBRINOGEN } & \multicolumn{2}{|c|}{ D- DIMER } & \multicolumn{2}{|c|}{$\begin{array}{l}\text { von Willebrand } \\
\text { factor }\end{array}$} \\
\hline & $r$ & p & $\mathbf{r}$ & p & $r$ & p \\
\hline D-dimer & 0.121 & 0.55 & $\ldots$ & & & \\
\hline VWF & 0.224 & 0.251 & -0.09 & 0.65 & ," & \\
\hline age & 0.02 & 0.915 & 0.361 & 0.056 & 0.015 & 0.94 \\
\hline $\begin{array}{l}\text { ejection } \\
\text { fraction } \\
\text { (RNVG) }\end{array}$ & 0.276 & 0.161 & -0.08 & 0.694 & 0.0095 & 0.962 \\
\hline $\begin{array}{l}\text { body } \\
\text { mass index }\end{array}$ & -0.186 & 0.655 & -0.192 & 0.671 & 0.086 & 0.662 \\
\hline $\begin{array}{l}\text { left atrial } \\
\text { dimension } \\
\text { (long-axis) }\end{array}$ & -0.07 & 0.72 & 0.076 & 0.704 & -0.236 & 0.224 \\
\hline $\begin{array}{l}\text { left atrial } \\
\text { volume }\end{array}$ & 0.043 & 0.821 & 0.307 & 0.109 & -0.11 & 0.584 \\
\hline $\begin{array}{l}\text { LV end- } \\
\text { diastolic } \\
\text { dimension }\end{array}$ & 0.09 & 0.703 & -0.198 & 0.621 & -0.365 & 0.091 \\
\hline $\begin{array}{l}\text { LV fractional } \\
\text { shortening } \\
\text { LVEF (echo) }\end{array}$ & 0.095 & 0.671 & 0.046 & 0.83 & 0.237 & 0.276 \\
\hline $\begin{array}{l}\text { LV EF } \\
\text { (echo) }\end{array}$ & 0.09 & 0.685 & 0.027 & 0.899 & 0.226 & 0.299 \\
\hline
\end{tabular}




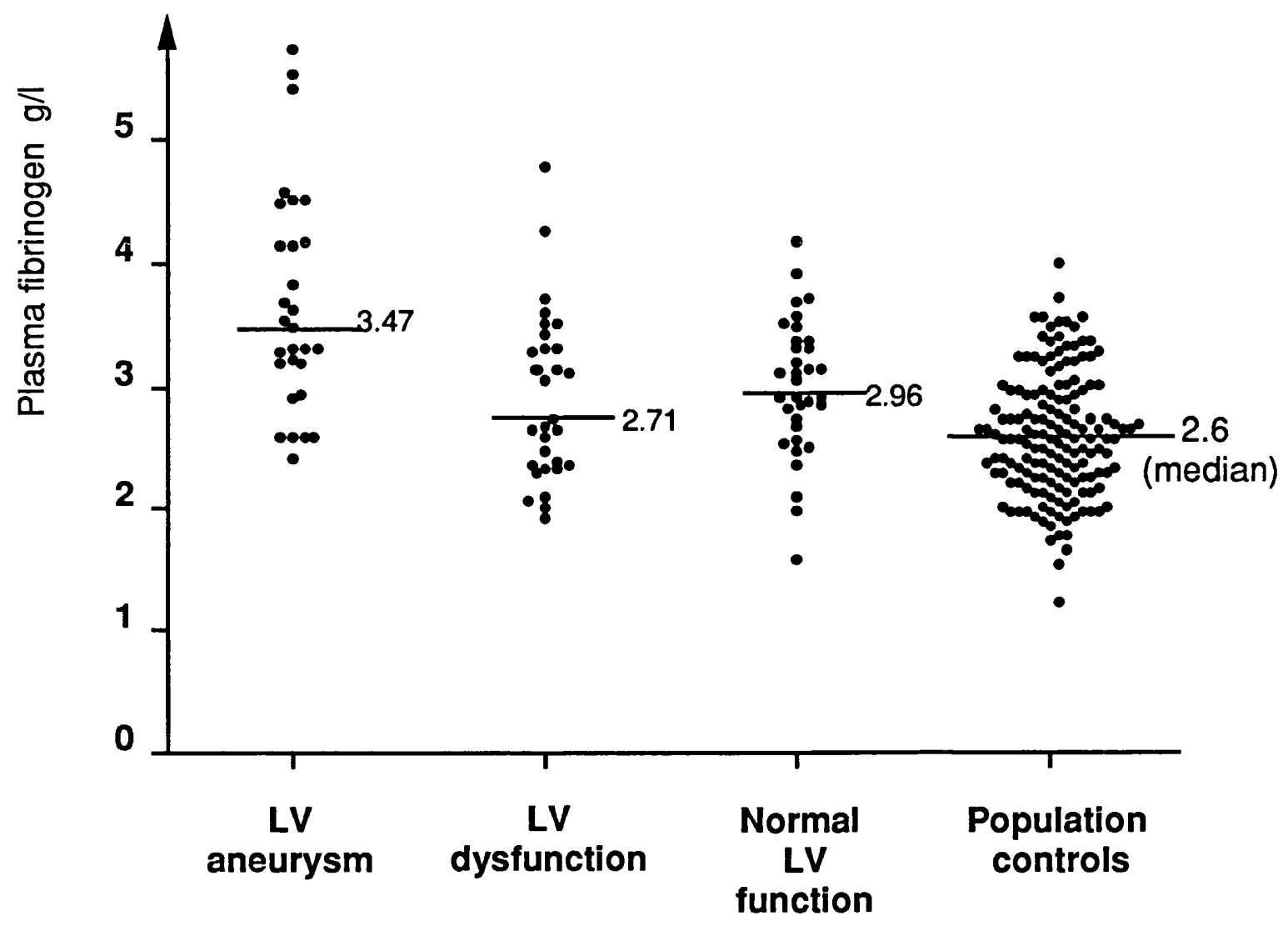

FIGURE 8.1

PLASMA FIBRINOGEN AND LEFT VENTRICULAR FUNCTION 


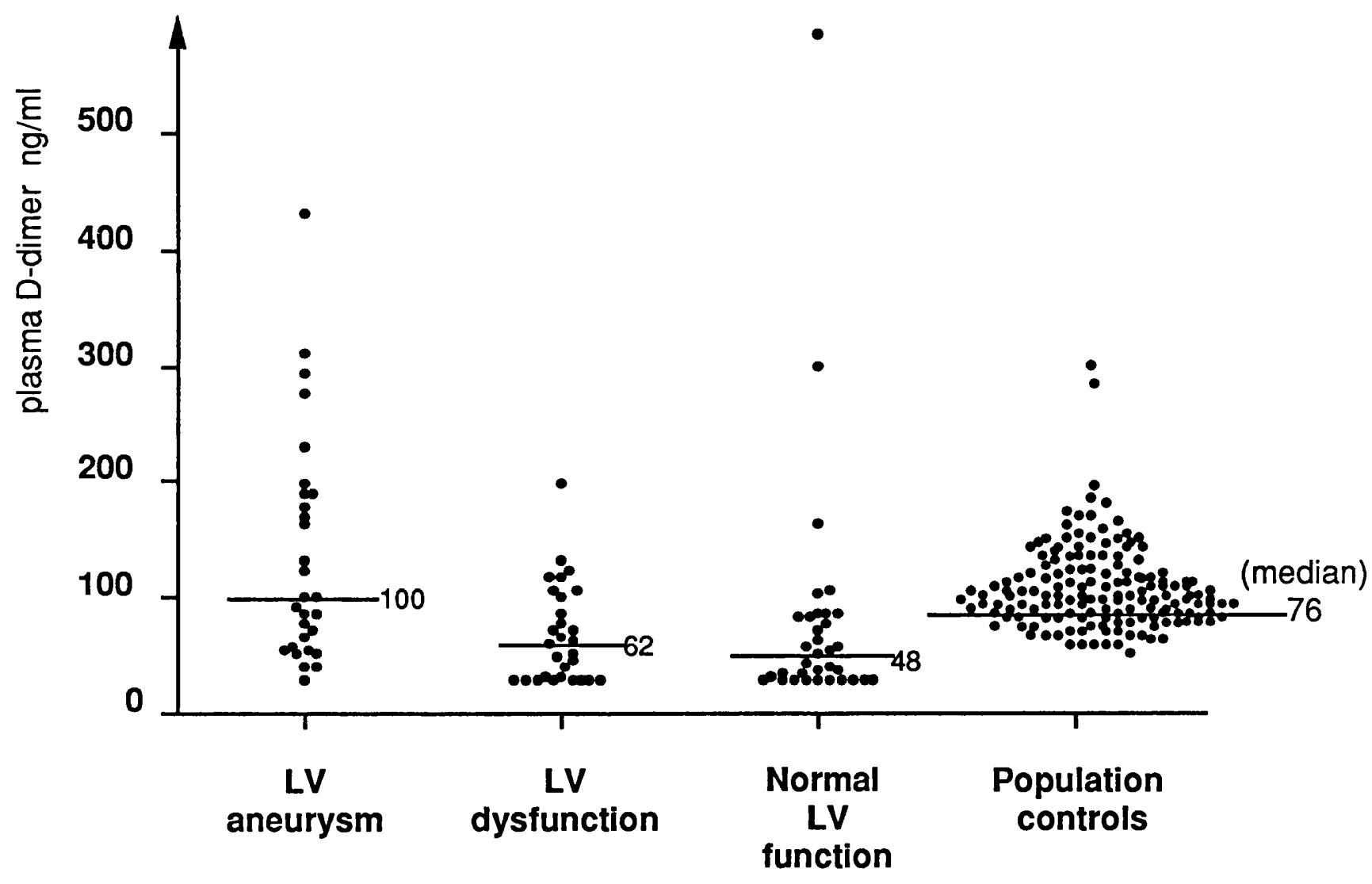

FIGURE 8.2

PLASMA D-DIMER AND LEFT

VENTRICULAR FUNCTION 


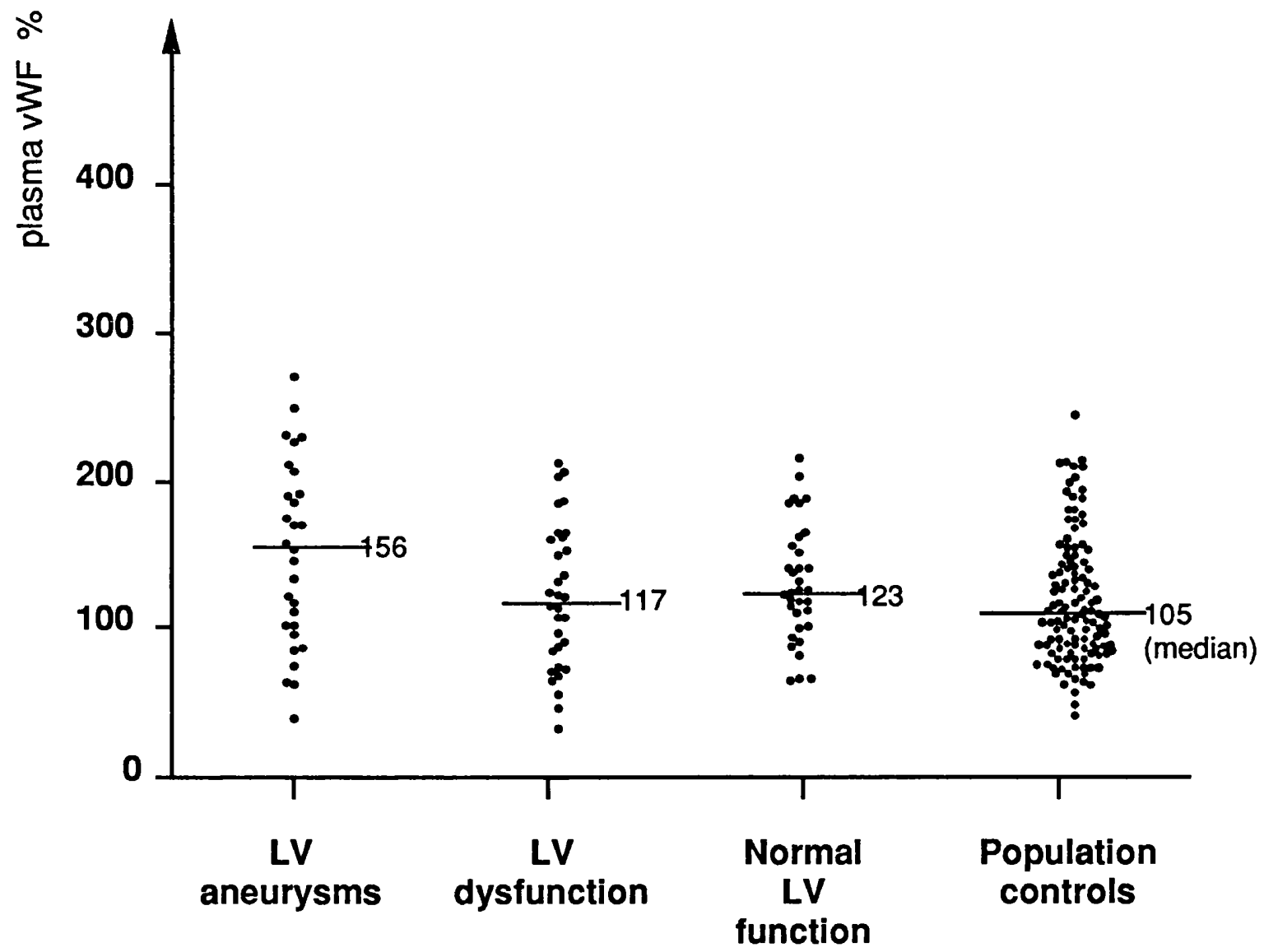

FIGURE 8.3

von WILLEBRAND FACTOR AND LV FUNCTION 
CHAPTER 9

\author{
THROMBOGENESIS \\ IN \\ LEFT VENTRICULAR \\ DIASTOLIC DYSFUNCTION
}




\subsection{IS DIASTOLIC DYSFUNCTION IMPORTANT ?}

\section{Introduction}

Heart failure has been traditionally regarded as due to abnormalities in the systolic or contractile function of the heart. However, up to $30-40 \%$ of patients with congestive heart failure have normal systolic function (Dougherty et al 1984, Johnson 1991, Soufer et al 1985). Instead, the majority of this patient group have abnormal diastolic function as the prime underlying disorder. The clinical presentation and outcome of these patients with congestive heart failure, secondary to diastolic dysfunction, can in fact be identical to those with systolic dysfunction making differentiation between the two conditions very difficult (Setaro et al 1992). There is therefore debate whether or not diastolic dysfunction is clinically important and worthwhile distinguishing from systolic dysfunction in patients with heart failure.

\section{Pathophysiology}

The normal cardiac cycle consists of two components: systole (contraction; ventricular emptying) and diastole (dilatation; ventricular filling). Diastole can be divided into 4 phases: isovolumetric relaxation, early filling, diastasis, and atrial systole. The amount of left ventricular filling occurring during each of these phases depends upon several factors: myocardial relaxation, the passive characteristics of the ventricle and characteristics of the left atrium, pulmonary veins, mitral valve and heart rate (Little and Downes 1990). Diastolic left ventricular function appears, however, to be primarily modulated by diastolic left ventricular pressure and other properties of the left ventricular myocardium (Figure 9.1). When diastolic function is normal, the net effect is left ventricular filling sufficient to produce an adequate cardiac output, while the mean pulmonary venous pressure is maintained below $12 \mathrm{~mm} \mathrm{Hg}$ (Little and Downes 1990). If the latter exceeds $12-15 \mathrm{~mm} \mathrm{Hg}$, as in cardiac failure, pulmonary congestion occurs.

The main causes of diastolic dysfunction are left ventricular hypertrophy, ischaemic heart disease and restrictive cardiomyopathy (Bonaduce et al 1989, Grossman 1990, Setaro et al 1992, Shepherd et al 1989). Other causes include valvular disease, 
chronic renal failure, diabetes and restrictive or constrictive states (Grossman 1990). In all these conditions, impaired myocardial relaxation, ventricular hypertrophy and fibrosis occur. This results in stiff, poorly relaxing, ventricles that fill adequately only under high atrial pressure. This problem is exacerbated during exercise when the diastolic filling time is reduced. The net result may therefore be pulmonary congestion despite normal systolic pumping function, and possible subendocardial ischaemia, development of compensatory myocardial hypertrophy and consequent detrimental remodelling of the heart (Harizi et al 1988, Pouleur et al 1989). The main conditions associated with diastolic dysfunction (as discussed above) are also associated with thromboembolism. Hypertrophic cardiomyopathy, for example, constitutes an important thromboembolic risk, and poor ventricular compliance is present in such patients (Nishide et al 1983, Petrin and Tavel 1979).

Ischaemic heart disease is probably the most common condition associated with impaired diastolic function. Importantly, diastolic function is more susceptible to ischaemia than systolic function and can take longer to recover. Silent myocardial ischaemia in asymptomatic patients with coronary artery disease may result in significant diastolic dysfunction (Mahmarian and Pratt 1990) (Figure 9.2). Furthermore, impaired ventricular distensibility may adversely affect the Frank-Starling mechanism, thus impairing systolic pump function and cardiac output adaptation during exercise (Pouleur et al 1989). Systolic and diastolic dysfunction may (and often do) co-exist, for example, in an infarcted segment where fibrosis and scar formation may result in both significant wall motion abnormalities and decreased diastolic distensibility of the left ventricle (Bonow 1990) (Figure 9.2). Such 'regional' fibrosis results in increased stiffness of the ventricular chamber; and the presence of akinesia or dyskinesia (as in aneurysm formation) creates regional systolic dyssynchrony, which adds further to impaired ventricular relaxation (Bonow 1990). In short, when diastolic dysfunction is present, there is often a mechanical problem: the ventricular chambers can no longer accept, at normal pressure, enough blood to maintain normal stroke volume.

There is some evidence that significant biochemical alterations within the myocyte can also cause diastolic dysfunction, although the associated mechanisms may be related to ischaemia or hypoxia. Examples of such biochemical changes include increased 
intracellular calcium and decreased adenosine triphosphate and creatine phosphate (Grossman 1990).

The measurement of diastolic dysfunction is made difficult as it is associated with several clinical parameters, such as cardiac rhythm, heart rate, end-diastolic left ventricular volume, systolic left ventricular performance and age (Marcus and Dellsperger 1991, Stewart et al 1992). A method of adjusting for the influence of age and heart rate on diastolic function however has been described (Stewart et al 1992). Atrial systolic function is also an important consideration. In the young healthy individual, under normal circumstances, left atrial systole contributes less than $25 \%$ of left ventricular stroke volume. However, in the presence of diastolic dysfunction, tachycardia or advancing age, the atrial contribution may become much more significant, contributing up to $50 \%$ of left ventricular stroke volume (Little and Dowes 1990, Marcus and Dellsperger 1991, Takemoto et al 1992). Exercise has an additional influence on this (which is independent of heart rate) and left ventricular diastolic dysfunction associated with 'normal' aging is less pronounced in those persons who are exercise-trained (Takemoto et al 1992).

In summary, impaired early ventricular filling and a greater dependence on atrial systole for ventricular filling would typify most cases of diastolic dysfunction (Harizi et al 1988, Labovitz and Pearson 1987, Little and Dowes 1990, Stauffer and Gaasch 1990).

\section{Methods of diagnosis}

The cardinal symptom in patients with diastolic dysfunction is dyspnoea. The acute clinical presentation may frequently be identical to that of systolic dysfunction, and the clinical differentiation between the two is often difficult, if not impossible, without further investigation (such as echocardiography). The presence of diastolic dysfunction is suggested by pulmonary congestion in a patient with a normal heart size on the chest X-ray. However, cardiomegaly and signs of biventricular failure (elevated jugular venous pressure, basal crepitations and oedema) are also frequently present (Dougherty et al 1984, Soufer et al 1985). In sinus rhythm, a fourth heart sound is invariably present, and although a left ventricular third heart sound usually indicates systolic dysfunction, it may also be heard in 
isolated diastolic dysfunction with an extremely elevated left atrial pressure (Dougherty et al 1984, Soufer et al 1985).

There is unfortunately no single method of investigation that may be used to characterise left ventricular diastolic dysfunction, but the two most useful techniques are echocardiography and radionuclide ventriculography (Marcus and Dellsperger 1991).

Echocardiography is still the most common and most frequently used noninvasive method. Doppler echocardiography has been favourably compared with both angiographic (Rokey et al 1985) and radionuclide techniques (Friedman et al 1986, Spirito et al 1986) in the assessment of diastolic dysfunction. One of the most frequently used methods is to measure the transmitral flow by Doppler echocardiography (Thomas and Weyman 1991) (Figure 9.3). Normal transmitral flow can be divided into early and late atrial filling phases, the latter resulting from atrial contraction. The typical change seen, for example, in severe heart failure, is a rise in early peak flow velocity and an increased ratio between early and atrial peak flow velocity when high pressure is present in the left atrium. These patterns are however reversed with reduced left ventricular compliance and impaired diastolic function (for example, in hypertension, cardiomyopathy and coronary artery disease) (Cheng 1990, St Goar et al 1991, Thomas and Weyman 1991). Other empirical indices of diastolic dysfunction have been derived from the mitral flow pattern, such as the integrated velocities of early rapid filling and atrial contraction, their ratios, and the acceleration and deceleration times of the early filling wave, but these indices are not well established (Thomas and Weyman 1991). Another measurement is the atrial ejection force, which is a measure of atrial systolic function, that allows an assessment of the atrial contribution to diastolic performance (Manning et al 1993). Atrial ejection force is dependent upon atrial preload, atrial afterload and ventricular stiffness, and as its calculation takes into account the acceleration of blood (as ejected by the atria) and the mass of blood ejected through the mitral orifice, it is considered as a more physiological index of atrial systolic performance (Manning et al 1993).

The major advantage of Doppler echocardiography is its ease in providing a noninvasive evaluation of diastolic function on a beat-by-beat basis with a high success rate (Rokey et al 1985). Doppler indices are, however, only indirect measures of diastolic function, 
and provide no direct assessment of either ventricular relaxation or compliance. The use of Doppler measurements of mitral inflow velocities has however been validated in comparison to angiographic parameters of ventricular filling (Labovitz and Pearson 1987, Rokey et al 1985). The relationship is good, with a strong favourable correlation between the ratio of peak early to atrial velocities, the ratio of early to atrial flow velocity integrals and left ventricular diastolic pressure (Appleton et al 1988b, Labovitz and Pearson 1987).

Another Doppler pattern of diastolic dysfunction different from the classic E/A velocity reversal has been described. This is characterised by a short isovolumetric relaxation time, a tall narrow $E$ wave with high peak velocity and steep deceleration, and a small narrow $\mathbf{A}$ wave. This Doppler pattern is observed in situations with very low ventricular compliance such as constrictive pericarditis (Hatle et al 1989), restrictive cardiomyopathy (Appleton et al 1988a, St Goar et al 1991) and acute severe aortic insufficiency (Oh et al 1989). It is thought to be due to abnormalities in the early, energy dependent diastolic filling period (Doppler E velocity), whereas late diastolic dysfunction (the Doppler A velocity) is more affected by the passive distensibility of the chamber ("compliance") (Devereux 1989). Therefore, increasing chamber stiffness may result in an enhanced peak early filling velocity and volume, with decreased filling during atrial systole (Stoddard et al 1989). Impaired relaxation, however, correlates with a decreased peak early filling velocity and increased atrial contribution to filling (Stoddard et al 1989).

Echocardiography does however have its limitations in the assessment of diastolic function. One is the visual measurement of the mitral annulus and the assumption of a constant circular geometry to the mitral valve annulus throughout diastole. Secondly, measurement of Doppler mitral valve velocities can be inaccurate in the presence of associated valvular heart disease and are of limited value during exercise as early and late diastolic profiles tend to merge at heart rates of 100 or more beats per minute.

In summary, there are two echocardiographic patterns of diastolic dysfunction, the first reflecting abnomal relaxation and characterised by a high peak atrial velocity and diminished early filling velocity (and the ratio of these velocities, E/A vel $<1$ ); the 
second reflecting a restrictive or constrictive pattern, with a high early filling velocity and a small narrow A wave (with their ratio, E/A vel usually >2). This is illustrated in Figure 9.4.

In multigated radionuclide ventriculography, a reduction in peak left ventricular filling rate and prolongation of time to peak filling indicate impaired early ventricular filling, which is present in diastolic dysfunction. Radionuclide studies remain the 'gold standard" method although they have significant limitations. It is important to acquire a high quality study with good target to background ratio. Thus subjects (for example, diabetics) who bind the radionuclide label poorly to their erythrocytes and those with frequent ventricular ectopics yield poor information. The images should be as 'noise-free' as possible and the time activity curve generated with respect to end-diastolic volume subtraction and normalisation to ventricular rate for standardisation. Analysis of higher harmonics, other than the fundamental total body count can be useful but increases the complexity.

One other technique for assessing diastolic function involves cardiac catheterisation, but this has essentially gone out of favour. Using this technique, patients with left ventricular dysfunction can be characterised by the following features: (i) an elevated left ventricular end-diastolic pressure $\geq 15 \mathrm{~mm} \mathrm{Hg}$; (ii) normal left ventricular end-diastolic $(<90$ $\mathrm{mL} / \mathrm{m}^{2}$ ) and end- systolic ( $<35 \mathrm{~mL} / \mathrm{m}^{2}$ ) volumes; (iii) normal left ventricular ejection fraction ( $\geq 0.50$ ); and (iv) the absence of valve disease (Brogan et al 1992).

In summary, there is no absolute 'gold standard' for assessing diastolic performance. It is difficult to state which method (or index) is the most reliable or accurate measure of diastolic function. However, both echocardiography and radionuclide methods are minimally invasive, relatively simple, with capability for serial study; and are therefore most useful in clinical practice.

Are the differences between systolic and diastolic dysfunction prognostically important for cardiovascular events and thromboembolic risk?

Epidemiological studies have established the contributory role of heart failure to thromboembolic risk but the individual contributions of systolic and diastolic dysfunction have not been established. For example, ischaemic heart disease, hypertension and 
cardiomyopathy are common causes of diastolic dysfunction (Setaro et al 1992) and these conditions are also associated with a significant thromboembolic risk. The presence of hypertensive heart disease is also additive to the presence of atrial fibrillation as a risk for stroke (Stroke Prevention in Atrial Fibrillation Investigators 1992a) and echocardiographic abnormalities of diastolic filling are often seen in patients with hypertension and left ventricular hypertrophy (Bonaduce et al 1989, Shepherd et al 1989). In addition, a common aetiological factor for both atrial fibrillation and diastolic dysfunction is ischaemic heart disease, which was present in $47 \%$ of acute admissions with atrial fibrillation to my hospital (see chapter 2).

Clinical evidence for the deleterious effects of diastolic dysfunction is provided in a follow-up study of $\mathbf{5 2}$ patients initially hospitalised with congestive heart failure with intact left ventricular systolic function (Ejection Fraction $>45 \%$ ). Combined cardiovascular mortality and morbidity at 7 years was $75 \%$ whilst noncardiovascular mortality was $10 \%$ (Setaro et al 1992). Interestingly, cardiovascular mortality in these patients (46\%) included refractory congestive heart failure and pulmonary oedema, myocardial infarction and stroke (Setaro et al 1992). These data would suggest that diastolic dysfunction, by virtue of its effects in promoting congestive cardiac failure, would predispose patients to thrombotic and embolic risks, and an excess was noted in this study although not analysed in detail.

Diastolic dysfunction may, however, have a better overall long-term prognosis than systolic dysfunction. For example, both the CASS Registry and the V-HeFT study demonstrated a better prognosis for congestive heart failure patients with normal systolic function when compared to those with systolic dysfunction (Cohn and Johnson 1990, Judge et al 1991). Brogan et al (1992) also showed a low cardiac mortality (14\%) but a substantial morbidity (45\%) in such patients. However, an important consideration is the link between diastolic dysfunction and silent myocardial ischaemia (Mahmarian and Pratt 1990). Seemingly healthy asymptomatic patients and patients with stable ischaemic heart disease, unstable angina, or recent myocardial infarction are all at higher risk of subsequent cardiovascular morbidity (and perhaps mortality) if silent ischaemia is present, and underlying diastolic dysfunction may in part contribute to this risk (Mahmarian and Pratt 1990). Patients with 
congestive heart failure and intact systolic function therefore do not have a favourable outcome (Setaro et al 1992, Warren and Grossman 1991).

\section{Management options}

The treatment of patients with diastolic dysfunction is unfortunately difficult. This is pertinent as diastolic dysfunction is common in ischaemic heart disease and abnormalities of diastolic rather than systolic performance may be important determinants of the clinical status and exercise intolerance of patients with heart failure (Packer 1990). Drugs that lower ventricular filling pressures are more likely therefore to enhance exercise capacity than drugs that primarily increase cardiac output and left ventricular ejection fraction indices (Packer 1990).

Along these lines, treatment with verapamil appears to have therapeutic benefit (Shepherd et al 1989). Other useful therapies to be considered include xamoterol (a partial beta agonist), beta agonists (Pouleur et al 1989, Rousseau et al 1989) and negatively inotropic agents such as disopyramide. Beta blockers, however, can also have variable therapeutic effects in diastolic dysfunction, due to the associated changes in heart rate and afterload (Shepherd et al 1989). In contrast, therapy with cardiac glycosides, such as digoxin, appears to be of no benefit and theoretically these drugs could be detrimental (Pouleur et al 1989). Diuretics and nitrates may also be detrimental in patients with diastolic dysfunction by decreasing venous return and filling pressures, resulting in decreased cardiac output and hypotension (Pouleur et al 1989, Shepherd et al 1989). The position is less clear for angiotensin-converting enzyme inhibitors (Pouleur et al 1989), but some benefits on diastolic dysfunction post-myocardial infarction have been shown (Gøtzsche et al 1992). 


\subsection{STUDIES OF FIBRINOGEN, VON WILLEBRAND FACTOR AND D-DIMER IN DIASTOLIC DYSFUNCTION}

Introduction

Current clinical and epidemiological data on the contributory role of heart failure to thromboembolic risk does not differentiate between systolic and diastolic dysfunction, although coronary artery disease, hypertension and cardiomyopathy (all commonly associated with thromboembolic risk) are the most frequent causes of diastolic dysfunction. Increased ventricular 'stiffness' and poor myocardial compliance may cause sufficiently impaired ventricular filling to result in abnormal blood flow characteristics adequate to promote thrombogenesis. Indeed, the presence of a large ventricular wall motion abnormality (in association with a reduced ejection fraction) may result in an increase in left ventricular end-diastolic volume or pressure and diminished overall left ventricular compliance (Cheng 1990).

Doppler echocardiography represents an excellent technique capable of simply and noninvasively measuring the timing, magnitude and patterns of blood flow in cardiac chambers during both diastole and systole. The aim of this echocardiographic study was to determine the prevalence of diastolic dysfunction in patients with ischaemic heart disease, and whether or not the presence of left ventricular systolic dysfunction (and left ventricular aneurysm formation) alters this prevalence. Secondly, I wished to assess whether or not diastolic dysfunction contributes to the prothrombotic state (as measured by indices of thrombogenesis such as plasma fibrinogen, von Willebrand factor and fibrin D-dimer) of patients with left ventricular dysfunction.

\section{Materials and methods}

The design was a prospective case controlled study of patients with underlying coronary artery disease, all of whom were in sinus rhythm. A diagnosis of coronary artery disease was established in all patients as a consequence of either coronary 
angiography or a clinical history of myocardial infarction, or angina with demonstrable ischaemia on exercise stress testing.

Left ventricular systolic function (and the presence of an aneurysm) was assessed by at least two of three methods described in chapter 8 . Briefly these methods are review of the dynamic single-plane left ventriculogram (performed at cardiac catheterisation), echocardiography and $750 \mathrm{MBq} 99 \mathrm{~m}$ Technetium isotope radionuclide ventriculogram (RNVG). Unfortunately our computer in Stobhill Hospital was not sophisticated enough to measure indices of diastolic dysfunction using radionuclide venticulography.

Echocardiography was performed using a Hewlett Packard Sonos 500 machine (Hewlett-Packard, Arondale, Pa, USA) using a standard $2.5 \mathrm{MHz}$ transducer for pulsed wave Doppler studies. All subjects were studied in the lateral decubitus position. Normal Doppler indices were derived from 14 healthy volunteers who served as controls. Patients with significant mitral or aortic regurgitation and those with hypertension or left ventricular hypertrophy (left ventricular posterior wall in diastole $>1.1 \mathrm{~cm}$ ) were excluded. The left ventricular mass index was not used as an exclusion criteria as standard formulae for its calculation are only valid in left ventricles without wall motion abnormalities or dyskinetic segments. In addition, patients with pericardial disease, cardiomyopathy, moderate / severe mitral (with an abnormal MV $\mathrm{Pt}_{1 / 2}$ ) or aortic valve disease, a heart rate $<50 / \mathrm{min}$ or $>100 / \mathrm{min}$ or rhythm other than sinus rhythm were excluded. Only subjects with good quality Doppler echocardiographic recordings were included for analysis.

Doppler examination was performed using colour-flow, pulsed and continuous wave modes. Forward transmitral flow was determined in all patients. Peak mitral flow velocity was initially identified by continuous wave Doppler and then recorded using the pulsed wave mode and a $3 \mathrm{~mm}$ gate from a position within the left ventricle at the level of the mitral annulus. Using the standard video facility of the ultrasound machine, off line analytical methodology was used to quantify measurements. Mean values from at least five cardiac cycles were used in the analyses. The peak velocities of the early (E) wave and the atrial $(A)$ wave were recorded and their ratio $\left(E_{V \max } / A_{V \max }\right)$ derived. The velocity time integral (or area under the velocity curve) for the early (E) and atrial (A) waves were also measured by tracing the contour of the darkest portion of the 
curve (Figure 9.3). In cases in which the early filling curve overlapped that of the atrial filling curve, a line dropped from the point representing the nadir of the two curves was used to separate the two integrals.

The following parameters of diastolic function were also calculated by means of the mitral inflow velocities: (i) peak filling rate $\left(\mathrm{cm}^{3}\right.$ per second $)=$ peak early diastolic velocity $\left(E_{V \max }\right) \times$ cross-sectional area of the mitral annulus; (ii) normalised peak filling rate (in seconds ${ }^{-1}$ ) = peak filling rate divided by the left ventricular end-diastolic volume; and (iii) atrial filling rate = peak atrial velocity $\left(A V_{\max }\right) \times$ cross-sectional area of the mitral annulus. As a measure of the balance between early and late diastolic filling, the following ratios were also calculated: (i) the ratio of the integral of the early diastolic filling period to that of the atrial filling period ( $\left.E_{i n t} / A_{i n t}\right)$; and (ii) the ratio of the peak early to peak atrial velocity $\left(E_{V \max } / A_{V \max }\right)$. Finally, as an index of atrial systolic function, the atrial ejection force was calculated as $0.5 \times \rho \times$ mitral orifice area $\times$ (peak $A$ velocity, $\left.A_{V \max }\right)^{2}$ [where density of blood, $p=1.06 \mathrm{~g} / \mathrm{cm}^{3}$ ] (Manning et al 1993).

M-mode recordings of the left ventricular cavity were made at the level just below the mitral leaflets to derive the left ventricular fractional shortening fraction and left ventricular enddiastolic volume. Fractional shortening was calculated as (LV end-diastolic dimension - LV endsystolic dimension) / LV end-diastolic dimension. Left ventricular end-diastolic volume was calculated as the cube of the left ventricular end diastolic diameter. To calculate the mitral annulus area, measurements were performed from the apical four-chamber and parasternal long-axis views during early diastole. Measurements were taken from the inner edge of the lateral bright corner of the annulus to the inner edge of the medial corner just below the insertion of the mitral leaflets, and the mitral annulus diameter averaged from the two views. The cross-sectional area of the annulus was derived as (3.14) $\times$ (mitral annular diameter / 2) 2 .

To allow validation of the Doppler measurements, and to assess reproducibility, interobserver and intraobserver variability, a sample of 20 patients selected at random were studied twice on different occasions by the same observer (myself) and another 20 subjects on different occasions by two observers (myself and one other observer). Reproducibility for Doppler echocardiographic measurements was expressed as mean difference $\pm 2 s$.d (Bland and Altman 1986). A one-sample t-test was performed to determine whether the mean difference was 
significantly different from zero. Methods of blood sampling and assays for plasma fibrinogen (CLAUSS), D-dimer (ELISA) and von Willebrand factor (ELISA) are as previously described in chapter 3 and 8 ).

Values are quoted as median \pm IQR (interquartile range). For non-parametric comparisons, the Kruskal Wallis test and Mann-Whitney $U$ test were used to compare medians. A $\mathrm{X}^{2}$ test was used to assess differences in sex, smokers, and the prevalence of diastolic dysfunction between groups. Correlations between clinical variables or plasma factors were performed using Spearman's Rank correlation method. Analysis of the influence of systolic and diastolic dysfunction and their interaction was performed using ANOVA for unbalanced data (General Linear Model). A probability value of $<0.05$ was considered significant. All statistical calculations were performed on a microcomputer using a commercially available statistical package (MINITAB v8, Minitab INC, PA,USA)

Results

A total of 106 patients ( 81 male, 25 female; mean age 57.5 years, s.e.m. 0.87 ) with ischaemic heart disease were studied. Of these 5 were excluded as Doppler echocardiography was technically inadequate for further analysis. The remaining 101 patients were sub-divided into 3 groups on the basis of ventricular systolic function: 40 patients with normal left ventricular function (Group 1), 31 patients with impaired systolic function (Group 2, left ventricular systolic dysfunction); and 30 patients with left ventricular systolic dysfunction and aneurysm formation (Group 3, left ventricular aneurysm). They were compared to Doppler echocardiographic parameters of diastolic function in 14 healthy controls ( 8 male, 6 female; mean age 53.2 years, s.e.m. 2.2) of similar age in sinus rhythm (Table 9.1).

Whole group There were no significant differences for age (oneway ANOVA $F=1.12$, $p=0.35)$ and sex distribution $\left(X^{2}=6.5\right.$, d.f. $=3 ; p=0.09$ ) between the groups. Body mass index (Kruskal-Wallis test $H=3.91$, d. $f=2 ; p=0.142$ ) and proportion of smokers $\left(X^{2}=1.81, d . f=2\right.$; $\mathrm{p}=0.40$ ) were also similar in the patient groups studied. Echocardiographic findings for the group as a whole $(n=102)$ are summarised in Table 9.1. Patients in Group 1 (Normal systolic 
function) had significant differences in median $A_{V \max }, A_{i n t}$, the $E_{V \max } / A_{V \max }$ and $E_{i n t} / A_{i n t}$ ratios, and atrial ejection force when compared to healthy controls (all $p<0.05$ ). Patients in Group 2 (Left ventricular dysfunction) had significant differences in median normalised peak filling rate when compared to Group $1(p<0.05)$. There was a higher atrial ejection force in Group 3 (Left ventricular aneurysms) when compared to Group $1(p<0.05)$, and a trend (nonsignificant) towards lower median normalised peak filling rates, EVmax / AVmax and higher EVmax,$A_{V \max }$ and atrial filling rates in Group 3 patients.

Diastolic dysfunction was defined as the presence of an $\mathrm{EV}_{\mathrm{V} \max } / \mathrm{A}_{\mathrm{V} \max }$ ratio of $<0.958$. The value of 0.958 was derived from the lower limit of the $95 \%$ confidence interval for the median EVmax / A Vmax ratio of my healthy control group (median 1.055. 95\% c.i. 0.958-1.148). The prevalence of patients with diastolic dysfunction in each group of patients with coronary artery disease is summarised in Table 9.2. Proportions of patients with diastolic dysfunction were similar (approximately $60 \%$ ) in all three groups $\left(X^{2}=0.28\right.$, d.f. $=2 ; p=0.87$ )

Group 1 (Normal systolic function) Patients in Group 1 with diastolic dysfunction (Group 1a, $n=24)$ had a median plasma levels for fibrinogen of $3.02 \mathrm{~g} / \mathrm{I}($ IQR 2.53 - 3.34), plasma $\mathrm{D}-$ dimer of $46 \mathrm{ng} / \mathrm{ml}$ (IQR 30-82) and von Willebrand factor (VWF) of 123\% (IQR 95-160). Levels of plasma fibrinogen, fibrin D-dimer and WWF were not significantly different when compared to the Group 1 patients without diastolic dysfunction (Group 1b, $n=16$ ). In Group 1a, the correlation between plasma fibrinogen and $D$-dimer levels was significant $(r=0.43, p=0.035)$ (Table 9.3). There was, however, no significant correlation between age, plasma fibrinogen, D-dimer or WWF with Doppler indices of diastolic dysfunction, although a trend is noted for the correlation between plasma vWF and $E_{V \max }(r=-0.42, p=0.06)$ and with $E_{\text {int }}(r=$ $-0.42, p=0.06$ ). In Group $1 b$ (that is, normal systolic and diastolic function), there is a significant correlation between age and peak filling rate $(r=0.77, p=0.001)$ and between $D$-dimer levels and atrial filling rate or atrial ejection force $(r=-0.62, p=0.013)$.

Echocardiographic parameters of diastolic dysfunction of patients in Group 1 are summarised in Table 9.2. There were significant differences in peak filling rate, atrial filling rate (both $p<0.05)$ and atrial ejection force $(p=0.004)$; and also $E_{V \max }, A V_{\max }($ both $p<0.005)$; 
Eint, $A$ int (both $p<0.05), E_{V \max } / A_{V \max }$ and $E_{i n t} / A_{\text {int }}$ (both $p<0.0001$ ) in Group 1a when compared to Group $1 \mathrm{~b}$.

Group 2 (left ventricular systolic dysfunction) Patients in Group 2 with diastolic dysfunction (Group 2a, $\mathrm{n}=19$ ) had median plasma levels for fibrinogen of $3.04 \mathrm{~g} / \mathrm{l}$ (IQR 2.34-3.47), plasma D-dimer of $49 \mathrm{ng} / \mathrm{ml}$ (IQR 30-106) and vWF of 110\% (IQR 69-160). In these patients levels of plasma fibrinogen, fibrin D-dimer and VWF were again not significantly different when compared to the Group 2 patients without diastolic dysfunction (Group 2b, $n=13$ ).

Echocardiographic parameters of diastolic dysfunction of patients in Group 2 are summarised in Table 9.2. There were significant differences in peak filling rate, atrial ejection force, EVmax, $A V_{\max }$ (and their ratio) (all $\left.p<0.05\right)$, Eint $(p<0.01)$, Eint and the Eint/Aint ratio (both $p<0.001$ ) in patients with diastolic dysfunction when compared to controls (Table 9.2). In both Group $2 a$ and $2 b$, there was a negative correlation between vWF levels and peak filling and atrial filling rates (all $p<0.05$ ).

Group 3 (Left ventricular aneurysm) Patients in Group 3 with diastolic dysfunction (Group 3a, $n=18$ ) had median plasma levels for fibrinogen of $3.46 \mathrm{~g} / /$ (IQR $2.92-4.25$ ), plasma $D-$ dimer of 96ng/ml (IQR 65-172) and vWF 173\% (IQR 88-194). Levels of plasma fibrinogen, VWF and fibrin D-dimer in Group 3a were not significantly different when compared to the Group 3b ( $n=12)$. There was a positive correlation between vWF and EVmax and $E$ int $(p<0.05)$. In addition, there was also a positive correlation between D-dimer and atrial filling rate, and between D-dimer and atrial ejection force $(p<0.05)$.

Echocardiographic parameters of diastolic dysfunction of patients in Group 3 are summarised in Table 9.2. There were significant differences in all Doppler parameters of diastolic dysfunction when Group 3a patients were compared with Group $3 b$ (all $p<0.05$ ).

Role of aneurysms, systolic and diastolic dysfunction (and their interaction) on thrombogenesis The relative contributions of systolic and diastolic function, the presence of an aneurysm and their interactions on plasma fibrinogen, D-dimer and vWF levels 
are summarised in Table 9.4. Using ANOVA for unbalanced data (GLM or General Linear Model analysis), the presence of an aneurysm or systolic dysfunction was associated with difference in plasma fibrinogen $(F=9.96, d . f=2, p<0.0001)$ and vWF levels $(F=3.32, d . f .=2$, $p=0.041$ ), with no significant effect of diastolic dysfunction (or the interaction between them) on these prothrombotic factors. There was also a trend towards a difference in D-dimer levels in patients with systolic dysfunction and aneurysms but this did not reach significance $(F=2.15, d . f=2, p=0.12)$. There was no effect of diastolic dysfunction (or the interaction with systolic abnormalities) on D-dimer levels.

\section{Discussion}

This study represents a detailed Doppler echocardiographic assessment of left ventricular diastolic dysfunction in patients with coronary artery disease and systolic dysfunction. I have also demonstrated that there were no significant abnormalities in plasma fibrinogen, fibrin D-dimer fragment and von Willebrand factor levels in patients with diastolic dysfunction when compared to those without diastolic dysfunction.

Diastolic dysfunction secondary to coronary artery disease was common (approximately 60\%) in each of my patient groups studied. The impairment of diastolic function was observed both in patients with and without systolic dysfunction, although the degree of diastolic dysfunction appeared to be greater in patients with concomitant systolic dysfunction and ventricular aneurysm formation. This high prevalence is not due to factors such as age or sex, with no significant differences between the groups studied. Neither is it due to an excess of hypertension and significant rheumatic valve disease (which may cause alterations in diastolic function (Shaikh and Lavine 1988, Shepherd et al 1989)), as these patients were specifically excluded from the study. There was a high proportion of patients with prior myocardial infarction in the group with left ventricular aneurysms (as expected) and this may have contributed to the prevalence of diastolic dysfunction in Group 3. Nevertheless, the proportion of patients with myocardial infarction was not similar to the proportion of patients with diastolic dysfunction. 
Whilst I have observed a high prevalence of diastolic dysfunction in this study, my results are consistent with previous observations of diastolic dysfunction in a similar patient population using radionuclide techniques (Bonow et al 1981, Harizi et al 1988). For example, in patients with coronary artery disease and a normal ejection fraction, abnormalities of diastolic function have been reported in $\mathbf{5 2}$ to $86 \%$ of patients; whilst in patients with systolic dysfunction, abnormalities of diastolic filling have been noted in 69 to $100 \%$ of patients (Harizi et al 1988). My study therefore confirms previous observations that diastolic abnormalities are common in patients with ischaemic heart disease and may appear even before systolic abnormalities are evident (Harizi et al 1988). These observations suggest that it may be useful to investigate all patients presenting with congestive cardiac failure to establish the relative contributions of systolic and diastolic dysfunction, in order to optimise the patient's therapy and thereby reduce their morbidity as much as possible. For example, heart failure post-myocardial infarction can result from both systolic and diastolic dysfunction, and therefore detailed Doppler echocardiography in the early post-infarction period can be predictive of subsequent heart failure and morbidity. In one early ( $<36$ hours) post-infarction study, the following predictors of subsequent heart failure were identified: age, anterior infarction, early diastolic peak filling velocity $(E$ Vmax), peak atrial filling velocity (A Vmax), the ratio of these velocities ( $E$ Vmax / $A$ Vmax) and the presence of wall motion abnormalities (Finkelhor et al 1991). There is therefore a view that diastolic heart failure may correlate better with prognosis for symptoms and survival than traditional indices of systolic function (Packer 1990, Warren and Grossman 1991). The combined assessment of both systolic and diastolic function would therefore be very important in the management of such patients.

However, the use of Doppler echocardiography in the assessment of diastolic function has its limitations and caveats. Most studies measure mitral flow velocities ( $E_{V \max }$ and $A_{V \max }$ ) and their ratio, and peak filling rates which have been relatively well validated (Harizi et al 1988, Rokey et al 1985). However the mitral annulus does change in area over the cardiac cycle, resulting in changes in the mitral flow velocity profile (Nishimura et al 1989). This change in mitral flow varies from being directed centrally into the left ventricle in early diastole, to posterolaterally during end-diastole (Nishimura et al 1989). Pulsed Doppler 
recording of the flow velocity through the mitral valve annulus may therefore not accurately reflect instantaneous volumetric flow (Nishimura et al 1989). The highest velocities across the mitral valve are usually recorded with the sample volume at or between the tips of the mitral valve leaflets as they open during diastole, and any recording away from this position may significantly alter the $E$ and $A$ velocity profiles (Nishimura et al 1989). An altered mitral flow velocity curve also occurs with the use of continuous wave Doppler (which sums all the velocities along the Doppler beam); continuous wave Doppler should not therefore be used in assessment of diastolic function. In addition, in the presence of left-sided valvular abnormalities, increased heart rate, multiple ectopics or poor ventricular compliance, mitral flow velocities can significantly change with alterations in ventricular filling (Marcus and Dellsperger 1991).

A further possible limitation in my study is the comparison of Doppler indices with that derived from 14 healthy subjects. Some of these subjects may have occult coronary artery disease, but this disadvantage was accepted as coronary artery disease in symptom free patients, taking no treatment, has been shown not to influence left ventricular filling (Stewart et al 1992).

No significant abnormalities in plasma fibrinogen, fibrin D-dimer fragment and von Willebrand factor are found in patients with diastolic dysfunction when compared to those without diastolic dysfunction. This finding suggests that the increased fibrinogen, intravascular fibrin turnover and endothelial dysfunction in patients with left ventricular aneurysms are likely to be due to abnormalities of flow in systolic dysfunction, with no significant effect from diastolic dysfunction. This is in keeping with my findings in chapter 8.

Nevertheless, some relationships are seen between these prothrombotic factors and certain Doppler indices of flow. For example, in patients with ischaemic heart disease but normal systolic and diastolic function (Group 1b), there is a significant negative correlation between plasma D-dimer levels, atrial filling rate and atrial ejection force (which is an index of atrial systolic function). This suggests that with higher atrial filling rates and greater atrial systolic function (and thus, less intra-atrial stasis), plasma D-dimer levels would be lower, possibly reflecting less intravascular fibrin turnover and thrombogenesis. Conversely, 
impaired atrial systolic function (that is, lower atrial ejection force) may result in greater intraatrial stasis and thus, greater thrombogenesis (as reflected by higher plasma D-dimer levels). This relationship is however altered by the presence of a left ventricular aneurysm with associated diastolic dysfunction (Group 3a). In this group, there is a positive correlation between plasma D-dimer and atrial filling rate or atrial ejection force, suggesting that the presence of an aneurysm influences the relationship between haemodynamics and thrombogenesis. An aneurysm is however associated with both diastolic and systolic bulging, which results in severe stasis of blood and a consequent predisposition to thrombus formation. A greater atrial filling rate and atrial ejection force (during ventricular diastole) may therefore contribute to diastolic bulging of the aneurysm, thus increasing stasis (particularly at the dyskinetic segment) and promoting thrombogenesis in such patients.

Endothelial dysfunction may also be related to abnormalities of diastolic function seen in ischaemic heart disease. For example, in patients with diastolic dysfunction but normal systolic function (Group 1a), an inverse relationship between vWF levels and passive ventricular filling was noted (as reflected by the negative correlation between vWF and $E_{V \max }$ and $E_{i n t}$ in this group: $r=-0.42, p=0.06$ ). Although this relationship was not demonstrable in Group 2 (systolic dysfunction but no aneurysm), a negative correlation between vWF and peak and atrial filling rates is found. The findings in Group 1a suggest that endothelial (and perhaps endocardial) dysfunction (as reflected by higher vWF levels) could be associated with impaired early ventricular filling during diastole, in the presence of the poor left ventricular compliance associated with diastolic dysfunction (but normal systolic function). In contrast, Group 3a (aneurysms with diastolic dysfunction) patients demonstrate a positive correlation between vWF and $E_{V \max }(r=0.58, p=0.012)$. The presence of an aneurysm may therefore have complex influences on the relationship between endothelial dysfunction and the haemodynamics of left ventricular filling.

In conclusion, this study provides further evidence for the high prevalence of diastolic dysfunction in association with coronary artery disease, occurring in over $60 \%$ irrespective of left ventricular systolic impaiment. Proper assessment of diastolic function is important particularly for patients with cardiac failure. There is, however, no evidence of a 
significant additional contribution to thrombogenesis (as assessed by plasma fibrinogen, von Willebrand factor and fibrin D-dimer) for patients with diastolic dysfunction. Endothelial dysfunction (as assessed by WWF levels) is also similar in patients with and without diastolic dysfunction. A relationship is noted between some prothrombotic factors and Doppler indices of flow which suggests that a relationship exists between cardiac haemodynamics and thrombogenesis. 
TABLE 9.1

LEFT VENTRICULAR DIASTOLIC DYSFUNCTION: PATIENT CHARACTERISTICS AND DOPPLER PARAMETERS (WHOLE GROUP)

\begin{tabular}{|c|c|c|c|}
\hline GROUP 1 & GROUP 2 & GROUP 3 & HEALTHY \\
\hline normal LI & $\begin{array}{l}\text { LV systolic } \\
\text { dysfunction }\end{array}$ & LV an & \\
\hline
\end{tabular}

\begin{tabular}{|c|c|c|c|c|}
\hline total studied & 42 & 34 & 30 & 14 \\
\hline $\begin{array}{c}\text { AGE } \\
\text { mean (s.e.m.) }\end{array}$ & $\begin{array}{l}57.2 \\
(1.4)\end{array}$ & $\begin{array}{l}58.4 \\
(1.4)\end{array}$ & $\begin{array}{l}57.0 \\
(1.9)\end{array}$ & $\begin{array}{l}53.2 \\
(2.2)\end{array}$ \\
\hline$M: F$ & $33: 9$ & 29:5 & $19: 11$ & $8: 6$ \\
\hline $\begin{array}{l}\text { body mass } \\
\text { index } \\
\text { median (IQR) }\end{array}$ & $\begin{array}{c}39.5 \\
(37.3-43.1)\end{array}$ & $\begin{array}{c}40 \\
(38.0-42.3)\end{array}$ & $\begin{array}{c}38.4 \\
(36.9-40.7)\end{array}$ & $\cdots$ \\
\hline smokers & 8 & 10 & 5 & $\ldots$ \\
\hline $\begin{array}{l}\text { Dopplers } \\
\text { studied } \\
\text { (n) }\end{array}$ & 40 & 31 & 30 & 14 \\
\hline $\begin{array}{l}E_{V \max } \\
\mathrm{cm} / \mathrm{s} \\
(\text { IQR) }\end{array}$ & $\begin{array}{c}49.3 \\
(43.8-61.1)\end{array}$ & $\begin{array}{c}51.8 \\
(38.2-62.8)\end{array}$ & $\begin{array}{c}52.1 \\
(40.1-68.2)\end{array}$ & 52.1 \\
\hline $\begin{array}{l}A V \max \\
\mathrm{cm} / \mathrm{s} \\
(\mathrm{IQR})\end{array}$ & $\begin{array}{c}55.3^{\star} \\
(44.9-65.8)\end{array}$ & $\begin{array}{c}51.8 \\
(44.2-70.8)\end{array}$ & $\begin{array}{c}62.1 \\
(48.4-70.3)\end{array}$ & 47.6 \\
\hline $\begin{array}{c}\text { EVmax }_{\text {max }} / \\
\text { Avmax }_{\text {ratio }} \\
\text { (IQR) }\end{array}$ & $\begin{array}{c}0.905^{\star} \\
(0.79-1.10)\end{array}$ & $\begin{array}{c}0.87 \\
(0.73-1.02)\end{array}$ & $\begin{array}{c}0.83 \\
(0.64-1.18)\end{array}$ & 1.055 \\
\hline $\begin{array}{c}\text { E integral } \\
\text { cm } \\
\text { (IQR) }\end{array}$ & $\begin{array}{c}7.42 \\
(6.03-8.74)\end{array}$ & $\begin{array}{c}7.35 \\
(5.92-9.26)\end{array}$ & $\begin{array}{c}6.78 \\
(5.28-8.89)\end{array}$ & 7.12 \\
\hline $\begin{array}{c}\text { A integral } \\
\mathrm{cm} \\
\text { (IQR) }\end{array}$ & $\begin{array}{l}5.4^{*} \\
(3.99-6.32)\end{array}$ & $\begin{array}{c}4.9 \\
(4.03-6.90)\end{array}$ & $\begin{array}{c}5.75 \\
(4.27-6.96)\end{array}$ & 3.97 \\
\hline $\begin{array}{c}\text { E/A integral } \\
\text { ratio } \\
\text { (IQR) }\end{array}$ & $\begin{array}{c}1.38^{\star} \\
(1.15-1.79)\end{array}$ & $\begin{array}{c}1.28 \\
(1.13-6.64)\end{array}$ & $\begin{array}{c}1.31 \\
(0.8-2.0)\end{array}$ & 1.72 \\
\hline
\end{tabular}




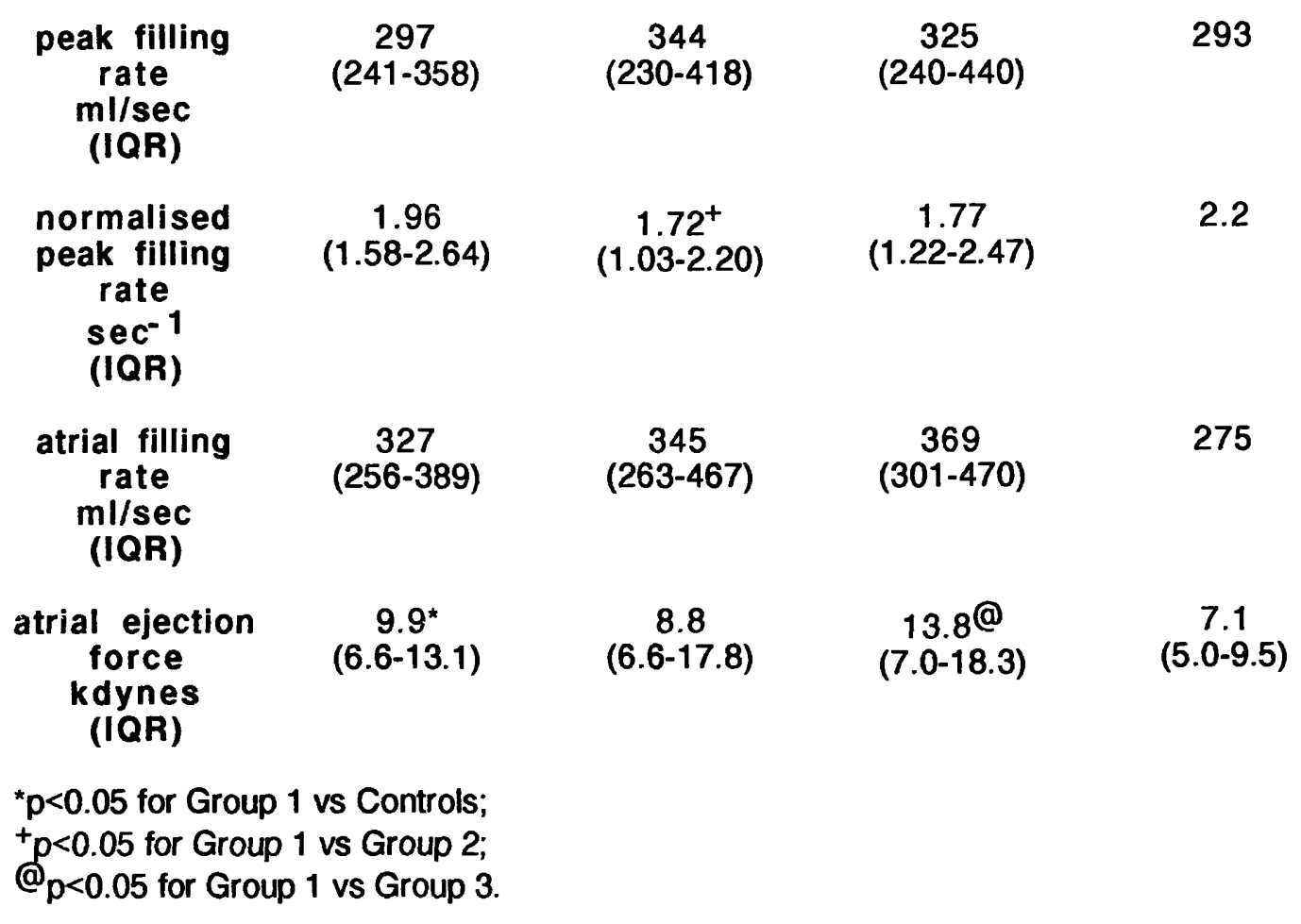




\section{TABLE 9.2}

\section{DIASTOLIC DYSFUNCTION}

\section{\& THROMBOGENIC FACTORS}

\begin{tabular}{|c|c|c|c|c|c|c|}
\hline & $\begin{array}{c}\text { GROUP } \\
\text { NORMAL } \\
1 \text { a } \\
\text { diastolic } \\
\text { dysfunction }\end{array}$ & 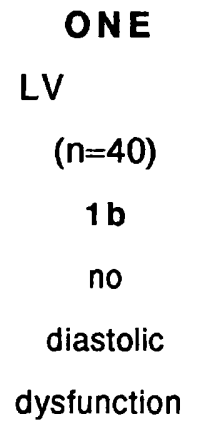 & $\begin{array}{l}\text { GROUP } \\
\text { LV DYS } \\
\text { 2a } \\
\text { diastolic } \\
\text { dysfunction }\end{array}$ & 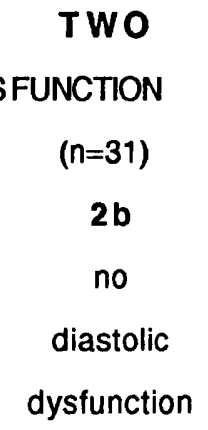 & $\begin{array}{l}\text { GROUP } \\
\text { LVAN } \\
\text { 3a } \\
\text { diastolic } \\
\text { dysfunction }\end{array}$ & $\begin{array}{c}\text { THREE } \\
\text { JEURYSM } \\
\begin{array}{c}(n=30) \\
3 b \\
\text { no }\end{array} \\
\text { diastolic } \\
\text { dysfunction }\end{array}$ \\
\hline $\begin{array}{c}\text { number } \\
(\%)\end{array}$ & $\begin{array}{c}24 \\
(60 \%)\end{array}$ & $\begin{array}{c}16 \\
(40 \%)\end{array}$ & $\begin{array}{c}19 \\
(59.4 \%)\end{array}$ & $\begin{array}{c}13 \\
(40.6 \%)\end{array}$ & $\begin{array}{c}18 \\
(60 \%)\end{array}$ & $\begin{array}{c}12 \\
(40 \%)\end{array}$ \\
\hline $\begin{array}{c}\text { AGE } \\
\text { mean } \\
\text { (s.e.m.) }\end{array}$ & $\begin{array}{l}59.5 \\
(1.6)\end{array}$ & $\begin{array}{l}54.0 \\
(2.53)\end{array}$ & $\begin{array}{c}58 \\
(1.7)\end{array}$ & $\begin{array}{l}58.7 \\
(2.7)\end{array}$ & $\begin{array}{l}59.5 \\
(1.7)\end{array}$ & $\begin{array}{l}57.0 \\
(3.8)\end{array}$ \\
\hline Evmax & $\begin{array}{c}47.6 \\
(40.7-54.6)\end{array}$ & $\begin{array}{c}61.8^{*} \\
(50.0-66.8)\end{array}$ & $\begin{array}{c}45.3 \\
(35.4-59.5)\end{array}$ & $\begin{array}{c}57.7^{+} \\
(51.3-68.5)\end{array}$ & $\begin{array}{c}42.9 \\
(38.4-52.0)\end{array}$ & $\begin{array}{c}70.1 @ \\
(55.0-92.0)\end{array}$ \\
\hline A vmax & $\begin{array}{c}60.5 \\
(53.0-68.7)\end{array}$ & $\begin{array}{c}48.95^{\star} \\
(40.7-56.2)\end{array}$ & $\begin{array}{c}59.9 \\
(49.6-71.2)\end{array}$ & $\begin{array}{c}47.6^{+} \\
(36.5-53.2)\end{array}$ & $\begin{array}{c}68.9 \\
(60.2-73.9)\end{array}$ & $\begin{array}{c}46.4 @ \\
(42.1-62.3)\end{array}$ \\
\hline $\begin{array}{l}E_{v \max } I \\
A_{V \max }\end{array}$ & $\begin{array}{c}0.80 \\
(0.69-0.87)\end{array}$ & $\begin{array}{c}1.14^{*} \\
(1.07-1.32)\end{array}$ & $\begin{array}{c}0.76 \\
(0.7-0.86)\end{array}$ & $\begin{array}{c}1.02^{+} \\
(1.01-1.55)\end{array}$ & $\begin{array}{c}0.67 \\
(0.56-0.79)\end{array}$ & $\begin{array}{c}1.21 @ \\
(1.16-1.60)\end{array}$ \\
\hline E int & $\begin{array}{c}6.82 \\
(5.7-8.31)\end{array}$ & $\begin{array}{c}8.25^{\star} \\
(6.48-9.2)\end{array}$ & $\begin{array}{c}6.4 \\
(4.9-8.4)\end{array}$ & $\begin{array}{c}8.8^{+} \\
(7.4-12.5)\end{array}$ & $\begin{array}{c}5.91 \\
(4.38-7.21)\end{array}$ & $\begin{array}{c}9.06 @ \\
(6.6-10.9)\end{array}$ \\
\hline$A$ int & $\begin{array}{c}6.0 \\
(4.9-6.6)\end{array}$ & $\begin{array}{c}4.0^{\star} \\
(3.75-5.43)\end{array}$ & $\begin{array}{c}5.81 \\
(4.6-6.9)\end{array}$ & $\begin{array}{c}4.2 \\
(3.8-5.8)\end{array}$ & $\begin{array}{c}6.1 \\
(5.6-7.4)\end{array}$ & $\begin{array}{c}4.25 @ \\
(2.9-5.3)\end{array}$ \\
\hline Eint / Aint & $\begin{array}{c}1.2 \\
(1.03-1.37)\end{array}$ & $\begin{array}{c}1.8^{\star} \\
(1.6-1.9)\end{array}$ & $\begin{array}{c}1.16 \\
(0.97-1.28)\end{array}$ & $\begin{array}{c}1.73^{+} \\
(1.35-3.03)\end{array}$ & $\begin{array}{c}0.88 \\
(0.72-1.29)\end{array}$ & $\begin{array}{c}2.13 @ \\
(1.5-2.4)\end{array}$ \\
\hline
\end{tabular}

${ }^{*} p<0.05 ;+p<0.05 ; @ p<0.05$ 


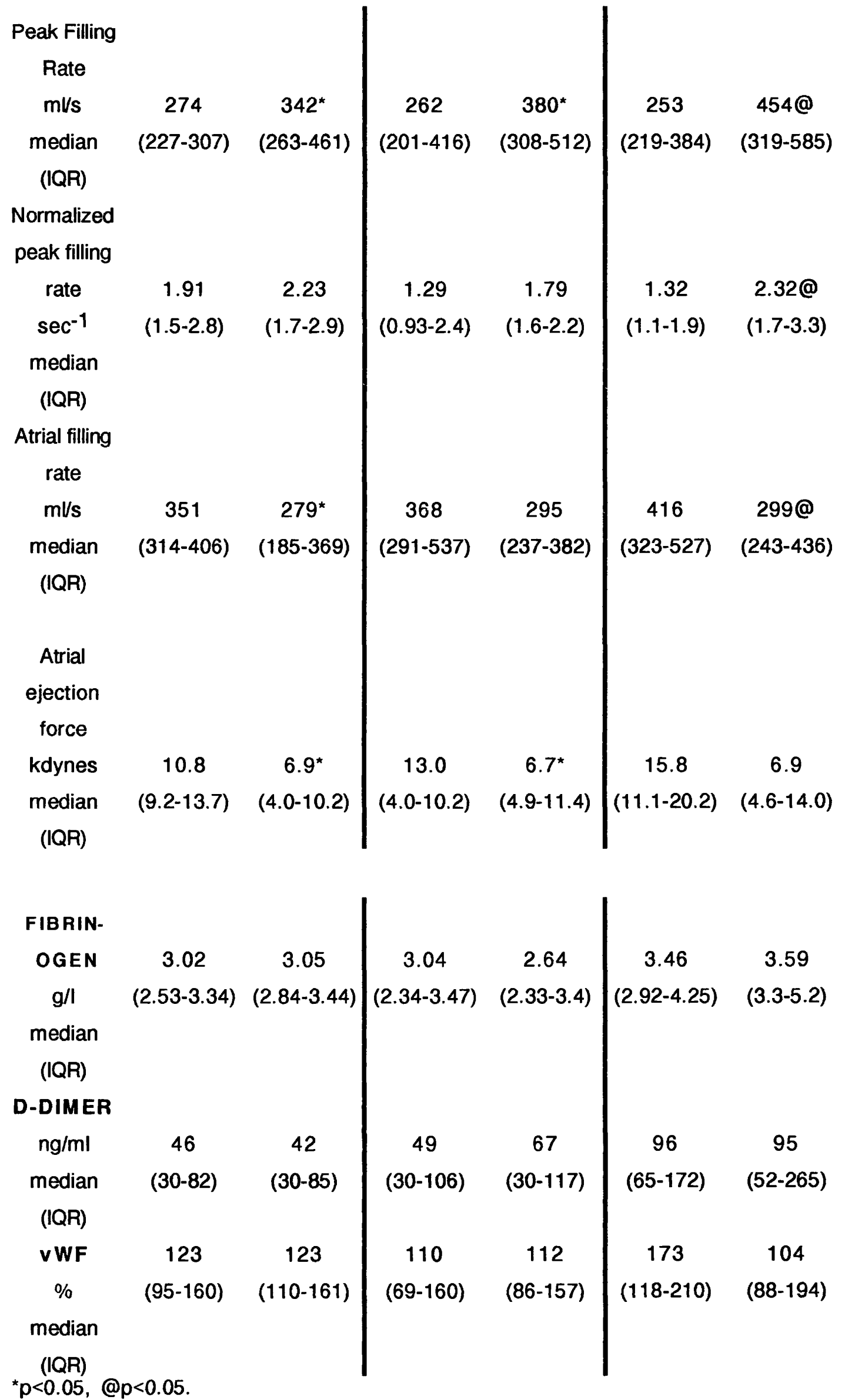


TABLE 9.3

Spearman correlations with Doppler characteristics of diastolic dysfunction

\section{GROUP 1 NORMAL LV SYSTOLIC FUNCTION}

(a) diastolic dysfunction

\begin{tabular}{|c|c|c|c|c|c|c|c|c|}
\hline \multirow[b]{3}{*}{ fibrinogen } & \multicolumn{2}{|c|}{ AGE } & \multicolumn{2}{|c|}{ FIBRINOGEN } & \multicolumn{2}{|c|}{ D- DIMER } & \multicolumn{2}{|l|}{ vWF } \\
\hline & $r$ & $p$ & $r$ & $p$ & $r$ & $p$ & $r$ & p \\
\hline & -0.10 & 0.64 & ... & & $0.43^{*}$ & 0.035 & 0.24 & 0.304 \\
\hline D-dimer & 0.185 & 0.609 & $0.43^{*}$ & 0.035 & $\ldots$ & & 0.012 & 0.957 \\
\hline vWF & -0.35 & 0.122 & 0.24 & 0.304 & 0.012 & 0.957 & $\cdots$ & \\
\hline pfr & 0.27 & 0.19 & 0.147 & 0.5 & -0.25 & 0.239 & -0.09 & 0.706 \\
\hline npfr & 0.34 & 0.126 & 0.193 & 0.59 & -0.03 & 0.877 & -0.41 & 0.098 \\
\hline afr & 0.279 & 0.184 & 0.127 & 0.56 & -0.19 & 0.61 & 0.158 & 0.512 \\
\hline aef & 0.19 & 0.63 & .08 & 0.70 & -0.27 & 0.20 & -0.12 & 0.63 \\
\hline$E_{\text {max }}$ & 0.079 & 0.713 & 0.205 & 0.66 & -0.15 & 0.51 & -0.42 & 0.06 \\
\hline Avmax & 0.047 & 0.822 & 0.104 & 0.63 & -0.18 & 0.599 & -0.26 & 0.277 \\
\hline$E$ int & 0.02 & 0.898 & 0.182 & 0.603 & 0.001 & 0.99 & -0.42 & 0.061 \\
\hline$A$ int & 0.22 & 0.3 & 0.28 & 0.178 & 0.234 & 0.27 & -0.32 & 0.165 \\
\hline$E / A$ & -0.16 & 0.542 & -0.03 & 0.877 & -0.26 & 0.22 & -0.03 & 0.91 \\
\hline E/A int & -0.07 & 0.736 & 0.248 & 0.241 & -0.09 & 0.68 & -0.17 & 0.51 \\
\hline
\end{tabular}

${ }^{*} p<0.05$

[pfr=peak filling rate; $\mathrm{npfr}=$ normalised peak filling rate; afr=atrial filling rate;

aef=atrial ejection force;

$E v_{\max }=$ maximum $E$ wave velocity; $A v_{\max }=$ maximum $A$ wave velocity;

Eint = velocity-time integral of $E$ wave; $A$ int $=$ velocity-time integral of $A$ wave;

$E / A$ vel = ratio of maximum $E$ wave velocity to $A$ wave velocity;

$E / A$ int $=$ ratio of velocity-time integral of $E$ wave to velocity-time integral of $A$ wave] 


\section{GROUP 1}

(b) no diastolic dysfunction

(normal systolic and diastolic function)

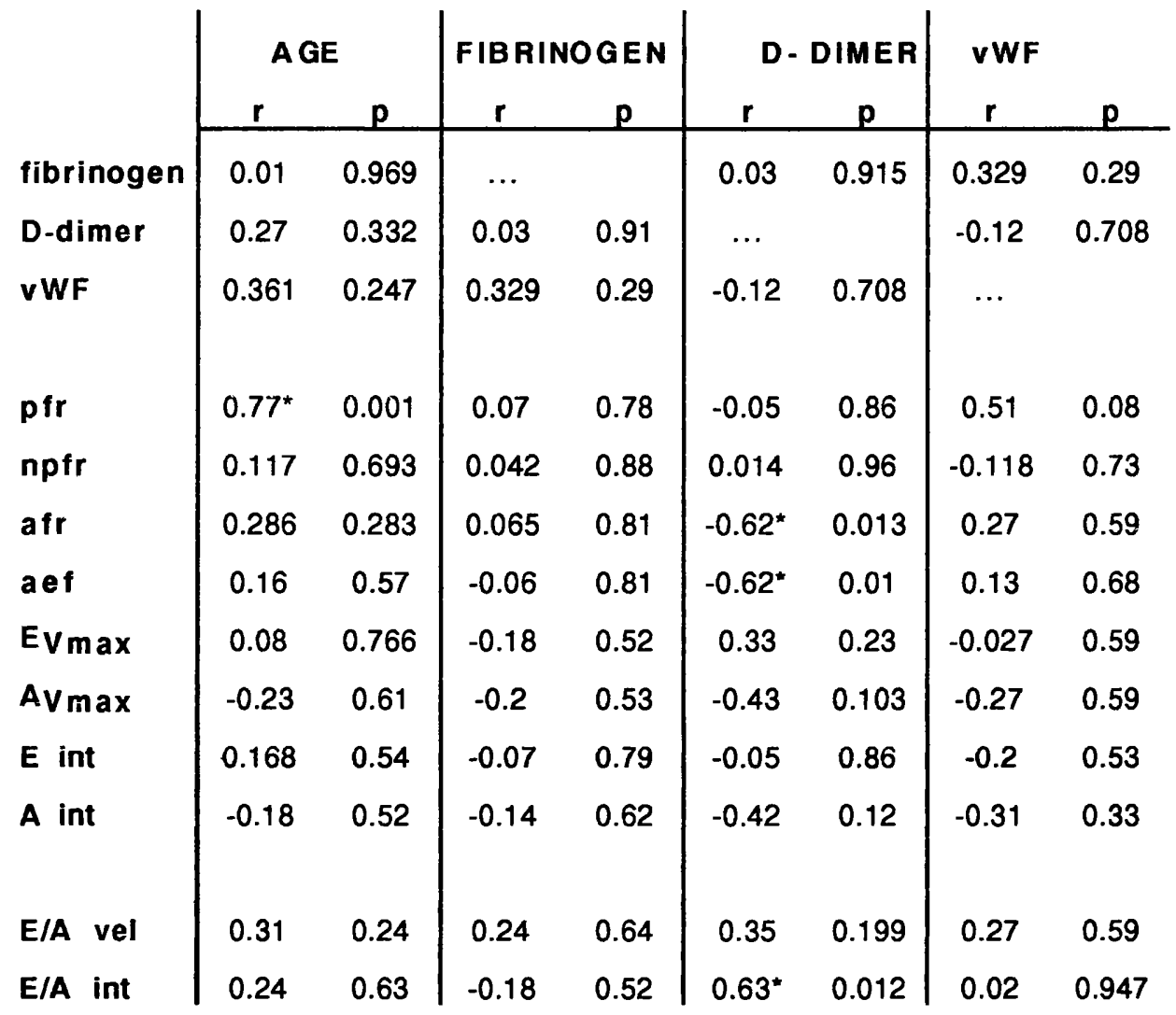

${ }^{*} p<0.05$

[pfr=peak filling rate; npfr=normalised peak filling rate; afr=atrial filling rate;

aef=atrial ejection force;

$E v_{\max }=$ maximum $E$ wave velocity; $A v_{\max }=$ maximum $A$ wave velocity;

Eint = velocity-time integral of $E$ wave; $A$ int = velocity-time integral of $A$ wave;

$E / A$ vel = ratio of maximum $E$ wave velocity to $A$ wave velocity;

$E / A$ int $=$ ratio of velocity-time integral of $E$ wave to velocity-time integral of $A$ wave] 


\section{GROUP 2 LV SYSTOLIC DYSFUNCTION}

(a) diastolic dysfunction

\begin{tabular}{l|cc|cc|cc|cc} 
& \multicolumn{2}{|c|}{ AGE } & & \multicolumn{2}{|c|}{ FIBRINOGEN } & \multicolumn{2}{|c|}{ D- DIMER } & vWF \\
\cline { 2 - 8 } fibrinogen & $\mathbf{r}$ & $\mathbf{p}$ & $\mathbf{r}$ & $\mathbf{p}$ & $\mathbf{r}$ & $\mathbf{p}$ & $\mathbf{r}$ & $\mathbf{p}$ \\
\cline { 2 - 8 } D-dimer & -0.05 & 0.85 & $\ldots$ & & 0.16 & 0.53 & $-0.50^{*}$ & 0.034 \\
VWF & $0.56^{*}$ & 0.01 & 0.16 & 0.53 & $\ldots$ & & -0.22 & 0.61 \\
& -0.03 & 0.898 & $-0.50^{*}$ & 0.034 & -0.22 & 0.61 & $\ldots$ & \\
Pfr & & & & & & & & \\
npfr & 0.32 & 0.18 & 0.22 & 0.63 & 0.25 & 0.31 & $-0.57^{*}$ & 0.014 \\
afr & 0.07 & 0.8 & 0.50 & 0.057 & 0.19 & 0.51 & -0.30 & 0.30 \\
aef & 0.44 & 0.06 & 0.1 & 0.68 & 0.33 & 0.16 & $-0.47^{*}$ & 0.046 \\
EVmax & 0.32 & 0.18 & 0.22 & 0.62 & 0.18 & 0.54 & -0.46 & 0.054 \\
AVmax & 0.06 & 0.79 & 0.37 & 0.12 & 0.01 & 0.96 & -0.41 & 0.09 \\
E int & 0.18 & 0.53 & 0.36 & 0.13 & 0.07 & 0.77 & -0.41 & 0.09 \\
A int & -0.14 & 0.59 & 0.20 & 0.59 & -0.16 & 0.52 & -0.15 & 0.57 \\
& 0.06 & 0.81 & 0.03 & 0.89 & -0.20 & 0.59 & -0.10 & 0.69 \\
E/A vel & & & & & & & & \\
E/A int & -0.26 & 0.29 & 0.37 & 0.12 & -0.01 & 0.97 & -0.16 & 0.54 \\
& 0.04 & 0.85 & 0.24 & 0.33 & 0.09 & 0.72 & -0.27 & 0.27
\end{tabular}

${ }^{*} p<0.05$

[pfr=peak filling rate; $\mathrm{npfr}=$ normalised peak filling rate; afr=atrial filling rate; aef=atrial ejection force;

$E v_{\max }=$ maximum $E$ wave velocity; $A v_{\max }=\operatorname{maximum} A$ wave velocity;

Eint $=$ velocity-time integral of $E$ wave; $A$ int $=$ velocity-time integral of $A$ wave;

$E / A$ vel = ratio of maximum $E$ wave velocity to $A$ wave velocity;

$E / A$ int $=$ ratio of velocity-time integral of $E$ wave to velocity-time integral of $A$ wave] 
GROUP 2

(b) no diastolic dysfunction

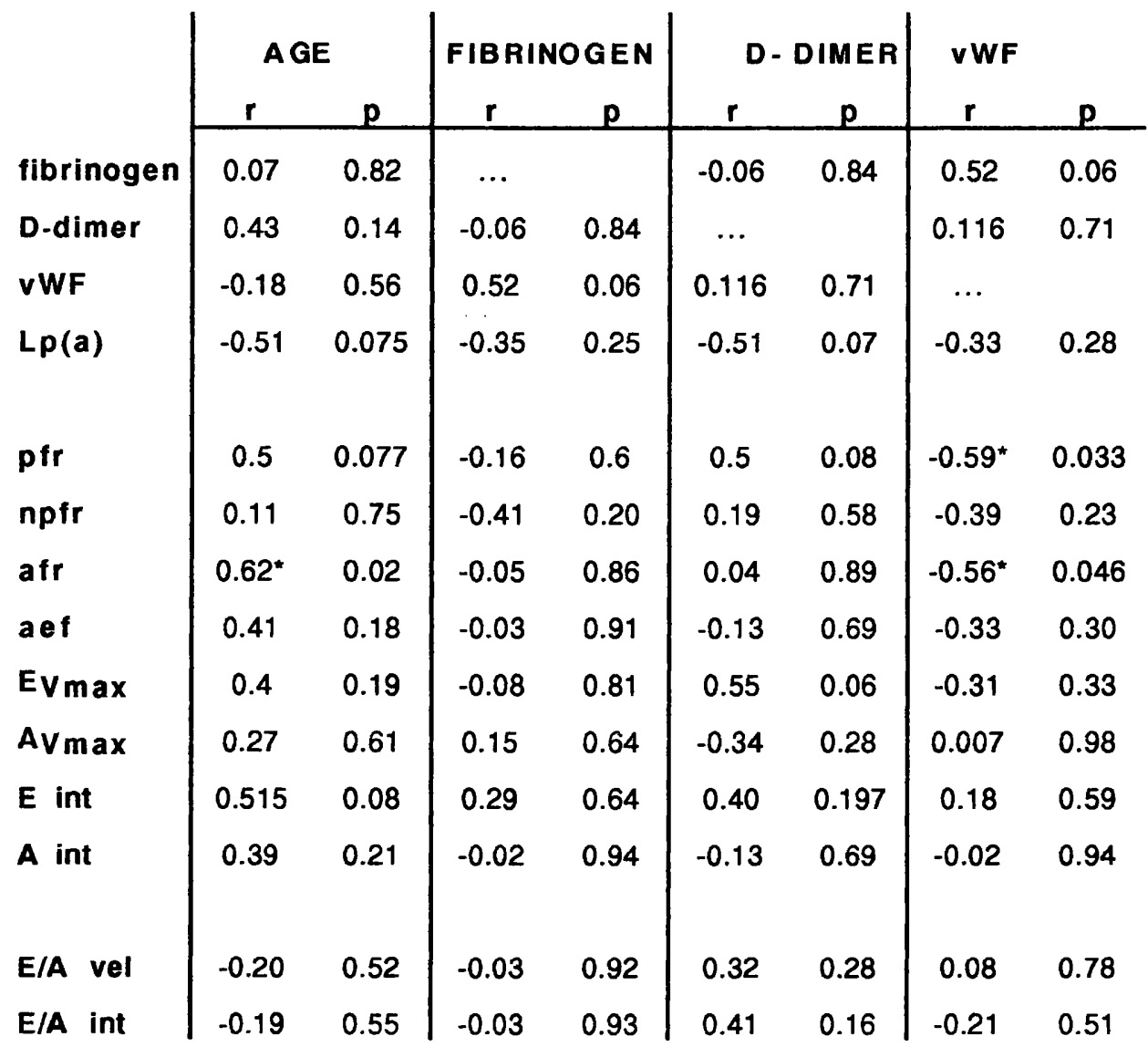

${ }^{*} p<0.05$

[pfr=peak filling rate; npfr=normalised peak filling rate; afr=atrial filling rate; aef=atrial ejection force;

$E v_{\max }=$ maximum $E$ wave velocity; $A v_{\max }=$ maximum $A$ wave velocity;

Eint = velocity-time integral of $E$ wave; $A$ int = velocity-time integral of $A$ wave;

$E / A$ vel = ratio of maximum $E$ wave velocity to $A$ wave velocity;

$E / A$ int = ratio of velocity-time integral of $E$ wave to velocity-time integral of $A$ wave] 


\section{GROUP 3 LV ANEURYSMS}

(a) diastolic dysfunction

\begin{tabular}{l|cc|cc|cc|cc} 
& \multicolumn{2}{|c|}{ AGE } & & \multicolumn{2}{|c|}{$F$ IBRINOGEN } & \multicolumn{2}{|c|}{ D- DIMER } & vWF \\
& $\mathbf{r}$ & $\mathbf{p}$ & $\mathbf{r}$ & $\mathbf{p}$ & $\mathbf{r}$ & $\mathbf{p}$ & $\mathbf{r}$ & $\mathbf{p}$ \\
\cline { 2 - 8 } fibrinogen & 0.066 & .79 & $\ldots$ & & 0.312 & 0.21 & 0.11 & 0.066 \\
D-dimer & 0.226 & 0.63 & 0.312 & 0.21 & $\ldots$ & & 0.35 & 0.149 \\
vWF & 0.03 & 0.90 & 0.44 & 0.066 & 0.35 & 0.149 & $\ldots$ & \\
& & & & & & & & \\
pfr & 0.09 & 0.72 & -0.11 & 0.67 & 0.24 & 0.65 & 0.21 & 0.59 \\
npfr & -0.02 & 0.96 & -0.09 & 0.77 & -0.35 & 0.237 & 0.40 & 0.17 \\
afr & 0.13 & 0.62 & -0.02 & 0.91 & $0.48^{*}$ & 0.042 & 0.13 & 0.62 \\
aef & 0.30 & 0.23 & 0.04 & 0.88 & $0.51^{*}$ & 0.03 & 0.28 & 0.61 \\
EVmax & 0.069 & 0.78 & 0.112 & 0.66 & 0.01 & 0.97 & $0.575^{*}$ & 0.012 \\
AVmax & 0.46 & 0.05 & 0.19 & 0.54 & 0.30 & 0.22 & 0.37 & 0.125 \\
E int & -0.278 & 0.26 & -0.13 & 0.604 & -0.11 & 0.67 & 0.453 & 0.056 \\
A int & $0.604^{*}$ & 0.008 & 0.30 & 0.219 & 0.18 & 0.52 & 0.31 & 0.21 \\
& & & & & & & & \\
E/A vel & $-0.50^{*}$ & 0.035 & -0.31 & 0.21 & -0.34 & 0.17 & 0.16 & 0.53 \\
E/A int & -0.23 & 0.64 & -0.096 & 0.71 & -0.22 & 0.61 & 0.42 & 0.08 \\
*p<0.05 & & & & & & & &
\end{tabular}

[pfr=peak filling rate; $\mathrm{npfr}=$ normalised peak filling rate; afr=atrial filling rate; aef=atrial ejection force;

$E v_{\max }=$ maximum $E$ wave velocity; $A v_{\max }=$ maximum $A$ wave velocity;

Eint = velocity-time integral of $E$ wave; $A$ int = velocity-time integral of $A$ wave;

$E / A$ vel = ratio of maximum $E$ wave velocity to $A$ wave velocity;

$E / A$ int $=$ ratio of velocity-time integral of $E$ wave to velocity-time integral of $A$ wave] 
GROUP 3

(b) no diastolic dysfunction

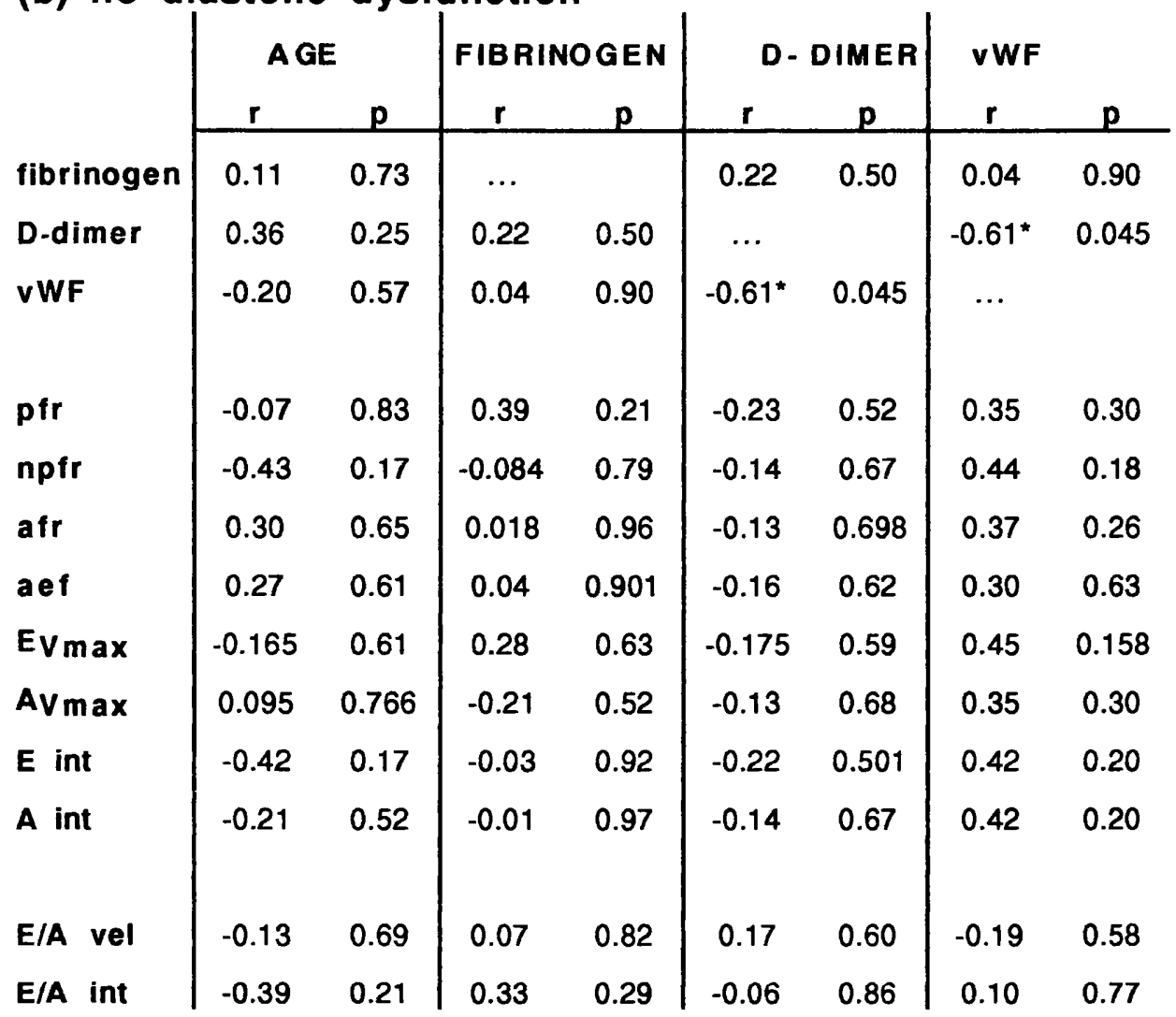

${ }^{\star} p<0.05$

[pfr=peak filling rate; $\mathrm{npfr}=$ normalised peak filling rate; afr=atrial filling rate; aef=atrial ejection force;

$E v_{\max }=$ maximum $E$ wave velocity; $A v_{\max }=$ maximum $A$ wave velocity;

Eint $=$ velocity-time integral of $E$ wave; $A$ int $=$ velocity-time integral of $A$ wave;

$E / A$ vel = ratio of maximum $E$ wave velocity to $A$ wave velocity;

$E / A$ int $=$ ratio of velocity-time integral of $E$ wave to velocity-time integral of $A$ wave] 
TABLE 9.4

EFFECTS OF ANEURYSMS, LV SYSTOLIC DYSFUNCTION AND DIASTOLIC DYSFUNCTION

(AND THEIR INTERACTION) ON PROTHROMBOTIC FACTORS

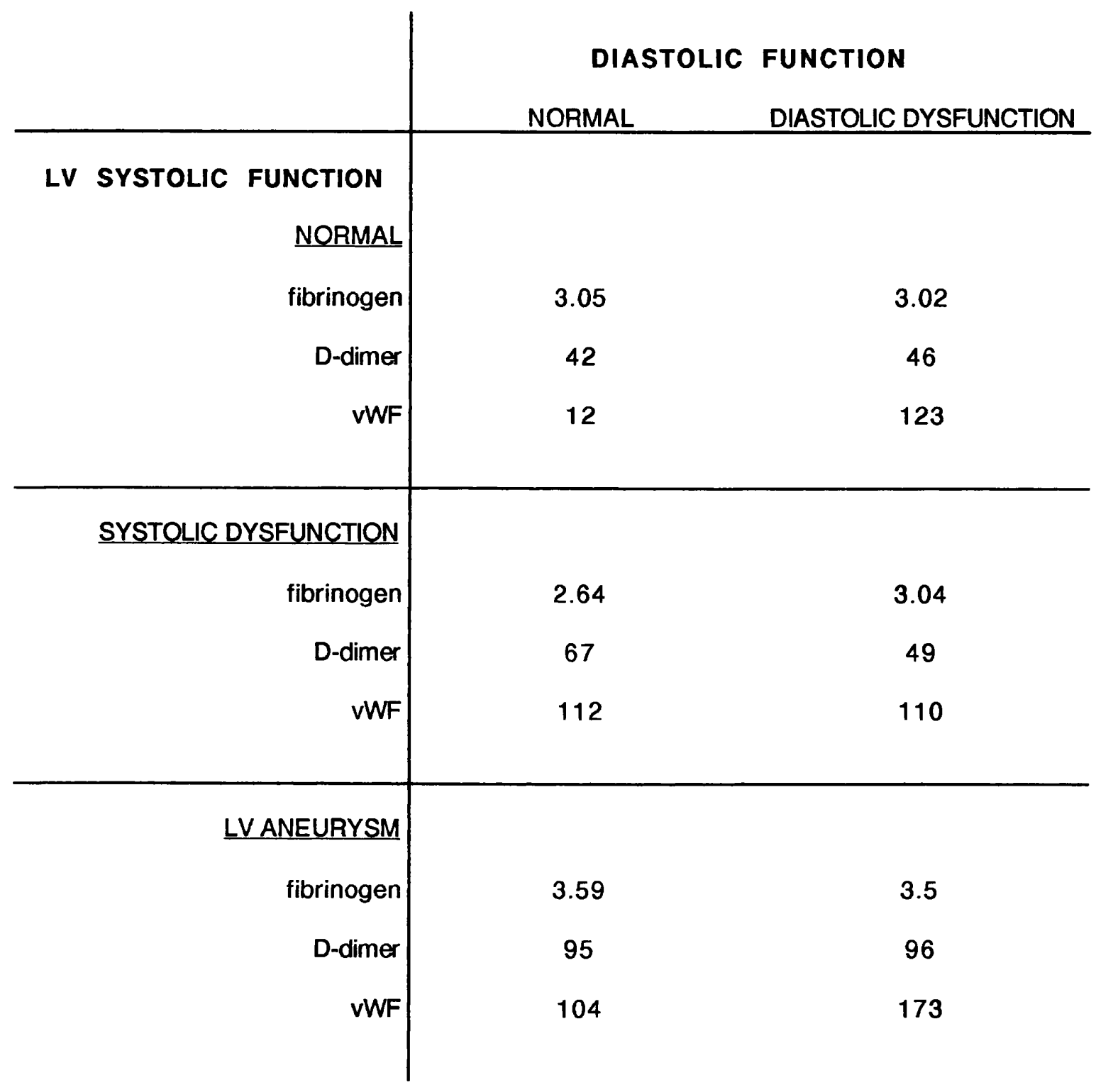

(VWF $=$ von Willebrand factor)

${ }^{*} p<0.05$ for contribution of aneurysms and systolic function for plasma fibrinogen and vWF (GLM-ANOVA) 
TABLE 9.5

INTRA- AND BETWEEN-OBSERVER REPEATABILITY FOR DOPPLER ECHOCARDIOGRAPHY

(a) intra-observer repeatability (IOR)

\begin{tabular}{|c|c|c|c|c|c|}
\hline MEASUREMENT & $\begin{array}{c}\text { Pearson } \\
r\end{array}$ & $\begin{array}{c}\text { mean } \\
\text { difference }\end{array}$ & \pm 2 s.d & $\begin{array}{c}\text { one } \\
\text { sample } \\
\text { t-test T }\end{array}$ & p value \\
\hline$E_{V \max }$ & 0.884 & -0.34 & 3 & -0.90 & 0.38 \\
\hline AVmax & 0.966 & 0.53 & 8 & 0.62 & 0.54 \\
\hline E/A vel ratio & 0.896 & -0.07 & 0.36 & -1.67 & 0.11 \\
\hline E integral & 0.993 & 0.10 & 0.60 & 1.64 & 0.12 \\
\hline A integral & 0.942 & 0.17 & 0.94 & 1.65 & 0.12 \\
\hline E/A int ratio & 0.940 & -0.02 & 0.56 & -0.37 & 0.71 \\
\hline
\end{tabular}

(b) between-observer repeatability (BOR)

\begin{tabular}{c|c|cc|cc} 
MEASUREMENT & $\begin{array}{c}\text { Pearson } \\
\mathbf{r}\end{array}$ & $\begin{array}{c}\text { mean } \\
\text { difference }\end{array}$ & \multicolumn{2}{|c|}{$\begin{array}{c}\text { one } \\
\text { sample } \\
\text { t-test }\end{array}$} & T value \\
\hline & & & & & \\
EVmax & 0.992 & 0.5 & 4 & 1.11 & 0.28 \\
AVmax & 0.990 & 0.98 & 4 & 2.01 & 0.06 \\
E/A vel ratio & 0.978 & -0.01 & 0.14 & -0.90 & 0.38 \\
E integral & 0.996 & 0.07 & 0.4 & 1.59 & 0.13 \\
A integral & 0.984 & 0.10 & 0.52 & 1.76 & 0.09 \\
E/A int ratio & 0.981 & -0.03 & 0.30 & -0.97 & 0.34
\end{tabular}

$p=N S$ 


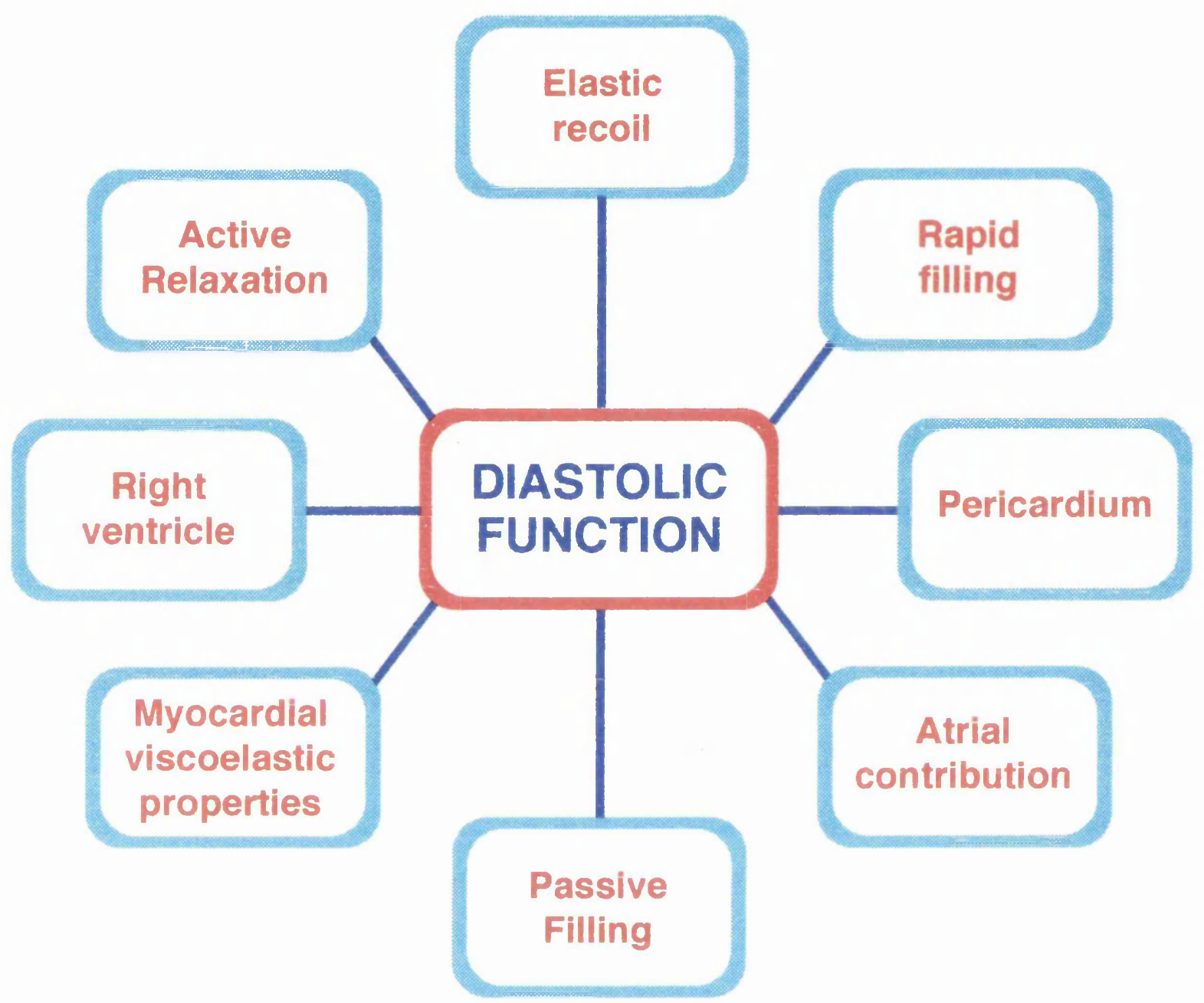

FIGURE 9.1

DETERMINANTS OF LEFT VENTRICULAR DIASTOLIC FUNCTION 
myocardial

infarction (\& scar);

wall motion abnormalities

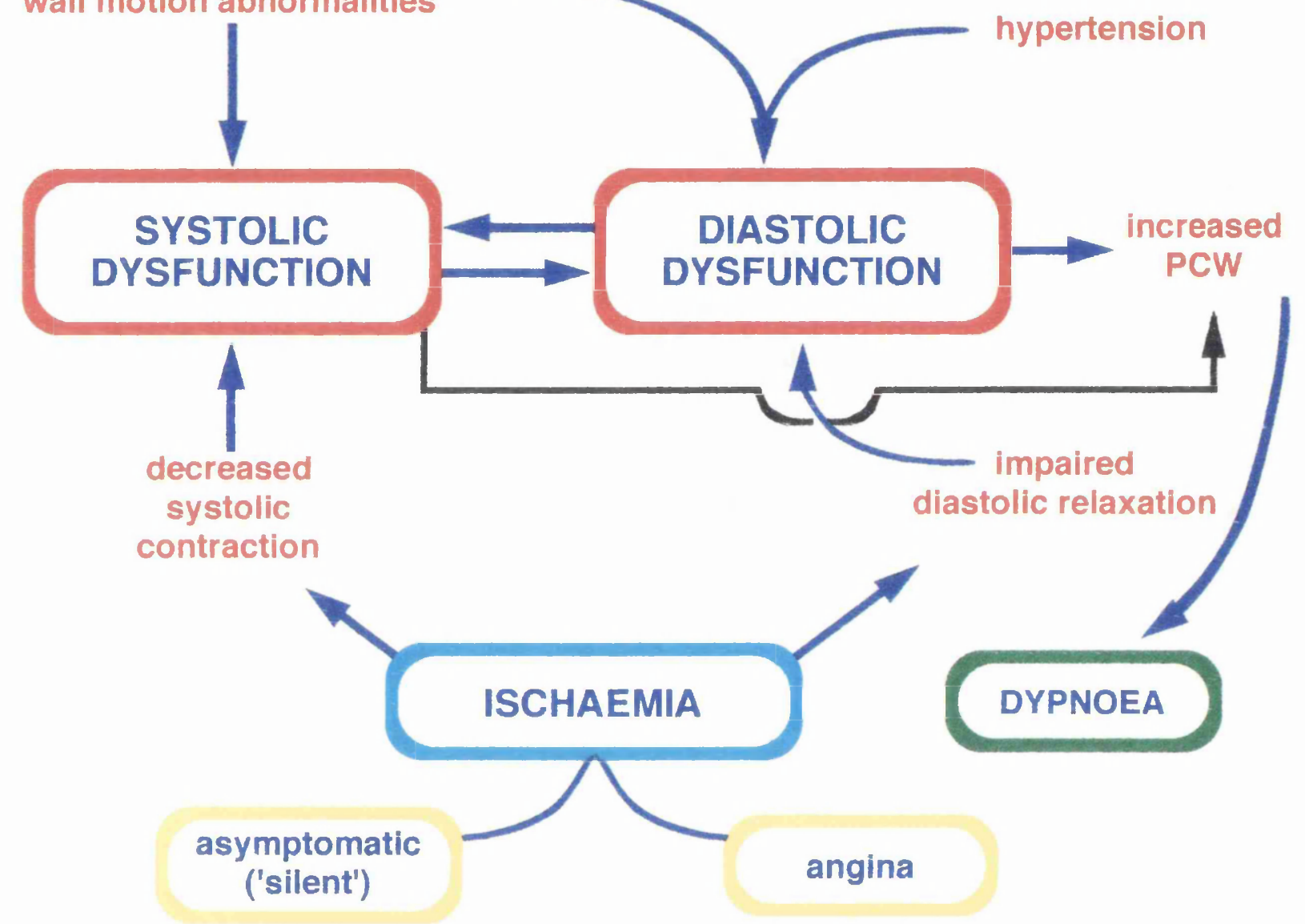

(pcw = pulmonary capillary wedge)

FIGURE 9.2

RELATIONSHIP BETWEEN SYSTOLIC AND DIASTOLIC DYSFUNCTION IN ISCHAEMIC HEART DISEASE 


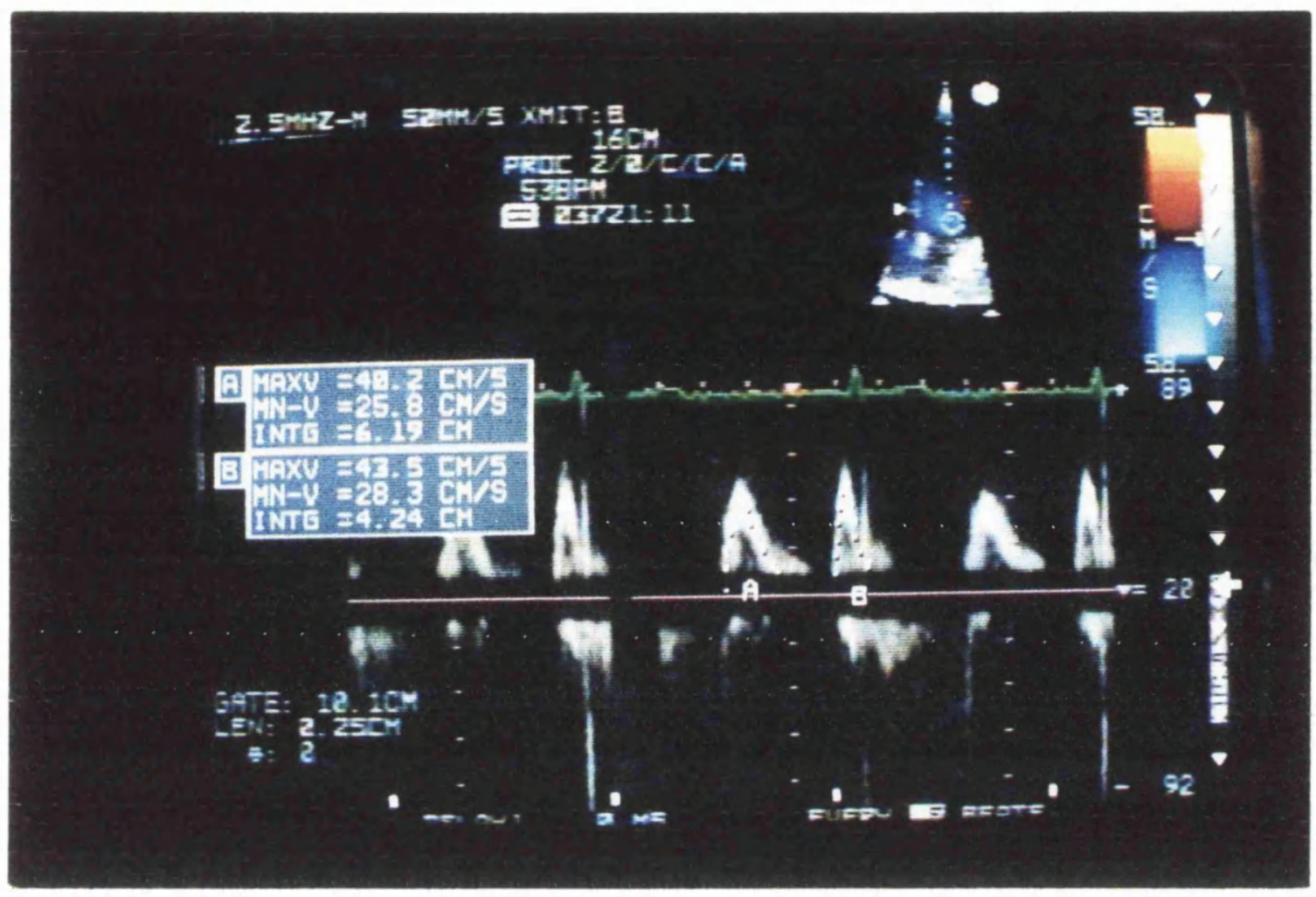

The first peak ( $E$ wave) is due to early passive filling and the second peak (A wave) is due to the atrial contribution to filling.

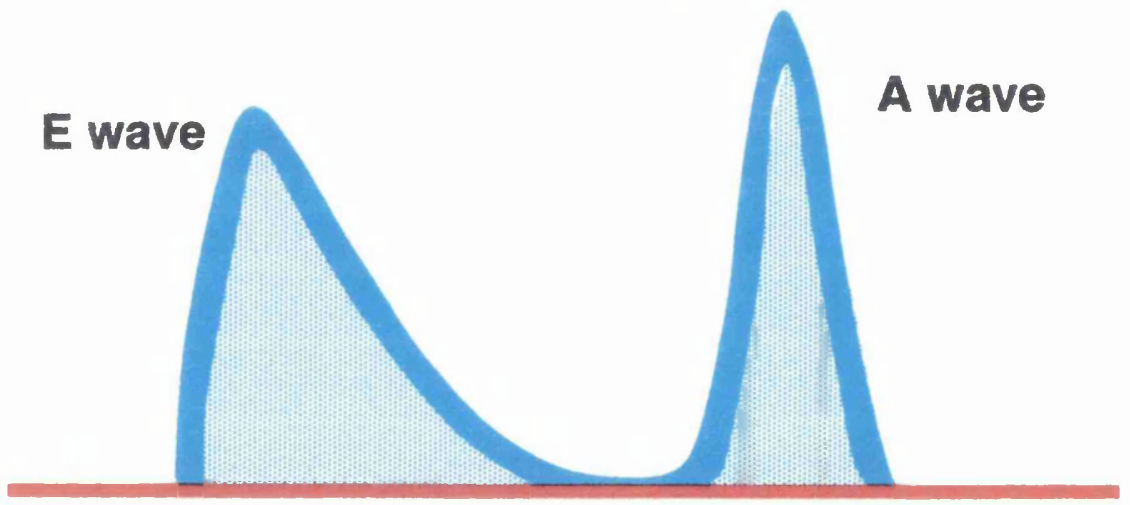

FIGURE 9.3

AN EXAMPLE OF PULSE WAVE DOPPLER ECHOCARDIOGRAPHY

demonstrating mitral inflow velocity pattern

with 'E' or Early (marked 'A' on picture)

and 'A' or Atrial (marked 'B' on picture) waves 
NORMAL

E/A vel

usually

$1.0-2.0$

DIASTOLIC

DYSFUNCTION

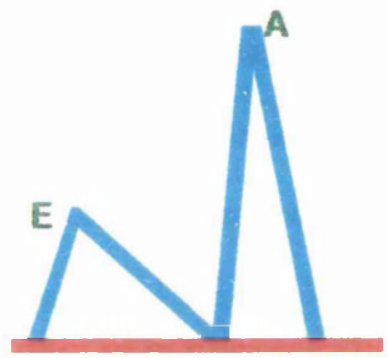

ABNORMAL RELAXATION

E/A vel $<1.0$

- increased IVRT

- increased deceleration time

(IVRT = isovolumetric relaxation time)
DIASTOLIC

DYSFUNCTION

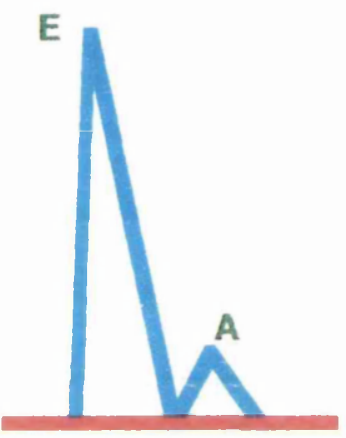

RESTRICTIVE or CONSTRICTIVE

E/A vel >2.0

- decreased IVRT

- decreased deceleration time

FIGURE 9.4

ECHOCARDIOGRAPHIC PATTERNS OF

DIASTOLIC DYSFUNCTION 
CHAPTER 10

\author{
ANTICOAGULATION \\ FOR \\ ATRIAL FIBRILLATION \\ AND \\ LEFT VENTRICULAR \\ DYSFUNCTION
}




\subsection{ANTICOAGULATION FOR CHRONIC ATRIAL FIBRILLATION AND LEFT VENTRICULAR DYSFUNCTION}

\section{ANTICOAGULANT THERAPY IN ATRIAL FIBRILLATION}

Atrial fibrillation has a prevalence of 2 to 5 percent in the general population over the age of 60, with an incidence that increases with age (Chesebro et al 1990). This is pertinent as the presence of this arrhythmia is an important risk for stroke and other thromboembolic events (Lowe 1992, Shimomura et al 1989). For example, non-rheumatic atrial fibrillation has been associated with a fivefold increase in the risk of ischaemic stroke and a five to seven percent yearly risk which also increases with age (Chesebro et al 1990). The thromboembolic risks of atrial fibrillation are illustrated by several large studies. For example, the annual rate of stroke in the Framingham study for patients in atrial fibrillation was approximately $4 \%$ (Wolf et al 1987), a rate which is comparable to two other studies: the Copenhagen AFASAK study and the Stroke Prevention in Atrial Fibrillation Study, where the annual incidence of stroke amongst placebo patients was $4.9 \%$ and $4.9 \%$ respectively (Petersen et al 1989, Stroke Prevention in Atrial Fibrillation Study Group Investigators 1990). In addition, the female sex and other underlying heart disease are contributory factors to the risk of stroke and thromboembolism (Cabin et al 1990, Yamanouchi et al 1989). For example, the embolic risk of atrial fibrillation is 18 times greater if rheumatic heart disease is also present (Cairns and Connolly 1991, Koenig et al 1992). In elderly patients (mean age 79.3), the presence of nonvalvular atrial fibrillation was also an important factor in fatal massive cerebral infarction (Yamanouchi et al 1989).

Although atrial fibrillation remains an established risk factor for thromboembolic events, preventive therapy has, to date, been both empirical and controversial. It is only recently that effective prevention against strokes in atrial fibrillation has been established by prospective clinical studies; this being the use of anticoagulation therapy as prophylaxis against stroke in patients with atrial fibrillation. The purpose of this review is to discuss the evidence for anticoagulation in atrial fibrillation and its place in the management of such patients. 
Contributory factors to stroke in atrial fibrillation

Contributory risk factors for thromboembolic events in atrial fibrillation include the presence of structural heart disease (for example enlarged left atrial size and mitral valve disease), hypertension and poor left ventricular function (Hart 1992). In support of this, multivariate analysis of the largest cohort of placebo-treated patients yielded three independent clinical predictors of an increased risk of stroke: (i) a history of hypertension; (ii) recent (within 3 months) congestive heart failure; and (iii) previous cerebrovascular event (either stroke or transient ischaemic attack) (Stroke Prevention in Atrial Fibrillation Investigators 1992a). Risk stratification on the basis of these three factors allowed the identification of three rates of arterial thromboembolism: $2.5 \%$ (for patients with no risk factors); $7.2 \%$ per year (if one risk factor present); and $17.6 \%$ per year (if 2 or 3 risk factors present) (Stroke Prevention in Atrial Fibrillation Investigators 1992a). Other additive risk factors to the risk of thromboembolism may be the female sex, smoking, heart failure and a history of previous embolic episodes (Hart 1992).

Recent studies have suggested that left atrial enlargement may be consequent upon the duration of atrial fibrillation (Sanfillipo et al 1990, Petersen 1987, SosaSuarez 1989). However, previous evidence has suggested the converse, that left atrial enlargement was the cause rather than a consequence of atrial fibrillation (Takahashi 1982). This is important as the left atrial size has been considered to contribute to an increased risk of atrial thrombi, arterial embolism or stroke in patients with atrial fibrillation. It is also consistent with the observation that the risk of thromboembolism increases with the duration of atrial fibrillation (Cabin et al 1990, Caplan et al 1986, Daniel et al 1988). More specifically, a left atrial size of $\geq 4.0 \mathrm{~cm}$ has been previously identified as the single strongest predictor of increased risk for embolization (Cabin et al 1990). Patients with left atrial enlargement are also associated with the presence of spontaneous echo contrast on transoesophageal echocardiography, suggesting slow or sluggish blood flow within the enlarged atrial cavity (Daniel et al 1988). Spontaneous echo contrast has been associated with the presence of intraatrial thrombi, and indeed, emboli from stasis-related left atrial thrombi are the most likely 
source of thromboembolic events in atrial fibrillation. The rheological mechanism for spontaneous echo contrast is thought to involve the presence of fibrinogen, or its products, and rouleau formation of erythrocytes (Siegel et al 1981).

As mentioned above, the duration and onset of atrial fibrillation may also be of importance. The onset of atrial fibrillation may, for example, be temporally related to the imminence of stroke. In the Framingham study, atrial fibrillation was present at the time of stroke onset in $24 \%$ and about a third of the strokes associated with atrial fibrillation occurred within six months of onset of the arrhythmia (Wolf et al 1983). In addition, stroke recurrence in the first six months following the initial event was more than twice as common in patients with continued atrial fibrillation ( $47 \%$ vs $20 \%$ in non-atrial fibrillation group)(Wolf et al 1983 ).

\section{Clinical studies in anticoagulation for atrial fibrillation}

Until recently, the only evidence supporting the benefit of anticoagulation therapy has been from retrospective studies. One example is the Montreal Heart Institute Study, which found that the incidence of thromboembolic events was eight times more frequent during the period without anticoagulation therapy, whether or not mitral valve disease was present and regardless of whether or not atrial fibrillation was chronic or paroxysmal (Roy et al 1986). In the mid-1980s, five randomised prospective clinical trials were independently initiated to define the value of antithrombotic therapy with warfarin and/or aspirin as prophylaxis in patients with atrial fibrillation. All five studies show consistent results (Table 10.1).

In the Copenhagen Atrial Fibrillation, Aspirin, Anticoagulation Study (AFASAK Study), 1007 patients (540 men, 467 women; median age 74.2 years) with chronic non-rheumatic atrial fibrillation were randomised to receive warfarin, aspirin or placebo (Petersen et al 1989). Warfarin was given openly to 335 patients (median age 72.8 years) and the warfarin dose was adjusted to a prothrombin-time ratio between 1.5 to 1.9 (international normalised ratio (INR) 2.8 to 4.2). Seventy-five milligrams of aspirin once daily was also administered to a further 336 patients (median age 75.1 years). Primary endpoints were thromboembolic events (such as stroke, transient ischaemic attack or systemic embolism), 
and the secondary endpoint was death. In this study, 5 patients on warfarin had thromboembolic complications compared with 20 patients on aspirin and 21 on placebo. There was therefore a significant benefit of warfarin in reducing severe thromboembolic events but only a small (and non-significant) benefit of aspirin (with a risk reduction $60 \%$ and $16 \%$ respectively) (Petersen et al 1989).

The Stroke Prevention in Atrial Fibrillation (SPAF) Study was a multicentre randomised trial in which 1330 patients (34\% of whom had paroxysmal atrial fibrillation) were randomised to two groups (Stroke Prevention in Atrial Fibrillation Study Group Investigators 1990, Stroke Prevention in Atrial Fibrillation Study Group Investigators 1991). Group 1 consisted of 627 patients eligible for anticoagulation, and received a warfarin dose adjusted to aprothrombin-time ratio of 1.3 to 1.8 (equivalent to an international normalised ratio (INR) of 1.7 to 4.6$)$ ( $n=210$ ), aspirin therapy (325mg daily) or placebo. Group 2 consisted of 703 patients not eligible for warfarin, whom were given aspirin $(n=346)$ or placebo in a double-blind study. The end points in this study were ischaemic stroke or systemic embolism. Similar to the AFASAK study, the SPAF study demonstrated a significant reduction of primary events (by $67 \%$ ) by the use of warfarin (warfarin vs placebo $2.3 \%$ vs $7.4 \%$ ). In patients assigned to aspirin there was also a reduction in primary events (by $42 \%$ ) although there was no benefit of aspirin in patients over 75 years of age and severe strokes were not prevented (Stroke Prevention in Atrial Fibrillation Study Group Investigators 1990, Stroke Prevention in Atrial Fibrillation Study Group Investigators 1991). The relative value of warfarin compared to aspirin is unclear and the SPAF II study is continuing to address this critical question, which is further discussed below.

The third major study undertaken addressing this issue was the Boston Area Anticoagulation Trial for Atrial Fibrillation (BAATAF) (Boston Area Anticoagulation Trial for Atrial Fibrillation Investigators 1990). This was an unblinded, randomised controlled trial of 420 patients with nonrheumatic atrial fibrillation: 212 patients were allocated to warfarin and 208 to placebo. The control group in this study could choose to take aspirin at $325 \mathrm{mg}$ daily but there was, however, no significant benefit shown for aspirin (Boston Area Anticoagulation Trial for Atrial Fibrillation Investigators 1990, Singer 1992). There were 2 strokes in the 
warfarin group compared to 13 strokes in control group, a significant risk reduction with warfarin of $86 \%$.

The fourth major clinical study was the Canadian Atrial Fibrillation Anticoagulation (CAFA)Study (Connolly et al 1991). This was a randomised double-blind placebo-controlled trial with objectives similar to the aforementioned studies. However, publication of the AFASAK and SPAF study results, which demonstrated the beneficial effects of anticoagulation resulted in the CAFA study being prematurely terminated before its target recruitment. However in this study of $\mathbf{3 7 8}$ patients (187 warfarin, 191 placebo) there was primary event rate of $3.5 \%$ for the warfarin group compared to $5.2 \%$ for the placebo group, which amounted to a risk reduction of $37 \%$ (Connolly et al 1991). Although this result from the CAFA study was consistent with previously published studies (Petersen 1989, SPAF Investigators 1990, SPAF Investigators 1991, BAATAF Investigators 1990), it did not reach statistical significance ( $95 \%$ confidence interval $-63.5 \%$ to $75.5 \%, p=0.17$ ) (Connolly et al 1991).

The fifth major study of anticoagulation in atrial fibrillation was the Veterans Affairs Stroke Prevention in Nonrheumatic Atrial Fibrillation (SPINAF) study (Ezekowitz et al 1991). This was a randomised, double-blind placebo-controlled study of 571 men with chronic nonrheumatic atrial fibrillation (260 patients on warfarin; 265 placebo). The primary end point was cerebral infarction, whilst secondary end-points were cerebral haemorrhage and death. There were 19 events in the placebo group compared to 4 events in the warfarin group, again a significant risk reduction (of 79\%) (Ezekowitz et al 1991). As in the other studies, there was substantial protection against cerebral infarction in patients with nonrheumatic atrial fibrillation, without an associated excess risk of serious haemorrhage. Additionally, the SPINAF study demonstrated the safety and efficacy of warfarin in patients aged over 70 years, who have the highest prevalence of atrial fibrillation. This study was also fully blinded (whilst its results were consistent with the other, nonblinded studies), which allays concern about the lack of blinding in the other trials. 
Discussion

All five of the atrial fibrillation anticoagulation studies discussed above demonstrate that anticoagulation with warfarin (aiming for a target International Normalised Ratio (INR) of 1.5 to 3.0 , or a target prothrombin-time ratio of 1.2 to 1.5 ) reduces the rate of stroke among patients with atrial fibrillation by two thirds or more. All 5 studies were terminated early by their data-monitoring boards in view of the marked benefit of warfarin therapy in the prevention of thromboembolic events. Remarkably, all five independent contemporaneous trials came to the same consistent conclusion, namely the fact that anticoagulation therapy significantly reduced the risk of stroke in patients with nonrheumatic atrial fibrillation. The rate of bleeding was usually under $1 \%$ per year, despite the study patients being predominantly elderly.

Despite the evidence of the effectiveness of warfarin, the estimate of this preventive efficacy remains imprecise - each trial discussed above had wide confidence intervals. Additionally, further studies are required to define which patients with atrial fibrillation are at the highest risk of stroke and identify which patients do not require prophylactic therapy. Oral anticoagulation therapy does appear to be a relatively safe therapeutic stratagem (although the potential of haemorrhage still exists) and should therefore be standard therapy for all patients with atrial fibrillation, if no contraindications to anticoagulation exist. However, prophylaxis with warfarin has two main problems: inconvenience and safety (Lowe 1992). In the BAATAF study, no significant differences between warfarin-treated and controls were found for quality of life as demonstrated by measures of functional status, well-being, and health perceptions; unless complicated by a bleeding episode (Lancaster et al 1991). Patients on warfarin need, however, regular education and the effect of warfarin must be regularly monitored (and the dose adjusted). Surprisingly, the quality of life assessment of patients on the BAATAF study demonstrated that $75.6 \%$ did not mind regular blood tests (Lancaster et al 1991). The cost effectiveness and safety aspects have also to be considered; in particular, the risk of bleeding. Patients on warfarin who sustain a bleeding complication demonstrate a significant decrease in health perceptions (Lancaster et al 1991). 
Primary prophylaxis with anticoagulant therapy in atrial fibrillation is cost effective if the rate of intracranial bleeding during such therapy is only $0.3 \%$, as in the major published trials (Gustafsson et al 1992). However if this rate increases to $2 \%$ per year, warfarin therapy becomes no longer cost effective (Gustafsson et al 1992). This "cost" has to be balanced against the reduction of the annual risk of stroke from 5\% to $1.8 \%$ (Lowe 1992).

One concern with these five trials is the highly selected nature of the patient population. For example, in the SPAF and SPINAF studies, $93 \%$ of those screened in each study were excluded from entry (Stroke Prevention in Atrial Fibrillation Study Group Investigators 1991, Ezekowitz et al 1991). Similarly, in the Canadian study, only $13 \%$ of those screened were eligible, but of these only $54 \%$ gave consent and were randomised (Connolly et al 1991). Patients' refusal and physicians' refusal to enrol patients in these studies were the most important reasons for exclusion. The patient withdrawal rate was also high, ranging from $19 \%$ to $38 \%$ in four of the trials (Connolly et al 1991 , Ezekowitz et al 1991 , Petersen et al 1989, Stroke Prevention in Atrial Fibrillation Study Group Investigators 1991). There is concern therefore whether or not the results of these studies reflect 'clinical reality' and can be directly translated into clinical practice.

Evaluation of other treatments which are more convenient or less risky is also required. For example, aspirin would be preferable to warfarin if it were equally effective, if only for the ease of administration. The results however remain inconsistent. For example, the AFASAK study demonstrated no benefit from aspirin at $75 \mathrm{mg}$ daily, but this was in an older population (Petersen et al 1989). The Stroke Prevention in Atrial Fibrillation study reported that aspirin at $325 \mathrm{mg}$ daily had some beneficial effect, but not in patients over the age of 75 years, nor was it effective in preventing severe strokes (Stroke Prevention in Atrial Fibrillation Investigators 1990, Stroke Prevention in Atrial Fibrillation Investigators 1991). The BAATAF study also indicated no significant effect of aspirin at $325 \mathrm{mg}$ daily (Boston Area Anticoagulation Trial for Atrial Fibrillation Investigators 1990). The benefit of aspirin when compared to warfarin therapy has therefore been addressed in the SPAF II Study. Preliminary results of 1100 patients randomised to warfarin or aspirin therapy have been presented at the 42nd Annual Scientific Session of the American College of Cardiology (JL Halpern, Anaheim, 
16 March 1993). This study demonstrated that a risk stratification of patients with chronic nonrheumatic atrial fibrillation was possible and that aspirin therapy does appear suitable for certain groups of patients. For example, those patients with chronic atrial fibrillation who appear at highest risk are those who are female, aged over 75 , systolic blood pressure $>160$ $\mathrm{mm} \mathrm{Hg}$ and with echocardiographic evidence of left ventricular impairment. In these patients, aspirin alone is inadequate as prophylaxis (with a relative risk between $2.0-2.8$ for each of the risk factors mentioned) and warfarin is required (JL Halpern, Presentation at the 42nd Annual Scientific Session of the American College of Cardiology, Anaheim, 16 March 1993). However, patients who are males, aged less than 75 years, with systolic blood pressure $<160$ $\mathrm{mm} \mathrm{Hg}$ and no left ventricular impairment are are low risk for thromboembolism, and aspirin $(325 \mathrm{mg})$ therapy alone appears sufficient as prophylaxis. Patients who are aged $<65$ with no other risk factors have a very low thromboembolic risk, and antithrombotic therapy may not be required. Aspirin may be useful in combination with warfarin therapy in the high risk patient group described above and their additive effects, if any, are being studied in the SPAF III Study.

In conclusion, patients with chronic atrial fibrillation appear to benefit from oral anticoagulation as a prophylaxis against thromboembolic events. When carefully managed, warfarin therapy is well tolerated. Aspirin therapy may also have a role for certain patients at lower risk. Anticoagulant therapy is, however, not without risk and the need for a method to identify those individual patients at highest risk is still required. It is possible that the use of certain echocardiographic criteria and suitable markers of thrombogenesis may help distinguish these patients.

\section{ANTICOAGULANT THERAPY IN LEFT VENTRICULAR DYSFUNCTION} Introduction

As left ventricular mural thrombus is often associated with disease involving the endocardium and/or myocardium of the left ventricle, the precise prevalence of left ventricular thrombus is difficult to ascertain. For example, the presence of thrombus within the left ventricle has been associated with the following diseases and clinical states: acute 
myocardial infarction, left ventricular aneurysm, congestive cardiomyopathy, blunt chest trauma, systemic lupus erythmatosis, carcinoid heart disease, postpartum heart disease, Chagas' disease, beri-beri, amyloidosis, infectious myocarditis, endomyocardial fibrosis, sarcoidosis, endomyocardial biopsy, diverticulum of the left ventricle and Loffler's endocarditis (Nixon 1983). Diagnosis of intracardiac thrombus is further influenced by changes in imaging techniques and management strategies for different cardiac problems (Nixon 1983). As part of this thesis, I shall be concentrating on the thrombogenic tendency (as demonstrated in chapter 8) in patients with left ventricular dysfunction secondary to ischaemic heart disease, and the role of anticoagulation therapy.

\section{The size of the problem}

Thromboembolism is a frequent cause of death in patients with congestive heart failure, occurring in up to $30 \%$ of patients (Griffith et al 1952). Despite this, anticoagulant therapy has not been widely used as an adjuvant to routine therapy in patients with left ventricular dysfunction and left ventricular aneurysms. The reasons for these are uncertain, as discussed in chapter 8. However, the frequency of left ventricular thrombi in aneurysms seen at postmortem can range between 14 to $68 \%$ which is consistent with aneurysmectomy findings (50 to $95 \%$ ) (Nixon 1983). The reported incidences of systemic embolism, in patients with left ventricular aneurysm, demonstrates however a wide range, from 0 to $52 \%$ (Lapeyre et al 1985, Mullen et al 1977, Nixon 1983, Schlichter et al 1954).

There are few data supporting routine use of oral anticoagulant therapy in patients with left ventricular aneurysm. In one study (390 patients) there was a significant reduction in the incidence of thromboembolism in patients with chronic heart failure, especially in those with coexisting rheumatic heart disease (Griffith et al 1952). Warfarin therapy has also been associated with higher resolution of thrombus at echocardiography when compared to patients on no therapy ( $59 \%$ vs $29 \%$ respectively) (Stratton et al 1988). In a study of patients with congestive heart failure, the use of anticoagulant therapy reduced mortality by 5.8 to $8 \%$, with a 6 to $9 \%$ reduction in the incidence of thromboemboli (Griffith et al 1952). Importantly, cardiac arrhythmia, a past history of congestive cardiac failure or a past 
history of thromboemboli did not influence the outcome in these patients (Griffith et al 1952). In addition, anticoagulant therapy also reduces the risk of left ventricular thrombus and embolisation following acute myocardial infarction (Weinreich et al 1984). Many authorities therefore recommend prophylactic anticoagulation of large anterior myocardial infarctions.

Anticoagulant therapy may therefore provide protection against embolic events, but in contrast to the results discussed earlier, an echocardiographic study has demonstrated that the presence of thrombi at six months follow-up was the same regardless of anticoagulant treatment (Weinreich et al 1984). In addition, two surgical studies showed no apparent effect of anticoagulation on the prevalence of mural thrombus identified at aneurysmectomy (Reeder et al 1981, Simpson et al 1980). However, the duration of therapy correlates inversely with the presence of thrombus, and this may account for the discrepancy of these findings (Reeder et al 1981).

As discussed in chapter 8 , echocardiography is the method most commonly used for diagnosing left ventricular thrombi. Although up to $15 \%$ of studies are technically inadequate and cannot exclude the presence of thrombus, echocardiography may still be helpful in the stratification of patients into high and low risk for the development of mural thrombi and embolization. Similarly to patients with atrial fibrillation, the availability of a suitable plasma marker of ongoing thrombogenesis would also help in the risk stratification of such patients, especially those with intracardiac thrombi too small to be visualised by transthoracic echocardiography. 
10.2 STUDIES OF PLASMA FIBRINOGEN AND D-DIMER IN PATIENTS WITH CHRONIC ATRIAL FIBRILLATION, LEFT VENTRICULAR DYSFUNCTION AND LEFT VENTRICULAR ANEURYSMS: THE EFFECTS OF INTRODUCING ANTICOAGULANT THERAPY

Introduction

Five large studies have now established the role of oral anticoagulation therapy as prophylaxis against thromboembolism in patients with chronic nonrheumatic atrial fibrillation (Lowe 1992). In chapter 3 of this thesis I have demonstrated that patients with chronic atrial fibrillation on no antithrombotic therapy had significant abnormalities of plasma fibrinogen and fibrin D-dimer levels. The latter marker was however significantly lower in patients who were established on warfarin when compared to patients on no antithrombotic therapy. Chapter 8 of this thesis demonstrated similar findings for patients with left ventricular aneurysms, in which those established on warfarin therapy also had significantly lower plasma fibrin D-dimer levels when compared to patients who were not on warfarin. As plasma D-dimer is a marker of intravascular thrombus formation, my results are consistent therefore with the beneficial effects of warfarin in reducing thrombus formation. The effect of the introduction of warfarin therapy upon these plasma prothrombotic indices has not been previously investigated. If significant change does occur with warfarin, measurement of these markers may provide further information upon the action of warfarin in thromboembolic prophylaxis, the possible roles for these markers in identifying 'high risk' patients and monitoring the effectiveness of warfarin in reducing intravascular thrombogenesis.

The aim of this study therefore was to investigate the effects of introducing anticoagulant therapy in patients with atrial fibrillation and left ventricular aneurysms on markers of increased thrombogenesis as measured by plasma fibrinogen and fibrin D-dimer. 
Methods

A prospective study of all patients with atrial fibrillation or left ventricular aneurysms undergoing prophylactic anticoagulation was undertaken. The oral anticoagulation used was warfarin in all cases.

Prior to anticoagulation, all patients underwent echocardiography, primarily to assess for the presence of intracardiac thrombus. All patients were managed on an outpatient basis. At their initial visit, clinical examination and echocardiography was performed and baseline blood samples taken. Patients subsequently attended on Day 3, Day 5, Day 7 and 2 months following the introduction of warfarin for an INR (International Normalised Ratio) check to ensure satisfactory anticoagulation response.

Blood samples were also taken atraumatically at each visit and placed in citrate tubes (trisodium citrate $0.11 \mathrm{M}, 9: 1 \mathrm{v} \cdot \mathrm{v}$ ) for assay of plasma fibrinogen and fibrin D-dimer. The plasma was immediately separated and frozen at $-40^{\circ} \mathrm{C}$. Plasma fibrinogen and D-dimer antigen levels were measured in all samples, as described in previous chapters.

All the values of the rheological measurements were expressed as median \pm interquartile range (IQR). Initial (prewarfarin) levels of plasma fibrinogen and D-dimer were compared to normal population 'values', derived from 158 subjects in a healthy population survey, the 2nd North Glasgow MONICA study (as in previous chapters). Statistical comparison of sequential results was performed using the two-sample paired Wilcoxon test for non-parametric distributions. Other comparison of discontinuous results was performed using the Mann-Whitney test for non-parametric distributions and the $95 \%$ confidence intervals (c.i.) calculated. Correlations between the INR and prothrombotic markers were performed using Spearman's Rank correlation method. A probability of $\leq 0.05$ was considered statistically significant.

Results

A total of 30 patients were studied: 20 with chronic atrial fibrillation (13 male, 6 female; mean age 63.9 years, age range $32-74$ years, s.e.m. 2.35 ) whilst 10 had left ventricular dysfunction with aneurysm formation (mean age 52.5 years, age range 28-66 years, s.e.m. 4.33). Patient characteristics are summarised in Table 10.2. 
In patients with chronic atrial fibrillation, there was a significant elevation of initial (prewarfarin) levels of plasma fibrinogen (median difference $0.38 \mathrm{~g} / \mathrm{l} ; 95 \% \mathrm{C.I} \quad 0.10$ to 0.71, Mann Whitney $\mathrm{p}=0.009$ ) and fibrin D-dimer (median difference $96 \mathrm{ng} / \mathrm{ml} ; 95 \% \mathrm{C.I} .63$ to $134, p<0.0001$ ) when compared to population controls in sinus rhythm. In addition, there was a significant reduction in median plasma fibrin D-dimer levels at 2 months following the introduction of warfarin (181 ng/ml vs $80 \mathrm{ng} / \mathrm{ml}$; paired Wilcoxon test, $p<0.001$ ) (Table 10.3, Figure 10.1). Sequential plasma D-dimer results were also available for 16 patients at Days 3, 5 and 7 following the introduction of warfarin. There was a significant reduction in median plasma D-dimer at Day 3 following warfarin (162 ng/ml vs $136 \mathrm{ng} / \mathrm{ml}$; paired Wilcoxon test, $p=0.02$ ) when compared to prewarfarin levels. There were however no significant differences in median plasma D-dimer at Day $5(173 \mathrm{ng} / \mathrm{ml} ; \mathrm{p}=0.75)$ and Day $7(130 \mathrm{ng} / \mathrm{ml} ; \mathrm{p}=0.07)$ following the introduction of warfarin therapy. A median reduction of plasma fibrin D-dimer by $41 \%$ (95\% c.i. $32 \%$ to $64 \%)$ following the 2 months of anticoagulant therapy was therefore achieved. There were no significant changes in plasma fibrinogen levels at any of the sampling points following the introduction of warfarin therapy. (Table 10.4, Figure 10.1)

In patients with sinus rhythm, ischaemic heart disease and left ventricular aneurysms $(n=10)$, there was a significant elevation of initial (prewarfarin) levels of plasma fibrinogen (median difference $0.82 \mathrm{~g} /$; $95 \%$ C.I. 0.4 to $1.22, \mathrm{p}=0.0005$ ) and fibrin D-dimer (median difference $50 \mathrm{ng} / \mathrm{ml} ; 95 \%$ C.I. 20 to $104, \mathrm{p}=0.003$ ) when compared to population controls in sinus rhythm. There was a significant reduction in median plasma fibrin D-dimer levels at 2 months following the introduction of warfarin $(148 \mathrm{ng} / \mathrm{ml} \mathrm{vs} 61 \mathrm{ng} / \mathrm{ml}$; paired Wilcoxon test, $p=0.01$ ), although there were no significant differences in median plasma $D$ dimer at Day $3(128 \mathrm{ng} / \mathrm{ml} ; \mathrm{p}=0.47)$, Day $5(136 \mathrm{ng} / \mathrm{ml} ; \mathrm{p}=0.92)$ or Day $7(113 \mathrm{ng} / \mathrm{ml} ; \mathrm{p}=0.76)$ following the introduction of warfarin therapy when compared to prewarfarin levels (Table 10.3, Figure 10.2). In these patients, there was therefore a median reduction of plasma fibrin D-dimer by $58 \%$ (95\% c.i. $29 \%$ to $77 \%$ ) following 2 months of anticoagulation. There were again no significant differences in plasma fibrinogen levels in these patients following the introduction of warfarin therapy (Table 10.4, Figure 10.2). 
In the population controls, the upper limit of the interquartile range (IQR) [the 75th percentile] for plasma D-dimer was $103 \mathrm{ng} / \mathrm{ml}$ (Table 10.3). If we consider all the 30 patients studied and only selected those with a pre-warfarin plasma fibrin D-dimer level of $>100 \mathrm{ng} / \mathrm{ml}$ [an approximation of the 75 th percentile value for plasma D-dimer in the population] (23 patients; mean age 61.2 years, s.e.m. 2.79 ), the reduction of median plasma D-dimer levels following 2 months of warfarin therapy was highly significant $(200 \mathrm{ng} / \mathrm{ml}$ vs 68 $\mathrm{ng} / \mathrm{ml}$; paired Wilcoxon test, $\mathrm{p}<0.0001)$ with a median reduction of $49 \%$ (95 \% c.i. $36 \%$ to 72\%). There were no significant differences in median plasma D-dimer at Day 3 (145 ng/ml; $\mathrm{p}=0.27)$ or Day $5(175 \mathrm{ng} / \mathrm{ml} ; \mathrm{p}=0.59)$ following the introduction of warfarin therapy. At Day 7 , however, there was also a significant reduction in plasma D-dimer (200 vs $150 \mathrm{ng} / \mathrm{ml} ; \mathrm{p}=0.02)$. In this group, there were no significant differences in plasma fibrinogen levels at any of the sampling points (when compared to initial values) following the introduction of warfarin.

There was no significant correlation between plasma fibrinogen or fibrin Ddimer and INR levels at any of the sampling points in any of the groups studied (Table 10.5).

\section{Discussion}

This study demonstrates that plasma fibrinogen does not alter significantly following the introduction of warfarin therapy. As epidemiological studies have demonstrated that an elevated plasma fibrinogen level is a predictor of cardiovascular events, patients with chronic atrial fibrillation and left ventricular aneurysm demonstrate underlying haemorheological abnormalities and may continue to be at some cardiovascular risk despite being anticoagulated. This risk (and whether measurement of plasma fibrinogen is predictive of future cardiovascular events) remains to be demonstrated prospectively for patients with atrial fibrillation. Warfarin therapy is not known to influence any underlying pathological process and plasma fibrinogen levels would not have been expected to change after the introduction of warfarin therapy, as demonstrated in this study.

In contrast, there is a sequential reduction of plasma fibrin D-dimer following the introduction of warfarin therapy. This reduction was significant at 2 months or more following the introduction of warfarin. In addition, if we select patients with a high initial plasma 
fibrin D-dimer $(>100 \mathrm{ng} / \mathrm{ml})$, the reduction is significant at Day $7(p=0.02)$ and is highly significant $(p<0.0001)$ at two months, with a median reduction of $49 \%$. If warfarin effectively prevented intravascular fibrin turnover and thrombus formation, then plasma D-dimer levels would be expected to be much lowered in patients following the introduction of warfarin, and this effect of warfarin is clearly demonstrated in my study. The lack of correlation between plasma fibrin D-dimer and INR value suggests that two distinctly separate processes in thrombogenesis are being quantified: the INR would be assessing clotting factor activity (Factors II, VII, IX and X, which are vitamin K dependent factors) in the formation of clot, whilst the measurement of plasma fibrin D-dimer is an indirect quantification of fibrin turnover, which is the step that follows fibrin deposition in thrombus formation. In addition, quantification of INR (or prothrombin time) may not be directly related to the efficacy of warfarin in reducing thrombogenesis. This is demonstrated in a randomised, prospective trial in which the risk of thrombosis associated with a long-term central venous catheter was reduced by $65 \%$ in patients receiving only $1 \mathrm{mg} /$ day of warfarin, with no measurable change in coagulation values (Bern et al 1990). At this ultra-low-dose of warfarin, the in-vivo function of vitamin $K$ coagulation factors may be affected in ways not detectable by (ex-vivo) functional assays such as the INR or prothrombin time (Bern et al 1990). A reduction in fibrin turnover may be a mechanism by which ultra-low-dose warfarin exerts its benefits but this remains to be demonstrated.

My results are also consistent with the findings of the five large-scale clinical trials of anticoagulation therapy in atrial fibrillation (discussed in section 10.1). These studies show consistently that warfarin therapy reduces the risk of stroke and thromboembolism by approximately two-thirds (Lowe 1992). As increased plasma fibrin D-dimer levels suggest ongoing intravascular fibrin turnover and thrombus formation, patients with chronic atrial fibrillation and left ventricular dysfunction on no antithrombotic therapy are at increased risk of thrombogenesis. As anticoagulation therapy is itself not without risk, clinicians contemplating antithrombotic therapy for prophylaxis against stroke and thromboembolic events in patients with atrial fibrillation or left ventricular aneurysm have to balance the benefit of risk reduction against the risks of potentiating haemorrhage with warfarin therapy (Lowe 1992). The risk of 
any bleeding with the use of anticoagulation therapy ranges from $11 \%$ to $40 \%$ (Levine et al 1990). There is, of course, a potentially higher risk of bleeding with a greater level anticoagulation (for example, INR 2.5) when compared to patients with 'low level' anticoagulation (for example, INR 1.5) (Levine et al 1990, Saour et al 1990). Nevertheless, in a study of patients with prosthetic heart valves, no difference in the prophylactic effect against thromboembolism was detected between the two groups (Saour et al 1990). Similar conclusions were obtained in another study of patients with atrial fibrillation who were anticoagulated, in which bleeding was most frequent in patients with unstable anticoagulation control and those with a previous history of thromboembolism (Lundström and Rydén 1989). Bleeding complications may, however, occur frequently, even when the INR (or Thrombotest) is within the therapeutic range (Levine et al 1990, Lundström and Rydén 1989). In summary, although warfarin will prevent stroke and other thromboembolic events in $3.5 \%$ of treated patients per year, with the risk of important bleeding in $0.5 \%$ per year, there is (at present) no easy way to stratify patients for baseline risk and potential benefit (Ramsay 1993). My study suggests that the measurement of plasma fibrin D-dimer may identify a subgroup of patients at high risk of thrombogenesis (e.g. plasma D-dimer $>100 \mathrm{ng} / \mathrm{ml}$ ) whom would particularly benefit from the introduction of warfarin therapy. It may also identify those with the highest levels of intravascular clotting (that is, those with the highest plasma D-dimer levels) whom are theoretically at highest thromboembolic risk and these patients could possibly therefore be selected for more intense (or 'high level') anticoagulation. This would, however, need to be studied in a large scale prospective study to determine whether or not plasma levels of prothrombotic markers are predictive of future thromboembolic events.

The ability to select out patients at high risk of thrombogenesis may be particularly relevant in patients with heart failure. This is particularly pertinent as a recent survey of British cardiologists indicated that anticoagulant therapy would not be initiated in patients with heart failure due to dilated cardiomyopathy or ischaemic heart disease who remained in sinus rhythm (Cleland et al 1993). Reasons cited for not prescribing anticoagulant therapy included the fear of complications (54\%), inconvenience (17\%), lack of importance of thromboemboli (13\%) and lack of manpower resources (9\%) (Cleland et al 
1993). The presence of heart failure or ventricular impaiment is known however to be additive to the risk of thromboembolism in patients with atrial fibrillation (Stroke Prevention in Atrial Fibrillation Investigators 1992a, Stroke Prevention in Atrial Fibrillation Investigators 1992b). High risk patients with heart failure may be potentially identified therefore by the measurement of plasma fibrinogen and fibrin D-dimer, allowing the identification of those with a high prothrombotic state whom would particularly benefit from anticoagulant therapy.

This study is the first demonstration that the introduction of warfarin therapy in patients with atrial fibrillation and left ventricular aneurysms significantly reduces a laboratory index of thrombogenesis and fibrin turnover (that is, plasma fibrin D-dimer). The reduction of these processes by warfarin is consistent with the beneficial effect of warfarin therapy in reducing thromboembolic risk. It additionally suggests that measurement of plasma fibrin Ddimer may be useful as a marker of ongoing intravascular fibrin turnover and thrombogenesis, allowing further identification of those patients at high risk of thrombo-embolism. This marker may aid in decision-making when introduction of warfarin therapy is being considered. 
TABLE 10.1

RANDOMISED TRIALS OF ANTITHROMBOTIC PROPHYLAXIS IN ATRIAL FIBRILLATION

\begin{tabular}{|c|c|c|c|c|c|}
\hline $\begin{array}{c}\text { DESIGN } \\
\text { (n) }\end{array}$ & $\begin{array}{l}\text { SPAF } \\
1330\end{array}$ & $\begin{array}{c}\text { AFASAK } \\
1007\end{array}$ & $\begin{array}{c}\text { BAAT AF } \\
420\end{array}$ & $\begin{array}{c}\text { CAFA } \\
383\end{array}$ & $\begin{array}{c}\text { SPINAF } \\
538\end{array}$ \\
\hline $\begin{array}{c}\text { mean age } \\
\text { (years) }\end{array}$ & 67 & 74 & 68 & 68 & 67 \\
\hline $\begin{array}{l}\text { mean follow-up } \\
\text { (years) }\end{array}$ & 1.3 & 1.2 & 2.2 & 1.3 & 1.7 \\
\hline $\begin{array}{c}\text { male } \\
\%\end{array}$ & 71 & 54 & 72 & 75 & 100 \\
\hline $\begin{array}{l}\text { INR range } \\
\text { PTR range }\end{array}$ & $1.3-1.8$ & $\begin{array}{c}2.8-4.2 \\
\ldots\end{array}$ & $1.2-1.5$ & $\begin{array}{c}2.0-3.0 \\
, \ldots\end{array}$ & $1.2-1.5$ \\
\hline $\begin{array}{l}\text { Aspirin dose } \\
\text { (mg/day) }\end{array}$ & 325 & 75 & $\ldots$ & $\ldots$ & $\ldots$ \\
\hline $\begin{array}{c}\text { Ischaemic stroke } \\
\text { (\%) } \\
\text { placebo group } \\
\text { warfarin group } \\
\text { aspirin group }\end{array}$ & $\begin{array}{l}6.3 \\
2.3 \\
3.6\end{array}$ & $\begin{array}{l}5.5 \\
2.2 \\
4.7\end{array}$ & $\begin{array}{c}0.4 \\
\ldots\end{array}$ & $\begin{array}{c}4.6 \\
3.0 \\
\ldots\end{array}$ & $\begin{array}{l}3.9 \\
1.0 \\
\ldots\end{array}$ \\
\hline $\begin{array}{l}\text { Major bleeding } \\
\text { (\% per year) } \\
\text { warfarin group }\end{array}$ & 1.5 & 0.5 & 0.8 & 2.1 & 1.3 \\
\hline
\end{tabular}

[AFASAK=Copenhagen Atrial Fibrillation, Aspirin, Anticoagulation Study; SPAF=Stroke Prevention in Atrial Fibrillation Study; BAATAF=Boston Area Anticoagulation Trial for Atrial Fibrillation; CAFA=Canadian Atrial Fibrillation AnticoagulationStudy; SPINAF=Veterans Affairs StrokePrevention in Nonrheumatic Atrial Fibrillation study] 
TABLE 10.2

\section{ANTICOAGULANT THERAPY -PATIENT CHARACTERISTICS}

\author{
GROUP I \\ atrial fibrillation \\ GROUP II \\ left ventricular aneurysm
}

20

63.4

(2.4)

$14: 6$

7

41.2

(35.9-43.5)
10

52.5

(4.3)

$8: 2$

2

39.9

(38.9-41.0) mean (s.e.m.)

body mass

index

mean $(\mathrm{kg} / \mathrm{m} 2)$ 
TABLE 10.3

\section{PLASMA D-DIMER LEVELS \\ AND ANTICOAGULATION}

\begin{tabular}{|c|c|c|c|c|c|}
\hline $\begin{array}{l}\text { PATIENT } \\
\text { GROUPS }\end{array}$ & Pre-warfarin & Day 3 & Day 5 & Day 7 & 2 months \\
\hline $\begin{array}{c}\text { ATRIAL } \\
\text { FIBRILLATION } \\
(n=20)\end{array}$ & $\begin{array}{c}181 \\
(111-235)\end{array}$ & $\begin{array}{c}136^{\star} \\
(90-200)\end{array}$ & $\begin{array}{c}173 \\
(125-244)\end{array}$ & $\begin{array}{c}130 \\
(92-209)\end{array}$ & $\begin{array}{c}80^{\star} \\
(61-137)\end{array}$ \\
\hline $\begin{array}{l}\text { LV ANEURYSM } \\
\qquad(n=10)\end{array}$ & $\begin{array}{c}115 \\
(96-223)\end{array}$ & $\begin{array}{c}128 \\
(108-259)\end{array}$ & $\begin{array}{c}136 \\
(91-227)\end{array}$ & $\begin{array}{c}113 \\
(94-172)\end{array}$ & $\begin{array}{c}61^{\star} \\
(54-67)\end{array}$ \\
\hline $\begin{array}{c}\text { Whole group } \\
\text { with initial plasma } \\
\text { D-DIMER } \\
>100 \mathrm{ng} / \mathrm{ml}\end{array}$ & $\begin{array}{c}200 \\
(136-271)\end{array}$ & $\begin{array}{c}145 \\
(119-242)\end{array}$ & $\begin{array}{c}175 \\
(126-230)\end{array}$ & $\begin{array}{c}150^{\star} \\
(95-200)\end{array}$ & $\begin{array}{c}68^{\star *} \\
(57-98)\end{array}$ \\
\hline
\end{tabular}

Median plasma D-Dimer values for population controls $(n=158): 76 \mathrm{mg} / \mathrm{dl}$ (IQR 53-103) ( ${ }^{\star} p<0.05 ;{ }^{\star \star} p<0.0001$ when compared to pre-warfarin levels) 
TABLE 10.4

\section{PLASMA FIBRINOGEN LEVELS AND ANTICOAGULATION}

PLASMA FIBRINOGEN median (IQR) $g / l$

\begin{tabular}{|c|c|c|c|c|c|}
\hline $\begin{array}{l}\text { PATIENT } \\
\text { GROUPS }\end{array}$ & Pre-warfarin & Day 3 & Day 5 & Day 7 & 2 months \\
\hline $\begin{array}{c}\text { ATRIAL } \\
\text { FIBRILLATION } \\
(n=30)\end{array}$ & $\begin{array}{c}3.0 \\
(2.5-3.42)\end{array}$ & $\begin{array}{c}3.17 \\
(2.69-3.66)\end{array}$ & $\begin{array}{c}3.12 \\
(2.52-3.54)\end{array}$ & $\begin{array}{c}2.92 \\
(2.38-3.66)\end{array}$ & $\begin{array}{c}3.30 \\
(2.77-4.02)\end{array}$ \\
\hline $\begin{array}{l}\text { LV ANEURYSM } \\
\qquad(n=10)\end{array}$ & $\begin{array}{c}3.37 \\
(3.01-3.84)\end{array}$ & $\begin{array}{c}3.22 \\
(2.57-3.82)\end{array}$ & $\begin{array}{c}3.28 \\
(2.71-3.71)\end{array}$ & $\begin{array}{c}3.71 \\
(2.81-3.45)\end{array}$ & $\begin{array}{c}3.24 \\
(3.02-3.15)\end{array}$ \\
\hline $\begin{array}{c}\text { Whole group } \\
\text { with initial plasma } \\
\text { D-DIMER } \\
>100 \mathrm{ng} / \mathrm{ml} \\
(n=23)\end{array}$ & $\begin{array}{c}3.09 \\
(2.53-4.03)\end{array}$ & $\begin{array}{c}3.35 \\
(2.56-3.73)\end{array}$ & $\begin{array}{c}3.18 \\
(2.61-3.79)\end{array}$ & $\begin{array}{c}3.05 \\
(2.58-3.85)\end{array}$ & $\begin{array}{c}3.10 \\
(2.87-3.78)\end{array}$ \\
\hline
\end{tabular}

Median plasma Fibrinogen values for population controls $(n=158): 2.6 \mathrm{~g} / 1$ (IQR 2.24-2.99) $(p=N S)$ 
TABLE 10.5

\section{SPEARMAN CORRELATIONS \\ BETWEEN PLASMA D-DIMER LEVELS \\ AND ANTICOAGULATION}

\begin{tabular}{|c|c|c|c|c|c|c|c|c|}
\hline \multirow{2}{*}{$\begin{array}{l}\text { D-Dimer } \\
\text { vs INR }\end{array}$} & \multicolumn{2}{|c|}{ DAY 3} & \multicolumn{2}{|c|}{ DAY 5} & \multicolumn{2}{|c|}{ DAY 7} & \multicolumn{2}{|c|}{2 months } \\
\hline & $\mathbf{r}$ & p & $\mathbf{r}$ & $p$ & $r$ & $p$ & $\mathbf{r}$ & $\mathbf{p}$ \\
\hline $\begin{array}{c}\text { ATRIAL } \\
\text { FIBRILLATION } \\
\text { group }(n=30)\end{array}$ & 0.33 & 0.216 & 0.065 & 0.81 & 0.22 & 0.57 & 0.24 & 0.65 \\
\hline $\begin{array}{l}\text { LV ANEURYSM } \\
\text { group }(n=10)\end{array}$ & -0.16 & 0.68 & -0.35 & 0.64 & -0.24 & 0.57 & -0.37 & 0.29 \\
\hline
\end{tabular}

$p=N S$

(INR = International Normalised Ratio) 


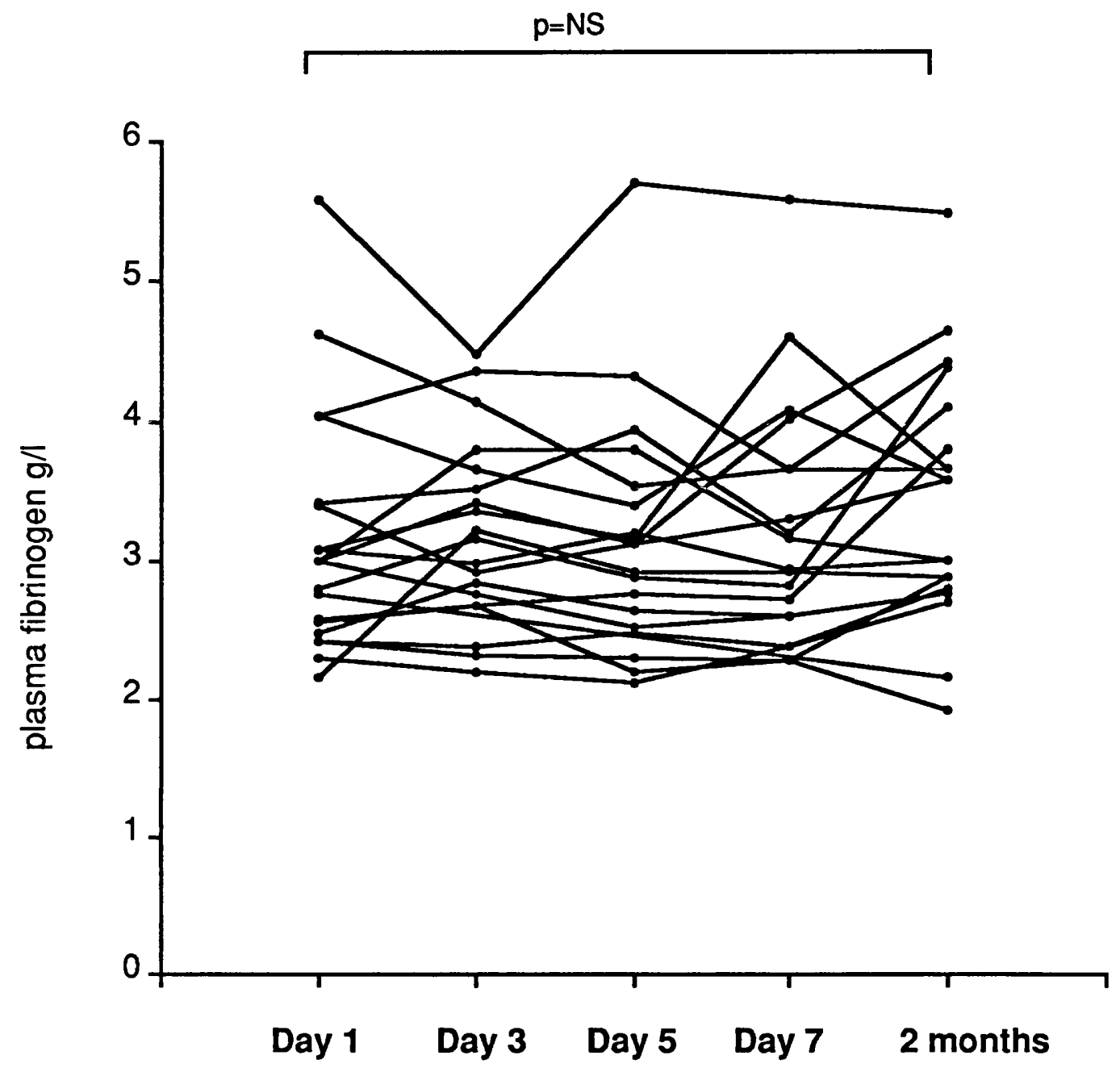

FIGURE 10.1 (a)

ANTICOAGULATION AND PLASMA FIBRINOGEN IN ATRIAL FIBRILLATION 


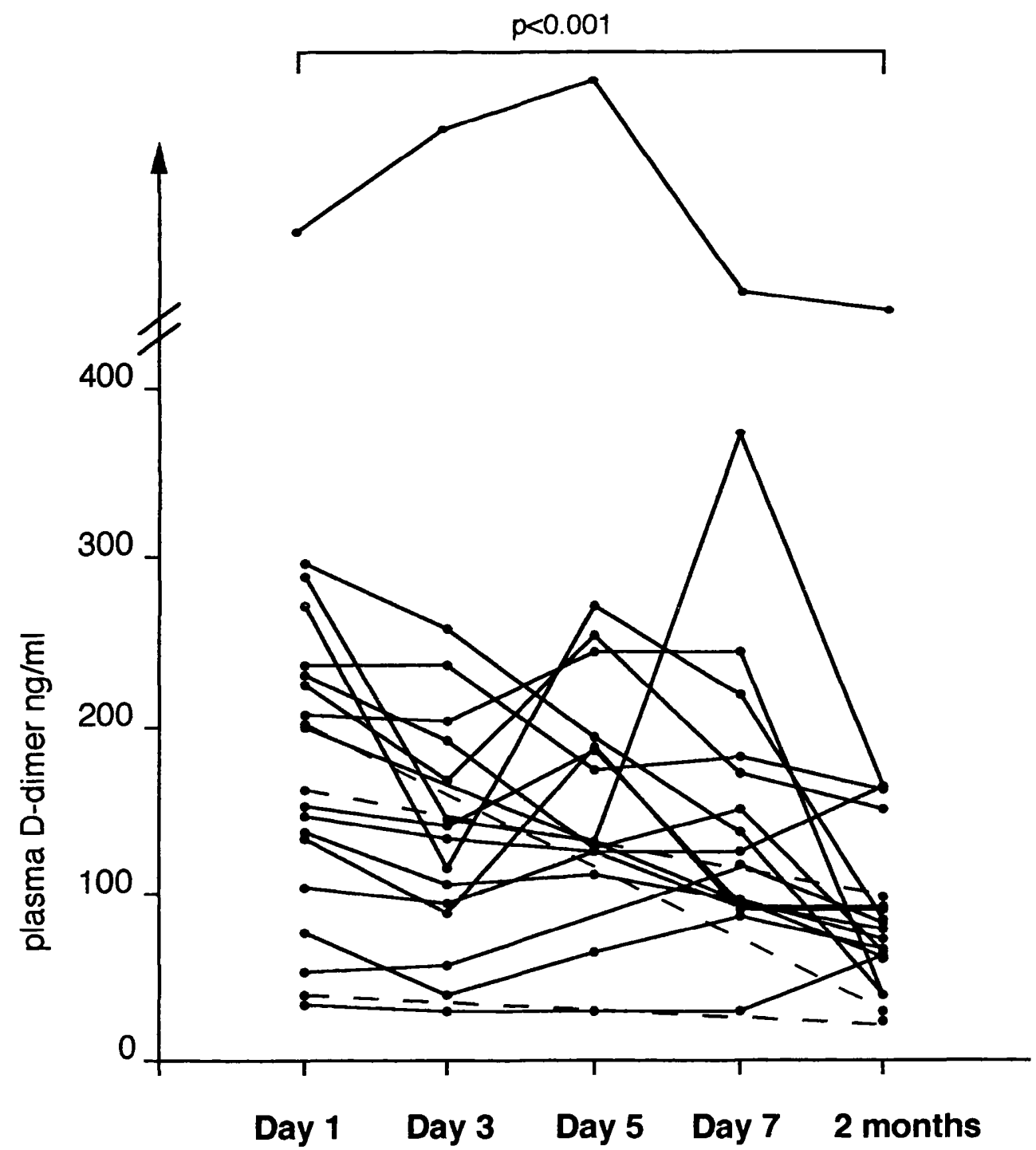

FIGURE 10.1 (b)

ANTICOAGULATION AND PLASMA D-DIMER IN ATRIAL FIBRILLATION 


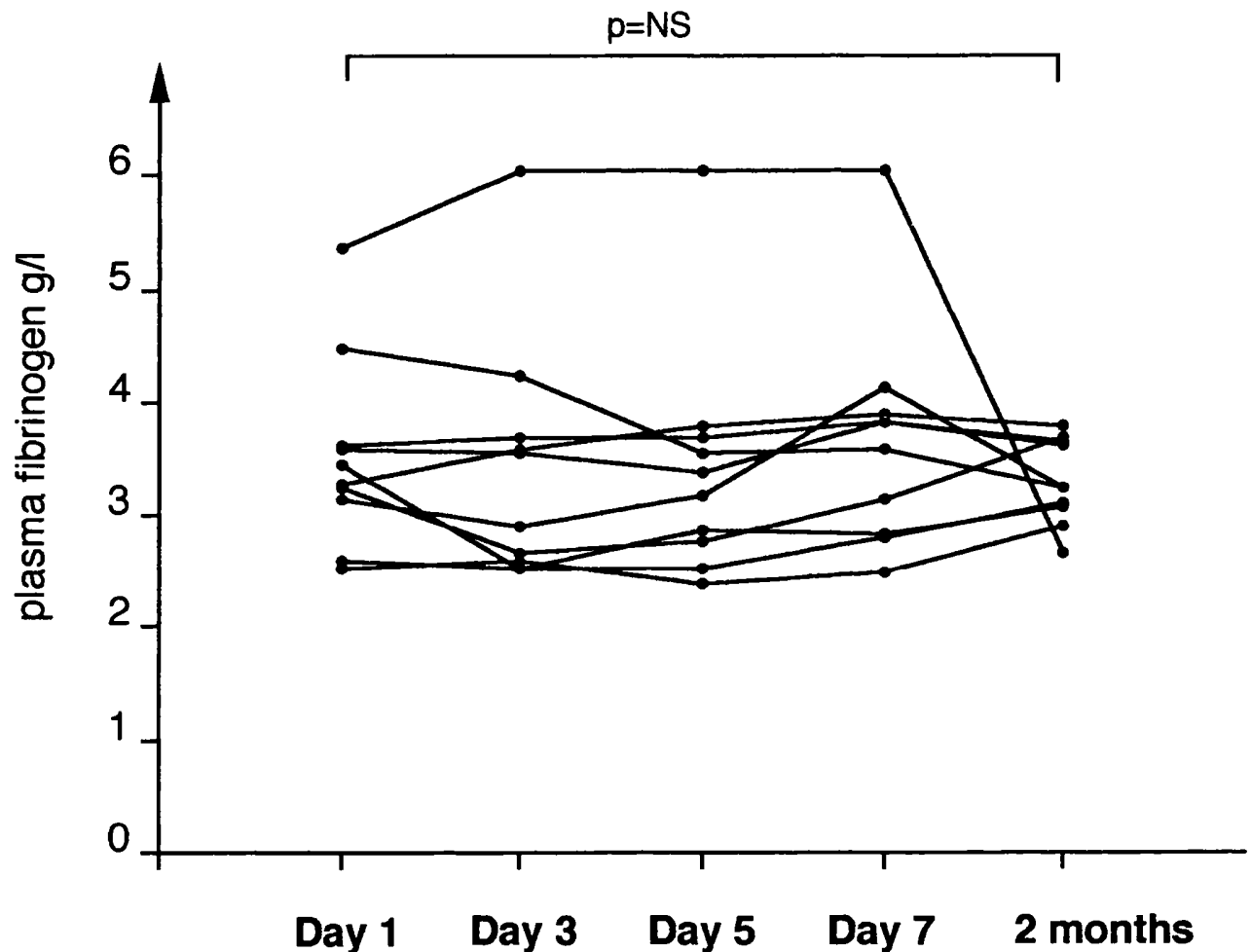

FIGURE 10.2 (a) ANTICOAGULATION AND PLASMA FIBRINOGEN IN LEFT VENTRICULAR ANEURYSMS 


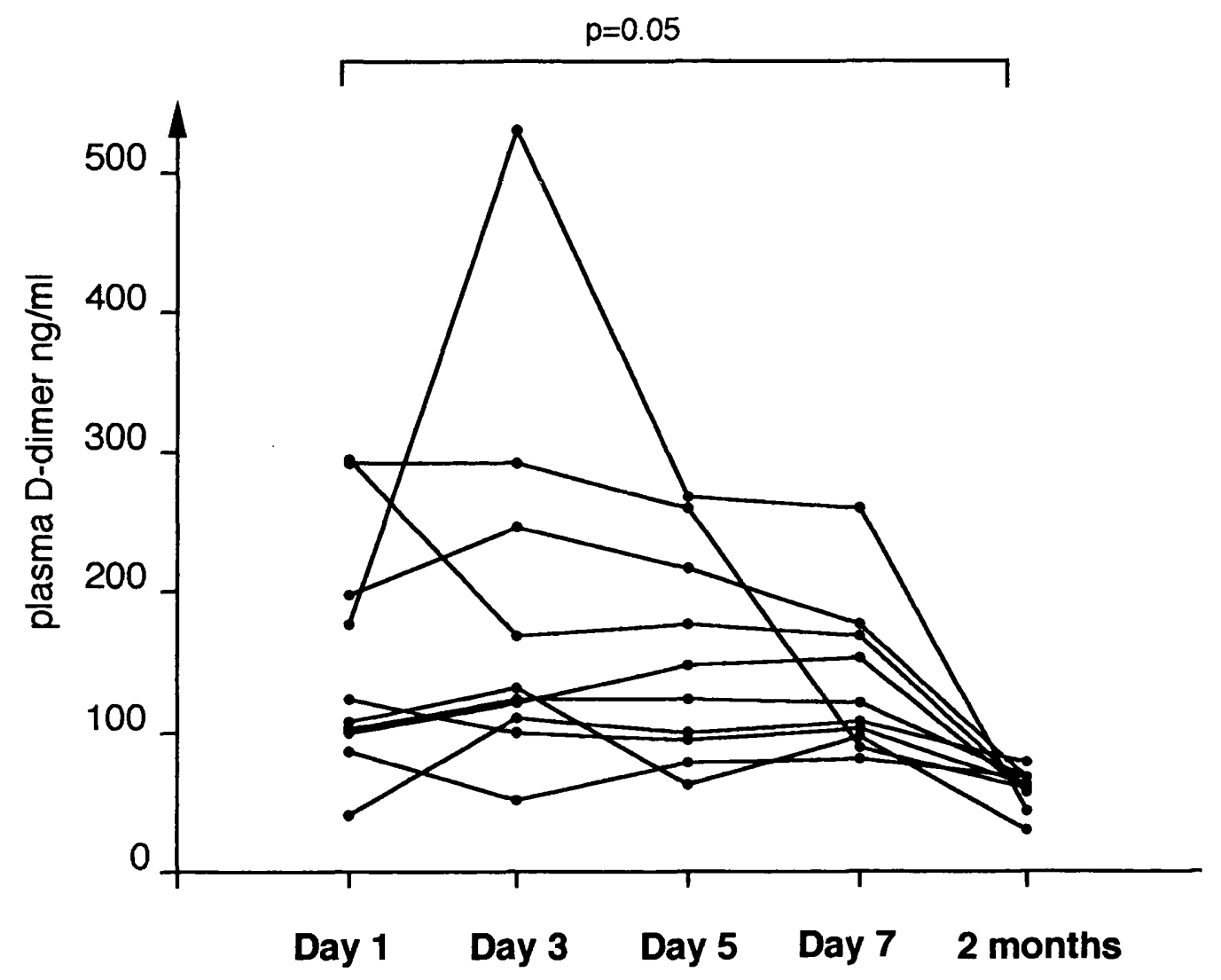

FIGURE 10.2 (b) ANTICOAGULATION AND PLASMA D-DIMER IN LEFT VENTRICULAR ANEURYSMS 
CHAPTER 11

LIPOPROTEIN(a)

AND

THROMBOGENESIS

IN

ATRIAL FIBRILLATION

AND

LEFT VENTRICULAR

DYSFUNCTION 


\subsection{LIPOPROTEIN (a) IN CHRONIC ATRIAL FIBRILLATION AND LEFT VENTRICULAR DYSFUNCTION}

Introduction

In earlier chapters of this thesis, I have demonstrated significant abnormalities of plasma fibrinogen, fibrin D-dimer and von Willebrand factor in patients with atrial fibrillation and left ventricular aneurysms, suggesting roles for fibrinogen, intravascular fibrin turnover and endothelial dysfunction in the process of thrombogenesis in these patients.

Lipoprotein (a), another serum factor, may also be associated with thrombosis (Rees 1991, Scott 1991). This is in part based on the structural similarity of apolipoprotein (a) (contained in lipoprotein (a)) to plasminogen. In view of this finding, lipoprotein (a) may theoretically have thrombolytic properties, but clinical evidence for this is still lacking. In contrast, lipoprotein (a) may competitively inhibit the binding (by competing with plasminogen for plasminogen receptors) of plasminogen to fibrin, thereby preventing the process of fibrinolysis (Loscalzo et al 1990, Rees 1991, Scott 1991) and thus creating a potential thrombogenic state. Theoretically, high levels of lipoprotein (a) may, therefore, significantly inhibit fibrinolysis and fibrin turnover. As plasma fibrin D-dimer is a measure of fibrin turnover, an inverse relationship between lipoprotein (a) levels and plasma fibrin D-dimer levels may exist. However, the contribution of lipoprotein (a) to the mechanisms of thrombogenesis (for example, clotting factor abnormalities, fibrin turnover, endothelial dysfunction) has not been defined.

Few epidemiological studies have specifically investigated the association between lipoprotein(a) levels and thromboembolic risk. However, there is evidence that lipoprotein (a) is an independent risk factor for myocardial infarction, being associated with atherosclerotic disease and coronary graft restenosis (Dahlen et al 1986, Hoff et al 1988, Rosengren et al 1990), and stroke (Pedro-Botet et al 1992, Woo et al 1991, Zenker et al 1986). In addition, it has been suggested that apolipoprotein (a) (contained in lipoprotein (a)) could offer some selective advantage in tissue or wound repair, as it may help deliver cholesterol by attaching (due to its fibrin-binding capability) to thrombus (Lawn 1992, Scanu 
1992). Although higher levels of lipoprotein (a) are seen in patients with ischaemic heart disease, relatively lower lipoprotein (a) levels in certain patients may, for example, contribute to poor tissue healing post-myocardial infarction. In patients with large myocardial infarctions, such poor healing may lead to aneurysm formation.

The aim of this chapter was to determine whether or not lipoprotein (a) levels are associated with thrombogenesis, by a study of the two major patient groups in which the highest intravascular thrombogenesis has been demonstrated: patients with chronic atrial fibrillation and left ventricular aneurysm. In addition, I also wished to determine whether or not there was a relationship between lipoprotein (a) and fibrin turnover (as assessed by plasma fibrin D-dimer) and an association with aneurysm formation.

\section{Patients and methods}

A prospective case-controlled study was undertaken of male and female outpatients with established chronic atrial fibrillation (Group A) and ischaemic heart disease and left ventricular dysfunction (Group B). Chronic atrial fibrillation was defined as per chapter 3, whilst left ventricular dysfunction and aneurysm formation were defined using methods described in chapter 8 . The relationship between lipoprotein (a) and left ventricular diastolic dysfunction was also determined using methods described in chapter 9 . Exclusion criteria for this study were similar to chapters 3,8 and 9 .

Blood samples were obtained from outpatients who were either fasting (if studied in the morning) or after a light breakfast (if studied in the afternoon) by careful venepuncture. In patients in Group A (chronic atrial fibrillation), the blood was placed in citrate tubes (trisodium citrate $0,11 \mathrm{M}, 9: 1 \mathrm{v}: \mathrm{v}$ ), the plasma was separated and frozen at $-40^{\circ} \mathrm{C}$. As patients in Group B (left ventricular dysfunction) were studied at a later period, a clotted sample was obtained, the serum separated, and frozen similarly. Plasma and serum samples were also obtained and frozen for a control group of 22 healthy volunteers of similar age $( \pm 5$ years) and sex. Assays for lipoprotein (a) were performed using two methods, based on the type of sample. Plasma lipoprotein (a) levels were assayed using a commercially available ELISA method (IMMUNOZYM LP(a) assay, IMMUNO, Vienna, Austria) whilst serum lipoprotein 
(a) was assayed using an immuno-turbidometic assay (INCSTAR Corp., Stillwater, Minnesota, USA). The coefficient of variation was $2-9 \%$ between assays for the immuno-turbidometic method (INCSTAR) and $11 \%$ for the ELISA method (IMMUNOZYM). Serum cholesterol and triglycerides were also determined in Group B patients using an enzymatic method (Olympus AU5223 Analyser, Olympus Corp., Japan). A comparison between lipoprotein (a) levels and corresponding plasma fibrinogen, fibrin D-dimer and von Willebrand factor, as described in earlier chapters, was also performed to assess the relationship, if any, with these factors.

All measured values of the serum and plasma factors were expressed as median \pm interquartile range (IQR). Statistical comparisons were performed using the KruskalWallis test and Mann-Whitney two sample rank test for non-parametric distributions, and the 95\% confidence intervals (c.i.) calculated for the point estimate of the difference between medians. Correlations between clinical variables and serum/plasma measurements were performed using Spearman's Rank correlation method. Analysis of the influence of systolic and diastolic dysfunction and their interaction on lipoprotein (a) was performed using ANOVA for unbalanced data (General Linear Model). A probability of $\leq 0.05$ was considered statistically significant. Reproducibility for measurement of lipoprotein (a) by the two methods was made using linear regression and the method described by Bland and Altman (1986).

Results

Fifty-seven patients were enrolled into Group A (chronic atrial fibrillation) and 105 patients into Group B (ischaemic heart disease). In Group A, there were 20 patients on no antithrombotic therapy, 25 on warfarin, and 12 on aspirin. In Group B, left ventricular systolic function was defined (as described in chapter 8) for 101 patients: 29 patients had normal left ventricular function (Group 1); 30 patients had left ventricular dysfunction (Group 2); 23 patients had left ventricular aneurysms and were not on warfarin (Group 3a); and 19 patients had left ventricular aneurysms and were taking warfarin therapy (Group 3b). Doppler echocardiography was performed to define diastolic dysfunction in 86 patients (in whom systolic function was previously categorised as in chapter 9), whom were not on warfarin therapy. Results for lipoprotein (a) were compared to 22 healthy volunteers of similar age and 
sex as a control group. Serum and plasma lipoprotein (a) levels were measured in controls to allow corresponding comparisons.

In Group A, there were no significant differences between plasma lipoprotein (a) levels in patients with chronic atrial fibrillation (irrespective of warfarin or aspirin therapy) when compared to healthy controls (Kruskal-Wallis $H=2.03$, d.f. $=3 ; p=0.565$ ) (Table 11.1, Figure 11.1). There were no significant correlations with corresponding levels of plasma fibrinogen, fibrin D-dimer or von Willebrand factor (see chapter 3). There was also no significant correlation with atrial size, left ventricular end-diastolic dimension and left ventricular function (as measured by fractional shortening).

In Group B, there were no significant differences in serum lipoprotein (a) between patients with leít ventricular aneurysm or left ventricular dysfunction when compared with patients having normal left ventricular function and with healthy controls. (Table 11.2, Figure 11.2). There was also no difference between lipoprotein (a) levels in patients on warfarin therapy (Group 3b) when compared with Group 3a (Table 11.2). No significant differences in lipoprotein (a) (Kruskal-Wallis $H=1.41$, d.f. $=4 ; p=0.842$ ), cholesterol (KruskalWallis $H=6.48$, d.f. $=4 ; p=0.167$ ) and triglycerides (Kruskal-Wallis $H-8.21$, d.f. $=4 ; p=0.085$ ) were demonstrable between the groups. There were no significant correlations between lipoprotein (a) levels and corresponding levels of plasma fibrinogen, fibrin D-dimer, von Willebrand factor, cholesterol or triglycerides in these patients (Table 11.2).

In the subgroups of Group B with normal systolic function and left ventricular systolic dysfunction, there were no significant differences in lipoprotein (a) levels in patients with or without diastolic dysfunction. In those with left ventricular aneurysms, however, plasma lipoprotein (a) was significantly elevated in those without diastolic dysfunction when compared to those with diastolic dysfunction (point estimate of difference between medians $33 \mathrm{mg} / \mathrm{ml}, 95 \%$ c.i. 11 to $49, \mathrm{p}=0.024$ ). Using ANOVA for unbalanced data (GLM or General Linear Model analysis), there was no significant effect of the presence of an aneurysm or systolic dysfunction and diastolic dysfunction (or the interaction between them) upon lipoprotein (a) levels. 
When the two lipoprotein (a) assay methods on the 22 control samples were compared the mean difference between them was $5.5 \mathrm{mg} / \mathrm{dl} \pm 21$ (2s.d.). The Pearson correlation for the two methods was however excellent $(r=0.968)$.

\section{Discussion}

Lipoprotein (a) is associated with atherogenesis and thrombogenesis (Rees 1991, Scott 1991). However, there is little information on whether or not it is increased in conditions of high thromboembolic risk. I have therefore investigated changes of lipoprotein (a) in two cardiac conditions in which 1 have previously demonstrated increased thrombogenesis; that is, chronic atrial fibrillation and left ventricular aneurysms. This study, however, demonstrates no significant differences in median plasma lipoprotein(a) levels in patients with chronic atrial fibrillation when compared to controls. There were also no significant differences in median serum lipoprotein (a) in patients with left ventricular aneurysms or left ventricular dysfunction when compared to patients with normal left ventricular function and controls. In addition, despite the association with increased thrombosis, there was no significant alteration of lipoprotein (a) levels in the patients established on warfarin therapy. It is conceivable therefore that the 'strong' genetic influence upon Lp(a) levels (Sandholzer et al 1992, Scott 1991) may explain these differences. For example, perhaps some $L p(a)$ phenotypes do not contribute to thrombogenesis and as a result, some patients with high $L p(a)$ may not be at risk of thrombogenesis. Some evidence for such a dissociation has already been provided by population comparisons. For example, black Americans have higher $L p(a)$ levels than whites, despite having lower rates of coronary artery disease (Guyton et al 1985, Scanu 1992). By a similar analogy, phenotypes associated with high $L p(a)$ levels but not with thrombogenic complications might be common in the population studied in this chapter.

Subgroup analysis of patients in Group B into those with and without diastolic dysfunction (by echocardiographic criteria, as defined in chapter 9) shows that $L(a)$ levels are higher in patients with left ventricular aneurysms without diastolic dysfunction when compared to similar patients with diastolic dysfunction. Two possible mechanisms may explain this 
finding. Firstly, patients with high $L p(a)$ levels may have the greatest atherogenic and thrombogenic risk, thus sustaining the largest myocardial infarctions, which lead to large aneurysms with major impairment of systolic function. Alternatively, patients with low Lp(a) levels may have poor tissue healing post-myocardial infarction, leading to aneurysm formation with marked fibrosis, reduced ventricular compliance and increased wall stiffness; these factors resulting in diastolic dysfunction. It should be noted however that the values of Lp(a) are widely spread, even in these subgroups.

Although I have used healthy volunteers as controls, the level of lipoprotein (a) [median $22 \mathrm{mg} / \mathrm{dl}$ by ELISA, $24 \mathrm{mg} / \mathrm{dl}$ by immunoturbidometric assay] appears higher than levels quoted in the large Framingham Offspring Study for patients free of cardiovascular disease (Jenner et al 1993). In this latter study, mean concentrations of $L p(a)$ were $14 \mathrm{mg} / \mathrm{dl}$ (s.d.17) in men and $15 \mathrm{mg} / \mathrm{dl}$ in women (s.d.17) (Jenner et al 1993). This difference may be a reflection of my small sample size of 'healthy' volunteers, as several subjects could have underlying coronary artery disease and a different prevalence of apolipoprotein (a) phenotypes (which were not studied). Larger studies, with local population-based healthy controls may be required therefore to define the 'normal' value of the local population.

It has been suggested that lipoprotein (a) may interfere with fibrinolysis through its competition with plasminogen for binding on fibrin and/or on endothelial cells. If significant interference with fibrinolysis occurs, high lipoprotein (a) levels may inhibit fibrinolysis sufficiently to reduce fibrin turnover, thereby reducing plasma levels of fibrin Ddimer. In my study, there was however no relationship between lipoprotein (a) and fibrin Ddimer demonstrable. This finding is consistent with clinical observations that raised lipoprotein (a) levels do not adversely affect coronary recanalization (demonstrated angiographically) with the use of thrombolytic or fibrinolytic agents (saruplase (Armstrong et al 1990) and alteplase (Tranchesi et al 1991)) in patients with myocardial infarction. My findings also complement the results of Garcia Frade et al (1991), in which no correlation was found between lipoprotein (a) and fibrinolytic parameters in a study of 34 patients with chronic angina and 12 with myocardial infarction. All these results are, however, in contrast to previous observations that plasma lipoprotein(a) can inhibit the fibrinolytic activity generated by streptokinase, although this 
thrombolytic agent was not used in the studies discussed earlier. For example, streptokinase may bind to lipoprotein (a), resulting in inhibition of streptokinase-mediated activation of human plasminogen to plasmin (Edelberg et al 1989, Karadi et al 1988). Differences between thrombolytic agents may therefore exist.

The PROCAM study demonstrated a weak association between lipoprotein (a) and fibrinogen levels (Heinrich et al 1991a), although this investigated considerably more patients than here. In my study, however, I was unable to demonstrate any significant association between fibrinogen and lipoprotein (a), but this may possibly be a reflection of smaller numbers and differences in the populations studied. However, if any major associations were present, it might have been expected that some evidence would have been found in this study. In addition, there were also no significant correlations between lipoprotein (a) and cholesterol, triglycerides, plasma fibrinogen, D-dimer or von Willebrand factor. This lack of any relationship is consistent with previous studies (Zenker et al 1986) and is most probably a reflection of the major genetic influence on lipoprotein (a) levels.

In conclusion, no significant abnormalities of lipoprotein (a) levels have been demonstrated in the two previously described groups of patients with the highest intravascular fibrin turnover (and therefore, thrombogenesis). This study suggests therefore that there may be no important interactions between lipoprotein (a) and thrombogenesis, and if any relationship does exist, it is probably weak. 
TABLE 11.1

\section{CHRONIC ATRIAL FIBRILLATION AND LIPOPROTEIN (a)}

(a) Lipoprotein (a) levels in atrial fibrillation groups:

$\begin{array}{lcccc} & \begin{array}{c}\text { CHRONIC } \\ \text { no therapy }\end{array} & \begin{array}{c}\text { ATRIAL } \\ \text { warfarin }\end{array} & \begin{array}{c}\text { FIBRILLATION } \\ \text { aspirin }\end{array} & \begin{array}{c}\text { CONTROLS } \\ \mathrm{n}\end{array} \\ & 20 & 25 & 12 & 23 \\ \text { oprotein } & 12 & & & \\ \text { (a) } & (5-31) & (5-29) & (2-23) & (4-46) \\ \text { median } & & & & \\ \text { mg/dl } & & & & \\ \text { (IQR) } & & & & \end{array}$

(b) Spearman correlations for Lipoprotein (a) in patients not on warfarin:

$\begin{array}{lcc}\text { Fibrinogen } & 0.064 & 0.73 \\ \text { D-dimer } & -0.09 & 0.65 \\ \text { von Willebrand factor } & -0.345 & 0.075 \\ \text { AGE } & -0.172 & 0.65 \\ \text { BODY MASS INDEX } & -0.11 & 0.554 \\ \text { LEFT ATRIAL dimension } & -0.049 & 0.788 \\ \text { LEFT ATRIAL volume } & -0.063 & 0.732 \\ \text { RIGHT ATRIAL volume } & 0.289 & 0.133 \\ \text { LV end diastolic dimension } & 0.193 & 0.325 \\ \text { LV fractional shortening } & -0.118 & 0.557\end{array}$


TABLE 11.2

\section{LEFT VENTRICULAR SYSTOLIC DYSFUNCTION} AND LIPOPROTEIN (a)

$\begin{array}{ccccc}\text { GROUP } 1 & \text { GROUP } 2 & \text { GROUP } 3 a & \text { GROUP 3b } & \text { HEALTHY } \\ \text { normal LV } & \text { LV } & \text { LV } & \text { LV } & \text { CONTROLS } \\ & \text { dysfunction } & \begin{array}{c}\text { aneurysm } \\ \text { no warfarin }\end{array} & \begin{array}{c}\text { aneurysm } \\ \text { warfarin }\end{array} \\ 29 & 30 & 23 & 19 & 21\end{array}$

lipoprotein

(a)

$\mathrm{mg} / \mathrm{dl}$

31

21

32

24

27

median

(13-60)

(12-46)

$(11-61)$

$(10-46)$

(IQR)

cholesterol

$\begin{array}{cccccc}\text { mmol/I } & 6.5 & 6.7 & 6.3 & 6.7 & 5.7 \\ \text { median } & (5.6-7.1) & (5.7-7.5) & (6.0-6.95) & (5.9-7.1) & (5.0-6.7) \\ \text { (IQR) } & & & & & \end{array}$

triglycerides

$\begin{array}{cccccc}\text { mmol/ } & 1.9 & 1.9 & 2.1 & 1.9 & 1.4 \\ \text { median } & (1.6-3.2) & (1.5-2.9) & (1.3-2.7) & (1.2-2.5) & (1.0-1.8) \\ \text { (IQR) } & & & & & \end{array}$

$p=N S$

(b) Spearman correlations for Lipoprotein (a):

\begin{tabular}{lcccccc} 
& GROUP & & GROUP & \multicolumn{3}{c}{ GROUP } \\
& $\mathbf{r}$ & $\mathbf{p}$ & $\mathbf{r}$ & $\mathbf{p}$ & $\mathbf{r}$ & $\mathbf{p}$ \\
fibrinogen & -0.194 & 0.304 & -0.203 & 0.289 & -0.061 & 0.785 \\
D-dimer & -0.06 & 0.76 & -0.32 & 0.091 & 0.007 & 0.973 \\
vWF & -0.03 & 0.87 & -0.082 & 0.686 & -0.336 & 0.122 \\
CHOLESTEROL & 0.177 & 0.639 & -0.25 & 0.183 & -0.077 & 0.73 \\
TRIGLYCERIDE & -0.119 & 0.545 & 0.086 & 0.657 & -0.22 & 0.323
\end{tabular}


TABLE 11.3

\section{LEFT VENTRICULAR DIASTOLIC DYSFUNCTION AND LIPOPROTEIN (a)}

\begin{tabular}{|c|c|c|c|c|c|c|}
\hline & $\begin{array}{l}\text { GROUP } \\
\text { NORMAL }\end{array}$ & $\begin{array}{l}1 \\
\text { LV }\end{array}$ & $\begin{array}{l}\text { GROUP } \\
\text { LV DYS }\end{array}$ & 2 & $\begin{array}{l}\text { GROUP } \\
\text { LV }\end{array}$ & $\begin{array}{l}3 \\
\text { ANEURYSM( }\end{array}$ \\
\hline & $\begin{array}{l}\text { diastolic } \\
\text { dysfunction } \\
(n=21)\end{array}$ & $\begin{array}{c}\text { no } \\
\text { diastolic } \\
\text { dysfunction } \\
(n=12)\end{array}$ & $\begin{array}{l}\text { diastolic } \\
\text { dysfunction } \\
(n=16)\end{array}$ & $\begin{array}{c}\text { no } \\
\text { diastolic } \\
\text { dysfunction } \\
(n=13)\end{array}$ & $\begin{array}{c}\text { diastolic } \\
\text { dysfunction } \\
(n=13)\end{array}$ & $\begin{array}{c}\text { no } \\
\text { diastolic } \\
\text { dysfunction } \\
(n=11)\end{array}$ \\
\hline $\begin{array}{l}\text { Lipoprotein } \\
\text { (a) } \\
\text { median } \\
\text { (IQR) }\end{array}$ & $\begin{array}{c}26 \\
(10-65)\end{array}$ & $\begin{array}{c}36 \\
(13-61)\end{array}$ & $\begin{array}{c}27 \\
(13-41)\end{array}$ & $\begin{array}{c}12 \\
(7-60)\end{array}$ & $\begin{array}{c}12 \\
(6-25)\end{array}$ & $\begin{array}{c}46^{\star} \\
(35-62)\end{array}$ \\
\hline
\end{tabular}




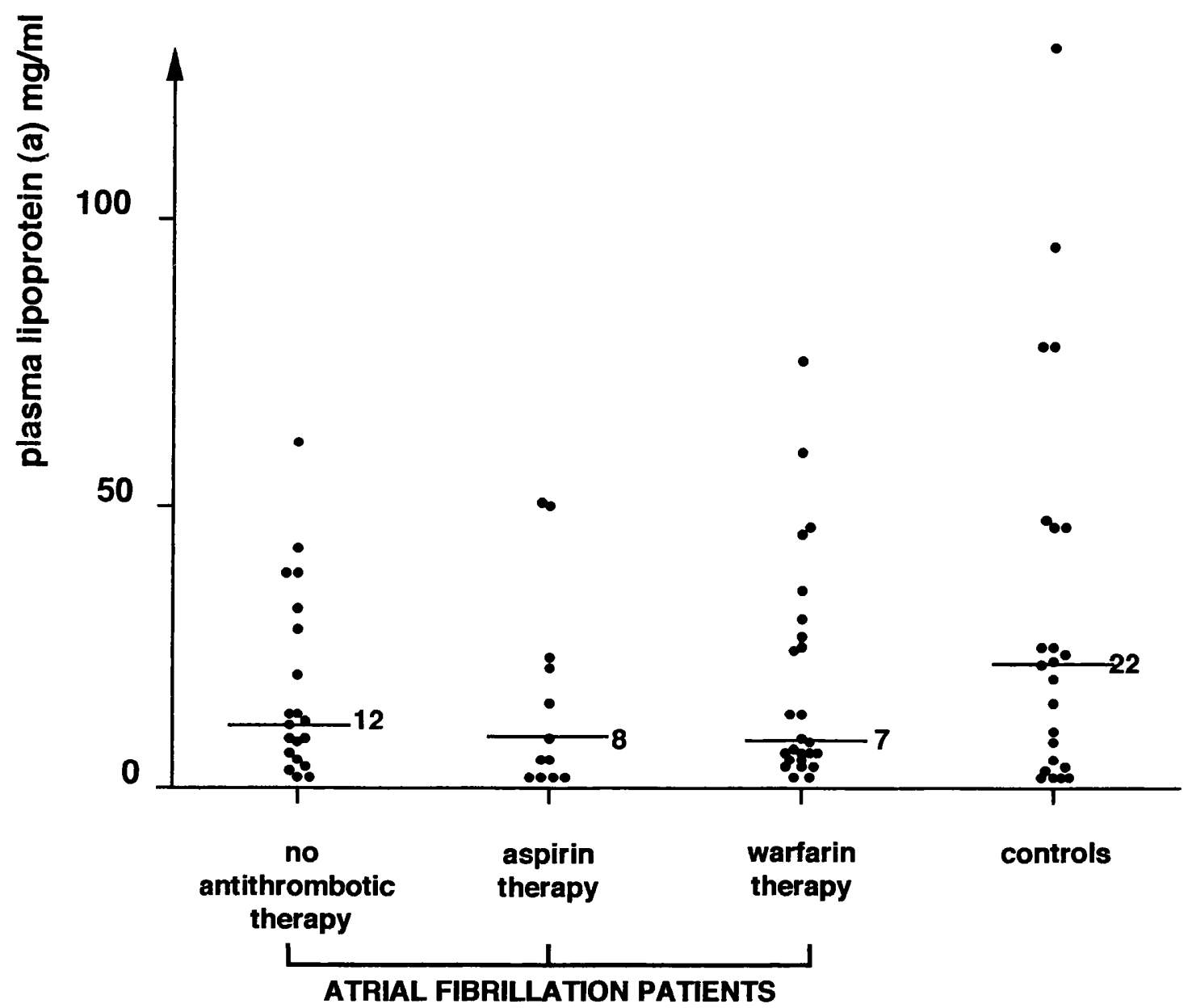

FIGURE 11.1 SERUM LIPOPROTEIN (a) AND ATRIAL FIBRILLATION 


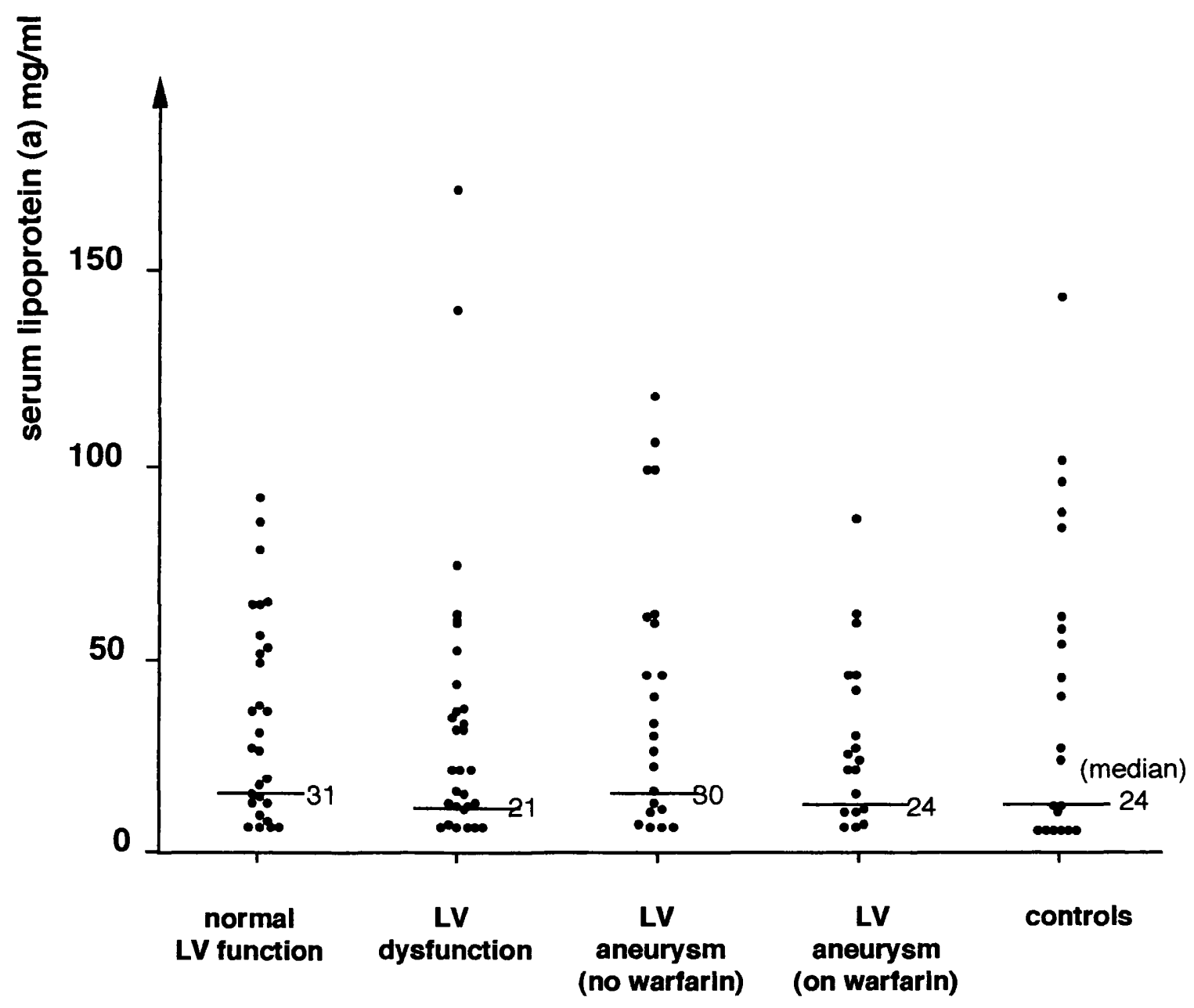

FIGURE 11.2 SERUM LIPOPROTEIN (a) AND LEFT VENTRICULAR FUNCTION 


\section{CHAPTER 12}

\section{CONCLUDING REMARKS}

AND FUTURE DIRECTIONS 
Introduction

Identification of patients at risk of cardiogenic thromboembolism poses a daunting challenge. Because of the multifactorial contributory aetiology to thromboembolic risk, it is becoming increasingly difficult to provide a cost-effective assessment of risk which is easily applicable to the clinical situation. Identification of measurable plasma or serum factors may help therefore to identify those patients at particular risk of thrombogenesis, and whom may benefit from prophylactic anticoagulant therapy. This is clinically relevant as anticoagulant therapy is not without its risks or inconvenience. I have therefore investigated the roles of fibrinogen, fibrin turnover, endothelial dysfunction and lipoprotein(a) in contributing to thrombogenesis associated with two cardiovascular conditions at high thromboembolic risk, that is, atrial fibrillation and left ventricular dysfunction.

\section{Findings in this thesis}

Atrial fibrillation was found to be a common cardiac arrhythmia amongst emergency admissions to a district general hospital. Heart failure and cerebrovascular events were the commonest presenting features, accounting for about half of all emergency admissions over a six month period who were found to be in atrial fibrillation (chapter 2). This survey gave a comprehensive profile of admissions with atrial fibrillation and how they were investigated and managed in a district general hospital. In this survey, 1 identified a suboptimal application of standard investigations in these patients. For example, two-dimensional echocardiography was infrequently used although it is established as being a useful technique for evaluating and defining structural cardiac lesions associated with atrial fibrillation and thromboembolism. There was also a reluctance to perform cardioversion or to commence anticoagulant therapy, despite the increasingly important roles for these two therapeutic interventions in the current management of atrial fibrillation. One possible reason for the low rate of introducing anticoagulation may be the uncertainty of many physicians in identifying patients at high thromboembolic risk or concerns about the adverse reactions of such therapy. The availablity of a suitable plasma marker of thrombogenesis may therefore assist decision-making in these patients. 
Factors such as fibrinogen, von Willebrand factor and lipoprotein (a) have been associated with thrombosis, embolism and stroke. In addition, the fibrin D-dimer fragment is a marker of fibrin turnover and is thus an indirect measure of intravascular thrombus formation. Present clinical data suggests, for example, that plasma fibrinogen plays a major role in the thrombogenic and atherosclerotic process; to the extent that measurement of plasma fibrinogen should probably be included in the cardiovascular risk profile. My thesis provides further evidence for this viewpoint, as I have identified abnormalities of these plasma markers suggesting a prothrombotic state in patients with atrial fibrillation and left ventricular aneurysms. A better understanding of the mechanisms involved in thrombogenesis could possibly lead to more effective methods of prevention and treatment of cardiovascular disorders.

In chapter 3, patients with chronic atrial fibrillation were found to have significant elevations of plasma fibrinogen and von Willebrand factor when compared to normal population values, and this was irrespective of aspirin or warfarin therapy. Patients with atrial fibrillation whom are on no antithrombotic therapy had the highest levels of plasma fibrin D-dimer, suggesting that these patients had the highest levels of intravascular fibrin turnover. In contrast, those whom were established on warfarin prophylaxis had the lowest fibrin Ddimer levels, consistent with the beneficial effect of warfarin in reducing thrombogenesis. No significant effect on these factors with respect to atrial size, ventricular function (as measured by echocardiography), underlying valve disease or ischaemic heart disease was noted.

Cardioversion of atrial fibrillation to sinus rhythm replaces the irregular atrial activity of atrial fibrillation with the regular atrial systole of sinus rhythm. If thrombogenesis has a simple mechanical basis, cardioversion of atrial fibrillation should be expected to immediately normalise plasma fibrinogen and fibrin D-dimer levels. This does not appear to be the case, as in patients without warfarin therapy, plasma fibrin D-dimer falls sequentially over two weeks following cardioversion (chapter 4). This is most probably partly due to the clearance of Ddimer from the circulation and that atrial mechanical function may not resume immediately. In addition, there was little change in plasma fibrinogen levels, which may in part be due to the individual variation in the return of atrial systole and small numbers in my study. Although 
clinical studies suggest a 'high risk' period for thromboembolism following cardioversion, no significant peak in plasma fibrinogen or fibrin D-dimer was noted. This is in keeping with the hypothesis that any emboli are due to pre-formed thrombus rather than formation of new thrombus following cardioversion. Patients who were anticoagulated with warfarin pre- and post-cardioversion had low plasma D-dimer levels which did not alter over the two week followup period. This is consistent with the prophylactic effects of warfarin in reducing thromboembolic risks during cardioversion.

If plasma fibrin D-dimer is a suitable indicator of intravascular thrombogenesis, further evidence may be obtained by a study of patients with paroxysmal atrial fibrillation. These patients are regarded as at intermediate thromboembolic risk when compared with chronic atrial fibrillation and sinus rhythm. In chapter 5, I demonstrated that patients with paroxysmal atrial fibrillation (on no warfarin therapy) had intermediate levels of plasma fibrinogen and fibrin D-dimer, in keeping with previous clinical observations of an intermediate thromboembolic risk. This also suggests that flow abnormalities may be important in thrombogenesis, as sustained irregular atrial activity (chronic atrial fibrillation) causes greater abnormalities in markers of thrombogenesis when compared with intermittent irregular atrial contractions (paroxysmal atrial fibrillation) and those with normal, regular atrial systolic function (sinus rhythm).

Flow abnormalities secondary to the irregular atrial activity in atrial fibrillation may account therefore for the abnormalities in the plasma levels of fibrinogen and fibrin Ddimer. To further clarify this matter, I compared patients with paroxysmal supraventricular tachycardia (whom have a paroxysmal tachycardia with normal regular atrial contractions) to matched patients manifesting paroxysmal atrial fibrillation (who have a paroxysmal tachycardia with intermittent irregular atrial activity) in chapter 6 . In this small study, patients with paroxysmal supraventricular tachycardia had lower levels (which were similar to population values) of plasma fibrinogen and fibrin D-dimer compared with matched patients having paroxysmal atrial fibrillation. This appears to confirm the importance of flow abnormalities in association with atrial fibrillation as being a major determinate of plasma fibrinogen and fibrin Ddimer levels. 
Epidemiological evidence suggests that valvular heart abnormalities significantly adds to the thromboembolic risk of patients with atrial fibrillation. However, in patients with chronic atrial fibrillation (chapter 3), I was unable to demonstrate any significant effect of valvular heart disease upon plasma fibrinogen, D-dimer or von Willebrand factor. However, all patients with valvular heart disease may not have similar risks of thromboembolism and this may be in part related to their inherent flow characteristics. In chapter 7,1 studied a small series of patients with aortic stenosis and mitral regurgitation. Both groups had higher plasma fibrinogen levels when compared with population levels, which is in keeping with flow abnormalities in these patients. However, patients with mitral regurgitation had lower plasma D-dimer levels, indicating lower intravascular fibrin turnover in these patients. This is consistent with observations that the presence of mitral regurgitation reduces spontaneous echo contrast (associated with thrombus and embolism) and intraventricular thrombus formation (for example, in patients with dilated cardiomyopathy). This also suggests that plasma fibrin D-dimer may be associated with stasis within the atrial cavity. Such stasis would contribute to thrombus formation, which appears reduced by the presence of mitral regurgitation (perhaps by a 'stirring' effect on flow). Patients with aortic stenosis, however, had similar plasma D-dimer levels to population values, in keeping with a different pathophysiology (calcific emboli and platelets) of associated emboli.

Epidemiological evidence also suggests that a further factor contributing to the risk of stroke in atrial fibrillation is the presence of heart failure. In chapter 8, I have demonstrated that patients with ischaemic heart disease had higher plasma fibrinogen and von Willebrand factor levels when compared with population controls (in keeping with present epidemiological evidence). Those with left ventricular aneurysms (as defined by radionuclide ventriculography), however, had the highest levels of plasma fibrinogen, fibrin D-dimer and von Willebrand factor. This indicates the highest risk of intravascular thrombogenesis in patients with left ventricular aneurysms and may inpart explain the association of heart failure (associated with left ventricular impairment) and thromboembolic events. As in patients with chronic atrial fibrillation, patients with left ventricular aneurysms whom were established on warfarin therapy had low fibrin D-dimer levels, consistent with a reduction in thrombogenesis. 
Clinical or epidemiological studies investigating the contribution of heart failure to thromboembolic risk do not, however, make a distinction between systolic and diastolic dysfunction. This is pertinent as $30 \%$ or more of admissions with clinical heart failure have normal systolic function. In chapter 9 , I studied the prevalence of diastolic dysfunction using echocardiography in patients with ischaemic heart disease, with and without systolic dysfunction and aneurysm formation. There was, however, no significant difference in the prevalence of diastolic dysfunction in these patient groups. In addition, there were no differences in plasma fibrinogen, fibrin D-dimer and von Willebrand factor between patients with and without diastolic dysfunction. This suggests that the flow abnormalities associated with systolic (rather than diastolic) impairment have a greater influence upon fibrinogen and fibrin turnover (and consequent thrombogenesis).

Recent prospective studies of patients with nonvalvular atrial fibrillation have shown that prophylactic anticoagulant therapy is beneficial in reducing thromboembolic risk. In chapter 10, this aspect has been investigated in patients with chronic atrial fibrillation and left ventricular aneurysms in whom anticoagulant therapy was introduced. In these patients there was a significant sequential reduction in plasma fibrin D-dimer (but not fibrinogen) levels with the introduction of warfarin therapy. In addition, in those with the highest initial D-dimer levels $(>100 \mathrm{ng} / \mathrm{ml})$ this reduction was highly significant. This is once again consistent with the beneficial effect of warfarin in reducing stroke and thromboembolism. Patients at highest risk of thrombogenesis may therefore be selected by measurement of plasma fibrin D-dimer levels.

Lipoprotein (a) is another factor associated with thrombogenesis, especially in relation to ischaemic heart disease and stroke. In chapter 11, I have attempted to determine whether there is an association between lipoprotein (a) and plasma fibrinogen and fibrin Ddimer. I therefore measured lipoprotein (a) levels in the two conditions in which I previously had demonstrated the highest intravascular fibrin turnover, that is, chronic atrial fibrillation and left ventricular aneurysms. There were, however, no significant differences between patients and healthy controls, even with warfarin therapy. This is consistent with observations that 
lipoprotein (a) is predominantly genetically determined and varies little with environmental influences.

\section{Suggestions for further study}

This thesis has identified plasma fibrin D-dimer as a potential marker of thrombus formation. Other prothrombotic markers such as fibrinogen and von Willebrand factor may also be useful in identifying those at risk of thrombogenesis and cardiovascular events. This may in turn allow the investigation of thrombogenic mechanisms in other conditions with a high thromboembolic event rate and the effects of therapeutic interventions. However, the precise clinical role of lipoprotein (a) measurement in assessing thrombogenesis remains to be demonstrated. A study of these plasma factors associated with thrombogenesis will allow further studies in risk factor modification.

For example, low-dose anticoagulant therapy has been established to reduce stroke in atrial fibrillation. There is also evidence, however, that the risk of thrombosis associated with a long-term central venous catheter was reduced by $65 \%$ in patients receiving only $1 \mathrm{mg} /$ day of warfarin, despite no measurable change in coagulation values (Bern et al 1990). This 'ultra-low-dose' warfarin may be a future area of research for stroke prevention in atrial fibrillation, the measurement of plasma D-dimer being possibly used to ensure suitable 'titration' of therapy.

Prospective studies are also required to assess the predictive nature of plasma prothrombotic markers for thromboembolic and cardiovascular risk, for example, whether or not a high D-dimer level predicts stroke or death in patients with atrial fibrillation. This aspect has recently been investigated, for example, in patients with peripheral vascular disease, where a high initial plasma D-dimer was predictive of cardiovascular events (Fowkes et al 1993). Based on current clinical evidence, most patients with chronic atrial fibrillation should now be considered for oral anticoagulation therapy, and it may no longer be reasonable or feasible (or even ethical) to investigate a prospective study of such patients without anticoagulation therapy. 
Measurement of prothrombotic factors may also give us a further understanding of the mechanisms of thrombogenesis, for example, in patients who demonstrate spontaneous echo contrast on transoesophageal echocardiography. This 'visual' evidence of stasis may be associated with the highest levels of prothrombotic markers (particularly D-dimer). This would be in keeping with experimental work associating spontaneous echo contrast with fibrinogen, platelets and rouleau formation of erythrocytes (Sigel et al 1981, Merino et al 1992).

\section{Conclusion}

Plasma levels of fibrinogen, D-dimer and von Willebrand factor are elevated in patients with chronic atrial fibrillation (without antithrombotic therapy) and patients with left ventricular aneurysms. Intermediate levels are seen in patients with paroxysmal atrial fibrillation. My thesis therefore suggests possible roles for fibrinogen, increased fibrin turnover (as measured by plasma D-dimer) and endothelial dysfunction (as reflected by von Willebrand factor) in thrombogenesis in these patients. Lipoprotein (a) appears to be little changed in these patients, with no relationship to other plasma factors, in keeping with a major genetic influence on this prothrombotic factor. In addition, levels of these markers in patients with atrial fibrillation were not significantly influenced by cardiac chamber size or structural abnormalities. In patients with left ventricular dysfunction, the presence of systolic dysfunction (but not diastolic dysfunction) and particularly left ventricular aneurysms were found to be important influences upon these prothrombotic markers.

Therapeutic intervention may also alter the tendency towards thrombogenesis. For example, cardioversion and anticoagulation both reduced plasma Ddimer levels, suggestive of the beneficial effect of these interventions. My initial survey of admissions with atrial fibrillation showed, however, that both of these procedures are infrequently considered. Physicians might therefore be aided in considering these interventions more frequently with the availability of a suitable plasma marker which could help select patients at high risk (whom would benefit most from intervention). My thesis suggests that measurement of plasma fibrin D-dimer may fulfil this role as a marker of thrombogenesis. 
Measurement of other factors such as fibrinogen and von Willebrand factor will also contribute to the overall cardiovascular risk profile, allowing the risk stratification for thrombogenesis in such patients. 


\title{
APPENDIX 1 \\ PUBLICATIONS AND PRESENTATIONS ARISING FROM
}

\author{
WORK OF THIS THESIS
}

\author{
Publications: \\ Lip GYH, Tean KN, Dunn FG. "Atrial fibrillation in a district general hospital" \\ - British Heart Journal ; in press. \\ Lip GYH, Metcalfe MJ, Rae AP. "Management of paroxysmal atrial fibrillation." \\ -Quarterly Journal of Medicine 1993; 86: 467-72.
}

\section{Presentations:} May 1992

August 1992

November 1992

December 1992

March 1993

April 1993
-"Increased plasma fibrinogen and D-dimer levels and left atrial size in patients with chronic atrial fibrillation."

Lip,G.Y.H.,Rumley, A.,Lowe, G.D.O.,Dunn, F.G.

Oral presentation to the Scottish Society for Experimental Medicine

meeting on 15th May, 1992 at Aberdeen Royal Infirmary.

Scottish Medical Journal 1993; 37(6):189 (abstract).

-"Increased plasma fibrinogen and D-dimer levels and left atrial size in patients with chronic atrial fibrillation."

Lip,G.Y.H.,Rumley, A.,Lowe, G.D.O.,Dunn, F.G.

Presentation to the XIVth Congress of the European Society of Cardiology meeting at Barcelona, Spain; August 30 - September 31992.

European Heart Journal 1992;13 (Abstract Supplement): 66.

-"Effect of warfarin and aspirin therapy on plasma fibrinogen and fibrin D-dimer levels in patients with chronic atrial fibrillation."

Lip,G.Y.H., Rumley, A., Metcalfe, M. J., Dunn, F.G. , Lowe, G.D.O.

Presentation to the Winter Meeting of the Medical Research Society

at the Royal Colege of Physicians, London; November 5-6,1992.

Clinical Science 1993; Suppl 27: 17P (Poster No.62)

-"Increased plasma fibrinogen in patients with ischaemic heart disease and left ventricular dysfunction."

Lip,G.Y.H.,Metcalfe, M.J., Rumley, A., Hogg, K. J., Rae, A.P., Lowe, G.D.O.,Dunn, F.G.

Oral presentation to the Scottish Society for Experimental Medicine meeting on 4th December, 1992 at The Walton Conference Centre, Southern General Hospital, Glasgow.

Scottish Medical Journal 1993; 38: 89 (abstract).

-" Increased plasma fibrinogen and fibrin D-dimer levels in patients with chronic atrial fibrillation: The effects of Antithrombotic Therapy."

Lip,G.Y.H., Rumley, A., Dunn, F.G. , Lowe, G.D.O.

Oral presentation to the American College of Cardiology (ACC'93)

42nd Annual Scientific Session, at the Anaheim Convention Center,

Anaheim, California, U.S.A.; 14-18 March, 1993.

Journal of the American College of Cardiology 1993; 21: 479A

(abstract 831-5).

-"Increased plasma fibrinogen levels in patients with atrial fibrillation:

A rheological mechanism for thromboembolic risk ?"

Lip,G.Y.H., Metcalfe, M. J., Rumley, A., Lowe, G.D.O., Dunn, F.G.

Presentation to the Spring Meeting of the Medical Research Society at University College Medical School, London; April 21 \& 22, 1993. Clinical Science 1993; Suppl 29: 24P (Poster No.81)

-"Increased plasma fibrinogen and D-dimer levels in patients with 
left ventricular aneurysms: A mechanism for thromboembolism ?" Lip,G.Y.H., Metcalfe, M.J., Rumley, A., Lowe, G.D.O.,Dunn, F.G. Presentation to the Spring Meeting of the Medical Research Society at University College Medical School, London; April 21 \& 22, 1993. Clinical Science 1993; Suppl 29: 24P (Poster No.80)

May 1993

-"An audit of atrial fibrillation in a district general hospital." Lip,G.Y.H., Tean K.N., Dunn, F.G.

Presentation to the British Cardiac Society Annual Meeting meeting 18-21 May, 1993 at the Wembley Conference and Exhibition Centre, London HA9 ODW. (Poster No. 679)

British Heart Journal 1993; 69 (Suppl) P52 (abstract 228).

May1993

-"Plasma fibrinogen and fibrin D-dimer levels in patients with coronary artery disease and left ventricular aneurysms."

Lip,G.Y.H. ,Metcalfe, M.J., Rumley, A., Hogg, K. J., Rae, A.P., Dunn, F.G. , Lowe, G.D.O.

Presentation to the British Cardiac Society Annual Meeting meeting 18-21 May, 1993 at the Wembley Conference and Exhibition Centre, London HA9 ODW. (Poster No. 138)

British Heart Journal 1993; 69 (Suppl): P39 (abstract 177).

May1993

-"Increased plasma fibrinogen levels in patients with chronic and paroxysmal atrial fibrillation: A mechanism for thromboembolic risk ?" Lip,G.Y.H. , Metcalfe, M.J., Rumley, A., Lowe, G.D.O., Dunn, F.G. Oral presentation to the British Cardiac Society Annual Meeting meeting 18-21 May, 1993 at the Wembley Conference and Exhibition Centre, London HA9 ODW. (Abstract No. 110)

British Heart Journal 1993; 69 (Suppl): P22 (abstract 65).

May 1993

-"Is the hospital management of atrial fibrillation optimal?"

Lip,G.Y.H., Tean K.N., Dunn, F.G.

Presentation to the Scottish Society for Experimental Medicine meeting on 28th May 1993 at the Western General Hospital, Edinburgh. Scottish Medical Journal 1993; in press (abstract).

May $1993 \quad$-Elevated plasma fibrinogen levels in patients with chronic and paroxysmal atrial fibrillation: Rheological evidence for an increased thromboembolic risk?"

Lip,G.Y.H., Rumley, A., Metcalfe, M.J., Dunn, F.G., Lowe, G.D.O. Presentation to the Scottish Society for Experimental Medicine meeting on 28th May 1993 at the Western General Hospital, Edinburgh. Scottish Medical Journal 1993; in press (abstract).

November 1993 -"Cardioversion of atrial fibrillation reduces intravascular fibrin turnover and thrombogenesis."

Lip,G.Y.H., Metcalfe, M.J., Rumley, A., Dunn, F.G., Lowe, G.D.O.

Presentation to the Winter Meeting of the Medical Research Society at the Royal Colege of Physicians, London; November 11-12,1993. Clinical Science 1994; Suppl 30 :34P (Poster No: 110)

November 1993 -"Does endothelial dysfunction contribute to thrombogenesis in patients with chronic atrial fibrillation?"

Lip,G.Y.H., Smith, D., Lowe, G.D.O., Dunn, F.G.

Presentation to the Winter Meeting of the Medical Research Society at the Royal Colege of Physicians, London; November 11-12,1993. Clinical Science 1994; Suppl 30 :34P (Poster No: 111)

November 1993 -"A survey of atrial fibrillation amongst acute medical admissions to a district general hospital."

Lip,G.Y.H.,Tean, K.N.,Dunn, F.G.

Presentation to the Winter Meeting of the Medical Research Society at the Royal Colege of Physicians, London; November 11-12,1993. 
Clinical Science 1994; Suppl 30 : 34P-35P(Poster No: 112)

December 1993 -"Is lipoprotein (a) increased in patients at high risk of thrombogenesis?" Lip,G.Y.H., Metcalfe, M.J., Rolton, H., Farish, E., Lowe G.D.O., Dunn, F.G. Presentation to the Scottish Society for Experimental Medicine meeting on 3rd December 1993 at the Wolfson Lecture Theatre, Ninewells Hospital and Medical School, Dundee.

Scottish Medical Journal 1994; in press (abstract).

December 1993 Lip,G.Y.H., Rumley, A., Dunn, F.G., Lowe G.D.O. "Fibrin D-dimer levels in patients with atrial fibrillation and left ventricular aneurysms: effects of introducing anticoagulant therapy on thrombogenesis." Presentation to the Scottish Society for Experimental Medicine meeting on 3rd December 1993 at the Wolfson Lecture Theatre, Ninewells Hospital and Medical School, Dundee.

Scottish Medical Journal 1994; in press (abstract). 


\section{APPENDIX 2}

\section{REFERENCES}

Abe A, Maeda S, Makino K, Okuno M, Shimokawa K, Noma A, Kawade M. [Sequential changes in plasma lipoprotein (a) as acute phase protein after myocardial infarction and surgery group]. Rinsho Byori 1989; 37: 1038-44.

Al-Zahrani H, Lowe GDO, Douglas JT, Cuschieri R, Pollock JG, Smith WCS. Increased fibrin turnover in peripheral arterial disease: comparison with a population study. Clin Hemorheol 1992; 12: 867-72.

Alam $M$, Thorstrand $C$. Left ventricular function in patients with atrial fibrillation before and after cardioversion. Am J Cardiol 1992; 69: 694-96.

Albers J, Taggart H, Applebaum-Bowden D et al. Reduction of lecithin-cholesterol transferase, apolipoprotein $\mathrm{D}$, and the $\mathrm{Lp}(\mathrm{a})$ lipoprotein with the anabolic steroid stanzolol. Biochim Biophys Acta 1984; 795: 293-6.

Alpert JS, Petersen P, Godttredsen J. Atrial fibrillation: natural history, complications, and management. Ann Rev Med 1988;39:41-52.

Alpert MA. Mitral valve prolapse. Br Med J 1993; 306: 943-44.

Anderson JL, Gilbert EM, Alpert BL et al. Prevention of symptomatic recurrences of paroxysmal atrial fibrillation in patients initially tolerating antiarrhythmic therapy: a multicenter, double-blind, crossover study of flecainide and placebo with transtelephonic monitoring. Circulation 1989; 80: 1557-70.

Antiplatelet Trialists' Collaboration. Secondary prevention of vascular disease by prolonged antiplatelet treatment. Br Med J 1988; 296: 320-31.

Appleton CP, Hatle LK, Popp RL. Demonstration of restrictive ventricular physiology by Doppler echocardiography. J Am Coll Cardiol 1988; 11: 757-68.

Appleton CP, Hatle LK, Popp RL. Relation of transmitral flow velocity patterns to left ventricular diastolic function: new insights from a combined hemodynamic and Doppler echocardiographic study. J Am Coll Cardiol 1988; 12: 426-40.

Armstrong VW, Neubauer C, Schutz E, Tebbe U. Lack of association between raised serum $\mathrm{Lp}$ (a) concentration and unsucessful thrombolysis after myocardial infarction. Lancet 1990; 336: 1077.

Armstrong VW, Schleef $J$, Thiery $J$ et al. Effect of HELP-LDL-apheresis on serum concentrations of human lipoprotein (a): kinetic analysis of the post-treatment return to baseline levels. Eur J Clin Invest 1989; 19: 235-40.

Arnold AZ, Mick MJ, Mazurek RP, Loop FD, Trohman RG. Role of prophylactic anticoagulation for direct current cardioversion in patients with atrial fibrillation or atrial flutter. J Am Coll Cardiol 1992; 19: 851-5.

Atwood JE, Myers J, Sullivan M, Forbes S, Sandhu S, Callaham P, Froelicher V. The effect of cardioversion on maximal exercise capacity in patients with chronic atrial fibrillation. Am Heart $J$ 1989; 118: 913-18.

Badimon L, Badimon JJ, Fuster V. Pathogenesis of thrombosis. In: Fuster V, Verstraete M (ed) Thrombosis in cardiovascular disorders. Philadelphia. WB Saunders. 1992;17-39.

Balleisen L, Bailey J, Epping PH, Schulte H, van de Loo J. Epidemiological study on Factor VII, Factor VIII and Fibrinogen in an industrial population: I. Baseline data on the relation to 
age, gender, body-weight, smoking, alcohol, pill-using, and Menopause. Thromb Haemost 1985; 54: 475-79.

Baller D, Miche E, Hurtado R, Weibe G, Prohaska W, Gleichmann. Relation of fibrinogen to the extent of coronary artery disease. Thromb Haemost 1991; 65: 993.

Banerjee AK, Pearson J, Gilliland EL, Goss D, Lewis JD, Stirling Y, Meade TW. A six year prospective study of fibrinogen and other risk factors associated with mortality in stable claudicants. Thromb Haemostat 1992; 68: 261-63.

Bath PMW, Prasad A, Brown MM, MacGregor GA. Survey of use of anticoagulation in atrial fibrillation. Br Med J 1993; 307: 1045.

Bathen J, Sparr S, Rokseth R. Embolism in sinoatrial disorder. Acta Med Scand 1978; 203: 711.

Beppu S, Nimura Y, Sakakibara H, Nagata S, Park Y-D, Izumi S. Smoke-like echo in the left atrial cavity in mitral valve disease: its features and significance. J Am Coll Cardiol 1985; 6 : 744-9.

Beppu S, Park YD, Sakakibara H, Nagata S, Numura Y. Clinical features of intracardiac thrombosis based on echocardiographic observation. Jpn Circ J 1984; 48: 75-82.

Bern MM, Lokich JJ, Wallach SR, Bothe A, Benotti PN, Arkin CF, Greco FA, Huberman M, Moore C. Very low doses of warfarin can prevent thrombosis in central venous catheters. Ann Intern Med 1990; 112: 423-28.

Bimmermann A, Boerschmann C, Schwartzkopff W, Von Baeyer H, Schleicher J. Effective therapeutic measures for reducing lipoprotein (a) in patients with dyslipidemia. Lipoprotein (a) reduction with sustained-release bezafibrate. Curr Therapeu Res 1991; 49: 635-43.

Bini A, Kudryk BJ. Fibrinogen, Fibrin and Atherosclerosis: Vascular and systemic effects. In: Ernst E, Koenig W, Lowe GDO, Meade T (eds). Fibrinogen : A 'new' cardiovascular risk factor. Wien: Blackwell-MZV. 1992; 41-45.

Bjerkelund CJ, Orning OM.The efficacy of anticoagulant therapy in preventing embolism related to D.C. electrical cardioversion of atrial fibrillation. Am J Cardiol 1969;23:208-216.

Black IW, Hopkins AP, Lee LC, Walsh WF, Jacobson BM. Left atrial Spontaneous Echo Contrast: A Clinical and Echocardiographic Analysis. J Am Coll Cardiol 1991; 18: 398-404.

Bland JM, Altman DG. Statistical methods for assessing agreement between two methods of clinical measurement. Lancet 1986; 1:307-10.

Blann AD, Naqvi T, Waite M, McCollum CN. Von Willebrand factor and endothelial damage in essential hypertension. J Hum Hypertens 1993; 7: 107-111.

Blevins RD, Kerin NZ, Benaderet D, Frumin H, Faitel K, Jarandilla R, Rubenfire M. Amiodarone in the management of refractory atrial fibrillation. Arch Intern Med 1987; 147: 1401-4.

Boerwinkle E, Menzel HJ, Kraft HG, Utermann G. Genetics of the quantitative Lp(a) lipoprotein trait. III. Contribution of $L p(a)$ glycoprotein phenotypes to normal lipid variation. Hum Genet 1989; 82: 73-8.

Bogousslavsky J, Regli F. Ischemic stroke in adults younger than 30 years of age. Cause and prognosis. Arch Neurol 1987; 44: 479-82.

Bonaduce D, Breglio R, Conforti G, De Luca N, Montemurro MV, Arrichiello P, Petretta M. Myocardial hypertrophy and left ventricular diastolic function in hypertensive patients: an echo Doppler evaluation. Eur Heart J 1989; 10: 611-21.

Boneu B, Abbai M, Plante J, Bierme R.Factor VIII complex and endothelial damage.Lancet 1975; I:1430. 
Bonithon-Kopp C, Scarabin PY, Darne B, Malmejac A, Guize L. Menopause-related changes in lipoproteins and some other cardiovascular risk factors. Int J Epidemiol 1990; 19: 42-8.

Bonow RO, Bacharach SL, Green MV, Kent KM, Rosing DR, Lipson LC, Leon MB, Epstein SE. Impaired left ventricular diastolic filling in patients with coronary artery disease: assessment with radionuclide angiography. Circulation 1981; 64: 315-23

Bonow RO. Regional left ventricular nonuniformity. Effects on left ventricular diastolic function in ischemic heart disease, hypertrophic cardiomyopathy, and the normal heart. Circulation 1990; 81 (Suppl III): III-54-III-65.

Boston Area Anticoagulation Trial for Atrial Fibrillation Investigators. The effect of low-dose warfarin on the risk of stroke in patients with non-rheumatic atrial fibrillation. $\mathrm{N}$ Engl $\mathrm{J}$ Med 1990; 323: 1505-11.

Brand FN, Abbott RD, Kannel WB, Wolf PF. Characteristics and prognosis of lone atrial fibrillation: 30-year follow up in the Framingham study. JAMA 1985; 254: 3449-53.

Braunwald E. Valvular heart disease. In: Braunwald E (ed) Heart Disease. A textbook of cardiovascular medicine. Philadelphia: 4th ed. WB Saunders.1993; 1007-77.

Brenner B, Zwang E, Bronshtein M, Seligsohn U. Von Willebrand factor multimer patterns in pregnancy-induced hypertension. Thromb Haemost 1989; 62: 715-7.

Broadhurst P, Kelleher C, Hughes L, Imeson JD, Raftery EB. Fibrinogen, factor VII clotting activity and coronary artery disease severity. Atherosclerosis 1990; 85: 169 - 73

Broch OJ, Müller O. Haemodynamic studies during auricular fibrillation and after restoration of sinus rhythm. Br Heart J 1957; 19: 222-26.

Brodsky MA, Allen BJ, Capparelli EV, Luckett CR, Morton R, Henry WL. Factors determining maintainence of sinus rhythm after chronic atrial fibrillation with left atrial dilatation. Am J Cardiol 1989; 63: 1065-68.

Brogan WC III, Hillis LD, Flores ED, Lange RA. The natural history of isolated left ventricular diastolic dysfunction. Am J Med 1992; 92: 627-30.

Cabin HS, Clubb S, Hall C, Perlmutter RA, Feinstein AR. Risk for systemic embolization of atrial fibrillation without mitral stenosis. Am J Cardiol 1990; 65: 1112-16.

Cabin HS, Roberts WC. Left ventricular aneurysm, intraaneurysmal thrombus and systemic embolus in coronary artery disease. Chest 1980; 77: 586-90.

Cairns JA, Connolly SJ. Nonrheumatic atrial fibrillation. Risk of stroke and role of antithrombotic therapy. Circulation 1991; 84: 469-81.

Caplan LR, D'Cruz I, Hier DB, Reddy H, Shah S. Atrial size, atrial fibrillation and stroke. Ann Neurol 1986; 19: 158-61.

Capucci A, Boriani G [ldiopathic atrial fibrillation:Clinical and therapeutic problems.] Cardiologia 1991; 36 (8 Suppl); 17-23.

Carlson LA, Hamsten A, Asplund A. Pronounced lowering of serum levels of lipoprotein (a) in hyperlipidaemic subjects treated with nicotinic acid. J Intern Med 1989; 226: 271-6.

Castello R, Pearson AC, Labovitz AJ. Prevalence and clinical implications of atrial spontaneous echo contrast in patients undergoing transesophageal echocardiography. Am J Cardiol 1990; 65: 1149-53.

Cerebral Embolism Task Force. Cardiogenic brain embolism: The second report of the Cerebral Embolism Task Force. Arch Neurol 1989; 46: 727-43. 
Chang HJ, Bell JR, Deroo DB, Kirk JW, Wasson JH Physician variation in anticoagulating patients with atrial fibrillation. Arch Intern Med 1990; 150: 83-86.

Chen YT, Kan MN, Chen JS, Lin WW, Hwang DS, Chang M, Lee DY, Hwang SL, Chiang BN. Contributing factors to formation of left atrial spontaneous echo contrast in mitral valvular disease. J Ultrasound Med 1990; 9: 151-55.

Cheng TO. Cardiac failure in coronary heart disease. Am Heart J 1990; 120: 396-412.

Chesebro JH, Fuster V, Halperin JL Atrial fibrillation - risk marker for stroke. N Engl J Med 1990; 323: 1556-58.

Chevalier $\mathrm{H}$. Spontaneous resumption of sinus rhythm in an elderly patient after 13 years of permanent atrial fibrillation. Am Heart J 1979; 98: 361-5.

Clark A, Cotter L. Cardioversion in atrial fibrillation. Br J Hosp Med 1993; 49: 256-61.

Clarkson TB, Kaplan JR, Adams MR, Manuck SB. Psychosocial influences on the pathogenesis of atherosclerosis among nonhuman primates. Circulation 1987; 76 (suppl I): I$29-1-40$.

Cleland JGF, Sbarouni E, Oakley CM, Sutton G, Fletcher A, Bulpitt C, Poole-Wilson P. Prophylaxis against thrombo-embolic events: Results of a survey of British Cardiologists. $\mathrm{Br}$ Heart J 1993; 69 (Suppl): 51 (abstr).

Cohen BA, Hildebrand F, Biller J. Cardioembolic Stroke: Part 1 - Clinical patterns and asssociated cardiac conditions. ACC Current Journal Review 1993; 2: 51-52.

Cohen JC, Chiesa G, Hobbs HH. Sequence polymorphisms in the apolipoprotein (a) gene. J Clin Invest 1993; 91: 1630-36.

Cohn JN, Johnson G. Heart failure with normal ejection fraction. The V-HeFT Study Circulation 1990; 81 (Suppl III); III-48- III- 53.

Collier A, Rumley A, Rumley AG, Paterson JR, Leach JP, Lowe GDO, Small M. Free radical activity and hemostatic factors in NIDDM patients with and without microalbuminuria. Diabetes 1992; 41: 909-13.

Connelly JB, Cooper JA, Meade TW. Strenuous exercise, plasma fibrinogen, and factor VII activity. Br Heart J 1992; 67: 351-4.

Connolly SJ, Hoffert DL. Usefulness of propafenone for recurrent paroxysmal atrial fibrillation. Am J Cardiol 1989; 63: 817-19.

Connolly SJ, Laupacis A, Gent M, Roberts RS, Cairns JA, Joyner C. Canadian Atrial Fibrillation Anticoagulation (CAFA) Study. J Am Coll Cardiol 1991; 18: 349-55.

Coplen SE, Antmann EM, Berlin JA, Hewitt P, Chalmers TC. Efficacy and safety of quinidine therapy for maintainence of sinus rhythm after cardioversion: a meta-analysis of randomized control trials. Circulation 1990; 82: 1106-16.

Corbalan R, Arriagada D, Braun S, Tapia J, Huete I, Kramer A, Chavez A. Risk factors for systemic embolism in patients with paroxysmal atrial fibrillation. Am Heart $J$ 1992; 124: 14953.

Coull BM, Beamer N, De Garmo P, Sexton G, Nordt F, Knox R, Seaman GV. Chronic blood hypeviscosity in subjects with acute stroke, transient ischaemic attack, and risk factors for stroke. Stroke 1991; 22: 162-8.

Cox JL, Boineau JP, Schuessler RB, Ferguson TB Jr, Cain ME, Lindsay BD et al. Successful surgical treatment of atrial fibrillation. Review and clinical update. JAMA 1991; 266: 1976-80. 
Craveri A, Tornaghi G, Paganardi L, Ranieri R, Leonardi G, Di Bella M. (Hemorrheologic disorders in obese patients. Study of the viscosity of the blood, erythrocytes, plasma, fibrinogen and the erythrocyte filteration index.) Minerva Med 1987; 78: 899-906.

Cregler LL. Antithrombotic therapy in left ventricular thrombosis and systemic embolism. Am Heart J 1991; 123: 1110-4.

Cressman MD, Heyka RJ, Paganini EP, O'Neil J, Skibinski Cl, Hoff HF. Lipoprotein (a) is an independent risk factor for cardiovascular disease in hemodialysis patients. Circulation 1992; 86: $475-82$.

Crijns HJ, Van Gelder IC, Van Gilst WH, Hillege H, Gosslink AM, Lie KI. Serial antiarrhythmic drug treatment to maintain sinus rhythm after electrical cardioversion for chronic atrial fibrillation or atrial flutter. Am J Cardiol 1991; 68: 335-41.

Dahlen GH, Guyton JR, Attar M, Farmer JA, Kautz JA, Gotto AM. Association of levels of lipoprotein $L p(a)$, plasma lipids, and other lipoproteins with coronary artery disease documented by angiography. Circulation 1986; 74: 758-65.

Dalzell GWN, Anderson J, Adgey AAJ. Factors determining success and energy requirements for cardioversion of atrial fibrillation. Q J Med 1990; 76: 903-13.

Daniel WG, Nellessen U, Schroder E, Nonnast-Daniel B, Bednarski P, Nikutla P, Lichtlen PR. Left atrial spontaneous echo contrast in mitral valve disease: an indicator for an increased thromboembolic risk. J Am Coll Cardiol 1988; 11: 1204-11.

Davidson E, Rotenberg Z, Weinberger I, Fuchs J, Agmon J. Diagnosis and characteristics of Ione atrial fibrillation. Chest 1989; 95: 1048-50.

de Knijff P, Kaptein A, Boomsma D, Proncen HMG, Frants RR, Havekes LM. Apolipoprotein E polymorphism affects plasma levels of lipoprotein (a). Atherosclerosis 1991; 90: 169-74.

de Nooijer C, Sparling CM. Quinidine treatment of chronic lone atrial fibrillation. Clin Cardiol 1990; 13: 711-14.

Declerck PJ, Mombaerts P, Holvoet P, De Mol M, Collen D. Fibrinolytic response and fibrin fragment D-dimer levels in patients with deep venous thrombosis. Thromb Haemost 1987; 58: 1024-9.

Defauw JJ, Guiraudon GM, van Hemel NM, Vermeulen FE, Kingma JH, de Bakker JM. Surgical therapy of paroxysmal atrial fibrillation with the "corridor" operation. Ann Thorac Surg 1992; 53: $564-70$.

Delemarre BJ, Visser CA, Bot $H$, Dunning AJ. Prediction of apical thrombus formation in acute myocardial infarction based on left ventricular spatial flow pattern. J Am Coll Cardiol 1990; 15: 355-60.

Dethy M, Chassat C, Roy D, Mercier LA. Doppler echocardiographic predictors of recurrence of atrial fibrillation after cardioversion. Am J Cardiol 1988; 62: 723-6.

Devereux RB. Left ventricular diastolic dysfunction: Early diastoic relaxation and late diastolic compliance. J Am Coll Cardiol 1989; 13: 337-39.

Dittrich HC, Erickson JS, Schneidermen T, Blacky AR, Savides T, Nicod PH. Echocardiographic and clinical predictors for outcome of elective cardioversion of atrial fibrillation. Am J Cardiol 1989; 63: 193-97.

Dormandy J, Ernst E, Matrai A, Flute PT. Hemorrheological changes following acute myocardial infarction. Am Heart J 1982; 104: 1364-7.

Dormandy JA, Hoare E, Colley J, Arrowsmith DE, Dormandy TL. Clinical, haemodynamic, rheological and biochemical findings in 126 patients with intermittent claudication. $\mathrm{Br}$ Med $\mathrm{J}$ 1973; 4: 576-81. 
Dougherty AH, Naccarelli GV, Gray EL, Hicks $\mathrm{CH}$, Goldstein RA. Congestive heart failure with normal systolic function. Am J Cardiol 1984; 54: 778-82.

Drayna DT, Hegele RA, Hass PE, Emi M, Wu LL, Eaton DL, Lawn RM, Williams RR, White RL, Lalouel JM. Genetic linkage between lipoprotein (a) phenotype and a DNA polymorphism in the plasminogen gene. Genomics 1988;3: 230-6

Duguid JB. Thrombosis as a factor in the pathogenesis of coronary atherosclerosis. J Pathol Bacteriol 1946; 58: 207-12.

Dunn M, Alexander J, de Silva R, Hildner F. Antithrombotic therapy in atrial fibrillation. Chest 1989; 95: 118S-27S

ECAT Angina Pectoris Study Group. ECAT angina pectoris study: baseline associations of haemostatic factors with extent of coronary arteriosclerosis and other coronary risk factors in 3000 patients with angina pectoris undergoing coronary angiography. Eur Heart $\mathrm{J} 1993 ; 14$ : 8-17.

Echt DS, Liebson PR, Mitchell LB et al. Mortality and morbidity in patients receiving encainide, flecainide or placebo. N Engl J Med 1991; 324: 781-8.

Edelberg JM, Gonzales-Gronow M, Pizzo SV. Lipoprotein (a) inhibits streptokinase-mediated activation of human plasminogen. Biochemistry 1989; 28: 2370-74.

Egorov DF, Vygovskii AB, Podlesov AM, lashin SM. [Destruction of the atrioventricular junction and implantation of synchronized atrial pacemaker in a patient with paroxysmal atrial fibrillation.] Klin Med (Mosk) 1990; 68: 88-9.

Elwood PC, Yarnell JWG, Pickering J, Fehily AM, O'Brien JR. Exercise, fibrinogen and other risk factors for ischaemic heart disease. Br Heart J 1993; 69: 183-87.

Ernst E, Koenig W, Lowe GDO, Meade T (eds). Fibrinogen : A 'new' cardiovascular risk factor. Wien: Blackwell-MZV, 1992.

Ernst E, Resch KL, Matrai A, Buhl M, Schlosser P, Paulsen HF. Impaired blood rheology : a risk factor for stroke? J Intern Med 1991; 229: 457-62.

Ernst E, Resch KL. Fibrinogen as a cardiovascular risk factor: a meta-analysis and review of the literature. Ann Intern Med 1993; 118: 956-63.

Ernst E, Schmolzl, Matrai A, Schramm W. Hemorheological effects of oral contraceptives. Contraception 1989; 40: 571-80.

Ernst E. Fibrinogen. Br Med J 1991; 303: 596-97.

Ernst E. Regional variations in plasma fibrinogen levels. Ann Intern Med 1991; 115: 329-30.

Etingin OR, Hajjar DP, Hajjar KA, Harpel PC, Nachman RL. Lipoprotein (a) regulates plasminogen activator inhibitor-1 expression in endothelial cells. A potential mechanism in thrombogenesis. J Biol Chem 1991; 266: 2459-65.

Ezekowitz MD, Bridgers SL, James KE et al. Warfarin in the prevention of stroke associated with nonrheumatic atrial fibrillation. N Engl J Med 1992; 327: 1406-12.

Ezekowitz MD, Wilson DA, Smith EO et al Comparasion of indium-111 platelet scintigraphy and two-dimensional echocardiography in the diagnosis of left ventricular thrombi. N Engl J Med 1982; 306: 1509-13.

Fairfax AJ, Lambert CD, Leatham A. Systemic embolism in chronic sinoatrial disorder. N Engl J Med 1976; 295: 190- 92. 
Falk RH, Knowlton AA, Bernard SA, Gotlieb NE, Battinelli NJ. Digoxin for converting recentonset atrial fibrillation to sinus rhythm. A randomised, double-blinded trial. Ann Intern Med 1987; 106: 503-5.

Falk RH, Leavitt JI. Digoxin for atrial fibrillation: a drug whose time has gone? Ann Intern Med 1991; 114: 573-5

Farrer M, Game FL, Albers CJ, Neil HAW, Winocour PH, Laker MF, Adams PC, Alberti KGMM. Association between impaired glucose tolerance and circulating concentration of $\mathrm{Lp}(\mathrm{a})$ lipoprotein in relation to coronary heart disease. Br Med J 1993; 307: 832-36.

Faxon DP, Ryan TJ, Davis KB, McCabe CH, Myers W, Lesperance J, Shaw R, Tong TGL. Prognostic significance of angiographically documented left ventricular aneurysm from the coronary artery surgery study (CASS). Am J Cardiol 1982; 50: 157-64.

Feely J, Barry M, Keeling PWN, Weir DG, Cooke T. Lipoprotein (a) in cirrhosis. Br Med J 1992; 304: 545-46.

Finkelhor RS, Sun JP, Castellanos M, Bahler RC. Predicting left heart failure after a myocardial infarction: a preliminary study of the value of echocardiographic measures of left ventricular filling and wall motion. J Am Soc Echocardiogr 1991; 4: 215-23.

FitzGerald GA, Oates JA, Nowak J. Cigarette smoking and hemostatic function. Am Heart J 1988; 115: 267-271.

Flegel KM, Hanley J. Risk factors for stroke and other embolic events in patients with nonrheumatic atrial fibrillation. Stroke 1989; 20: 1000-4

Forfar JC, Miller HC, Toft AD. Occult thyrotoxicosis: A correctable cause of 'idiopathic ' atrial fibrillation Am J Cardiol 1979; 44: 9-12.

Forfar JC, Toft AD. Thyrotoxic atrial fibrillation: an underdiagnosed condition ? Br Med J 1982: 285: 909-10.

Fowkes FGR, Connor JM, Smith FB, Wood J, Donnan PT, Lowe GDO. Fibrinogen genotype and risk of peripheral atherosclerosis. Lancet 1992; 339: 693-96.

Fowkes FGR, Lowe GDO, Housley E, Rattray A, Rumley A, Elton RA, MacGregor IR, Dawes J. Cross-linked fibrin degradation products, progression of peripheral arterial disease, and risk of coronary heart disease. Lancet 1993; 342: 84-86.

Friedman BJ, Drinkovic N, Miles H, Shin W, Mazzoleni A, DeMaria AN. Assessment of left ventricular diastolic function: comparasion of Doppler echocardiography and gated blood pool scintigraphy. J Am Coll Cardiol 1986; 8: 1348-54.

Friedman PJ. Atrial fibrillation after stroke in the elderly. Stroke 1991; 22: 209-14.

Furui H, Taniguchi N, Yamauchi K, Sotobata I, Saito H, Inagaki H. Effects of treadmill exercise on platelet function, blood coagulability and fibrinolytic activity in patients with atrial fibrillation. Jpn Heart J 1987; 28: 177-84.

Galun E, Flugelman MY, Glickson M, Eliakim M. Failure of long-term digitalization to prevent rapid ventricular response in patients with paroxysmal atrial fibrillation. Chest 1991; 99: 103840.

Ganda OP, Arkin CF. Hyperfibrinogenemia: A major determinant of vascular complications in diabetes. In: Ernst E, Koenig W, Lowe GDO, Meade T (eds). Fibrinogen : A 'new' cardiovascular risk factor. Wien: Blackwell-MZV. 1992; 254-58.

Garcia Frade LJ, Alvarez JJ, Rayp I, Torrado MC, Lasuncion MS, Avello AG, Hernandez A, Marin E. Fibrinolytic parameters and lipoprotein (a) levels in plasma of patients with coronary artery disease. Thromb Res 1991; 63: 407-18. 
Garcia- Bolao I, Alegria E, Iglesias I, Velasco S, Alzamora P, Fidalgo ML.(Contraceptives and ischemic cardiopathy.) Rev Med Univ Navarra 1990; 34: 144-50.

Geggel RL, Carvalho AC, Hoyer LW, Reid LM. von Willebrand factor abnormalities in primary pulmonary hypertension. Am Rev Respir Dis 1987; 135: 294-9.

Genest J Jr, Jenner JL, McNamara JR, Ordovas JM, Silberman SR, Wilson PWF, Schaefer EJ. Prevalence of lipoprotein (a) [Lp(a)] excess in coronary artery disease. Am J Cardiol 1991; 67: 1039-45.

Genest J, McNamara JR, Ordovas JM, Jenner JL, Silberman SR, Anderson KM, Wilson PWF, Salem DN, Schaefer EJ. Lipoprotein cholesterol, apolipoprotein A-I and B and lipoprotein (a) abnormalities in men with premature coronary artery disease. J Am Coll Cardiol 1992; 19: 792802.

Goldberg RJ, Seeley D, Becker RC, Brady P, Chen Z, Osganian V et al. Impact of atrial fibrillation on the in-hospital and long-term survival of patients with acute myocardial infarction: A community-wide perspective. Am Heart J 1990; 119: 996-1001.

Gonzalez-Gronow M, Edelberg JM, Pizzo SV. Further characterization of the cellular plasminogen binding site: evidence that plasminogen 2 and lipoprotein (a) compete for the same site. Biochemistry 1989; 28: 2374-7.

Gottdiener JS, Gay JA, Van-Voorhees LL, DiBianco R, Fletcher RD. Frequency and embolic potential of left ventricular thrombus in dilated cardiomyopathy: assessment by 2-dimensional echocardiography. Am J Cardiol 1983; 52: 1281-5.

Gøtzsche CO, Søgaard P, Ravkilde J, Thygesen K. Effects of captopril on left ventricular systolic and diastolic function after acute myocardial infarction. Am J Cardiol 1992; 70: 15660 .

Greaves M, Pickering C, Knight G, Boulton AJ, Ball J, Ward JD, Preston FE. Changes in the factor VIII complex in diabetic ketoacidosis: evidence of endothelial cell damage? Diabetologia 1987; 30: 160-5.

Green F, Humphries S. Control of plasma fibrinogen levels. Baillieres Clin Haematol 1989; 2: 945-59.

Green KG, Heady A, Oliver MF. Blood pressure, cigarette smoking and heart attack in the WHO co-operative trial of clofibrate. Int J Epidemiol 1989; 18: 355-60.

Griffith GC, Stragnell R, Levinson DC, Moore FJ, Ware AG. Study of beneficial effects of anticoagulant therapy in congestive heart failure. Ann Intern Med 1952; 37:867-87

Grossman W. Diastolic dysfunction and congestive heart failure. Circulation 1990; 81(Suppl III): III-1-III-7.

Grotta JC, Yatsu FM, Pettigrew LC, Rhoades H, Bratina P, Vital D et al. Prediction of carotid stenosis progression by lipid and hematologic measurements. Neurology 1989; 39: 1325-31.

Gurakar A, Hoeg JM, Kostner G, Papadopoulos WM, Brewer HB Jr. Levels of lipoprotein Lp (a) decline with neomycin and niacin treatment. Atherosclerosis 1985; 57: 293-301.

Gurwitz JH, Goldberg RJ, Holden A, Knapic N, Ansell J. Age-related risks of long-term oral anticoagulant therapy. Arch Intern Med 1988; 148: 1733-36.

Gustafsson C, Asplund K, Britton M, Norrving B, Olsson B, Marke LA. Cost-effectiveness of primary stroke prevention in atrial fibrillation: Swedish national perspective. Br Med J 1992; 305: 1457-60.

Gustafsson C, Blomback M, Britton M, Hamsten A, Svensson J. Coagulation factors and the increased risk of stroke in nonvalvular atrial fibrillation. Stroke 1990; 21:47-51. 
Guyton JR, Dahlen GH, Patsch W, Kautz JA, Gotto AM. Relationship of plasma lipoprotein $L p(a)$ levels to race and apolipoprotein B. Arteriosclerosis 1985; 5: 265 - 71.

Hamer A, Peter T, Mandel WJ, Scheinman MM, Weiss D. The potentiation of warfarin anticoagulation by amiodarone.Circulation 1982; 65: 1025-29.

Hamsten A, Blomback M, Wiman B, Svensson J, Szamosi A, de Faire U, Mettinger L. Haemostatic function in myocardial infarction. Br Heart J 1986; 55: 58-66.

Hamsten A, Iselius L, de Faire U, Blomback M. Genetic and cultural inheritance of plasma fibrinogen concentrations. Lancet 1987; 2: 988-90.

Handa K, Kono S, Saku K, Sasaki J, Kawano T, Sasaki Y, Hiroki T, Arakawa K. Plasma fibrinogen levels as an independent indicator of severity of coronary atherosclerosis. Atherosclerosis 1989; 77: $209-13$

Harizi RC, Bianco JA, Alpert JS. Diastolic function of the heart in clinical cardiology. Arch Intern Med 1988; 148: 99- 109

Harker LA. Role of platelets and thrombosis in mechanisms of acute occlusion and restenosis after angioplasty. Am J Cardiol 1987; 60: 20B-28B.

Harrison KA, Haire WD, Pappas AA, Purnell GL, Palmer S, Holdeman KP, Fink LM, Dalrymple GV. Plasma D-dimer: a useful tool for evaluating suspected pulmonary embolus. J Nuclear Med 1993; 34: 896-98.

Hart RG. Cardiogenic embolism to the brain. Lancet 1992; 339: 589-94.

Hatle LK, Appleton CP, Popp RL. Differentiation of constrictive pericarditis and restrictive cardiomyopathy by Doppler echocardiography. Circulation 1989; 79: 357-70.

Haughland JM, Asinger RW, Mikell FL, Elsperger J, Hodges M. Embolic potential of left ventricular thrombi detected by two-dimensional echocardiography. Circulation 1984; 70 : 588-98.

Heinrich J, Sandkamp M, Kokott R, Schulte H, Assmann G. Relationship of lipoprotein (a) to variables of coagulation and fibrinolysis in a healthy population. Clin Chem 1991; 37: 195054.

Heinrich J, Schulte H, Balleisen L, Assmann G, van de Loo J. Predictive value of haemostatic variables in the PROCAM- study. Thromb Haemost. 1991; 65: 815 .

Helmcke F, Nanda NC, Hsiung MC, Soto B, Adey CK, Goyal RG, Gatewood RP. Color Doppler assessment of mitral regurgitation with orthogonal planes. Circulation 1987; 75: 175-83.

Heras M, Sanz G, Betriu A, Magrina J, Perez-Villa F, Paz MA, de Flores T. [Prognostic predictors of left ventricular aneurysm after myocardial infarct.] Rev Esp Cardiol 1992; 45: 238-44.

Hess OM, Felder L, Krayenbuehl HP. Diastolic function in valvular heart disease. Herz 1991; 16: 124-9.

Hesselson AB, Parsonnet V, Bernstein AD, Bonavita GJ. Deleterious effects of long-term single-chamber ventricular pacing in patients with sick sinus syndrome: the hidden benefits of dual-chamber pacing. J Am Coll Cardiol 1992; 19: 1542-9.

Higgins CB, Byrd BF, McNamara MT, Lanzer P, Lipton MJ, Botvinick E, Schiller NB, Crooks LE, Kaufman L. Magnetic Resonance Imaging of the Heart: A review of the experience in 172 subjects. Radiology 1985; 155: 671-79.

Hildebrand F, Cohen BA, Biller J. Cardioembolic Stroke: Part II - Diagnostic Considerations ACC Current Journal Review 1993; 2: 53-54. 
Hinton RC, Kistler JP, Fallon JT, Friedlich AL, Fisher CM. Influence of etiology of atrial fibrillation on incidence of systemic embolism. Am J Cardiol 1977; 40: 509-13.

Hoff HF, Beck GJ, Skibinski Cl, Jürgens G, O'Neil J, Kramer J, Lytle B. Serum Lp(a) level as a predictor of vein graft stenosis after coronary artery bypass surgery in patients. Circulation 1988; 77: 1238-44.

Hofmann T, Kasper W, Meinertz T, Geibel A, Just H. Echocardiographic evaluation of patients with clinically suspected arterial thrombi. Lancet 1990; 336: 1421-24.

Horowitz LN, Spielman SR, Greenspan AM, Mintz GS, Morganroth J, Brown R, Brady PM, Kay HR. Use of amiodarone in the treatment of persistent and paroxysmal atrial fibrillation resistant to quinidine therapy. J Am Coll Cardiol 1985; 6: 1402-7.

Hostmark AT, Bjerkedal T, Kierulf $P$, Flaten $\mathrm{H}$, Ulshagen $\mathrm{K}$. Fish oil and plasma fibrinogen. $\mathrm{Br}$ Med J 1988; 297: 180-1.

Houghton JL, Devlin CW, Besson WT, Crawford W, Fincher RM,Flowers NC, Frank MJ. Possible triggering of paroxysmal atrial fibrillation in normal hearts by psychological stressors: a report of two cases. Am J Med Sci 1990; 300: 234-6.

Houston AB. Doppler ultrasound and the apparently normal heart. Br Heart J 1993; 69: $99-$ 100.

Humphries SE, Cook M, Dubowitz M, Stirling Y, Meade TE. Role of genetic variation at the fibrinogen locus in determination of plasma fibrinogen concentrations. Lancet 1987; 1: 145255.

Hunt BJ. The relation between abnormal hemostatic function and the progression of coronary disease. Curr Opin Cardiol 1990; 5: 758-65.

Hunt FA, Rylatt DB, Hart RA, Bundesen PG. Serum crosslinked fibrin (XDP) and fibrinogen/fibrin degradation products (FDP) in disorders associated with activation of the coagulation or fibrinolytic systems. Br J Haematol 1985; 60: 715-22.

Iga K, Matsuo M, Tsuji A, Nishiki S, Hori K, Matsumura T, Gen H, Tamamura T. [Usefulness of magnetic resonance imaging (MRI) in evaluation of left ventricular apical aneurysm] Kokyu To Junkan 1992; 40: 593-7.

Iga K, Yamashita M, Takahashi S, Tahata T, Miki S, Hori K, Matsumura T, Gen H. [Development of left atrial thrombus after excellent mitral valvuloplasty for chronic severe mitral regurgitation.] Kokyu To Junkan 1993; 41: 183-86.

Ito $\mathrm{M}$, Onodera S, Hashimoto J, Noshiro $\mathrm{H}$, Shinoda S et al.Effect of disopyrimide on initiation of atrial fibrillation and relation to effective refractory period. Am J Cardiol 1989; 63: 561-66.

Jansson $\mathrm{JH}$, Nilsson TK, Johnson $\mathrm{O}$. von Willebrand factor in plasma: a novel risk factor for recurrent myocardial infarction and death. Br Heart J 1991; 66: 351-5.

Jauhiainen $M$, Koskinen $P$, Ehnholm $C$, Frick MH, Manttari M, Manninen V, Huttunen JK. Lipoprotein (a) and coronary heart disease risk : a nested case-control study of the Helsinki Heart Study participants. Atherosclerosis 1991; 89: 59-67.

Jenner JL, Ordovas JM, Lamon-Fava S, Schaefer MM, Wilson PWF, Castelli WP, Schaefer EJ. Effects of age, sex and menopausal status on plasma lipoprotein (a) levels. The Framingham Offspring Study. Circulation 1993; 87: 1135-41.

Jern C, Eriksson E, Tengborn L, Risberg B, Wadenvik H, Jern S. Changes of plasma coagulation and fibrinolysis in response to mental stress. Thromb Haemost 1989; 62: 767-71.

Johannessen KA, Nordrehaug JE, Lippe GVD. Increased occurrence of left ventricular thrombi during early treatment with timolol in patients with acute myocardial infarction. Circulation 1987; 75: 151-55. 
Johnson JA. Diastolic dysfunction in congestive heart failure. Clin Pharm 1991; 10: 850-61.

Jones SL,Close CF, Mattock MB,Jarrett RJ, Keen H, Viberti GC. Plasma lipid and coagulation factor concentrations in insulin dependent diabetics with microalbuminuria. Br Med J 1989; 298: $487-90$.

Judge KW, Pawitan Y, Caldwell J, Gersh BJ, Kennedy JW. Congestive heart failure symptoms in patients with preserved left ventricular systolic function: analysis of the CASS registry. J Am Coll Cardiol 1991; 18: 377-82.

Juul-Möller S, Edvardsson N, Rehnqvist-Ahlberg N. Sotalol versus quinidine for the maintainence of sinus rhythm after direct current conversion of atrial fibrillation. Circulation 1990; 82: 1932-9.

Kannel WB, Abbot RD, Savage DD, McNamara PM. Coronary heart disease and atrial fibrillation: the Framingham Study. Am Heart J. 1983; 106: 389-96.

Kannel WB, D'Agostino RB, Belanger AJ. Fibrinogen, cigarette smoking, and risk of cardiovascular disease: Insights from the Framingham study. Am Heart J 1987; 113: 1006 10

Kannel WB, D'Agostino RB, Wilson PWF, Belanger AJ, Gagnon DR. Diabetes, fibrinogen and risk of cardiovascular disease: the Framingham experience. Am Heart J 1990; 120: 672-76.

Kannel WB, Wolf PA, Castelli WP, D'Agostino RB. Fibrinogen and risk of cardiovascular disease. The Framingham Study. JAMA 1987; 258: 1183-86.

Kannell WB, Abbott RD, Savage DD, McNamara PM. Epidemiological features of chronic atrial fibrillation. The Framingham Study. N Engl J Med 1982; 306: 1018-22.

Kapila A, Hart R. Calcific cerebral emboli and aortic stenosis: detection of computed tomography. Stroke 1986; 17: 619-21.

Karadi I, Kostner GM, Gries A, Nimpf J, Romics L, Malle E. Lipoprotein (a) and plasminogen are immunochemically related. Biochem Biophys Acta 1988; 960: 91-97.

Kato R, Ikeda N, Yabek SM, Kannan R, Singh BN. Electrophysiologic effects of the levo- and dextrorotatory isomers of sotalol in isolated cardiac muscle and their in vivo pharmacokinetics. J Am Coll Cardiol 1986; 7: 116-125.

Kaufman DW, Palmer JR, Rosenberg L, Shapiro S. Cigar and pipe smoking and myocardial infarction in young men. Br Med J 1987; 294: 1315-16.

Keating EC, Gross SA, Schlamowitz RA, Glassman J, Mazur JH, Pitt WA, Miller D. Mural thrombi in myocardial infarctions. Am J Med 1983; 74: 989-95.

Kessler C, Henningsen H, Reuther R, Kinnig B, Rösch M. Identification of intracardiac thrombi in stroke patients with indium-111 platelet scintigraphy. Stroke 1987; 18: 63-67.

Kessler KM. Predictors of left ventricular thrombus formation: flow, asynergy and morphology. J Am Coll Cardiol 1990; 15: 361-62.

Khaja F, Parker JO. Hemodynamic effects of cardioversion in chronic atrial fibrillation. Arch Intern Med 1972; 129: 433-40.

Klein HO, Kaplinsky E Verapamil and digoxin: their respective effects on atrial fibrillation and their interaction. Am J Cardiol 1982; 50: 894-902.

Knecht MF, Heinrich F, Spanuth E. Evaluation of plasma D-dimer in the diagnosis and in the course of fibrinolytic therapy of deep venous thrombosis and pulmonary embolism. Thromb Res 1992; 67: 213-20. 
Koenig A, Theolade R, Chauvin M, Brechenmacher C. [Embolic complications of chronic atrial fibrillation.] Arch Mal Coeur Vaiss 1992; 85: 315-23.

Kondili A, Kastrati A, Popa Y. Comparative evaluation of verapamil, flecainide and propafenone for the acute conversion of atrial fibrillation to sinus rhythm. Wien Klin Wochenschr. 1990;102:510-3.

Kopecky SL, Gersh BJ, McGoon MD et al. The natural history of lone atrial fibrillation. A population-based study over three decades. N Engl J Med 1987 317: 669-74.

Koppensteiner R, Moritz A, Schlick W, Fenzl G, Roedler S, Ehringer H, Wollner E. Blood rheology after cardiac valve replacement with mechanical prostheses or bioprostheses. Am J Cardiol 1991; 67: 79-83.

Korsan-Bengtsen K, Wilhelmsen L, Tibblin G. Blood coagulation and fibrinolysis in a random sample of 788 men 54 years old: 'Il.Relations of the variables to 'risk factors' for myocardial infarction. Thromb Diathes Haemorrh 1972; 28: 99-108.

Kumagai K, Fukunami $M$, Ohmori $M$, Kitabatake $A$, Kamada $T$, Hoki N. Increased intracardiovascular clotting in patients with chronic atrial fibrillation. J Am Coll Cardiol 1990; 16: 377-80.

Kutner M, Nixon G, Silverstone F. Physicians' attitudes toward oral anticoagulants and antiplatelet agents for stroke prevention in elderly patients with atrial fibrillatiuon. Arch Intern Med 1991; 151: 1950-53.

Kweider M, Lowe GDO, Murray GD, Kinane DF, McGowan DA. Dental disease, fibrinogen and white cell count; links with myocardial infarction? Scot Med J 1993; 38: 73-74.

La Rovere MT, Pozzoli M, Traversi E, Opasich C, Specchia G. Exercise tolerance in patients with and without ventricular aneurysm. Cardiology 1987; 74: 483-9.

Labovitz AJ, Pearson AC. Evaluation of left ventricular diastolic function: clinical relevance and recent Doppler echocardiographic insights. Am Heart J 1987; 114: 836- 51.

Lancaster TR, Singer DE, Sheehan MA, Oertel LB, Maraventano SW, Hughes RA, Kistler P. The impact of long-term warfarin therapy on quality of life. Evidence from a randomized trial. Boston Area Anticoagulation Trial for Atrial Fibrillation Investigators. Arch Intern Med 1991; 151: $1944-49$.

Lapeyre AC, Steele PM, Kazmier FJ, Vlietstra RE, Fuster V. Systemic embolism in chronic left ventricular aneurysm: incidence and the role of anticoagulation. J Am Coll Cardiol 1985; 6: 534-8.

Lawn RM. Lipoprotein (a) in heart disease. Sci Am 1992; 266: 54-60.

Lee AJ, Lowe GDO, Woodward M, Tunstall-Pedoe H. Fibrinogen in relation to personal history of prevalent hypertension, diabetes, stroke, intermittent claudication, coronary artery disease, and family history: the Scottish Heart Health Study. Br Heart J 1993; 69: 338-42.

Lee AJ, Smith WC, Lowe GD, Tunstall-Pedoe H. Plasma fibrinogen and coronary risk factors: the Scottish Heart Health Study. J Clin Epidemiol 1990; 43: 913-19.

Leitch JW, Klein G, Yee R, Guiraudon G. Sinus-node-atrioventricular node isolation: Iong term results with the "corridor" operation for atrial fibrillation. J Am Coll Cardiol 1991; 17: 970-5.

Leschke $M$, Blanke $H$, Stellwaag $M$, Motz $W$, Strauer BE. (Hyperfibrinogenemia and pathological plasma viscosity. Pathogenetic factors in unstable angina pectoris?) Dtsch Med Wochenschr 1988; 113: 1175-81.

Leschke M, Motz W, Blanke H, Strauer BE. Blood rheology in hypertension and hypertensive heart disease. J Cardiovasc Pharmacol 1987; 10 Suppl 6; S103-10. 
Leschke M, Vogt M, Motz W, Strauer BE. Blood rheology as a contributing factor in reduced coronary reserve in systemic hypertension. Am J Cardiol 1990; 65: 56G-59G.

Levine MN, Raskob G, Hirsh J. Hemorrhagic complications of long-term anticoagulant therapy. Chest 1990; 95 (suppl): 26S-36S.

Levy S. Direct current cardioversion of established atrial fibrillation. Clin Cardiol 1992; 15 : 445-49.

Lewis RP. Cerebral embolism in mitral valve prolapse. In: Boudoulas H, Wooley CF (eds). Mitral valve prolapse and the mitral valve prolapse syndrome. Mount Kisco, NY: Futura Publishing, 1988: 289-98.

Lidegaard $\varnothing$. Oral contraception and risk of a cerebral thromboembolic attack: results of a case-controlled study. Br Med J 1993; 306: 956-63.

Lin SL, Fisher MJ, Tak T, Rahimtoola SH, Chandraratna PA. Platelet activation in patients with mitral valve prolapse. Can J Cardiol 1989; 5: 84-6.

Lindroos M, Kupari M, Heikkilä J, Tilvis R. Prevalence of aortic valve abnormalities in the elderly: An echocardiographic study of a random populatiom sample. J Am Coll Cardiol 1993; 21: $1220-5$.

Lipkin DP, Frenneaux M, Stewart R, Joshi J, Lowe T, McKenna WJ. Delayed improvement in exercise capacity after cardioversion of atrial fibrillation to sinus rhythm. $\mathrm{Br}$ Heart $\mathrm{J} 1988 ; 59$ : 572-77.

Little WC, Downes TR. Clinical evaluation of left ventricular diastolic performance. Prog Cardiovasc Dis 1990; 32: 273- 90

Loscalzo J, Wienfeld M, Fless GM, Scanu AM. Lipoprotein (a), fibrin binding and plasminogen activation. Arteriosclerosis 1990; 10: 240-45.

Lowe GDO, Drummond MM, Forbes CD, Barbenel JC. Increased blood viscosity in young women using oral contraceptives.Am J Obstet Gynecol 1980; 137: 840-42.

Lowe GDO, Drummond MM, Lorimer AR, Hutton I, Forbes CD, Prentice CRM, Barbenel JC. Relation between extent of coronary artery disease and blood viscosity. Br Med J 1980; 280: 673-74.

Lowe GDO, Drummond MM, Third JHLC, Bremner WF, Forbes CD, Prentice CRM, Lawrie TDV. Increased plasma fibrinogen and platelet aggregates in Type II Hyperlipoproteinaemia. Thromb Haemost 1979; 42: 1503-7.

Lowe GDO, Fowkes FG, Dawes J, Donnan PT, Lennie SE, Housley E. Blood viscosity, fibrinogen and activation of coagulation and leukocytes in peripheral arterial disease and the normal population in the Edinburgh Artery Study. Circulation 1993; 87: 1915-20.

Lowe GDO, Jaap AJ, Forbes CD. Relation of atrial fibrillation and high haematocrit to mortality in acute stroke. Lancet 1983; 1: 784-6.

Lowe GDO, Lee AJ, Rumley A, Smith WCS, Tunstall-Pedoe H. Epidemiology of haematocrit, white cell count, red cell aggregation and fibrinogen: The Glasgow MONICA Study. Clin Hemorheol 1992; 12: 535-44.

Lowe GDO. Antithrombotic treatment and atrial fibrillation. Br Med J 1992; 305: 1445-46.

Lowe GDO. Blood rheology in arterial disease. Clin Sci 1986; 71: 137-46.

Lown B. Electrical reversion of cardiac arrhythmias. Br Heart J 1967; 29: 469-89.

Lundstrőm T, Rydén L. Haemorrhagic and thromboembolic complications in patients with atrial fibrillation on anticoagulant prophylaxis. J Intern Med 1989; 225: 137-42. 
Lynch JJ, Coskey LA, Montgomery DG, Lucchesi BR. Prevention of ventricular fibrillation by dextrorotatory sotalol in a conscious canine model of sudden coronary death. Am Heart $\mathrm{J}$ 1985; 109: 949-58.

Machin SJ, Mackie IJ. Routine measurement of fibrinogen concentration. Br Med J 1993; 307: 882-83.

Maeda S, Abe A, Seishima M, Makino K, Noma A, Kawade M. Transient change of serum lipoprotein (a) as an acute phase protein. Atherosclerosis 1989; 78: 145-50.

Mahmarian JJ, Pratt CM. Silent myocardial ischemia in patients with coronary artery disease. Circulation 1990; 81 (suppl III): III-33 - III-40.

Malaia LT, Volkov VI, Topchii II, Dykun laV, Shuliak LN. (The content of fibrionectin, fibrinogen, its degradation products and myoglobin in patients with ischaemic heart disease.) Ter Arkh 1990; 62: 87-90.

Mancini GBJ, Goldberger AL. Cardioversion of atrial fibrillation: consideration of embolization, anticoagulation, prophylactic pacemaker and long term success. Am Heart J 1982; 104: 61721.

Manning WJ, Leeman DE, Gotch PJ, Come PC. Pulsed Doppler evaluation of atrial mechanical function after electrical cardioversion of atrial fibrillation. J Am Coil Cardiol 1989; 13: $617-23$.

Manning WJ, Silverman DI, Gordon SPF, Krumholz HM, Douglas PS. Cardioversion from atrial fibrillation without prolonged anticoagulation with use of transesophageal echocardiography to exclude the presence of atrial thrombi. N Engl J Med 1993; 328: 750-5.

Manning WJ, Silverman DI, Katz SE, Douglas PS. Atrial ejection force: A noninvasive assesment of atrial systolic function. J Am Coll Cardiol 1993; 22: 221-25.

Marcus ML, Dellsperger KC. Determinants of systolic and diastolic ventricular function. In: Marcus ML, Schelbert HR, Skorton DJ, Wolf GL (eds) Cardiac Imaging: A Companion to Braunwald's Heart Disease. Philadelphia: WB Saunders.1991; 24-38.

Mares M, Bertolo C, Terribile, Girolami A. Hemorheological study in patients with coronary artery disease. Cardiology 1991; 78: 111-116.

Martin JF, Bath PMW, Burr ML. Influence of platelet size on outcome after myocardial infarction. Lancet 1991; 338: 1409-11.

Masarei JRL, Rouse H, Lynch WJ, Robertson K, Vandongen R, Beilin LJ. Effects of a lactoovo vegetarian diet on serum concentrations of cholesterol, triglyceride, $\mathrm{HDL}-\mathrm{C}, \mathrm{HDL}_{2}-\mathrm{C}$, $\mathrm{HDL}_{3}-\mathrm{C}$, apolipoprotein-B, and Lp(a). Am J Clin Nutr 1984; 40: 468-79.

Maze SS, Kotler MN, Parry WR. Flow characteristics in the dilated left ventricle with thrombus: qualitative and quantitative Doppler analysis. J Am Coll Cardiol 1989; 13: 873-81.

Meade TW, Chakrabarti R, Haines AP, North WRS, Stirling Y. Characteristics affecting fibrinolytic activity and plasma fibrinogen concentrations. Br Med J 1979; i: 153-56.

Meade TW, Dyer S, Howarth DJ, Imeson JD, Stirling Y. Antithrombin III and procoagulant activity: sex differences and effects of the menopause. Br J Haematol 1990; 74: 77-81.

Meade TW, Haines AP, Imeson JD, Stirling Y, Thompson SG. Menopausal status and haemostatic variables. Lancet 1983;1: 22-24.

Meade TW, Imeson J, Stirling Y. Effects of changes in smoking and other characteristics on clotting factors and the risk of ischaemic heart disease. Lancet 1987; 2: 986-88. 
Meade TW, Mellows S, Brozovic M, Miller GJ, Chakrabarti RR, North WR et al. Haemostatic function and ischaemic heart disease: principal results of the Northwick Park heart study. Lancet 1986; 2: 533-7.

Meade TW, North WRS, Chakrabarti R, Stirling Y, Haines AP, Thompson SG. Haemostatic function and cardiovascular death: early results of a prospective study. Lancet 1980; 1: 10504.

Meltzer RS, Visser CA, Fuster V. Intracardiac thrombi and systemic embolism. Ann Intern Med 1986; 104: 689-98.

Memeh CU. The relationship betwen body weight and plasma viscosity in hypertensive diabetic Nigerians. J Hypertens 1990; 8: 711-14.

Mercuri M, Orecchini G, Susta A, Tazza D, Ciuffetti G. Correlation between hemorheologic parameters and carotid atherosclerosis in stroke. Angiology 1989; 40:283-86.

Merino A, Hauptman P, Badimon L, Badimon JJ, Cohen M, Fuster V, Goldman M. Echocardiographic 'smoke' is produced by an interaction of erythrocytes and plasma proteins modulated by shear forces. J Am Coll Cardiol 1992; 20: 1661 - 8.

Metcalfe MJ, Smith F, Jennings $K$, Paterson $M$. Does cardioversion of atrial fibrillation result in myocardial damage? Br Med J 1988; 296: 1364.

Mettinger KL. A study of hemostasis in ischemic cerebrovascular disease I.Abnormalities in factor VIII and antithrombin. Thromb Res 1982; 26: 183-92.

Middlekauff HR, Stevenson WG, Stevenson LW. Prognostic significance of atrial fibrillation in advanced heart failure. A study of 390 patients. Circulation 1991; 84: 40-8.

Mikell FL, Asinger RW, Elsperger $\mathrm{KJ}$, Anderson WR, Hodges M. Regional stasis of blood in the dysfunctional left ventricle: Echocardiographic detection and differentiation from early thrombosis. Circulation 1982; 66: 755-63.

Miles LA, Fless GM, Levin EG, Scanu AM, Plow EF. A potential basis for the thrombotic risks associated with lipoprotein (a). Nature 1989; 339: 301-3.

Moisseev SI. (The role of hemostasis and rheologic properties of blood in stable and progressive exertion-induced sternocardia.) Kardiologiia 1988; 28: 67-71.

Møller L, Kristensen TS. Plasma fibrinogen and ischemic heart disease risk factors. Arterioscler Thromb 1991; 11: 344-50.

Moore RD, Pearson TA. Moderate alcohol consumption and coronary artery disease: A review. Medicine (Baltimore) 1986; 65: 242-67.

Mullen DC, Posey L, Gabriel R, Singh HM, Flemma RJ, Lepley D. Prognostic considerations in the management of left ventricular aneurysms. Ann Thorac Surg 1977; 23: 455-60.

Nair CK, Sketch MH, Mahoney PD, Lynch JD, Mooss AN, Kenney NP. Detection of left ventricular thrombi by computerised tomography. Br Heart J 1981; 45: 535-41.

Naito M, Dreifus LS, Mardelli TJ, Chien CC, David D et al. Echocardiographic features of atrioventricular and ventriculoatrial conduction. Am J Cardiol 1980; 46: 625-33.

Nathan AW, Hellestrand KJ, Bexton RS, Banim SO, Spurrell RAJ, Camm AJ. Proarrhythmic effects of the new antiarrhythmic effects agent flecainide acetate. Am Heart J 1984; 107: 22228.

Nichols AB, Owen J, Kaplan KL, Sciacca RR, Cannon PJ, Nossel HL. Fibrinopeptide A, platelet factor 4, and beta-thromboglobulin levels in coronary heart disease. Blood 1982; 60: $650-4$. 
Nishide $M$, Irino T, Gotoh M, Naka M, Tsuji $K$. Cardiac abnormalities in ischemic cerebrovascular disease studied by two-dimensional echocardiography. Stroke 1983; 14: 541-45.

Nishimura RA, Abel MD, Hatle LK, Tajik AJ. Assessment of diastolic function of the heart: background and current applications of Doppler echocardiography. Part II. Clinical studies. Mayo Clin Proc 1989; 64: 181-204.

Nishino M, Ito T, Yasuno M, Kuryu T, Yamada Y, Abe H, Hori M, Kamada T. Serum lipoprotein (a) as a risk factor for thoracic aortic atherosclerosis in subjects aged $>40$ years. Am J Cardiol 1993; 72: 227-29.

Nixon JV. Left ventricular mural thrombus. Arch Intern Med 1983; 143: 1567-71.

Noc M, Stajer D, Horvat $M$. Intravenous amiodarone versus verapamil for acute conversion of paroxysmal atrial fibrillation to sinus rhythm. Am J Cardiol 1990; 65: 679-80.

O'Neill PG, Puleo PR, Bolli R, Rokey R. Return of atrial mechanical function following electrical cardioversion of atrial arrhythmias. Am Heart J 1990; 120: 353-59.

Obarski TP, Salcedo EE, Castle LW, Stewart WJ. Spontaneous echo contrast in the left atrium during paroxysmal atrial fibrillation. Am Heart J 1990; 120: 988-90.

Oh JK, Hatle LK, Sinak LJ, Seward JB, Tajik AJ. Characteristic Doppler echocardiographic pattern of mitral inflow velocity in severe aortic regurgitation. J Am Coll Cardiol 1989; 14: 1712-17.

Ohe $T$, Shimomura $K$, Inagaki $M$. The effects of cycle length on the fragmented atrial activity zone in patients with sick sinus syndrome. J Electrocardiol 1987; 20: 364-68.

Olsson SB, Orndahl G, Ernestrom $S$ et al. Spontaneous reversion from long lasting atrial fibrillation to sinus rhythm. Acta Med Scand. 1980; 207: 5-20.

Packer DL, Bardy GH, Worley SJ, Smith MS, Cobb FR, Coleman RE, Gallagher JJ, German LD. Tachycardia-induced cardiomyopathy: A reversible form of left ventricular dysfunction. Am $\mathbf{J}$ Cardiol 1986; 57: 563- 70.

Packer M. Abnormalities of diastolic function as a potential cause of exercise intolerance in chronic heart failure. Circulation 1990; 81 (suppl III):III-78-III-86.

Parameshwar J, Poole-Wilson PA, Sutton GC. Heart failure in a district general hospital. J R Coll Physicians Lond 1992; 26: 139-42.

Parra HJ, Mezdour H, Cachera C, Dracon M, Tacquet A, Fruchart JC. Lp(a) lipoprotein in patients with chronic renal failure treated by hemodialysis. Clin Chem 1987; 33: 721.

Pasternak RC, Braunwald E, Sobel BE. Acute Myocardial Infarction. In: Braunwald E (ed) Heart Disease. A Textbook of Cardiovascular Medicine. Philadelphia: 4th ed. WB Saunders. 1992; 1200-1291.

Pedro-Botet J, Senti M, Nogués X, Rubiés-Prat J, Roquer J, D'Olhaberriague L, Olivé J. Lipoprotein and apolipoprotein profile in men with ischemic stroke. Stroke 1992; 23: 155662.

Penny WF, Weinstein M, Salzman EW, Ware JA. Correlation of circulating von Willebrand factor levels with cardiovascular hemodynamics. Circulation 1991; 83: 1630-6.

Perski A, Olsson G, Landou C, de Faire U, Theorell T, Hamsten A. Minimum heart rate and coronary atherosclerosis: independent relations to global severity and rate of progression of angiographic lesions in men with myocardial infarction at a young age. Am Heart J 1992; 123: 609-16. 
Petersen P, Boysen G, Godttredsen J, Andersen ED, Andersen B. Placebo controlled, randomised trial of warfarin and aspirin for prevention of thromboembolic complications in chronic atrial fibrillation: the Copenhagen AFASAK study. Lancet 1989; 1: 175-9.

Petersen P, Godtfredsen J. Embolic complications in paroxysmal atrial fibrillation. Stroke 1986; 17: 622-26.

Petersen P, Kastrup J, Brinch K, Godttredsen J, Boysen G. Relation between left atrial dimension and duration of atrial fibrillation. Am J Cardiol 1987; 60: 382-84.

Petersen P, Kastrup J, Wilhelmsen R, Schutten HJ. Atrial natriuretic peptide in atrial fibrillation before and after electrical cardioversion therapy.Eur Heart J 1988;9:639-41.

Petersen P, Pedersen F, Johnsen A, Madsen EB, Brun B, Boysen G, Godtfredsen J. Cerebral computed tomography in paroxysmal atrial fibrillation. Acta Neurol Scand 1989; 79: 482-6.

Petersen P. Thromboembolic complications in atrial fibrillation. Stroke 1990; 21: 4-13.

Petersen P.Thromboembolic complications of atrial fibrillation and their prevention: A review. Am J Cardiol 1990; 65: 24C-28C.

Petrin TJ, Tavel ME. Idiopathic hypertrophic subaortic stenosis as observed in a large community hospital: relation to age and history of hypertension. J Am Geriatr Soc 1979; 27: 43-46.

Pietersen $\mathrm{AH}$, Hellemann $\mathrm{H}$. Usefulness of flecainide for prevention of paroxysmal atrial fibrillation and flutter. Danish-Norwegian Flecainide Mutticenter Study Group. Am J Cardiol 1991; 67: 713-7.

Platia EV, Michelson EL, Porterfield JK, Das G. Esmolol versus verapamil in the acute treatment of atrial fibrillation or atrial flutter. Am J Cardiol 1989; 63: 925-29.

Pollak H, Fischer M, Fritsch S, Enenkel W. Are admission plasma fibrinogen levels useful in the characterization of risk groups after myocardial infarction treated with fibrinolysis? Thromb Haemost 1991; 66 : 406-9.

Pouleur $\mathrm{H}$, Hanet $\mathrm{C}$, Gurne O, Rousseau MF. Focus on diastolic dysfunction: a new approach to heart failure therapy. Br J Clin Pharmacol 1989; 28 Suppl 1: 41S-52S.

Pratt CM, Brater DC, Harrell FE et al. Clinical and regulatory implications of the Cardiac Arrhythmia Suppression Trial. Am J Cardiol 1990; 65: 103-5.

Pritchett EL, McCarthy EA, Wilkinson WE. Propafenone treatment of symptomatic paroxysmal supraventricular arrhythmias. A randomized, placebo-controlled, crossover trial in patients tolerating oral therapy. Ann Intern Med 1991; 114: 539-44.

Pritchett ELC. Management of atrial fibrillation. N Engl J Med 1992; 326: 1264-71.

Qiu SQ, Theroux P, Genest J Jr, Solymoss BC, Robitaille D, Marcil M. Lipoprotein (a) blood levels in unstable angina pectoris, acute myocardial infarction, and after thrombolytic therapy. Am J Cardiol 1991; 67: 1175-9.

Qizilbash N, Jones L, Warlow C, Mann J. Fibrinogen and lipid concentrations as risk factors for transient ischaemic attacks and minor ischaemic strokes.Br Med J 1991; 303: 605-9.

Radack K, Deck C, Huster G. Dietary supplementation with low-dose fish oils lowers fibrinogen levels: A randomized, double-blind controlled study. Ann Intern Med 1989; 111: 757-58.

Ramirez LC, Arauz-Pacheco C, Lackner C, Albright G, Adams BV, Raskin P. Lipoprotein (a) levels in diabetes mellitus: relationship to metabolic control. Ann Intern Med 1992; 117: 4247. 
Ramsay LE. Warfarin in chronic atrial fibrillation. Lancet 1993; 341: 1376-77.

Rancurel G. Marelle L, Vincent D, Catala, Arzimanoglou A, Vacheron A. Spontaneous calcific cerebral embolus from a calcific aortic stenosis in a middle cerebral artery infarct. Stroke 1989; 20: 691-93.

Rawles JM, Metcalfe MJ, Jennings $\mathrm{K}$. Time of occurence, duration, and ventricular rate of paroxysmal atrial fibrillation: the effect of digoxin. Br Heart J 1990; 63: 225-7.

Rebello R, Brownlee WC. Intermittent ventricular standstill during chronic atrial fibrillation in patients with dizziness or syncope. PACE 1987; 10: 1271-76.

Reeder GS, Lengyel M, Tajik AJ, Seward JB, Smith HC, Danielson GK. Mural thrombus in left ventricular aneurysm: Incidence, role of angiography, and relation between anticoagulation and embolization. Mayo Clin Proc 1981; 56: 77-81.

Rees A. Lipoprotein (a): a possible link between lipoprotein metabolism and thrombosis. $\mathrm{Br}$ Heart J 1991; 65: 2-3.

Reimold SC, Cantillon CO, Friedman PL, Antman EM. Propafenone versus sotalol for supression of recurrent symptomatic atrial fibrillation. Am J Cardiol 1993; 71: 558-563.

Repique LJ, Shah SN, Marais GE. Atrial fibrillation 1992. Management strategies in flux. Chest 1992; 101: 1095-1103.

Ritchie DG, Levy BA, Adams MA, Fuller GM. Regulation of fibrinogen synthesis by plasminderived fragments of fibrinogen and fibrin: an indirect feedback pathway. Proc Natl Acad Sci USA 1982; 79: 1530-34.

Rokey R, Kuo LC, Zoghbi WA, Limacher MC, Quinones MA. Determination of parameters of left ventricular diastolic filling with pulsed Doppler echocardiography: comparison with cineangiography. Circulation 1985; 71: 543-50.

Rokitansky CV. Über einige der eichtigsten Krankheiten. K.u.K. Hof-und Staatsdruckerei, Wien 1852.

Rosengren A, Wilhelmsen L, Eriksson E, Risberg B, Wedel H, Lipoprotein (a) and coronary heart disease: a prospective case-control study in a general population sample of middle aged men. Br Med J 1990; 301: 1248-51.

Rosengren A, Wilhelmsen L, Welin L, Tsipogianni, Teger-Nilsson AC, Wedel $H$. Social influences and cardiovascular risk factors as determinants of plasma fibrinogen concentration in a general population sample of middle aged men. Br Med J 1990; 300: 634-8.

Rosenqvist $M$, Brandt $J$, Schuller $H$. Long-term pacing in sinus node disease: effects of stimulation mode on cardiovascular morbidity and mortality. Am Heart J 1988; 166: 16-22.

Rousseau MF, Pouleur H, Debaisieux JC, Van Eyll C, Charlier AA. Contrasting effects of single doses of pindolol and xamoterol on left ventricular diastolic dysfunction. $\mathrm{Br} \mathrm{J}$ Clin Pharmacol 1989; 28 Suppl 1; 84S-85S.

Rouy D, Grailhe P, Nigon F, Chapman J, Anglés-Cano E. Lipoprotein (a) impairs generation of plasmin by fibrin-bound tissue-type plasminogen activator. Invitro studies in a plasma milieu. Arteriosclerosis Thromb 1991; 11:629-38.

Roy D, Marchand E, Gagné P, Chabot M, Cartier R. Usefulness of anticoagulant therapy in the prevention of embolic complications of atrial fibrillation. Am Heart J 1986; 112: 1039-43.

Royal College of General Practitioners' Oral Contraception Study. Mortality among oralcontraceptive users. Lancet 1977; 2:727-31. 
Sage JI, Van Uitert RL. Risk of recurrent stroke in patients with atrial fibrillation and nonvalvular heart disease. Stroke 1983; 14: 537-40.

Sahar DI, Reiffel JA, Bigger JT, Squatrito A, Kidwell GA Efficacy, safety, and tolerance of dsotalol in patients with refractory supraventricular tachyarrhythmias. Am Heart J 1989; 117: 562-68.

Sahn DJ, DeMaria A, Kisslo J, Weyman A. Recommendations regarding quantitation in MMode echocardiography: Results of a survey of echocardiographic measurements. Circulation 1978;58:1072-83.

Sandercock P, Bamford J, Dennis M, Burn J, Slattery J, Jones L, Boonyakarnkul S, Warlow C. Atrial fibrillation and stroke: prevalence in different types of stroke and influence on early and long term prognosis (Oxfordshire community stroke project) Br Med J 1992; 305: 1460-5.

Sandholzer C, Boerwinkle E, Saha N, Tong MC, Utermann G. Apolipoprotein (a) phenotypes, $L p$ (a) concentrations and plasma lipid levels in relation to coronary artery disease in a Chinese population: evidence for the role of the apo(a) gene in coronary artery disease. J Clin Invest 1992; 89: 1040-46.

Sandkamp M, Funke H, Schulte M, Kohler E, Assmann G. Lipoprotein (a) is an independent risk factor for myocardial infarction at a young age. Clin Chem 1990; 36: 20-3.

Sanfilippo AJ, Abascal VM, Sheehan M, Oertel LB, Harrigan P, Hughes RA, Weyman AE. Atrial enlargement as a consequence of atrial fibrillation. A prospective echocardiographic study. Circulation 1990; 82: 792-97.

Saour JN, Sieck JO, Mamo LA, Gallus AS. Trial of different intensities of anticoagulation in patients with prosthetic heart valves. N Engl J Med 1990; 322: 428-32.

Scanu AM. Lipoprotein (a). A genetic risk factor for premature coronary heart disease. JAMA 1992; 267: 3326-29.

Schlichter J, Hellerstein HK, Katz LN. Aneurysm of the heart: a correlative study of one hundred and two proved cases. Medicine (Baltimore) 1954; 33: 43-86.

Schmitz-Huebner U, Thompson SG, Balleisen L, Fechtrup C, Grosse-Heitmeyer W, Kirchhof $B$, Most E, et al. Lack of association between haemostatic variables and the presence or the extent of coronary atherosclerosis.Br Heart J 1988; 59: 287-91.

Scott J Lipoprotein (a). Br Med J 1991; 303: 663-34.

Seed M, Hoppichler F, Reaveley D, et al. Relation of serum lipoprotein (a) concentration and apolipoprotein (a) phenotype to coronary heart disease in patients with familial hypercholesterolemia. N Engl J Med 1990; 322: 1494-9.

Selzer A. Atrial fibrillation revisited. N Engl J Med 1982; 306: 1044-45.

Setaro JF, Soufer R, Remetz MS, Perlmutter RA, Zaret BL. Long-term outcome in patients with congestive heart failure and intact systolic left ventricular performance. Am $\mathrm{J}$ Cardiol 1992; 69: 1212-16.

Shaikh MA, Lavine SJ. Effect of mitral regurgitation on diastolic filling with left ventricular hypertrophy. Am J Cardiol 1988; 61: 590-94.

Shearman RP. Contraception and sterilization. In: Whitfield CR (ed). Dewhurst's Textbook of Obstetrics and Gynaecology for Postgraduates. 4th ed. Oxford: Blackwell Scientific Publications. 1986: 568-579.

Shen WF, Tribouilloy C, Mirode A, Dufosse H, Lesbre JP. Left ventricular aneurysm and prognosis in patients with first acute transmural anterior myocardial infarction and isolated left anterior descending artery disease. Eur Heart J 1992; 13: 39-44. 
Shepherd RF, Zachariah PK, Shub C. Hypertension and left ventricular diastolic function. Mayo Clin Proc 1989; 64: 1521-32.

Sherman DG, Dyken ML, Fisher M, Harrison MJG, Hart RG. Antithrombotic therapy for cerebrovascular disorders. Chest 1990; 95 (suppl): 140S-155S.

Shihabi ZK, Konen JC, McCormick CP.Plasma fibrinogen in Type II diabetics. Clin Physiol Biochem 1990; 8: 297-300.

Shimomura K, Ohe T, Uehara S, Matsuhisa M, Kamakura S, Sato I. Significance of atrial fibrillation as a precursor of embolism. Am J Cardiol 1989; 63: 1405-7.

Shintani S, Kikuchi S, Hamaguchi $H$, Shiigai T. High serum lipoprotein (a) levels are an independent risk factor for cerebral infarction. Stroke 1993; 24: 965-69.

Sigel B, Coelho JCU, Spigos DG, Flanigan DP, Schuler JJ, Kasprisin DO, Nyhus LM, Capek V. Ultrasonography of blood during stasis and coagulation. Invest Radiol 1981; 16: 71-76.

Simpson HCR, Mann JI, Meade TW, Chakrabarti R, Stirling Y, Woolf L. Hypertiglyceridaemia and hypercoagulability. Lancet 1983; 1: 786-89.

Simpson MT, Oberman A, Kouchoukos NT, Rogers WJ. Prevalance of mural thrombi and systemic embolization with left ventricular aneurysm: effect of anticoagulation therapy. Chest 1980; 77: 463-9.

Singer DE. Randomized trials of warfarin for atrial fibrillation. N Engl J Med 1992; 327: 145153.

Singh BN, Opie LH, Marcus FI. Antiarrhythmic Agents. In: Opie LH (ed) Drugs for the heart. Philadelphia. WB Saunders. 3rd ed. 1991; 180-216.

Slunga L, Johnson O, Dahlén GH, Eriksson S. Lipoprotein (a) and acute phase proteins in acute myocardial infarction. Scand J Clin Lab Invest 1992; 52: 95-101.

Small M, Lowe GD, Beattie JM, Hutton I, Lorimer AR, Forbes CD. Severity of coronary artery disease and basal fibrinolysis. Haemostasis 1987; 17: 305-11.

Smith EB, Keen GA, Grant A, Stirk C. Fate of fibrinogen in human arterial intima. Arteriosclerosis 1990; 10: 263-75.

Solerte SB, Fioravanti M, Schifino N, Patti AL, Gamba G, Ferrari E. Hemorheologic and hemostatic changes in long-standing insulin-dependent (type I) diabetic patients with peripheral and autonomic cardiovascular neuropathy. Acta Diabetol Lat 1988; 25: 235-42.

Sonkodi S, Rednik A, Kovács A. Correlations of the fibrinogen level with the blood pressure and with the plasma renin activity in hypertensive patients. Cor Vasa 1979; 21: 228-32.

Sonnhag C, Kallryd A, Nylander E, Ryden L. Long-term efficacy of flecainide in paroxysmal atrial fibrillation. Acta Med Scand 1988; 224: 563-69.

Sosa-Suarez G, Lampert S, Graboys TB, Ravid S, Lown B. Changes in left atrial size due to chronic atrial fibrillation. J Am Coll Cardiol 1989; 13: 206A.

Soufer R, Wohlgelernter, Vita NA, Amuchestegui M, Sostman HD, Berger HJ, Zaret BL. Intact systolic left ventricular function in clinical congestive heart failure. Am J Cardiol 1985;55: 1032-36.

Spinale FG, Tomita M, Zellner JL, Cook JC, Crawford FA, Zile MR. Collagen remodelling and changes in LV function during development and recovery from supraventricular tachycardia. Am J Physiol1991; 261: H308-18. 
Spirito P, Maron BJ, Bonow RO. Noninvasive assessment of left ventricular diastolic function: Comparative analysis of Doppler echocardiographic and radionuclide techniques. J Am Coll Cardiol 1986; 7: 518- 26.

Srinivasan SR, Dahlen GH, Jarpa RA, Webber LS, Berenson GS, Racial (black-white) differences in serum lipoprotein (a) distribution and its relation to parental myocardial infarction in children. Bogalusa Heart Study. Circulation 1991; 84: 160-7.

St Goar FG, Masuyama T, Alderman EL, Popp RL. Left ventricular diastolic dysfunction in end-stage dilated cardiomyopathy: simultaneous Doppler echocardiography and hemodynamic evaluation. J Am Soc Echocardiogr 1991; 4: 349-60.

Stangl K, Seitz K, Wirtzfeld A, Alt E, Blomer H. Differences between atrial single chamber pacing (AAl) and ventricular single chamber pacing (NVI) with respect to prognosis and antiarrhythmic effect in patients with sick sinus syndrome. PACE-Pacing-Clin-Electrophysiol 1990; 13: 2080-5

Stauffer JC, Gaasch WH. Recognition and treatment of left ventricular diastolic dysfunction. Prog Cardiovasc Dis 1990; 32: 319- 32

Stehouwer CDA, Nauta JJP, Zeldenrust GC, Hackeng WHL, Donker AJM, den Ottolander GJH. Urinary albumin excretion, cardiovascular disease, and endothelial dysfunction in noninsulin dependent diabetes mellitus. Lancet 1992; 340: 319-23.

Stewart RAH, Joshi J, Alexander N, Nihoyannopoulos P, Oakley CM. Adjustment for the influence of age and heart rate on Doppler measurements of left ventricular filling. $\mathrm{Br}$ Heart $\mathrm{J}$ 1992; 68: 608-12.

Stoddard MF, Pearson AC, Kern MJ, Ratcliff J, Mrosek DG, Labovitz AJ. Left ventricular diastolic function: Comparision of pulsed Doppler Echocardiography and hemodynamic indexes in subjects with and without coronary artery disease. J Am Coll Cardiol 1989; 13: 327-36.

Stone MC, Thorp JM. Plasma fibrinogen - a major coronary risk factor. J Roy Coll Gen Prac 1985;35: 565-69.

Strasberg B, Sagie A, Rechavia E, Katz A, Ovsyscher IA, Sclarovsky S, Agmon J. Deleterious effects of intravenous verapamil in Wolff-Parkinson-White patients and atrial fibrillation. Cardiovasc Drugs Ther 1989; 2: 801-6.

Stratton JR, Lighty GW, Pearlman AS, Ritchie J. Detection of left ventricular thrombus by twodimensional echocardiography: sensitivity, specificity and causes of uncertainty. Circulation 1982; 66: 156-66.

Stratton JR, Nemanich JW, Johannessen KA, Resnick AD. Fate of left ventricular thrombi in patients with remote myocardial infarction or idiopathic cardiomyopathy. Circulation 1988; 78 : 1388-93.

Stratton JR, Ritchie JL 111 In platelet imaging of left ventricular thrombi: Predictive value for systemic emboli. Circulation 1990; 81: 1182-89.

Stroke Prevention in Atrial Fibrillation Investigators. Predictors of thromboembolism in atrial fibrillation: I. Clinical features of patients at risk. Ann Intern Med 1992; 116: 1-5.

Stroke Prevention in Atrial Fibrillation Investigators. Predictors of thromboembolism in atrial fibrillation: II. Echocardiographic features of patients at risk. Ann Intern Med 1992; 116: 6-12.

Stroke Prevention in Atrial Fibrillation Investigators. Stroke Prevention in Atrial Fibrillation Study. Final results. Circulation 1991;84: 527-39.

Stroke Prevention in Atrial Fibrillation Study Group Investigators. Preliminary report of the Stroke Prevention in Atrial Fibrillation Study. N Engl J Med 1990; 322: 863-8 
Suttorp MJ, Kingma JH, Jessurun ER, Lie-A-Huen L, van Hemel NM, Lie KI. The value of class IC antiarrhythmic drugs for acute conversion of paroxysmal atrial fibrillation or flutter to sinus rhythm. J Am Coll Cardiol 1990; 16: 1722-7.

Suttorp MJ, Kingma JH, Lie A Huen L, Mast EG. Intravenous flecainide versus verapamil for acute conversion of paroxysmal atrial fibrillation or flutter to sinus rhythm. Am J Cardiol 1989; 63: $693-6$

Takahashi N, Imataka K, Seki A, Fujii J. Left atrial enlargement in patients with paroxysmal atrial fibrillation. Jpn Heart J 1982: 23: 677-83.

Takahashi N, Seki A, Imataka K, Fujii J. Clinical features of paroxysmal atrial fibrillation: An observation of 94 patients. Jpn Heart J 1981; 22: 143-49.

Takemoto KA, Bernstein L, Lopez JF, Marshak D, Rahimtoola SH, Chandraratna PAN. Abnormalities of diastolic filling of the left ventricle associated with aging are less pronounced in exercise-trained individuals. Am Heart J 1992; 124: 143-48.

Teichholz LE, Kreulen T, Herman MV, Gorlin R. Problems in echocardiographic volume determinations: echocardiographic-angiographic correlations in the presence or absence of asynergy. Am J Cardiol 1976; 37: 7-11.

Thomas JD, Weyman AE. Echocardiographic Doppler evaluation of left ventricular diastolic dysfunction. Circulation 1991 84: 977-90.

Tomita M, Spinale FG, Crawford FA, Zile MR. Changes in left ventricular volume, mass and function during the development and regression of supraventricular tachycardia-induced cardiomyopathy. Disparity betwen recovery of systolic versus diastolic function. Circulation 1991; 83: 635-44.

Tranchesi B, Chamone DF, Cobbaert C, Van de Werf F, Vanhove P, Verstraete M. Coronary recanalization rate after intravenous bolus of alteplase in acute myocardial infaction. Am J Cardiol 1991; 68: 161-165.

Tranchesi B, Maranhao R, Cobbaert C, Vanhove P, Verstraete M. Lack of association between raised serum lipoprotein (a) and thrombolysis. Lancet 1990; 336: 1587-88.

Truitte DB, Olmstead EM, O'Connor GT, Plehn JF. Failure of atrial size to predict recurrence of atrial fibrillation after successful cardioversion: An echocardiographic study. J Am Coll Cardiol 1993; 21: 203A (abstract).

Tsai LM, Chen JH, Fang CJ, Lin LJ, Kwan CM. Clinical implications of left atrial spontaneous echo contrast in nonrheumatic atrial fibrillation. Am J Cardiol 1992; 70: 327-31.

Uchiyama S, Takeuchi M, Osawa M, Kobayashi I, Maruyama S, Aosaki M, Hirosawa K. Platelet function tests in thrombotic cerebrovascular disorders. Stroke 1983; 14: 511-17.

Utermann G, Kraft HG, Menzel HJ, Hopferwieser T, Seitz C. Genetics of the quantitative Lp(a) trait. I relation of $L p(a)$ glycoprotein phenotype to $L p(a)$ lipoprotein concentrations in plasma. Hum Genet 1988; 78: 41-6.

Van Gelder IC, Crijns HJ, Van der Laarse A, Van Gilst WH, Lie KI. Incidence and clinical significance of ST segment elevation after electrical cardioversion of atrial fibrillation and atrial flutter. Am Heart J 1991; 121: 51-6

Van Gelder IC, Crijns HJ, Van Gilst WH, Hamer HPM, Lie KI. Decrease of right and left atrial sizes after direct-current electrical cardioversion in chronic atrial fibrillation. Am J Cardiol 1991; 67: 93-5.

Van Gelder IC, Crijns HJ, Van Gilst WH, Verwer R, Lie KI. Prediction of uneventful cardioversion and maintainence of sinus rhythm from direct-current electrical cardioversion of chronic atrial fibrillation and flutter. Am J Cardiol 1991; 68: 41-6. 


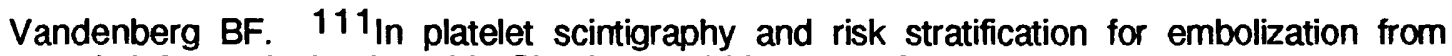
chronic left ventricular thrombi. Circulation 1990; 81: 1419-21.

Villari B, Hess OM, Kaufmann P, Krogmann ON, Grimm J, Krayenbuehl HP. Effect of aortic valve stenosis (pressure overload) and regurgitation (volume overload) on left ventricular systolic and diastolic function. Am J Cardiol 1992; 69: 927-34.

Virchow R. [Gesammelte abhandlungen zur wissenschaftlichen medtzin.] Frankfurt: Medinger Sohn \& Co, 1856: 219-732.

Visser CA, Kan G, David GK, Lie KI, Durrer D. Two dimensional echocardiography in the diagnosis of left ventricular thrombus: a prospective study of 67 patients with anatomic validation. Chest 1983; 83: 228-32.

Vitolo E, Tronci M, Larovere MT, Rumolo R, Morabito A. Amiodarone versus quinidine in the prophylaxis of atrial fibrillation. Acta Cardiol 1981; 36: 431-44.

von Hodenberg E, Kreuzer J, Hautmann M, Nordt T, Kübler W, Bode C. Effects of lipoprotein (a) on success rate of thrombolytic therapy in acute myocardial infarction. Am J Cardiol 1991; 67: 1349-53.

Wahlberg T, Blomback M, Overmark I.Blood coagulation studies in 45 patients with ischemic cerebrovascular disease and 44 patients with venous thromboembolic disease. Acta Med Scand 1980; 207: 385-90.

Warren SE, Grossman W. Therapeutic approaches affecting diastolic function. Herz 1991; 16: 33-45.

Weinberg $\mathrm{D}$, Mancini $\mathrm{J}$. Anticoagulation for cardioversion of atrial fibrillation. Am J Cardiol 1989; 63: 745-46.

Weinreich DJ, Burke JF, Pauletto FJ. Left ventricular mural thrombi complicating acute myocardial infarction: long term follow-up with serial echocardiography. Ann Intern Med 1984; 100: $789-94$.

Welin L, Svardsudd K, Wilhelmsen L, Larsson B, Tibblin G. Analysis of risk factors for stroke in a cohort of men born in 1913. N Engl J Med 1987; 317: 521-6.

Wilhelmsen L, Svardsudd K, Korsan-Bengtsen K, Larsson B, Welin L, Tibblin G. Fibrinogen as a risk factor for stroke and myocardial infarction. N Engl J Med 1984; 311:501-5.

Wolf PA, Abbot RD, Kannell WB. Atrial fibrillation as an independent risk factor for stroke: the Framingham study. Stroke 1991; 22 : 983-8.

Wolf PA, Abott RD, Kannell WB. Atrial fibrillation: a major contributor to stroke in the elderly. The Framingham Study. Arch Intern Med 1987; 147: 1561-64.

Wolf PA, Dawber TR, Thomas HE Jr, Kannel WB. Epidemiologic assessment of chronic atrial fibrillation and risk of stroke: the Framingham study. Neurology 1978; 28: 973-7.

Wolf PA, Kannel WB, McGee DL, Meeks SL, Bharucha NE, McNamara PM. Duration of atrial fibrillation and imminence of stroke: the Framingham study. Stroke 1983; 14: 664-7.

Woo J, Lau E, Lam CWK, Kay R, Teoh R, Wong HY, Prall WY, Kreel L, Nicholls MG. Hypertension, lipoprotein (a) and apolipoprotein A-I as risk factors for stroke in the Chinese. Stroke 1991; 22: 203-8.

Wooff N, Davies MJ. Interrelationship between atherosclerosis and thrombosis. In: Fuster V, Verstraete $M$ (ed) Thrombosis in cardiovascular disorders. Philadelphia. WB Saunders. 1992;41-78.

Yamamoto K, Ikeda U, Shimada K. A hypercoagulable state in the left atrium of patients with mitral stenosis. N Engl J Med 1993; 328: 1043-44. 
Yamanouchi H, Tomonaga M, Shimada H, Matsushita S, Kuramoto K, Toyokura Y. Novalvular atrial fibrillation as a cause of fatal massive cerebral infarction in the elderly. Stroke 1989; 20: 1653-56.

Yapa RSS, Green GJ. Embolic stroke following cardioversion of atrial fibrillation to sinus rhythm with oral amiodarone therapy. Postgrad Med J 1990; 66: 410.

Yarnell JW, Baker IA, Sweetnam PM, Bainton D, O'Brien JR, Whitehead PJ, Elwood PC. Fibrinogen, viscosity, and white blood cell count are major risk factors for ischemic heart disease. The Caerphilly and Speedwell Collaborative Heart Disease Studies. Circulation 1991; 83: 836-44.

Yasaka M, Miyatake K, Mitani M, Beppu S, Nagata S, Yamaguchi T, Omae T. Intracardiac mobile thrombus and D-dimer fragment of fibrin in patients with mitral stenosis. Br Heart J 1991; 66: 22-5.

Zanini R, Facchinetti AI,Gallo G, Cazzamalli L, Bonandi L, Dei Cas L. Morbidity and mortality of patients with sinus node disease: comparative effects of atrial and ventricular pacing. PACEPacing-Clin-Electrophysiol 1990; 13: 2076-9

Zannad F, Stoltz JF. Blood rheology in arterial hypertension. J Hypertension 1992; 10 (suppl): S69-78.

Zenker G, Költringer P, Boné G, Niederkorn K, Pfeiffer K, Jürgens G. Lipoprotein (a) as a strong indicator for cerebrovascular disease. Stroke 1986; 17: 942-45.

Zipes DP. Management of cardiac arrhythmias: Pharmacological, Electrical and Surgical techniques. In: Braunwald $\mathrm{E}$ (ed) Heart Disease. A textbook of cardiovascular medicine. Philadelphia: WB Saunders 4th ed. $1992: 628-66$.

Zipes DP. Specific arrhythmias: Diagnosis and Treatment. In: Braunwald E (ed) Heart Disease. A Textbook of Cardiovascular Medicine. Philadelphia: 4th ed. WB Saunders. 1992; 667-725. 